Universidade de São Paulo

Instituto de Física

\title{
Fotofísica e propriedades dinâmicas de sistemas moleculares
}

\author{
Yoelvis Orozco González
}

Tese de doutorado apresentada ao Instituto de Física para a obtenção do título de Doutor em Ciências

Orientador: Prof. Dr. Sylvio Roberto Accioly Canuto

\section{Banca examinadora:}

Prof. Dr. Sylvio Roberto Accioly Canuto (IF/USP)

Prof. Dr. Sebastian Fernandez Alberti (Universidad Nacional de Quilmes, Argentina)

Prof. Dr. Antonio Carlos Borin (IQ/USP)

Prof. Dr. Marcio Teixeira do Nascimento Varella (IF/USP)

Profa. Dra. Lucy Vitoria Credidio Assali (IF/USP) 


\section{FICHACATALOGRÁFICA \\ PreparadapeloServiçodeBibliotecaelnformação dolnstitutodeFísicadaUniversidadedeSãoPaulo}

Gonzáles, YoelvisOrozco

Fotofísica e propriedades dinâmicas de sistemas moleculares. -SãoPaulo,2012.

Tese(Doutorado) - UniversidadedeSãoPaulo.

Instituto de Física - Depto. de Física Geral

Orientador:Prof.Dr.Sylvio Roberto Accioly Canuto

ÁreadeConcentração:Física

Unitermos: 1.Estrutura Eletrônica; 2. Fotofísica;

3. Dinâmica Molecular; 4. Soluções - Efeitos do Solvente; 5. Estados Excitados. 
Amicsposa o hija 



\section{Sumário}

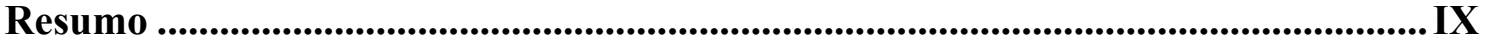

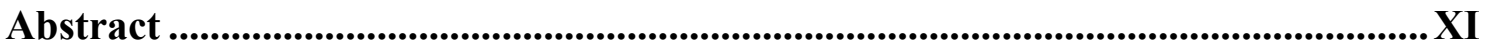

Lista de Abreviaturas....................................................................................................

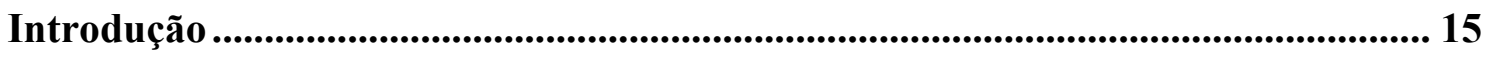

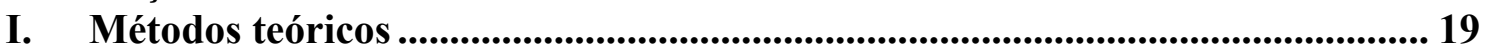

1. Teoria dos cálculos de mecânica quântica..................................................................... 21

1.1. A aproximação de Born-Oppenheimer...................................................... 22

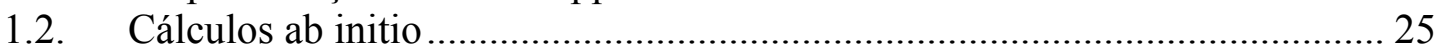

1.3. A aproximação de Hartree-Fock................................................................. 26

1.4. Cálculos além de Hartree-Fock ..................................................................... 29

1.4.1. Métodos de interação de configurações e campo autoconsistente

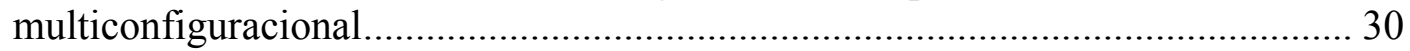

1.4.2. Métodos perturbativos (Rayleigh-Schrödinger) ................................... 32

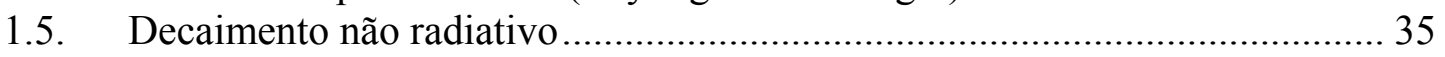

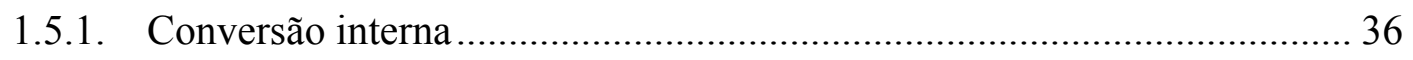

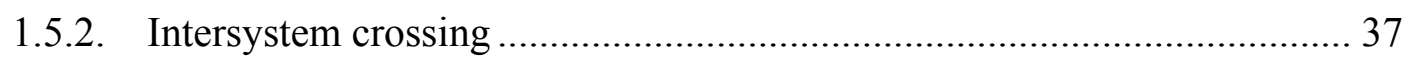

2. Superfícies de energia potencial e método de trajetórias quase-clássicas ....... 41

3.1. Superfície de energia potencial DMBE ...................................................... 41

3.2. Modelo quase-clássico para reações bi-moleculares ................................... 44

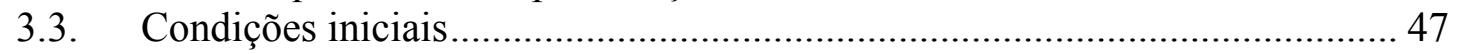

3. Simulação clássica de Monte Carlo...............................................................53

3.1. Processo Markoviano e amostragem de Metropolis ...................................... 54

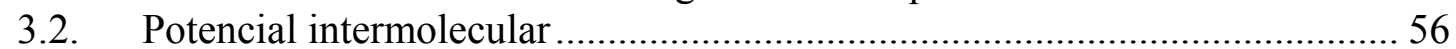

3.3. Função de autocorrelação da energia..................................................... 57

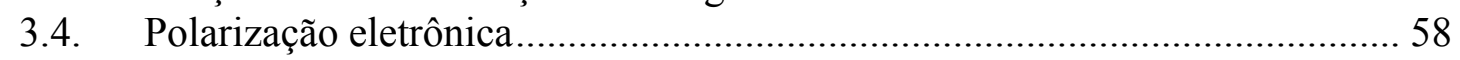

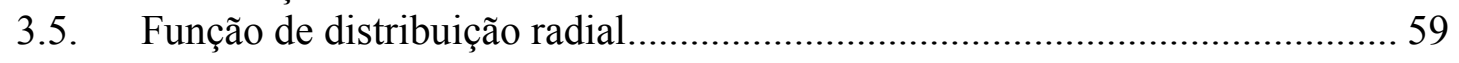

II. Sistemas estudados .................................................................................. 61

4. Estudo de trajetórias quase-clássicas da reação $\mathrm{OH}+\mathrm{SO}$ : Influência da energia

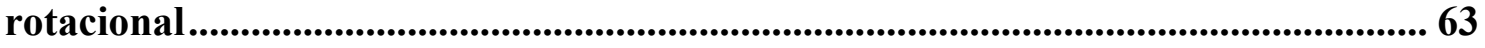

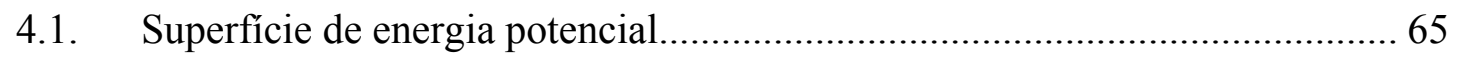

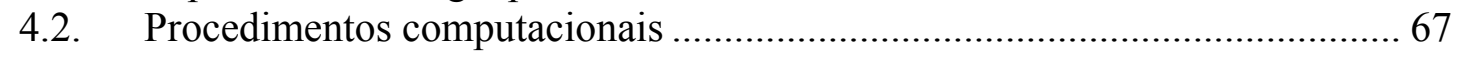

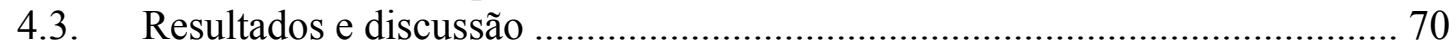

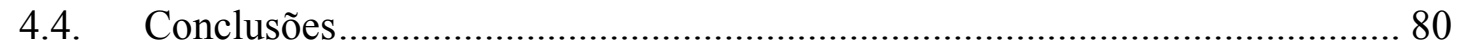


5. Estudo da superfície de energia potencial do sistema molecular $\mathrm{HSO}_{2}$ no nível

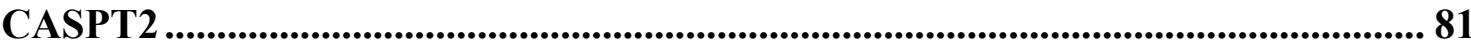

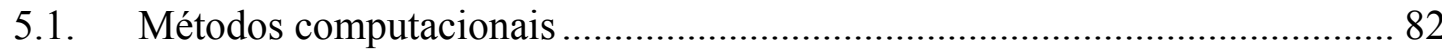

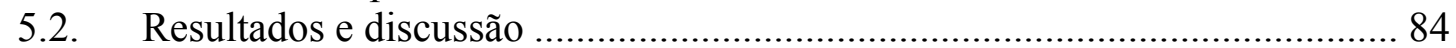

5.2.1. Caminho de reação $\mathrm{SH}+\mathrm{O}_{2} \rightarrow \mathrm{HSO}+\mathrm{O}$................................................... 86

5.2.2. Caminhos de reação $\mathrm{SH}+\mathrm{O}_{2} \rightarrow \mathrm{OH}+\mathrm{SO}$ (região de baixas energias),

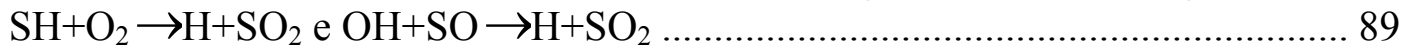

5.2.3. Caminhos de reação $\mathrm{SH}+\mathrm{O}_{2} \rightarrow \mathrm{OH}+\mathrm{SO}$ (região de maiores energias)...... 92

5.2.4. Características gerais dos caminhos de reação mostrados nos diagramas. 94

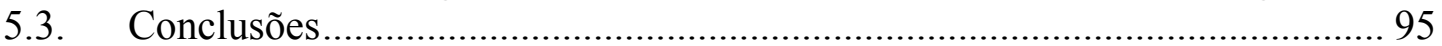

6. Polarização do estado excitado e reavaliação da emissão $n \leftarrow \pi *$ da acetona em

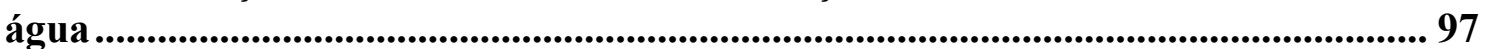

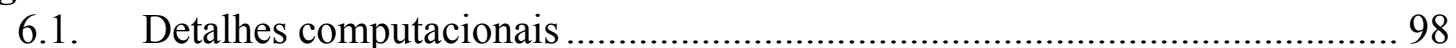

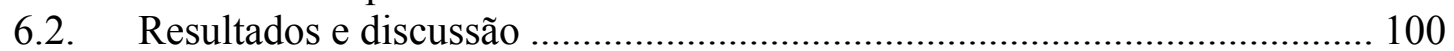

6.2.1. Polarização do soluto no estado eletronico fundamental e primeiro estado

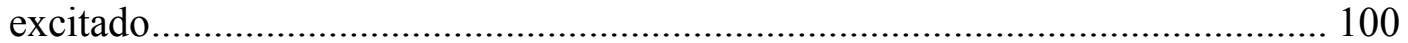

6.2.2. Efeito de solvente na emissão $n \leftarrow \pi^{*}$.................................................. 103

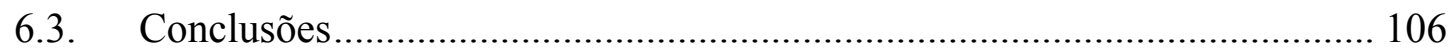

7. Estudo teórico da fotofísica do sistema molecular 1-nitronaftaleno e efeito dos solventes metanol e etanol ........................................................................................... 109

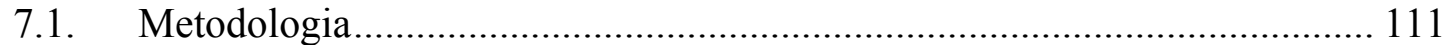

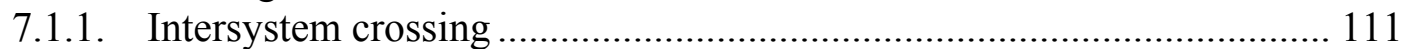

7.1.2. Transições eletrônicas e efeito de solvente............................................... 113

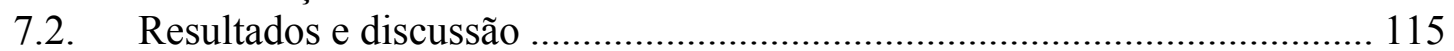

7.2.1. Mecanismo de desativação proposto ..................................................... 115

7.2.2. Espectros de absorção transitória incluindo o efeito de solventes........... 119

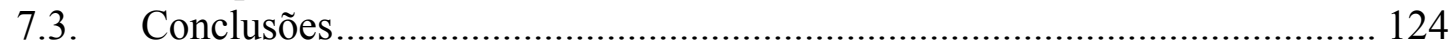

8. Efeito do solvente no Stokes shift e no decaimento não fluorescente do sistema

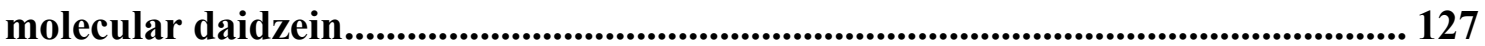

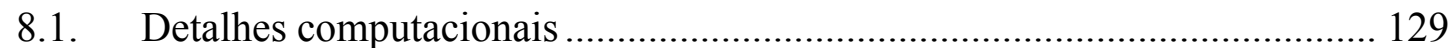

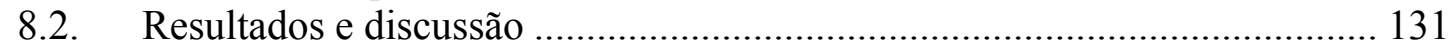

8.2.1. Espectro de absorção ......................................................................... 131

8.2.2. Espectro de fluorescência em água....................................................... 134

8.2.3. Decaimento não fluorescente em acetonitrila........................................ 136

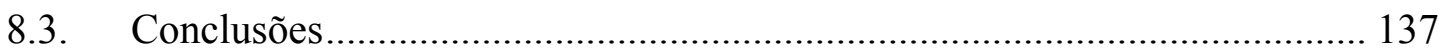

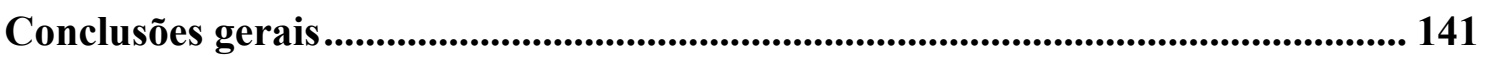

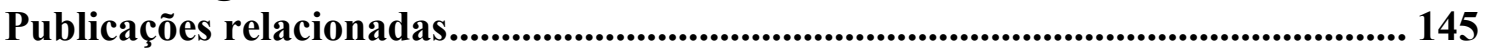

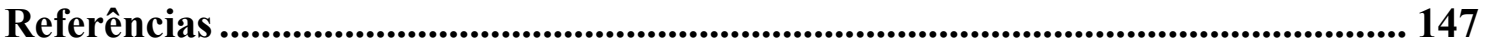




\section{Agradecimentos}

A realização desta tese de doutoramento representa uma etapa bastante significativa da minha vida. Tive a oportunidade de conhecer o maravilhoso povo brasileiro e a sua paixão pelo futebol e pelo samba. Conheci pessoas maravilhosas que me ajudaram muito todo esse tempo e que graças a elas foi muito mais fácil minha vida longe de casa. Academicamente, foi uma etapa de muitos desafios e muito trabalho, mas, foi gratificante demais aprender de pessoas competentes e de formar parte de um grupo de trabalho tão excepcionalmente unido e profissional.

Dito tudo isso, desejo expressar explicitamente alguns agradecimentos.

- Ao CNPq pelo apoio financeiro e ao Instituto de Física pelas excelentes condições de trabalho que me proporcionou.

- Aos amigos que têm estado no meu lado todo esse tempo, pela agradável convivência e a colaboração científicas, em particular ao meu velho amigo Israel, a Rodrigo, Rafael, Lucas, Marcelo, Daniel, Paula Jaramillo, Bistafa, Fernando, João e Ednilson. Agradeço muito a Rodrigo e Rafael pela ajuda e o apoio que me ofereceram logo no começo da minha chegada ao Brasil.

- A minha esposa Dayana, não sei como lhe agradecer tanta ajuda, apoio, compreensão e sacrifício. Você secou meus olhos quando estive triste, me levantou quando estive fraco e desfrutou comigo cada alegria. Você é meu anjo!

- A mi querida madre y a mi maravilloso padre por haber cuidado de mi hija Rosianet y por haberle dado tanto amor; el mismo amor que yo recibí toda mi vida y por el cual los he extrañado tanto todo este tiempo. A mi abuela Caridad, a mi abuelo Juan y a mis tíos Luis y Juan Roberto les agradezco mucho por el cariño. A mis suegros Alina y Overlan también les agradezco por haber cuidado de mi niña y por ser unos suegros ejemplares.

- Aos professores Maikel Ballester y Juan de Dios Garrido pela orientação acadêmica desde o começo da minha formação profisssional e pela amizade.

- À professora Kaline Coutinho pela ajuda nas simulações.

- Finalmente desejo agradecer ao meu orientador, o professor Sylvio Canuto. Eu lhe agradeço pela oportunidade, pela confiança, pela amizade e pela excelente orientação profissional. Sinto um grande orgulho de ter sido seu orientando. 


\section{Resumo}

A fotodinâmica de sistemas moleculares representa um dos principais tópicos atuais da físico-química molecular. O conhecimento das propriedades dos estados eletrônicos excitados tem permitido desenvolver áreas de vital importância como das energias renováveis, da fotomedicina, dos sensores fluorescentes, entre outras. O objetivo desta tese está orientado a estudar teoricamente a influência do meio (ou efeito de solvente) na fotofísica e nas propriedades dos estados eletrônicos excitados de sistemas moleculares.

Nesta tese, primeiramente foi feito um estudo em fase gasosa da superfície de energia potencial do sistema molecular $\mathrm{HSO}_{2}$ e do efeito da energia rotacional na reação $\mathrm{OH}+\mathrm{SO}$. Na superfície de energia potencial foram caracterizadas um grande número de estruturas estacionárias e foi encontrado um estado de transição que liga a região mais energética da superfície com a menos energética. Em relação ao papel da energia rotacional na reação mencionada, foi realizado um estudo de trajetórias quase-clássicas, onde foi observado um decréscimo da reatividade com o aumento da energia rotacional total depositada nos reagentes.

Posteriormente, foi estudado o efeito do solvente nas propriedades dos estados eletrônicos excitados e nos mecanismos de decaimento de três sistemas moleculares, acetona, 1-nitronaftaleno e daidzein. $\mathrm{Na}$ acetona, foi estudada a influência da polarização eletrônica do estado excitado $n \rightarrow \pi^{*}$ provocada pelo solvente no deslocamento espectral da banda de fluorescência. A banda de emissão obtida em água 
mostra um deslocamento espectral muito pequeno em relação à fase gasosa, em concordância com as evidencias experimentais. Também foi observada pouca dependência do deslocamento espectral com o grau de polarização eletrônica desse estado excitado. $\mathrm{O}$ sistema molecular 1-nitronaftaleno foi estudado a fim de esclarecer a ultrarápida desativação eletrônica não fluorescente observada experimentalmente após a transição de absorção, assim como, caracterizar os espectros de absorção transitória também observados nos experimentos. Foi encontrado um intersystem crossing muito eficiente entre o primeiro estado excitado singleto e o segundo estado tripleto, que explica o decaimento não fluorescente deste sistema molecular. O modelo de decaimento proposto permite descrever corretamente os espectros de absorção transitória nos solventes metanol e etanol, através de transições de absorção dos estados eletrônicos tripletos. Finalmente, o sistema molecular daidzein foi estudado a fim de entender porque em solvente polar prótico, como a água, o sistema é fluorescente, mostrando um valor de Stokes shift consideravelmente grande e na presença de solvente polar aprótico, como a acetonitrila, não é observada fluorescência. Nesse sentido, foi estudada a evolução dos estados eletrônicos excitados, na presença dos solventes água e acetonitrila, após as transição de absorção. A topologia dos estados eletrônicos excitados é diferente para cada um dos solventes, em acetonitrila o sistema tem acesso a um intersystem crossing muito eficiente que permite o decaimento não fluorescente. Em água o panorama é diferente, neste caso, não é possível a ocorrência do intersystem crossing e o sistema decai por fluorescência para o estado fundamental. No estado eletrônico fluorescente é observada uma polarização eletrônica significativa que provoca o grande valor de Stokes shift observado experimentalmente. 


\section{Abstract}

The photodynamics of molecular systems represents one of the most important topics of the molecular physical chemistry today. The knowledge of the excited electronic states properties has allowed the development of several important areas, such as the renewable energies, the photomedicine, fluorescent sensors, etc. The aim of this thesis is oriented to the theoretical study of the solvent effect on the photophysics and on the excited electronic states properties of molecular systems.

In this thesis, it was initially studied the potential energy surface of the $\mathrm{HSO}_{2}$ molecular system in gas phase and the rotational energy effect on the reactivity of the $\mathrm{OH}+\mathrm{SO}$ reaction. In the potential energy surface a large number of stationary structures were characterized and it was found a transition state which connects the highest energetic region to the lowest one. Regarding the role of rotational energy on the mentioned reaction, a quasi-classical trajectories study was performed, indicating a decrease in the reactivity when the total rotational energy deposited in the reactants is increased.

Subsequently, it was studied the solvent effect on the excited electronic states and on the deactivation mechanisms of three molecular systems, acetone, 1-nitronaphthalene and daidzein. In the acetone molecular system, it was studied the influence of the electronic polarization, caused by the solvent, in the fluorescence spectral shift of the $n \rightarrow \pi^{*}$ excited state. The emission band obtained in water shows a small spectral shift compared to the gas phase, in agreement with the experimental evidences. It was also 
observed a little dependence of the spectral shift with the degree of the excited state polarization. The 1-nitronaphthalene molecular system was studied to clarify the ultrafast non-fluorescent deactivation mechanism experimentally observed after the absorption transitions, as well as to characterize the transient absorption spectra also observed in the experiments. A very efficient intersystem crossing was found between the first singlet excited state and the second triplet state, which explains the nonfluorescent decay of this molecular system. The proposed deactivation model allows properly describing the transient absorption spectra in methanol and ethanol solvents by absorption transitions from the triplet electronic states. Finally, the daidzein molecular system was studied to understand why in polar protic solvent, such as water, the system is fluorescent, showing a very large Stokes shift value and in polar aprotic solvent, such as acetonitrila, the fluorescence is not observed. In that sense, it was studied the evolution of the excited electronic states in water and in acetonitrile after the absorption transition. The topology of the excited electronic states is different for each solvent, in acetonitrile the system is accessible to a very efficient intersystem crossing that enables the non-fluorescent decay. In water the picture is different, the intersystem crossing is not possible to occur and the system decays by fluorescence to the ground electronic state. In the fluorescent state is observed a considerable electronic polarization that causes the so large Stokes shift value experimentally observed. 


\section{Lista de Abreviaturas}

ANO-L

ASEC

AVDZ

AVTZ

B3LYP

CASPT2

CASSCF

$\operatorname{CCSD}(\mathrm{T})$

CHELPG

CI

CIS

CIS(D)

CISD

CISDT

CISDTQ

CNDO-CI

CPMD

DFT

DMBE

EFGS

EFS

FCI

FVCAS

FWHM
Atomic Natural Orbitals with Large primitive set

Average Solvent Eletrostatic Configuration

Conjunto de funções-base aug-cc-pVDZ

Conjunto de funções-base aug-cc-pVTZ

Método DFT com funcional híbrido de exchange de Becke e funcional de correlação de Lee-Yang-Parr

Complete Active Space Second-order Perturbation Theory

Complete Active Space Self-Consistent Field

Coupled-Cluster with Single and Double and perturbative Triple excitations

Charges from Electrostatic Potentials using a Grid based method

Conversão Interna

Configuration Interaction with Single excitations

Configuration Interaction with Single and perturbative Double excitations

Configuration Interaction with Single and Double excitations

Configuration Interaction with Single, Double and Triple excitations

Configuration Interaction with Single, Double, Triple and Quadruple excitations

Complete Neglect of Differential Overlap with Configuration Interaction

Car-Parrinello Molecular Dynamics

Density Functional Theory

Double Many-Body Expansion

Electric Feld and Gradient Set

Electric Field Set

Full Configuration Interaction

Full Valence Complete Active Space

Full Width at Half Maximum 
HF

Hartree-Fock

HOMO

Highest Occupied Molecular Orbital

INDO

Intermediate Neglect of Differential Overlap

ISC

Intersystem Crossing

LJ

Lennard-Jones

LUMO

Lowest Unoccupied Molecular Orbital

MBE

Many-Body Expansion

$\mathrm{MC}$

Monte Carlo

$\mathrm{MCSCF}$

Multi-Configurational Self-Consistent Field

MDDF

Minimum Distance Distribution Function

MEP

Minimum Energy Path

MP

Møller Plesset

MP2

MP4

Møller Plesset Second order perturbation theory

MRCI

Møller Plesset Fourth order perturbation theory

NPT

Multi-Reference Configuration Interaction

Ensemble onde o número de partículas $(\mathrm{N})$, a pressão $(\mathrm{P})$ e a temperatura $(\mathrm{T})$ são fixos.

OPLS Optimized Parameters for Liquid Simulations

PCM

Polarizable Continuum Model

QCT

Quasi-Classical Trajectory

$\mathrm{QM} / \mathrm{MM}$

Quantum Mechanics/Molecular Mechanics

QMSTAT

A combined Quantum Mechanical Statistical mechanical model

RASSCF

Restricted Active Space Self Consistent Field

RASSI

$\mathrm{RDF}$

Restricted Active Space State Interaction

ROKS

Radial Distribution Function

$\mathrm{SCF}$

Restricted Open-shell Kohn-Sham

SCRF

Self-Consistent Field

SEP

SOC

TD-PBE0

Self-Consistent Reaction Field

Superfície de Energia Potencial

Spin-Orbit Coupling

TIP3P

Método DFT com funcional híbrido de Perdew-Burke-Ernzerhof

TS

Three-Site Transferrable Intermolecular Potential

VEQMT

ZPE

Transition State

Vibrational Energy Quantum Mechanical Threshold

Zero Point Energy 


\section{Introdução}

A evolução dos sistemas moleculares após a absorção de luz é um tópico atual da físicoquímica molecular. $\mathrm{O}$ estudo das propriedades dos estados eletrônicos excitados tem permitido o desenvolvimento de sistemas moleculares funcionais, capazes de desencadear diferentes tipos de processos após a absorção de energia. Vários exemplos de grande relevância podem ser mencionados. No caso das células solares sensibilizadas (light harvest) ${ }^{[1]}$ é possível a conversão de energia luminosa em energia elétrica de uma forma muito eficiente e barata. A terapia fotodinâmica ${ }^{[2]}$ é outro exemplo, onde o tratamento contra varias doenças e tipos de câncer, é baseada na evolução de sistemas moleculares excitados. Por outra parte, o desenvolvimento dos sensores fluorescentes ${ }^{[3,}$ 4], em conjunto com a evolução da single-molecule spectroscopy ${ }^{[5]}$, tem permitido desenvolver métodos de processamento de imagens muito precisas, métodos de rastreamento de estruturas moleculares, de detecção de reações químicas e outros muitos processos ${ }^{[4,6]}$.

Toda essa aplicabilidade, decorrente das propriedades dos estados eletrônicos excitados de sistemas moleculares, carrega grandes desafios para os métodos teóricos, principalmente sabendo que todos esses processos geralmente ocorrem interagindo com um ambiente líquido. A influência do meio nas propriedades moleculares é um elemento muito importante que deve ser levado em consideração nos estudos teóricos, pois inevitavelmente tem uma influência relevante na atividade dos sistemas moleculares. Essa não é uma tarefa fácil, pois o grande número de átomos envolvidos, 
mesmo nos sistemas mais simples, faz com que as equações da mecânica quântica não possam ser aplicadas com toda a formalidade.

A presente tese está orientada a estudar como são modificadas as propriedades dos estados eletrônicos excitados de sistemas moleculares ao interagir com o meio, desde que a grande maioria de processos químicos e biológicos acontecem em meio líquido.

Os métodos tradicionais que consideram os efeitos do solvente através das teorias clássicas de Onsager e Kirkwood ${ }^{[7,8]}$, da Teoria de Campo de Reação Auto-consistente $(\mathrm{SCRF})^{[9,10]}$ ou do modelo continuo polarizável $(\mathrm{PCM})^{[11]}$, são métodos que têm mostrado muitas debilidades. Estas debilidades devem-se, entre outros fatores, a que não são consideradas explicitamente as moléculas do meio e não é possível considerar interações importantes entre sítios moleculares como, por exemplo, as ligações de hidrogênio. Tudo isso, carrega a necessidade de criar outros modelos teóricos onde as moléculas dos líquidos (solventes) possam ser consideradas explicitamente. A teoria do funcional da densidade (DFT) tem ajudado muito neste sentido, permitindo tratar quanticamente a dinâmica de sistemas moleculares, no estado eletrônico fundamental, que podem ser formados por até várias centenas de átomos. Dessa forma, vários métodos têm sido desenvolvidos, como a dinâmica de Carr-Parrinello (CPMD) ${ }^{[12]}$ e a dinâmica de Born-Oppenheimer ${ }^{[13]}$, entre outras.

O problema no estudo dos estados eletrônicos excitados é um pouco mais complicado, por varias razões. Primeiramente, as funções de onda de estados eletrônicos excitados são principalmente determinadas usando métodos multiconfiguracionais ou interação de configurações, limitando assim as dimensões do sistema a ser tratado quanticamente. Em segundo lugar, existe uma grande variedade de processos físicoquímicos desencadeados pela excitação dos sistemas moleculares, como transferências de carga, transferência de energia, fotodissociação, isomerização, fluorescência, fosforescência, decaimento não radiativo, etc., que representam tópicos atuais da físicoquímica teórica e representam um desafio ainda maior quando é preciso considerar a interação com o meio.

As metodologias mais usadas no estudo destes sistemas moleculares em meio líquido combinam cálculos de mecânica quântica com cálculos de mecânica molecular (QM/MM). Nestes casos, a região molecular de maior interesse é tratada com o hamiltoniano quântico, enquanto o resto do sistema é considerado classicamente. Na forma tradicional desta metodologia, a cada passo da simulação clássica é feito um cálculo de mecânica quântica no subsistema descrito pelo hamiltoniano quântico, 
tirando informações dos gradientes, energias, probabilidade de transição, etc. Enquanto, outra variante da metodologia QM/MM, foi proposta por Coutinho e Canuto ${ }^{[14]}$, chamada de QM/MM-sequencial. Neste caso, é realizada a simulação clássica do líquido a fim de gerar configurações representativas do ensemble estudado e que posteriormente são usadas nos cálculos de mecânica quântica. Esta metodologia QM/MM-sequencial, é muito viável do ponto de vista computacional, mas, não oferece informação temporal do sistema, desde que é baseada no método de Monte Carlo. Daí aparecem algumas dificuldades para trabalhar nos estados eletrônicos excitados, onde o equilíbrio eletrostático com o solvente depende dos tempos de vida dos estados excitados. No entanto, algumas aproximações podem ser feitas a fim de ter um relativo controle do equilíbrio eletrostático com o solvente e da polarização eletrônica dos estados eletrônicos excitados e que serão aplicadas em alguns dos trabalhos apresentados nesta tese.

$\mathrm{Na}$ presente tese são apresentados dois trabalhos teóricos relacionados ao sistema molecular $\mathrm{HSO}_{2}$ em fase gasosa. A idéia inicial do estudo deste sistema molecular era considerar diferentes fatores, como efeito do meio e excitações ro-vibracionais, que influenciam na reatividade de reações químicas contempladas na superfície de energia potencial deste sistema e que apresentam interesse atmosférico. A influência das excitações vibracionais dos reagentes em algumas destas reações já foram estudadas em trabalhos anteriores. Portanto, no primeiro trabalho apresentado nesta tese é estudado o efeito da energia rotacional dos reagentes na reatividade da reação $\mathrm{OH}+\mathrm{SO}$. A solvatação, ou microsolvatação de caminhos de reação, seria também estudada para analisar a influência da água da troposfera nas reações químicas. Mas, este estudo não foi realizado, pois ficou em dúvida o mecanismo de conexão entre as regiões mais energética e menos energética da superfície de energia potencial do $\mathrm{HSO}_{2}$ em fase gasosa, devido à publicação de um trabalho teórico ${ }^{[15]}$ que discordava com o mecanismo já proposto. Assim, o estudo do sistema molecular $\mathrm{HSO}_{2}$ foi reorientado à reavaliação de algumas regiões da superfície de energia potencial em fase gasosa. Este é o segundo trabalho apresentado nesta tese, onde trata-se de caracterizar as estruturas estacionárias deste sistema molecular, incluindo um estado de transição que ligue as regiões de maiores e menores energias da superfície de energia potencial.

$\mathrm{Na}$ tese são apresentados também três trabalhos teóricos relacionados com o efeito de solvente em estados excitados. Estes trabalhos foram realizados a fim de estender as experiências acumuladas por nosso grupo de trabalho na solvatação de sistemas 
moleculares ao estudo de estados eletrônicos excitados. Um dos trabalhos está relacionado com os deslocamentos espectrais da emissão da acetona em água, tentando esclarecer a influência da polarização eletrônica do estado excitado no espectro de emissão. Posteriormente é apresentado um estudo teórico relacionado à fotofísica do sistema molecular 1-nitronaftaleno. Neste sistema trata-se de explicar a ultrarápida troca de multiplicidade observada experimentalmente neste sistema molecular após a transição de absorção. Os espectros de absorção transitória observados neste sistema molecular também são estudados incluindo o efeito do solvente. Finalmente é estudada a fotofísica do sistema molecular daidzein, da família dos flavonóides, a fim de esclarecer as causas que provocam o grande valor de Stokes shift observado experimentalmente em água e a não fluorescência em acetonitrila.

A tese está dividida principalmente em duas partes, a primeira onde são apresentados os métodos teóricos que fundamentam os estudos realizados e a segunda parte onde se discutem os sistemas estudados. 


\section{Métodos teóricos}




\section{Capítulo 1}

\section{Teoria dos cálculos de mecânica}

\section{quântica}

Neste capítulo são apresentados alguns dos métodos teóricos da mecânica quântica usados para determinar propriedades da estrutura eletrônica de sistemas moleculares. Estes métodos, em essência, tratam de resolver aproximadamente a equação de Schrödinger em sistemas moleculares.

Primeiramente será discutida a aproximação de Born-Oppenheimer [16, 17], pois representa um conceito fundamental no qual é baseado a grande maioria dos métodos de cálculos de mecânica quântica para sistemas moleculares. Em seguida será analisada a aproximação de Hartree-Fock, que é de grande importância na resolução do problema eletrônico ${ }^{[18]}$. Mais adiante será feita uma revisão dos métodos de cálculo de mecânica quântica além de Hartree-Fock, como interação de configuração, cálculos multiconfiguracionais e métodos perturbativos. 


\subsection{A aproximação de Born-Oppenheimer}

As funções de onda e as energias dos sistemas atômicos e moleculares são obtidas a partir da equação de Schrödinger, que na forma não relativista independente do tempo tem a seguinte forma:

$$
\hat{H} \psi(\mathbf{r}, \mathbf{R})=E \psi(\mathbf{r}, \mathbf{R}),
$$

onde $\mathbf{r}$ e $\mathbf{R}$ representam as coordenadas eletrônicas e nucleares respectivamente. Supondo os núcleos e elétrons como partículas pontuais e não considerando a interação spin-órbita e outras interações relativísticas, pode-se escrever o hamiltoniano molecular na seguinte forma:

$$
\hat{H}=\underbrace{-\frac{\hbar^{2}}{2} \sum_{\alpha} \frac{1}{m_{\alpha}} \nabla_{\alpha}^{2}}_{\hat{T}_{\mathrm{N}}}-\underbrace{-\frac{\hbar^{2}}{2 m_{e}} \sum_{i} \nabla_{i}^{2}}_{\hat{T}_{\mathrm{e}}}+\underbrace{\sum_{\alpha} \sum_{\beta>\alpha} \frac{Z_{\alpha} Z_{\beta} e^{2}}{R_{\alpha \beta}}}_{\hat{V}_{\mathrm{NN}}} \underbrace{\sum_{\alpha} \sum_{i} \frac{Z_{\alpha} e^{2}}{\left|\boldsymbol{R}_{\alpha}-\boldsymbol{r}_{i}\right|}}_{\hat{V}_{\mathrm{eN}}} \underbrace{\sum_{i} \sum_{i>j} \frac{e^{2}}{r_{i j}}}_{\hat{V}_{\mathrm{ee}}},
$$

onde os núcleos são representados por $\alpha$ e $\beta$ e os elétrons por $i$ e $j . R_{\alpha \beta}=\left|\mathbf{R}_{\alpha}-\mathbf{R}_{\beta}\right|$ e $r_{i j}=\left|\mathbf{r}_{i}-\mathbf{r}_{j}\right|$ representam as distâncias entre cada par núcleo-núcleo e elétron-elétron respectivamente. As magnitudes $m_{\alpha}$ e $m_{e}$ são, na ordem, as massas do $\alpha$-ésimo núcleo atômico e a massa do elétron. Daqui para frente, neste capítulo serão utilizadas as unidades atômicas $\left(e=\hbar=m_{e}=1\right)$. O primeiro termo $\left(\hat{T}_{\mathrm{N}}\right)$ da equação (1.2) é o operador de energia cinética dos núcleos atômicos e o segundo termo $\left(\hat{T}_{e}\right)$ é o operador da energia cinética dos elétrons. O terceiro termo $\left(\hat{V}_{\mathrm{NN}}\right)$ representa a energia potencial repulsiva entre os núcleos com números atômicos $Z_{\alpha}$ e $Z_{\beta}$, enquanto o quarto termo $\left(\hat{V}_{\text {eN }}\right)$ é a energia potencial atrativa entre elétrons e núcleos. O último termo $\left(\hat{V}_{\text {ee }}\right)$ indica a energia potencial das interações repulsivas entre os elétrons.

O hamiltoniano (1.2) torna extremamente complicada a resolução da equação de Schrödinger, mas fazendo algumas aproximações a resolução do problema pode-se simplificar muito. O fato de que as massas dos núcleos sejam muito maiores que as massas dos elétrons, indica que os elétrons vão se mover muito mais rápido que os núcleos atômicos, podendo-se considerar que os núcleos atômicos ficam fixos enquanto 
os elétrons se movimentam. Nessa aproximação, a magnitude $R_{\alpha \beta}$, na equação (1.2), não é mais uma variável e o termo $\hat{V}_{\mathrm{NN}}$ vira uma constante na configuração nuclear dada. Portanto, o movimento dos elétrons estará governado pela equação

$$
\hat{H}_{\mathrm{e}} \Phi_{n}(\mathbf{r} ; \mathbf{R})=\varepsilon_{n}(\mathbf{R}) \Phi_{n}(\mathbf{r} ; \mathbf{R})
$$

onde $\hat{H}_{e}$ representa o hamiltoniano puramente eletrônico, definido pela expressão

$$
\hat{H}_{\mathrm{e}}=\hat{T}_{\mathrm{e}}+\hat{V}_{\mathrm{ee}}+\hat{V}_{\mathrm{eN}}+\hat{V}_{\mathrm{NN}}
$$

$\Phi_{n}(\mathbf{r} ; \mathbf{R})$ e $\varepsilon_{n}(\mathbf{R})$ são as autofunções e autovalores do hamiltoniano eletrônico (1.4) e dependem parametricamente de R. Essas autofunções adiabáticas formam uma base completa, portanto, a função de onda molecular pode-se representar como uma combinação linear desse conjunto completo de bases:

$$
\psi(\boldsymbol{r}, \boldsymbol{R})=\sum_{n} \chi_{n}(\boldsymbol{R}) \Phi_{n}(\boldsymbol{r} ; \boldsymbol{R})
$$

Nesta expressão $\chi_{n}(\mathbf{R})$ é a função de onda nuclear na representação adiabática. Substituindo (1.5) em (1.1) e integrando por todo o espaço sobre as coordenadas eletrônicas, obtém-se um sistema de equações acopladas de seguinte forma:

$$
\left[\hat{T}(\mathbf{R})+\hat{V}_{\mathrm{NN}}(\mathbf{R})+\varepsilon_{m}(\mathbf{R})\right] \chi_{m}(\mathbf{R})+\sum_{n} \hat{\Lambda}_{m n}(\mathbf{R}) \chi_{n}(\mathbf{R})=E \chi_{m}(\mathbf{R})
$$

sendo $\hat{\Lambda}_{m n}$ os elementos matriciais do operador de acoplamento $\hat{\Lambda}$, expressados como:

$$
\hat{\Lambda}_{m n}=-\sum_{\alpha} \frac{1}{m_{\alpha}}\left(A_{m n}^{(\alpha)} \frac{\partial}{\partial R_{i}}+\frac{1}{2} B_{m n}^{(\alpha)}\right)
$$

os elementos das matrizes $\hat{\mathbf{A}}^{(\alpha)}$ e $\hat{\mathbf{B}}^{(\alpha)}$ são os seguintes:

$$
A_{m n}^{(\alpha)}=\int \Phi_{m}^{*} \frac{\partial}{\partial R_{\alpha}} \Phi_{n} \mathrm{~d}^{3} \boldsymbol{r}, \quad B_{m n}^{(\alpha)}=\int \Phi_{m}^{*} \frac{\partial^{2}}{\partial R_{\alpha}^{2}} \Phi_{n} \mathrm{~d}^{3} \boldsymbol{r}
$$

O sistema de equações acopladas (1.6) é muito complicado de resolver, mas o problema se simplifica desprezando os elementos não diagonais $\hat{\Lambda}_{m n}(m \neq n)$ da matriz 
de acoplamento $\hat{\Lambda}$. Essa aproximação é chamada de aproximação adiabática e está pressupondo que não vai haver interação entre os estados eletrônicos do sistema molecular estudado. Portanto, dentro dessa aproximação, a equação (1.5) adota a forma:

$$
\psi(\mathbf{r}, \mathbf{R})=\chi_{n}(\mathbf{R}) \Phi_{n}(\mathbf{r} ; \mathbf{R})
$$

sendo possível desacoplar as equações (1.6), e a aproximação adiabática para a função de onda nuclear obtém-se como:

$$
\hat{H}_{\mathrm{n}}^{\mathrm{ad}} \chi_{n}(\mathbf{R})=E \chi_{n}(\mathbf{R})
$$

onde o hamiltoniano adiabático nuclear é:

$$
\hat{H}_{n}^{\mathrm{ad}}=\hat{T}_{\mathrm{N}}+\varepsilon_{n}(\mathbf{R})+\hat{\Lambda}_{n n}(\mathbf{R}) .
$$

Em algumas circunstâncias, entretanto, a aproximação (1.9) não é válida e não é possível separar os movimentos eletrônico e nuclear. Geralmente, isso acontece em regiões onde os estados eletrônicos ficam muito próximos. Nestas regiões de degenerescência (ou intersecções cônicas ${ }^{[19-21]}$ ), o acoplamento eletrônico-vibracional torna-se importante e não é possível desacoplar o sistema de equações (1.6).

Geralmente, nos sistemas moleculares, os elementos $\hat{\Lambda}_{n n}(\mathbf{R})$ são muito menores que o potencial adiabático $\varepsilon_{n}(\mathbf{R})$. Portanto, obtém-se a aproximação de BornOppenheimer (ABO) da seguinte forma:

$$
\left[\hat{T}(\mathbf{R})+\varepsilon_{n}(\mathbf{R})\right] \chi_{m}(\mathbf{R})=E \chi_{n}(\mathbf{R})
$$

Nesta expressão, fica claro que a energia eletrônica $\varepsilon_{n}(\mathbf{R})$ será a energia potencial de interação no problema nuclear, ou seja, a superfície de energia potencial (SEP) que controla o movimento dos núcleos.

Assim, a aproximação de Born-Oppenheimer para sistemas moleculares, permite resolver o problema dos movimentos nucleares na molécula seguindo uma metodologia relativamente mais fácil. A completa separação entre o movimento dos núcleos e dos elétrons permite, em primeiro lugar, resolver o problema eletrônico em configurações com os núcleos fixos, obtendo-se os valores $\varepsilon_{n}(\mathbf{R})$ em função das coordenadas 
nucleares que definem a SEP do sistema. Uma vez resolvido o problema eletrônico, se resolveria o problema nuclear considerando $\varepsilon_{n}(\mathbf{R})$ como potencial de interação que controla o movimento dos núcleos.

\subsection{Cálculos ab initio}

Num sistema molecular formado por $n_{e}$ elétrons e $N$ núcleos, o hamiltoniano eletrônico numa configuração nuclear fixa pode ser escrito da seguinte forma:

$$
\hat{H}_{\mathrm{e}}=\sum_{i}^{n_{e}} \hat{h}(i)+V_{\mathrm{ee}}+V_{\mathrm{NN}}
$$

O segundo e terceiro termo dessa expressão são mostrados na equação (1.2), indicando respectivamente as interações elétron-elétron e núcleo-núcleo, enquanto o primeiro termo indica o hamiltoniano de um elétron (ou do caroço):

$$
\hat{h}(i)=-\frac{1}{2} \nabla_{i}^{2}+\sum_{k}^{N} \frac{Z_{k}}{R_{i k}}
$$

$R_{i k}=\left|\mathbf{r}_{i}-\mathbf{R}_{k}\right|$ e $r_{i j}$ têm o mesmo significado que na seção anterior. Portanto, a equação (1.3) fica da seguinte maneira:

$$
\left(\sum_{i}^{n} \hat{h}(i)+V_{\mathrm{ee}}+V_{\mathrm{NN}}\right) \Phi_{n}(\boldsymbol{r})=\varepsilon_{n} \Phi_{n}(\boldsymbol{r})
$$

as dependências de $\hat{h}(i), \varepsilon_{n}$ e $\Phi_{n}$ das coordenadas $\mathbf{R}$ foram omitidas por comodidade.

A resolução da equação (1.15) é normalmente chamada de cálculo ab initio. A sua resolução para sistemas moleculares necessita de métodos aproximados, alguns dos quais serão discutidos neste capítulo. 


\subsection{A aproximação de Hartree-Fock}

A resolução do problema eletrônico, em sistemas de muitos elétrons, pode ser abordada partindo da aproximação de partículas independentes. Assim, a função de onda é representada pelo produto de funções monoeletrônicas, chamadas de orbitais moleculares. Só que esse produto tem que cumprir o princípio de antissimetria da função de onda ${ }^{[22]}$, ou seja, a função de onda eletrônica deve ser antissimétrica ante a troca das coordenadas espaciais e de spin de dois elétrons quaisquer. Essa propriedade é satisfeita expressando a função de onda na forma do determinante de Slater:

$$
\Phi=\frac{1}{\sqrt{n !}}\left|\begin{array}{cccc}
\psi_{1}(1) & \psi_{1}(2) & \cdots & \psi_{1}(n) \\
\psi_{2}(1) & \psi_{2}(2) & \cdots & \psi_{2}(n) \\
\vdots & \vdots & \ddots & \vdots \\
\psi_{N}(1) & \psi_{N}(2) & \cdots & \psi_{N}(n)
\end{array}\right|
$$

onde as funções monoeletrônica $\psi_{i}$ representam os spin-orbitais moleculares, que são o produto da função espacial e da função de spin:

$$
\psi_{i}(j)=\phi_{i}\left(\mathbf{r}_{j}\right) \alpha\left(\omega_{j}\right) \quad \text { ou } \psi_{i}(j)=\phi_{i}\left(\mathbf{r}_{j}\right) \beta\left(\omega_{j}\right)
$$

As variáveis $\mathbf{r}_{j}$ e $\omega_{j}$ são as coordenadas espaciais e de spin do elétron $j$.

A aproximação de Hartree-Fock (HF) ${ }^{[23]}$ usa a função de onda (1.16) como função de tentativa no método variacional ${ }^{[22]}$ para descrever o estado fundamental de um sistema molecular qualquer. Dessa forma, os orbitais $\psi_{i}$ são otimizados para obter o melhor determinante de Slater que possa descrever esse estado eletrônico.

A função de onda, no cálculo variacional, é determinada encontrando o extremo do funcional energia:

$$
\varepsilon=\int \Phi^{*} \hat{H} \Phi \mathrm{d} \tau
$$

e o sucesso do método depende da função de tentativa escolhida. A função de onda (1.16) pode ser escrita da seguinte forma:

$$
\Phi=\hat{A}\left[\psi_{1}(1) \psi_{2}(2) \cdots \psi_{\mathrm{n}}(n)\right]
$$


onde o operador $\hat{A}$ é o operador de antissimetria seguinte:

$$
\hat{A}=\frac{1}{\sqrt{n}} \sum_{k=1}^{n !}(-1)^{k} \hat{P}
$$

sendo $\hat{P}$ o operador de permutações de duas partículas. O operador $\hat{A}$ satisfaz as seguintes relações:

$$
\hat{A}^{2}=\sqrt{n !} \hat{A}, \quad \hat{A}^{+}=\hat{A}, \quad \hat{A}\left(\frac{1}{r_{i j}}\right)=\left(\frac{1}{r_{i j}}\right) \hat{A}, \quad \hat{A} \hat{h}(i)=\hat{h}(i) \hat{A} .
$$

Usando a função de tentativa (1.19) e o hamiltoniano eletrônico (1.13) no cálculo variacional, a equação (1.18) fica da seguinte forma:

$$
\begin{aligned}
\varepsilon= & \underbrace{\frac{1}{\sqrt{n !}} \sum_{i} \int \hat{A}^{*}\left[\psi_{1}(1) \psi_{2}(2) \cdots \psi_{n}(n)\right]^{*} \hat{h}(i) \hat{A}\left[\psi_{1}(1) \psi_{2}(2) \cdots \psi_{n}(n)\right] \mathrm{d} \tau}_{I_{1}}+ \\
& +\underbrace{\frac{1}{2} \frac{1}{\sqrt{n !}} \sum_{i \neq j} \int \hat{A}^{*}\left[\psi_{1}(1) \psi_{2}(2) \cdots \psi_{n}(n)\right]^{*}\left(\frac{1}{r_{i j}}\right) \hat{A}\left[\psi_{1}(1) \psi_{2}(2) \cdots \psi_{n}(n)\right] \mathrm{d} \tau}_{I_{2}} .
\end{aligned}
$$

Agora, se os spin-orbitais moleculares $\psi_{k}$ não formassem um conjunto de funções ortonormais, eles poderiam ser transformados através de uma transformação unitária a um novo conjunto de spin-orbitais ortonormais $\left\{\psi_{k}^{\prime}\right\}$, onde as funções de onda antes e depois da transformação somente vão ser diferentes por um fator de fase ${ }^{[24]}$. Assim, sem perda de generalidade podemos considerar que os spin-orbitais $\psi_{k}$ formam um conjunto ortonormal. Portanto, aplicando as propriedades (1.21) e sabendo que $\int \psi_{i}(i) \psi_{j}(i) \mathrm{d}^{3} r_{i}=\delta_{i}^{j}, I_{1}$ e $I_{2}$ ficam:

$$
\begin{gathered}
I_{1}=\sum_{i=1}^{n_{e}} \int \psi_{i}(i) \hat{h}(i) \psi_{i}(i) \mathrm{d}^{3} \boldsymbol{r}_{i} \\
I_{2}=\frac{1}{2} \sum_{i, j}^{n_{e}}\left[\int \psi_{i}^{*}(i) \psi_{j}^{*}(j) \frac{1}{r_{i j}} \psi_{i}(i) \psi_{j}(j) \mathrm{d}^{3} \boldsymbol{r}_{i} \mathrm{~d}^{3} \boldsymbol{r}_{j}-\int \psi_{i}^{*}(i) \psi_{j}^{*}(j) \frac{1}{r_{i j}} \psi_{j}(i) \psi_{i}(j) \mathrm{d}^{3} \boldsymbol{r}_{i} \mathrm{~d}^{3} \boldsymbol{r}_{j}\right] .
\end{gathered}
$$


Escrevendo de forma mais simplificada, o funcional energia do sistema fica:

$$
\varepsilon=\sum_{i=1}^{n_{e}}\langle i|\hat{h}| i\rangle+\frac{1}{2} \sum_{i, j}^{n_{e}}\langle i j \| i j\rangle
$$

onde $|i\rangle$ indica o spin-orbital $\psi_{i}(i)$ e o segundo termo engloba as integrais de dois elétrons

$$
\langle i j \| i j\rangle=\langle i j \mid i j\rangle-\langle i j \mid j i\rangle
$$

com

$$
\langle i j \mid k l\rangle=\int \psi_{i}^{*}(1) \psi_{j}^{*}(2) \frac{1}{r_{12}} \psi_{k}(1) \psi_{l}(2) \mathrm{d} \mathbf{r}_{1} \mathrm{~d} \mathbf{r}_{2} .
$$

O primeiro termo da direita na equação (1.24) é a repulsão coulombiana entre dois elétrons nos orbitais $i$ e $j$. O segundo termo é a interação de troca entre os elétrons nesses orbitais. Essa é uma interação que não tem análogo clássico e se origina da indistinguibilidade das partículas, que impõe a antissimetria da função de onda.

O passo seguinte é encontrar os spin-orbitais moleculares $\psi_{i}$ que minimizam o funcional $\varepsilon$ mantendo a ortogonalidade entre eles. Para isso são usados os multiplicadores de Lagrange, onde agora o funcional a minimizar é o seguinte:

$$
\zeta=\sum_{i=1}^{n_{e}}\langle i|\hat{h}| i\rangle+\frac{1}{2} \sum_{i, j}^{n_{e}}\langle i j \| i j\rangle+\sum_{i, j}^{n_{e}} \lambda_{i j}\left(\langle i \mid j\rangle-\delta_{i j}\right)
$$

sendo $\lambda_{i j}$ são os multiplicadores de Lagrange. Minimizando este funcional, (ver detalhes na Ref. ${ }^{[24]}$ ) se obtém:

$$
\hat{f}(1) \psi_{i}(1)=\frac{1}{2} \sum_{j=i}^{n_{e}} \lambda_{i j} \psi_{j}(1)
$$

onde $f$ é o operador de Fock:

$$
\hat{f}(1)=\hat{h}(1)+\sum_{i=1}^{n_{e}}\left[\hat{\mathrm{J}}_{i}(1)-\hat{\mathrm{K}}_{i}(1)\right]
$$

com $\hat{\mathbf{J}}_{i}$ e $\hat{\mathrm{K}}_{i}$ definidos da seguinte forma

$$
\hat{\mathbf{J}}_{j}(1) \psi_{i}(1)=\left[\int \psi_{j}^{*}(2) \frac{1}{r_{12}} \psi_{j}(2) \mathrm{d} \mathbf{r}_{2}\right] \psi_{i}(1)
$$




$$
\hat{\mathrm{K}}_{j}(1) \psi_{i}(1)=\left[\int \psi_{j}^{*}(2) \frac{1}{r_{12}} \psi_{i}(2) \mathrm{d} \mathbf{r}_{2}\right] \psi_{j}(1) .
$$

Diagonalizando a matriz hermitiana $\lambda$ da equação (1.27) através de uma transformação unitária, obtém-se um novo conjunto de spin-orbitais que diferem dos anteriores somente por um fator de fase ${ }^{[24]}$. Dessa forma, obtém-se a equação de Hartree-Fock para a determinação dos spin-orbitais $\psi_{i}$, da seguinte forma:

$$
\hat{f}(1) \psi_{i}(1)=\varepsilon_{i} \psi_{i}(1)
$$

Esse novo conjunto $\psi_{i}$ são os autovetores do operador de Fock, e também são os orbitais que minimizam o funcional energia do sistema (1.23). Os autovalores $\varepsilon_{i}$ são interpretados como sendo as energias associadas aos orbitais moleculares, pois o operador $\hat{f}$ representa todas as interações que sente cada elétron.

O operador $\hat{\mathbf{J}}_{j}$, ou operador de Coulomb, representa o potencial médio gerado por um elétron no orbital $j$ e o operador de troca $\hat{\mathrm{K}}_{j}$ faz a troca entre os elétrons do orbital $j$ e do orbital em que o operador atua. Somando sobre todos os orbitais ocupados, temos que cada elétron sentirá um campo médio gerado pelos outros elétrons. Se deve notar que o campo médio depende dos spin-orbitais ocupados, portanto, a equação de HartreeFock tem que ser resolvida iterativamente. No começo um conjunto de orbitais iniciais é usado para construir o campo inicial que possibilita a obtenção de um novo conjunto de spin-orbitais e um novo valor do funcional energia do sistema. Esse processo é repetido iterativamente até a convergência da energia do sistema, quando é atingido um campo autoconsistente.

A aproximação de Hartree-Fock, sendo uma aproximação de campo médio, não considera a energia de correlação eletrônica. Portanto, melhoras na energia do sistema têm que ser obtidas com métodos além de Hartree-Fock.

\subsection{Cálculos além de Hartree-Fock}

Os resultados dos cálculos HF têm indicado em muitos casos bons resultados em vários tipos de problemas, como otimizações de geometrias de equilíbrio de moléculas. Além 
disso, é sabido que a energia de HF representa a maior parte da energia total do sistema, usualmente entre o 95 e $99 \%{ }^{[18]}$. Porém, na grande maioria dos problemas da físicoquímica a energia de correlação eletrônica é extremadamente importante, pois é da ordem das magnitudes de interesse, sendo necessário obter energias do sistema além da energia de HF. A energia de correlação é definida através da diferença entre a energia exata do sistema e a energia de HF:

$$
E_{\text {corr }}=E_{\text {exata }}-E_{\mathrm{HF}} \text {. }
$$

A seguir serão discutidos alguns dos métodos usados para obter parte da energia de correlação dos sistemas moleculares.

\subsubsection{Métodos de interação de configurações e campo autoconsistente multiconfiguracional}

Uma forma sistemática de incluir energia de correlação é expandir a função de onda eletrônica em mais de um determinante de Slater

$$
\Phi=\sum_{k} c_{k} D_{k}
$$

neste caso, $D_{0}$ seria o determinante de Slater da função de onda para o estado de referência, composto pelos orbitais moleculares mais baixos, enquanto $D_{k}(K>0)$ são determinantes de Slater com um ou mais elétrons em orbitais virtuais. Desta forma, a função de onda (1.33), tem contribuição de varias configurações possíveis do sistema quântico. Essa metodologia é chamada de interação de configurações ${ }^{[24,25]}$. Os orbitais moleculares, ocupados e virtuais, usados nestes cálculos, geralmente são os orbitais moleculares obtidos de cálculos HF (orbitais SCF) e os coeficientes $c_{k}$ do cálculo de interação de configurações são otimizados no cálculo variacional. Os cálculos de interação de configurações são classificados pelo número de excitações eletrônicas feitas para orbitais virtuais. Se somente um elétron é excitado de cada vez para formar um novo determinante de Slater, é chamado de interação de configurações de excitações simples (CIS) ${ }^{[26]}$. Os cálculos CIS podem dar energias aproximadas dos estados eletrônico excitados, mas não modificam a energia do estado fundamental, pois, 
segundo o teorema de Brillouin ${ }^{[24]}$ os determinantes formados por excitações simples não interagem com o estado de referência $D_{0}$. Excitações simples e duplas (CISD) ${ }^{[26]}$ sim permitem calcular parte da energia de correlação eletrônica, mas, ao truncar as excitações só a simples e duplas o método vira não consistente com o tamanho do sistema ${ }^{[24]}$. Uma variante de considerar as excitações duplas é usando elas de forma perturbativa no espaço das excitações simples. Este método é conhecido como CIS(D) [27], é consistente com o tamanho do sistema molecular e é muito usado no estudo de estados excitados como uma correção aos cálculos CIS. Este método, CIS(D), é amplamente usado nos trabalhos apresentados nesta tese nos cálculos de energias de transição. Excitações triplas (CISDT) ${ }^{[26]}$ ou quádruplas (CISDTQ) ${ }^{[26]}$ são usadas somente quando for desejado resultados muito precisos da energia do sistema. Cálculos de interação de configurações incluindo todas as excitações possíveis são chamados de full-configuration interaction (FCI). Cálculos FCI usando uma base infinita dariam resultados exatos do sistema quântico estudado, mas cálculos desse tipo, mesmo para base finita, são raramente feitos pelo grande esforço computacional.

A convergência dos métodos interação de configurações é relativamente lenta, portanto, têm que ser incluídos muitos orbitais SCF para obter bons resultados. Outra forma de obter bons resultados nos cálculos de interação de configurações é usando o método de campo autoconsistente multiconfiguracional (MCSCF) ${ }^{[24,25]}$. Nesses cálculos a função de onda é representada da mesma forma que no cálculo de interação de configurações (equação (1.33)), mas no cálculo variacional são otimizados tanto os coeficientes $c_{k}$, da interação de configurações, quanto os orbitais SCF. Os orbitais SCF são modificados no cálculo variacional mudando os coeficientes que definem o orbital molecular em função das funções-base. Desta forma, pode-se obter melhores resultados incluindo relativamente poucos orbitais SCF. Nos cálculos MCSFC, os orbitais moleculares são divididos em três grupos, inativos, ativos e virtuais ${ }^{[28]}$. Os orbitais inativos são mantidos duplamente ocupados e os virtuais são orbitais desocupados. Nos orbitais ativos são distribuídos os elétrons ativos para gerar as diferentes configurações. Se os elétrons ativos são distribuídos de todas as formas possíveis nos orbitais ativos, o cálculo é conhecido como CASSCF (Complete Active Space Self Consistent Field). Se o número de excitações dentro do espaço ativo é restringido, então é um cálculo RASSCF (Restricted Active Space Self Consistent Field). Comumente os orbitais ativos são os orbitais moleculares de valência que derivam dos orbitais atômicos dos átomos que formam a molécula. O método CASSCF é amplamente usado nos trabalhos 
apresentados nesta tese no estudo de propriedades e otimização de geometrias de estados excitados.

\subsubsection{Métodos perturbativos (Rayleigh-Schrödinger)}

Os métodos perturbativos, em geral, permitem descrever um sistema quântico determinado a partir de outros sistemas quânticos menos complexos. Nesta seção será discutida a teoria de perturbação de Rayleigh-Schrödinger ${ }^{[22]}$, que é amplamente usada na físico-química computacional. Posteriormente faremos uma pequena discussão de como ela é aplicada nos métodos perturbativos mais difundidos, a teoria de perturbação de Møller Plesset (MP) ${ }^{[29]}$ e o método perturbativos de segunda ordem de espaço ativo completo (CASPT2) ${ }^{[26,30]}$.

O problema de autovalores a ser resolvido é o seguinte:

$$
\hat{H} \psi_{n}=E_{n} \psi_{n},
$$

mas, na teoria de Rayleigh-Schrödinger o hamiltoniano é dividido em duas partes, o hamiltoniano não perturbado mais a perturbação,

$$
\hat{H}=\hat{H}_{0}+\hat{H}^{\prime}
$$

sendo $H_{0}$ o hamiltoniano não perturbado com autovalores e autofunções conhecidos:

$$
\hat{H}_{0} \psi_{n}^{(0)}=E_{0}^{(0)} \psi_{n}^{(0)}
$$

Supondo que a perturbação se aplica gradualmente desde o sistema não perturbado até o sistema perturbado, o hamiltoniano poderia ser representado da seguinte forma:

$$
\hat{H}=\hat{H}_{0}+\lambda \hat{H}^{\prime}
$$

onde $\lambda$ vai desde zero até um. Assim, tanto as autofunções quanto os autovalores de $\hat{H}$ vão depender do parâmetro $\lambda$. Ou seja, $\psi_{n}=\psi_{n}(\lambda, q)$ e $E_{n}=E_{n}(\lambda)$, sendo $q$ as coordenadas do sistema. Fazendo uma expansão em series de Taylor dos autovalores e autofunções, fica: 


$$
\begin{gathered}
\psi_{n}=\psi_{n}^{(0)}+\lambda \psi_{n}^{(1)}+\lambda^{2} \psi_{n}^{(2)}+\cdots+\lambda^{k} \psi_{n}^{k}+\cdots \\
E_{n}=E_{n}^{(0)}+\lambda E_{n}^{(1)}+\lambda^{2} E_{n}^{(2)}+\cdots+\lambda^{k} E_{n}^{k}+\cdots
\end{gathered}
$$

onde $\psi_{n}^{(k)}$ e $E_{n}^{(k)}$ são as correções de ordem $k$ da função de onda e da energia respectivamente.

Substituindo (1.38) e (1.39) em (1.34), fazendo uma diagonalização intermediaria [24] entre as autofunções $\psi_{n}^{(0)}$ e $\psi_{n}^{(k)}$, e igualando os termos da mesma potencia em $\lambda$, vamos ter:

$$
\begin{gathered}
\hat{H}_{0} \psi_{n}^{(0)}=E_{n}^{(0)} \psi_{n}^{(0)} \\
\hat{H}_{0} \psi_{n}^{(1)}+\hat{H}^{\prime} \psi_{n}^{(0)}=E_{n}^{(0)} \psi_{n}^{(1)}+E_{n}^{(1)} \psi_{n}^{(0)} \\
\hat{H}_{0} \psi_{n}^{(2)}+\hat{H}^{\prime} \psi_{n}^{(1)}=E_{n}^{(0)} \psi_{n}^{(2)}+E_{n}^{(1)} \psi_{n}^{(1)}+E_{n}^{(2)} \psi_{n}^{(0)} \\
\hat{H}_{0} \psi_{n}^{(3)}+\hat{H}^{\prime} \psi_{n}^{(2)}=E_{n}^{(0)} \psi_{n}^{(3)}+E_{n}^{(1)} \psi_{n}^{(2)}+E_{n}^{(2)} \psi_{n}^{(1)}+E_{n}^{(3)} \psi_{n}^{(0)}
\end{gathered}
$$

Multiplicando cada uma das equações anteriores por $\psi_{n}^{*(0)}$, integrando por todo o espaço e usando a condição de ortogonalidade entre as autofunções $\psi_{n}^{(0)}$ e $\psi_{n}^{(k)}$, fica:

$$
\begin{aligned}
& E_{n}^{(0)}=\left\langle\psi_{n}^{(0)}\left|\hat{H}_{0}\right| \psi_{n}^{(0)}\right\rangle \\
& E_{n}^{(1)}=\left\langle\psi_{n}^{(0)}\left|\hat{H}^{\prime}\right| \psi_{n}^{(0)}\right\rangle \\
& E_{n}^{(2)}=\left\langle\psi_{n}^{(0)}\left|\hat{H}^{\prime}\right| \psi_{n}^{(1)}\right\rangle
\end{aligned}
$$

Dos resultados anteriores pode-se ver que as correções de ordem $k$ da energia vão depender somente das funções de onda de ordem inferior. Como indica a equação (1.45), a correção de primeira ordem da energia é o valor médio da perturbação. Para determinar a correção de primeira ordem da função de onda, expandimos a função de onda no conjunto completo de autofunções não perturbadas:

$$
\psi_{n}^{(1)}=\sum_{m} a_{m} \psi_{m}^{(0)} \quad \text { onde } \quad a_{m}=\left\langle\psi_{m}^{(0)} \mid \psi_{n}^{(1)}\right\rangle .
$$


Agora, multiplicando (1.41) por $\psi_{m}^{*(0)}$, integrando e usando a expansão (1.47) obtém-se a correção de primeira ordem da função de onda:

$$
\psi_{n}^{(1)}=\sum_{m \neq n} \frac{\left\langle\psi_{m}^{(0)}\left|\hat{H}^{\prime}\right| \psi_{n}^{(0)}\right\rangle}{E_{n}^{(0)}-E_{m}^{(0)}} \psi_{m}^{(0)}
$$

Usando esta correção de primeira ordem da função de onda na equação (1.46), pode-se determinar a correção de segunda ordem da energia, ficando:

$$
E_{n}^{(2)}=\sum_{m \neq n} \frac{\left|\left\langle\psi_{m}^{(0)}\left|\hat{H}^{\prime}\right| \psi_{n}^{(0)}\right\rangle\right|^{2}}{E_{n}^{(0)}-E_{m}^{(0)}}
$$

As correções de ordens superiores podem ser obtidas seguindo a metodologia descrita, detalhes podem ser encontradas em textos mais avançados como o Szabo e Ostlund ${ }^{[24]}$.

Como foi comentado anteriormente, a teoria de perturbação de RayleihgSchrödinger ${ }^{[22]}$ é aplicada nos métodos perturbativos de Møller Plesset ${ }^{[29]}$ e CASPT2 [26, 30]. No método perturbativo de Møller Plesset o hamiltoniano não perturbado é a soma por todos os elétrons do operador de Fock (equação 1.28), obtendo-se:

$$
\hat{H}_{0}=\sum_{i=1}^{n} \hat{f}_{i}=\sum_{i=1}^{n}\left(\hat{h}_{i}+\sum_{j=1}^{n}\left(\hat{J}_{i j}-\hat{K}_{i j}\right)\right) \text {. }
$$

A perturbação é determinada subtraindo o hamiltoniano não perturbado do hamiltoniano total:

$$
\hat{H}^{\prime}=\hat{H}-\hat{H}_{0}=\sum_{i=1}^{n} \hat{h}_{i}+V_{e e}-\sum_{i=1}^{n}\left(\hat{h}_{i}+\sum_{j=1}^{n}\left(\hat{J}_{i j}-\hat{K}_{i j}\right)\right)=V_{e e}-2\left\langle V_{e e}\right\rangle .
$$

Dessa expressão pode-se ver que a correção de primeira ordem da energia, que seria o valor médio da perturbação é a energia de Hartree-Fock. Portanto, a correção para a energia de correlação começa com a correção de segunda ordem, MP2 (MP, de Møller Plesset). O método MP2 é muito usado nesta tese na otimização de geometrias correspondentes ao estado fundamental. As correções de ordem superior também podem ser obtidas, como MP3, MP4 e assim por diante. Infelizmente, as perturbações de ordem superior, geralmente não seguem um comportamento monotônico convergente a um 
valor limite, onde cada correção deveria manter o mesmo sinal e diminuir em magnitude.

Outra forma de usar o método perturbativo é usando a função de onda obtida de cálculos CASSCF como a função de onda de ordem zero. Este é o caso do método CASPT2 (ou CASPTn) ${ }^{[26,30]}$. Uma dificuldade deste método é o fato de a função de onda CASSCF não ser autofunção do hamiltoniano não perturbado (1.50), portanto, $\hat{H}_{0}$ tem que ser redefinido. A solução proposta para resolver esse problema ${ }^{[30]}$ foi projetar o hamiltoniano $\hat{H}_{0}$ no espaço das funções de interação de configurações. Esse procedimento é feito com a ajuda dos seguintes operadores de projeção. $\hat{P}_{0}$ projeta no espaço da função de onda de referência inicial $\left(V_{0}\right)$, enquanto $\hat{P}_{K}$ projeta no espaço definido pelo complemento ortogonal do espaço $V_{0}$, pertencentes ao espaço ativo usado para gerar a função de onda CAS. $\hat{P}_{S D}$ projeta no espaço $V_{S D}$, formado por todos os estados obtidos das substituições simples e duplas partindo de $V_{0}$. E $\hat{P}_{T Q}$ vai projetar no espaço $V_{T Q}$ que contem todas as excitações de maior ordem não incluídas nos espaços anteriores. Usando esses operadores de projeção sobre o operador de Fock CASSCF, $\hat{F}$ [30], obtém-se o hamiltoniano não perturbado do método CASPTn:

$$
\hat{H}_{0}^{\mathrm{CASPT}}=\hat{P}_{0} \hat{F} \hat{P}_{0}+\hat{P}_{K} \hat{F} \hat{P}_{K}+\hat{P}_{S D} \hat{F} \hat{P}_{S D}+\hat{P}_{T Q} \hat{F} \hat{P}_{T Q}
$$

De forma geral, essa é a metodologia usada na obtenção do hamiltoniano não perturbado nos cálculos CASPTn. Aplicando a teoria de perturbação antes descrita até segunda ordem nesse hamiltoniano e usando a função de onda CASSCF como função de ordem zero, obtêm-se energias CASPT2 que contém uma parte significativa da energia de correlação eletrônica. Este método é usado em vários dos trabalhos apresentados para calcular as energias de estados eletrônicos e energias de transição.

\subsection{Decaimento não radiativo}

Nesta seção serão discutidos alguns elementos do decaimento não radiativo em sistemas moleculares, dada a importância que têm em dois dos trabalhos apresentados nesta tese. 
Após a transição de absorção para os estados eletrônicos excitados, geralmente o sistema fica excitado vibracionalmente. Antes que o sistema possa se mover pela superfície de energia potencial do estado excitado, a energia vibracional é distribuída nos modos normais de vibração. Logo depois, podem ocorrer vários processos, começando pelo esfriamento vibracional e relaxação da geometria para a configuração de equilíbrio. Neste processo, desconsiderando fotoprodutos de reação, o sistema pode decair por fluorescência para o estado fundamental ou podem acontecer processos de decaimento não radiativo, como conversão interna (CI), intersystem crossing (ISC) ou cruzamentos entre superfícies de energia potencial (interseções cônicas).

\subsubsection{Conversão interna}

A conversão interna é uma transição não radiativa que pode acontecer após a absorção eletrônica para estados excitados. Essa transição não radiativa está associada a transições entre estados vibracionais isoenergéticos ( $v$ e $v^{\prime}$ ), correspondentes a estados eletrônicos diferentes $(n$ e $m$ ) de igual multiplicidade de spin, podendo-se representar da seguinte forma: $\mathrm{S}_{n}^{v} \rightarrow \mathrm{S}_{m}^{v^{\prime}}$ ou $\mathrm{T}_{n}^{v} \rightarrow \mathrm{T}_{m}^{v^{\prime}}$. Ou seja, o sistema muda entre estados eletrônicos de igual multiplicidade através de estados vibracionais ressonantes ${ }^{[19]}$. A energia de excitação nestes casos é transformada em calor.

Geralmente, segundo a regra de Kasha ${ }^{[31]}$, a CI entre estados eletrônicos excitados, como $\mathrm{S}_{n}^{v} \rightarrow \mathrm{S}_{1}^{v^{\prime}}$ ou $\mathrm{T}_{n}^{v} \rightarrow \mathrm{T}_{1}^{v^{\prime}}$, é muito mais eficientes do que a CI entre o primeiro estado excitado e o estado fundamental. Por causa disso na maioria dos sistemas moleculares a luminescência é observada somente de transições desde o estado eletrônico excitado mais baixo.

De forma geral, nos sistemas moleculares se observa que a eficiência da CI entre os estados $\mathrm{S}_{1}^{\nu} \rightarrow \mathrm{S}_{0}^{v^{\prime}}$ depende da diferença energética entre os estados $\mathrm{S}_{1}$ e $\mathrm{S}_{0}(\Delta E)$, obtendo-se a seguinte lei empírica (energy-gap law) ${ }^{[32]}$ para a constante de velocidade da CI:

$$
k_{\mathrm{CI}}=10^{13} \mathrm{e}^{-\alpha \Delta E}
$$

Sendo $\alpha$ uma constante de proporcionalidade aproximadamente igual a $4.85 \mathrm{eV}^{-1}$. 
Outra forma de conversão interna muito eficiente são as intersecções cônicas, que são regiões de degenerescência entre estados eletrônicos que possuem igual multiplicidade eletrônica. As intersecções cônicas não serão discutidas, pois, não têm relevância nos trabalhos apresentados.

\subsubsection{Intersystem crossing}

A transição não radiativa entre estados eletrônicos de diferente multiplicidade é possível através da inversão do spin, dada pela interação spin-órbita. Transições deste tipo, $\mathrm{S}_{1}^{v} \rightarrow \mathrm{T}_{n}^{v^{\prime}}$, podem ocorrer entre estados vibracionais ressonantes correspondentes a estados eletrônicos diferentes e são favorecidas segundo a magnitude do acoplamento spin-órbita. Após a transição para o estado eletrônico $T_{n}$ pode ocorrer conversão interna entre os estados tripletos até o estado eletrônico mais baixo $T_{1}$.

Segundo a regra de ouro de Fermi, a probabilidade de transição entre um estado eletrônico inicial $I$ e um estado final $F$ cumpre a seguinte proporcionalidade ${ }^{[19]}$ :

$$
T_{I \rightarrow F} \sim\left\langle{ }^{3} \Psi_{F}\left|\hat{\boldsymbol{H}}_{\mathrm{SO}}\right|{ }^{1} \Psi_{I}\right\rangle^{2}
$$

onde $\Psi_{I}$ e $\Psi_{F}$ são as funções de onda de cada um dos estados eletrônicos e $\hat{\boldsymbol{H}}_{\mathrm{SO}}$ representa o hamiltoniano da interação spin-órbita. Na aproximação adiabática, já comentada na seção (1.1), o movimento dos núcleos pode ser separado do movimento dos elétrons, ficando

$$
\Psi=\psi(\mathbf{R} ; \mathbf{r}) \chi(\mathbf{R})
$$

onde, novamente, $\psi(\mathbf{R} ; \mathbf{r})$ e $\chi(\mathbf{R})$ são as funções de onda eletrônica e nuclear respectivamente. As coordenadas $\mathbf{R}$ representam as coordenadas dos núcleos enquanto $\mathbf{r}$ representa as coordenadas eletrônicas. Substituindo esta expressão em (1.54), se obtém:

$$
T_{I \rightarrow F} \sim\left\langle{ }^{3} \Psi_{F}\left|\hat{\boldsymbol{H}}_{\mathrm{SO}}\right|{ }^{1} \Psi_{I}\right\rangle^{2} \approx\left\langle{ }^{3} \psi_{F}\left|\hat{\boldsymbol{H}}_{\mathrm{SO}}\right|{ }^{1} \psi_{I}\right\rangle^{2}\left\langle\chi_{F} \mid \chi_{I}\right\rangle^{2} .
$$


Aqui foram omitidas as dependências com $\mathbf{R}$ e $\mathbf{r}$ das funções de onda. A expressão $\left\langle\chi_{F} \mid \chi_{I}\right\rangle$ é a integral de overlap do movimento dos núcleos em cada um dos estados eletrônicos.

O hamiltoniano da interação spin-órbita pode ser representado para átomos leves através do hamiltoniano de Breit-Pauli ${ }^{[33]}$ :

$$
\hat{\boldsymbol{H}}_{\mathrm{SO}}^{\mathrm{BP}}=\frac{1}{2 c^{2}}\left[\sum_{i} \sum_{\alpha} Z_{\alpha} \hat{\boldsymbol{s}}_{\boldsymbol{i}} \cdot\left(\frac{\boldsymbol{r}_{i \alpha}}{\boldsymbol{r}_{i \alpha}^{3}} \times \hat{\boldsymbol{p}}_{i}\right)-\sum_{i \neq j}\left(\frac{\boldsymbol{r}_{i j}}{\boldsymbol{r}_{i j}^{3}} \times \hat{\boldsymbol{p}}_{i}\right) \cdot\left(\hat{\boldsymbol{s}}_{i}+2 \hat{\boldsymbol{s}}_{j}\right)\right] .
$$

Nessa expressão, $Z_{\alpha}$ indica o número atômico do núcleo $\alpha, \hat{\boldsymbol{p}}_{i}$ e $\hat{\boldsymbol{s}}_{\boldsymbol{i}}$ são os operadores do momentum e momentum angular de spin do elétron $i$ e $\boldsymbol{r}$ representa o vetor distância entre pares de elétrons $\left(\boldsymbol{r}_{i j}\right)$ ou elétron-núcleo $\left(\boldsymbol{r}_{i \alpha}\right)$. O primeiro termo deste hamiltoniano representa a interação entre os momentos magnéticos associados ao spin de cada elétron e ao movimento orbital dele em relação aos núcleos atômicos. $\mathrm{O}$ segundo termo considera a interação entre pares de elétron de um mesmo orbital e de orbitais diferentes.

Para fazer viável o cálculo da interação spin-órbita em sistemas moleculares, dada pela integral $\left\langle{ }^{3} \psi_{F}\left|\hat{\boldsymbol{H}}_{\mathrm{SO}}\right|{ }^{1} \psi_{I}\right\rangle$, é muito usada a aproximação de campo médio proposta por Hess et al. ${ }^{[34]}$. Nessa aproximação o hamiltoniano (1.57) é aproximado por um hamiltoniano efetivo de um elétron, onde as contribuições dos termos de dois elétrons são aproximadamente calculadas sobre os elétrons de valência no campo gerado pelos outros elétrons. Alem disso, somente são consideradas as integrais de um elétron com dois centros e dois elétrons com um centro. Mais detalhes podem ser encontrados na Ref. ${ }^{[34]}$.

Existem umas regras de seleção para as transições de intersystem crossing, conhecidas como regras de El-Sayed ${ }^{[35]}$. Segundo estas regras de seleção, são permitidas transições entre estados eletrônicos de diferente simetria espacial. Por exemplo, são permitidas transições do tipo:

$$
{ }^{1}\left(\mathrm{n}, \pi^{*}\right) \rightarrow^{3}\left(\pi, \pi^{*}\right) \quad \text { e } \quad{ }^{3}\left(\mathrm{n}, \pi^{*}\right) \rightarrow^{1}\left(\pi, \pi^{*}\right)
$$

e proibidas transições de tipo:

$$
{ }^{1}\left(\mathrm{n}, \pi^{*}\right) \rightarrow^{3}\left(\mathrm{n}, \pi^{*}\right), \quad \text { e } \quad{ }^{1}\left(\pi, \pi^{*}\right) \rightarrow^{3}\left(\pi, \pi^{*}\right)
$$


O valor da constante de velocidade do ISC, $k_{\mathrm{ISC}}$, varia entre $10^{7}$ e $10^{11} \mathrm{~s}^{-1}$, dependendo da magnitude do acoplamento spin-órbita, e do overlap do movimento vibracional dos núcleos em cada um dos estado eletrônicos. 


\section{Capítulo 2}

\section{Superfícies de energia potencial e método de trajetórias quase-clássicas}

Neste capítulo descreveremos a metodologia usada no estudo de reações usando o método de trajetórias quase-clássicas. Será discutida brevemente a relevância da superfície de energia potencial nestes casos, assim como a metodologia DMBE, amplamente usada na construção da SEP. O modelo utilizado para descrever colisões bi-moleculares, assim como a função de excitação para reações de tipo captura serão também apresentados. Finalmente, será discutida a forma de gerar as condições iniciais da colisão no método de trajetórias quase-clássicas.

\subsection{Superfície de energia potencial DMBE}

A aproximação de Born-Oppenheimer (seção 1.1), em princípio, oferece uma metodologia para resolver o problema do movimento dos núcleos atômicos em sistemas moleculares. Segundo a equação (1.12), $\varepsilon_{n}(\mathbf{R})$ é a energia potencial de interação no problema nuclear, que normalmente é calculada por métodos ab initio para configurações fixas dos núcleos atômicos. Mesmo quando esses cálculos possam oferecer informação sobre a primeira e segunda derivada de $\varepsilon_{n}(\mathbf{R})$, uma representação 
analítica ou numérica da SEP global pode ser bastante conveniente em muitos casos ${ }^{[36,}$ 37].

No estudo de propriedades espectroscópicas é suficiente estudar algumas regiões próximas a certas configurações de interesse. Porém, em estudos de dinâmica, é preciso explorar grandes áreas da SEP ${ }^{[38]}$. Funções como a função de Morse e a expansão de muitos corpos ${ }^{[39]}$ são exemplos de funções que simulam a topologia da SEP e que são usadas em interpolações locais e globais ${ }^{[37,40]}$.

Um método muito usado para representar a SEP global de sistemas moleculares é a expansão de muitos corpos ${ }^{[39]}$ (MBE). A idéia principal deste método é descrever a energia de interação de um sistema molecular somando as contribuições de cada fragmento poliatômico que compõe o sistema molecular. A SEP de um sistema molecular de $n$ átomos se representaria neste esquema da seguinte forma:

$$
V_{\mathrm{ABC} . . \mathrm{N}}(\mathbf{R})=\sum V_{\mathrm{AB}}^{(2)}\left(R_{\mathrm{AB}}\right)+\sum V_{\mathrm{ABC}}^{(3)}\left(R_{\mathrm{AB}}, R_{\mathrm{AC}}, R_{\mathrm{BC}}\right)+\cdots+V_{\mathrm{ABC} . . \mathrm{N}}^{(n)}(\mathbf{R})
$$

Cada somatória indica a soma de todos os fragmentos de $n$-corpos correspondentes a cada termo dessa expressão. $\mathrm{O}$ termo $V_{\mathrm{AB}}^{(2)}\left(R_{\mathrm{AB}}\right)$ representa a energia de cada fragmento diatômico, que depende da distância entre os dois átomos e tende a zero quando a distância $R_{\mathrm{AB}}$ vai para o infinito. $V_{\mathrm{ABC}}^{(3)}\left(R_{\mathrm{AB}}, R_{\mathrm{AC}}, R_{\mathrm{BC}}\right)$ é o termo energético de cada fragmento triatômico que vai depender das distâncias interatômicas $R_{\mathrm{AB}}, R_{\mathrm{AC}}$ e $R_{\mathrm{BC}}$, cada um destes termos tende a zero quando um dos átomos é afastado infinitamente dos outros dois. O último termo representa a energia de $n$-corpos, esse termo é zero se um dos átomos é afastado para o infinito e depende das 3N-6 coordenadas internas do sistema molecular.

O método MBE oferece uma estratégia para representar a SEP global de sistemas moleculares poliatômicos relativamente grandes. Nesta metodologia todos os limites assintóticos são satisfeitos por definição e normalmente são reusadas funções MBE já construídas na construção da SEP de um sistema maior. Desta forma, se obtém uma função analítica que representa a SEP em todas as configurações possíveis do sistema molecular, tanto nas regiões onde se manifestam as interações de curto alcance como as de longo alcance. Um passo adiante em relação ao método MBE foi proposto por Varandas ${ }^{[40-43]}$, onde cada um dos termos da expansão (2.1) é dividido em duas partes, uma que descreve as interações de curto alcance, chamadas de extended Hartree-Fock e 
outra que descreve as de longo alcance, ou correlações dinâmicas. Este método, conhecido como expansão dupla de muitos corpos (DMBE), permite descrever as regiões de curto alcance através de funções polinômicas muito precisas, enquanto que as interações de longo alcance são descrita fenomenologicamente através das expansões multipolares ${ }^{[44,45]}$.

\subsection{Trajetórias clássicas}

Uma vez que o problema eletrônico é resolvido e é corretamente representado através de uma SEP, as reações químicas podem ser entendidas como o movimento dos núcleos sob a ação dessa energia potencial. Assim, podem ser usados métodos clássicos ou quânticos no estudo da dinâmica de reações químicas. Uma alternativa no estudo da dinâmica de sistemas moleculares é calcular a energia potencial, gradientes e eventualmente constantes de força on the fly. Desta forma, poucas vezes podem ser usados novamente os cálculos de mecânica quântica já feitos num outro estudo de dinâmica de reação. Além disso, geralmente o custo computacional é consideravelmente maior nos estudos de dinâmica on the fly, pois na construção de uma SEP grandes regiões são descritas fenomenologicamente através das expansões multipolares.

As trajetórias clássicas representam o limite de processos de dispersão de altas energias e partículas pesadas ${ }^{[37,46]}$. Nestas condições, as trajetórias clássicas são uma boa aproximação das reações químicas, onde algumas correção quânticas ${ }^{[47] \text { são }}$ requeridas em certas condições. Um conjunto de trabalhos realizados por Karplus et al. [48-50] têm indicado que o tratamento quântico e clássico de um mesmo sistema molecular não mostra diferenças significativas sempre que as energias translacionais não sejam muito baixas. Quando estas energias são muito baixas, os efeitos quânticos são mais significativos e têm sido obtidas algumas discrepâncias entre o tratamento clássico e o quântico ${ }^{[37]}$.

No estudo de trajetórias clássicas o movimento dos núcleos atômicos é simulado resolvendo numericamente as equações clássicas de Hamilton ${ }^{[51]}$ :

$$
\frac{\partial H}{\partial q_{i}}=-\frac{d p_{i}}{d t}, \quad \frac{\partial H}{\partial p_{i}}=\frac{d q_{i}}{d t}
$$


A função hamiltoniana $H$ é a soma da energia cinética $T(p, q)$ e a energia potencial $V(q)$, ou seja

$$
H=T(p, q)+V(q)
$$

onde a função $V(q)$ é a superfície de energia potencial do sistema estudado. As trajetórias calculadas contêm informação detalhada da reação estudada e calculando um conjunto delas pode-se tirar informações estatísticas que permitem determinar magnitudes como as constantes de velocidade de reação.

\subsection{Modelo quase-clássico para reações bi-}

\section{moleculares}

O método de trajetórias quase-clássicas ${ }^{[49]}$ é baseado na resolução clássica das equações de movimento, mas ao mesmo tempo, as condições iniciais das colisões levam em consideração os estados quânticos ro-vibracionais dos reagentes. Igualmente, os estados quânticos ro-vibracionais dos produtos podem ser determinados identificando os números quânticos que melhor descrevem os movimentos ro-vibracionais.

Consideraremos, a seguir, alguns conceitos básicos das colisões bi-moleculares. Sejam A e B dois reagentes que se aproximam com uma velocidade relativa inicial $v_{r e l} \mathrm{e}$ um parâmetro de impacto $b$. Esse parâmetro é definido como a menor distância à qual se aproximariam os regentes supondo que não existisse interação entre eles. $\mathrm{O}$ maior valor do parâmetro de impacto que conduz à ocorrência de reação é chamado de parâmetro de impacto máximo, $b_{\max }$. Colisões com parâmetro de impacto maior que $b_{\max }$ têm uma probabilidade desprezível de produzir reação.

A medida da área efetiva de colisão é a seção de choque. A seção de choque dos reagentes $\mathrm{A}+\mathrm{B}$ para formar produtos de reação deve ser função da energia translacional relativa inicial $E_{\text {tr }}$ e dos estados energéticos ro-vibracionais das espécies que interagem ${ }^{[46]}$. Assim, a seção de choque pode ser representada como $\sigma_{R}=\sigma_{R}\left(E_{\mathrm{tr}}, v, J\right)$, onde $v \mathrm{e}$ $J$ são os números quânticos vibracionais e rotacionais respectivamente. A seção de choque produto de uma combinação fixa de estados ro-vibracionais dos reagentes é referida como seção de choque específica ${ }^{[52,53]}$. Se os estados quânticos ro-vibracionais 
dos reagentes são distribuídos seguindo alguma distribuição estatística em função da temperatura, a seção de choque fica da seguinte forma:

$$
\sigma_{r}\left(E_{\mathrm{tr}}, T\right)=\sum_{v} \sum_{J} \sigma_{R}\left(E_{\mathrm{tr}}, v, J\right) P_{v}(T) P_{J}(T)
$$

onde $P_{v}$ e $P_{J}$ são, respectivamente, a distribuição de probabilidades dos estados quânticos vibracional e rotacional dos reagentes em função da temperatura $T$. Neste caso, as trajetórias quase-clássicas são calculadas combinando os estados quânticos rovibracionais dos reagentes.

Sendo $N_{T}$ o número de trajetórias calculadas e $N_{r}$ o número delas que forma reação, a seção de choque específica de reação pode ser calculada da forma ${ }^{[48]}$.

$$
\sigma_{R}=\frac{N_{r}}{N_{T}} \pi b_{\max }^{2}
$$

onde $b_{\max }$ é o parâmetro de impacto máximo.

A constante de velocidade de reação pode ser determinada multiplicando a seção de choque pela velocidade relativa $v_{r e l}$ e integrando por todo o espaço das velocidades seguindo a distribuição de Boltzmann, isto é:

$$
k(T)=\int_{0}^{\infty} \sigma\left(E_{\mathrm{tr}} ; T\right) v_{r e l} P_{B}\left(v_{r e l} ; T\right) \mathrm{d} v_{r e l}
$$

Reescrevendo esta expressão em termos da energia translacional se obtém:

$$
k(T)=\left(\frac{2}{k_{B} T}\right)^{3 / 2}\left(\frac{1}{\pi \mu}\right)^{1 / 2} \int_{0}^{\infty} E_{\mathrm{tr}} \sigma_{R}\left(E_{\mathrm{tr}} ; T\right) \exp \left(-\frac{E_{\mathrm{tr}}}{k_{B} T}\right) d E_{\mathrm{tr}},
$$

onde $\mu$ é a massa reduzida e $k_{B}$ é a constante de Boltzmann.

A seção de choque em função da energia translacional inicial é conhecida como função de excitação e contém informação importante sobre as propriedades da reação. Da forma da função de excitação pode-se determinar o tipo de colisão que se está estudando, podendo ser uma colisão sem barreira, com barreira, de tipo esfera rígida, etc. Nesta seção discutiremos um modelo de colisão que considera as reações que ocorrem sem barreira de energia potencial, devido a sua importância num dos trabalhos 
apresentado na tese. Outros modelos propostos para os outros tipos de colisões podem ser encontrados na Ref. ${ }^{[54]}$.

A colisão entre duas partículas de massas $m_{1}$ e $m_{2}$ que interagem através de forças centrais pode ser simplificado a um problema de um corpo de massa reduzida $\mu=m_{1} m_{2} /\left(m_{1}+m_{2}\right)$ sob a influência de um potencial efetivo $V_{\text {eff, dado pela soma do }}$ potencial de interação entre as partículas mais um potencial centrífugo. Neste modelo simples, se assume que os reagentes não têm estrutura interna e desde que a reação ocorre sem barreira, a energia de interação de longo alcance pode ser representada como:

$$
V(r)=-\frac{C_{n}}{r^{n}}
$$

onde $C_{n}$ e $n$ são parâmetros dependentes do tipo de interação e $r$ é a distância entre os reagentes. A interação dipolo-dipolo corresponde a valores de $n=3$, enquanto $n=4$ representaria a interação dipolo-quadrupolo e assim por diante ${ }^{[55,56]}$. Em colisões reais, as interações podem ser diferentes das interações multipolares específicas e podem até mudar ao longo da reação. Porém, neste modelo simples, esses efeitos podem ser incorporados nos valores $n$ e $C_{n}$, onde estes podem tomar valores intermediários entre os correspondentes às interações multipolares específicas.

O potencial efetivo então tem a forma:

$$
V_{\text {eff }}(r)=E_{\text {tr }} \frac{b^{2}}{r^{2}}-\frac{C_{n}}{r^{n}}
$$

onde primeiro termo da direita é o potencial centrífugo, sendo $b$ o parâmetro de impacto. Este potencial efetivo tem um máximo para $n>2 \mathrm{em} r=R_{0}$, onde:

$$
R_{0}=\left(\frac{n C_{n}}{2 E_{\mathrm{tr}} b^{2}}\right)^{1 /(n-2)}
$$

Substituindo a equação (2.10) em (2.9) obtém-se o potencial efetivo máximo da seguinte forma:

$$
V_{\mathrm{eff}}^{\max }=(n-2)\left(\frac{4 b^{2 n} E_{\mathrm{tr}}{ }^{n}}{n^{n} C_{n}^{2}}\right)^{1 / n-2} .
$$


Para cada energia translacional, o máximo do potencial efetivo é definido pelo parâmetro de impacto $b$, segundo a equação (2.11). Agora, o parâmetro de impacto máximo $\left(b_{\max }\right)$ obtém-se quando o máximo do potencial efetivo é igual à energia translacional inicial $E_{\mathrm{tr}}$. Assim, da equação (2.11), fazendo $V_{\max }^{\text {eff }}=E_{\mathrm{tr}}$ se obtém:

$$
\sigma\left(E_{\mathrm{tr}}\right)=\pi b_{\max }^{2}=n \pi(n-2)^{(2-n) / n}\left(\frac{C_{n}}{2 E_{\mathrm{tr}}}\right)^{2 / n} .
$$

Esta função é a forma funcional da seção de choque de reações de tipo captura, que deve ser ajustada aos valores de seção de choque obtidos das trajetórias quase-clássicas segundo a expressão (2.5). Substituindo (2.12) na equação da constante de velocidade de reação (2.7) e integrando, se obtém:

$$
k(T)=2 n \pi(n-2)^{(2-n) / n}\left(\frac{2}{\pi \mu}\right)^{1 / 2}\left(\frac{C_{n}}{2}\right)^{2 / n} \Gamma\left(\frac{2 n-2}{n}\right)\left(k_{B} T\right)^{(n-4) / 2 n}
$$

Mesmo quando estes resultados são obtidos do modelo simplificado apresentado, eles se ajustam muito bem a reações de tipo radical-radical ${ }^{[57]}$ e descreve muito bem as colisões de tipo captura $\mathrm{OH}+\mathrm{SO}^{[58]}$ e $\mathrm{S}+\mathrm{HO}_{2}{ }^{[59]}$, já estudadas.

\subsection{Condições iniciais}

Como foi comentado anteriormente no início da seção (2.2), nos cálculos de trajetórias quase-clássicas as condições iniciais consideram os respectivos estados quânticos rovibracionais dos reagentes. A seguir, será discutido brevemente como são geradas estas condições iniciais para reações bi-moleculares através de amostragens geradas pelo método de Monte Carlo. Este procedimento está implementado no programa de cálculo de trajetórias quase-clássicas VENUS $96^{[60]}$, que foi usado na presente tese.

A escolha de coordenadas cartesianas e momentos para cada reagente poliatômico segue o procedimento de amostragem dos modos normais ${ }^{[61]}$. Para estados quânticos rotacionais específicos de cada reagente são conhecidos o módulo do vetor momento angular e uma de suas componentes, isto é: 


$$
\begin{gathered}
j=\sqrt{J(J+1)} \hbar \\
j_{z}=K \hbar,
\end{gathered}
$$

onde $J$ e $K$ são os números quânticos rotacionais e $\hbar$ a constante de Planck. No equivalente clássico, as outras duas componentes do momento angular podem ser determinadas aleatoriamente da seguinte forma:

$$
\begin{aligned}
& j_{x}=\left(j^{2}-j_{z}^{2}\right)^{1 / 2} \sin 2 \pi R \\
& j_{y}=\left(j^{2}-j_{z}^{2}\right)^{1 / 2} \cos 2 \pi R
\end{aligned}
$$

sendo $R$ um número aleatório entre 0 e 1 .

O estado quântico vibracional de cada reagente é definido pelo número quântico $v$ em cálculos de seção de choque específicos. A energia associada a cada modo normal $i$ é $E_{i}=(v+1 / 2) \hbar \omega_{i}$. No modelo clássico, cada modo normal de vibração tem associada uma coordenada do modo normal $Q_{i}$, obtida da relação

$$
\mathbf{Q}=\mathbf{L}^{\prime} \mathbf{q} \quad \text { ou } \quad Q_{i}=\sum_{k=1}^{3 N} l_{k i} q_{k}
$$

onde $\mathbf{L}$ é a matriz formada pelos autovetores do Hessiano da energia potencial $\left(\frac{\partial^{2} U}{\partial q_{j} \partial q_{k}}\right)$. O vetor coluna $\mathbf{q}$ é formado pelos deslocamentos com relação às posições de equilíbrio das $3 N$ coordenadas do sistema, ponderadas palas massas $\left(q_{j}=m_{j}^{1 / 2} x_{j}\right)$ e num sistema de referência fixo na molécula, com origem no centro de massas. Usando as coordenadas dos modos normais, as energias, cinética e potencial do sistema, ficam da forma:

$$
T=\frac{1}{2} \sum_{i=1}^{3 N-6} \dot{Q}_{i}^{2} \quad \text { e } \quad U=\frac{1}{2} \sum_{i=1}^{3 N-6} \lambda_{i} Q_{i}^{2}
$$

sendo $N$ o número de átomos e $\lambda_{i}$ os autovalores do Hessiano $\left(\lambda_{i}=\omega_{i}^{2}\right)$. Com as energias cinética e potencial representadas desta forma, obtêm-se um conjunto de equações separáveis do tipo

$$
\frac{d^{2} Q_{i}}{d t^{2}}+\lambda_{i} Q_{i}=0
$$

com soluções: 


$$
Q_{i}=B_{i} \cos \left(\lambda_{i}^{1 / 2} t+b_{i}\right) \quad \text { ou } \quad Q_{i}=B_{i} \cos \left(\omega_{i} t+b_{i}\right)
$$

sendo $B_{i}$ e $b_{i}$ constantes. Os detalhes destes procedimentos podem ser encontrados nas Refs. ${ }^{[62,63]}$.

Fazendo a equivalência entre a energia do estado quântico vibracional e o resultado clássico obtém-se a seguinte relação para cada modo normal:

$$
E_{i}=\frac{P_{i}^{2}+\omega_{i}^{2} Q_{i}^{2}}{2}
$$

Assim, as coordenadas e momentos iniciais dos modos normais são geradas aleatoriamente da seguinte forma:

$$
Q_{i}=\left[\left(2 E_{i}\right)^{1 / 2} / \omega_{i}\right] \cos \left(2 \pi R_{i}\right) \quad \text { e } \quad P_{i}=-\left(2 E_{i}\right)^{1 / 2} \operatorname{sen}\left(2 \pi R_{i}\right)
$$

onde $R_{i}$ são números aleatórios.

As coordenadas normais e momentos $\mathbf{Q}$ e $\mathbf{P}$, assim como o momento angular rotacional são transformados às coordenadas cartesianas e momentos no sistema de referência do centro de massa através do seguinte procedimento.

- As coordenadas dos modos normais e momentos $\mathbf{Q}$ e $\mathbf{P}$ são transformadas para as coordenadas cartesianas da seguinte forma ${ }^{[62]}$ :

$$
\begin{aligned}
& \mathbf{x}=\mathbf{x}_{\mathbf{0}}+\mathbf{M}^{-1 / 2} \mathbf{L Q} \\
& \mathbf{p}=\mathbf{M}^{1 / 2} \mathbf{L P}
\end{aligned}
$$

sendo $\mathbf{x}_{\mathbf{0}}$ o vetor das coordenadas de equilíbrio e $\mathbf{M}$ uma matriz diagonal cujo os elementos são as massas atômicas, lembre-se que as coordenadas q são ponderadas pelas massas.

- Desde que os modos normais são calculados aproximadamente através de deslocamentos finitos ${ }^{[62]}$, pode surgir algum momento angular espúrio após estas transformações ${ }^{[64,65]}$. Esse momento angular é encontrado da seguinte forma. Até agora o sistema de referência está fixado na molécula, girando e se transladando junto com ela. Qualquer momento angular que apareça nesse sistema de referência vai ser espúrio e é determinado da seguinte forma: 


$$
\mathbf{j}_{\mathbf{s}}=\sum_{\mathbf{i}=1}^{\mathrm{N}} \mathbf{r}_{\mathbf{i}} \times \mathbf{m}_{\mathbf{i}} \dot{\mathbf{r}}_{\mathbf{i}}
$$

Assim, o momento angular adicionado à molécula vai ser o momento angular determinado anteriormente (2.15) tirando o momento angular espúrio:

$$
\mathbf{j}=\mathbf{j}_{\mathbf{0}}-\mathbf{j}_{\mathbf{s}}
$$

A velocidade colocada em cada átomo proveniente da rotação vai ser então $\boldsymbol{\omega} \times \mathbf{r}_{\mathbf{i}}$, onde:

$$
\omega=\mathbf{I}^{-1} \mathbf{j},
$$

e $\mathbf{I}^{-1}$ o inverso do tensor momento de inércia ${ }^{[51]}$.

- A energia interna $E$ colocada na molécula através das coordenadas cartesianas e momentos iniciais deve ser igual à energia interna desejada $E^{0}$. Se esta igualdade não é cumprida dentro de determinado erro, as coordenadas cartesianas e momentos são escalados segundo as relações:

$$
\begin{aligned}
& x_{i}^{\prime}=x_{i}^{0}+\left(x_{i}-x_{i}^{0}\right)\left(E^{0} / E\right)^{1 / 2} \\
& p_{i}^{\prime}=p_{i}\left(E^{0} / E\right)^{1 / 2}
\end{aligned}
$$

Qualquer energia translacional espúria que apareça do centro de massa é subtraída da energia da molécula. Repete-se então o ciclo a partir do segundo passo.

Uma vez concluído este procedimento, as coordenadas cartesianas selecionadas para uma das moléculas (A) são rotacionadas aleatoriamente em relação ao sistema de coordenadas fixo externo ao sistema, a fim de definir uma orientação aleatória. Isso é feito através dos ângulos de Euler ${ }^{[66]}$, ou seja:

$$
\mathbf{x}=\mathbf{R}(\theta, \phi, \chi) \mathbf{x}_{\mathbf{0}} \quad \mathrm{e} \quad \dot{\mathbf{x}}=\mathbf{R}(\theta, \phi, \chi) \dot{\mathbf{x}}_{\mathbf{0}}
$$

onde $\mathbf{x}_{0} \mathrm{e} \quad \dot{\mathbf{x}}_{\mathbf{0}}$ são vetores formados pelas coordenadas cartesianas e velocidades selecionadas no procedimento mostrado anteriormente e $\mathbf{R}(\theta, \phi, \chi)$ é a matrix de rotação de Euler. Os ângulos $\theta, \phi, \chi$ são selecionados aleatoriamente segundo as relações:

$$
\cos \theta=2 R_{1}-1, \quad \phi=2 \pi R_{2}, \quad \chi=2 \pi R_{3},
$$


sendo $R_{1}, R_{2}$ e $R_{3}$ três números aleatórios diferentes.

Desde que o reagente poliatômico A tem uma orientação aleatória no sistema de referência fixo, o centro de massa do reagente $\mathrm{B}$ pode ser colocado no plano $y z$ sem perder generalidade. Dessa forma, as coordenadas de B são:

$$
x=0, \quad y=b, \quad z=\left(s^{2}-b^{2}\right)^{1 / 2}
$$

onde $b$ é o parâmetro de impacto definido aleatoriamente para cada trajetória, tal que $b<b_{\max }$, e $s$ é a separação inicial entre os centros de massa dos reagentes.

A velocidade relativa $v_{\text {rel }}$ entre A e B é adicionada ao longo do eixo $z$, com a restrição de que o centro de massa entre os reagentes fique fixo. Assim, as componentes da $v_{\text {rel }}$ são zero nos eixos $x$ e $y$, igual a $\left[m_{\mathrm{A}} /\left(m_{\mathrm{A}}+m_{\mathrm{B}}\right)\right] v_{\text {rel }}$ na componente $z$ de cada átomo do reagente $\mathrm{B}$ e igual a $-\left[m_{\mathrm{B}} /\left(m_{\mathrm{A}}+m_{\mathrm{B}}\right)\right] v_{\text {rel }}$ na componente $z$ de cada átomo do reagente A.

Desta forma, as condições iniciais dos reagentes são determinadas, sendo reproduzidos os estados quânticos ro-vibracionais dos reagentes e a velocidade de translação estudada. 
52 Superfícies de energia potencial e método de trajetórias quase-clássicas 


\section{Capítulo 3}

\section{Simulação clássica de Monte Carlo}

Neste capítulo vamos considerar os elementos teóricos da metodologia sequencial QM/MM, usada nesta tese para estudar o efeito de solvente em propriedades espectroscópicas de sistemas moléculas. Nesta metodologia são usados conceitos de mecânica molecular e física estatística, combinados de forma sequencial com cálculos de mecânica quântica.

De forma geral, fazendo simulações clássicas baseadas no método Monte Carlo

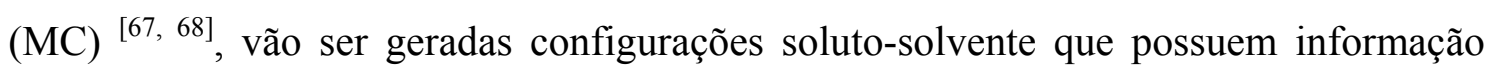
estatística relevante do sistema e reproduzam as propriedades macroscópicas do líquido. Essas configurações serão usadas nos cálculos de mecânica quântica para determinar os valores médios das propriedades quânticas de interesse no sistema molecular.

Iniciaremos o presente capítulo descrevendo o processo Markoviano ${ }^{[68]}$, usado na seleção de configurações representativas do espaço configuracional do líquido. Posteriormente, discutiremos como é mapeado o espaço configuracional usando a amostragem de Metropolis ${ }^{[67]}$, de forma que uma amostragem relevante do sistema possa oferecer toda a informação estatística do sistema em equilíbrio termodinâmico. Posteriormente serão discutidos conceitos como o potencial interatômico usado nos cálculos clássicos, polarização eletrônica do soluto, função de autocorrelação da energia e função de distribuição radial. 


\subsection{Processo Markoviano e amostragem de Metropolis}

O método de Monte Carlo ${ }^{[67]}$ é conhecido por ser um método de resolução de integrais usando uma amostragem aleatória do sistema quando o espaço de integração é muito grande. Por exemplo, em nosso caso estaríamos interessados em calcular integrais do tipo:

$$
\langle f\rangle=\frac{\int_{\{\Gamma\}} f(\Gamma) P(\Gamma) \mathrm{d} \Gamma}{\int_{\{\Gamma\}} P(\Gamma) \mathrm{d} \Gamma},
$$

que representa a média de uma magnitude macroscópica dos líquidos $(f)$. Essa média seria determinada explorando todo o espaço de configurações do líquido (um espaço infinito) onde $P(\Gamma)$ é a probabilidade de encontrar o valor $f(\Gamma)$ nesse espaço. $\mathrm{O}$ processo para obter configurações aleatórias representativas do sistema é conhecido como processo Markoviano ${ }^{[68]}$. Nesses processos as configurações obtêm-se de forma sequencial seguindo as seguintes relações:

$$
\begin{array}{ll}
\pi_{i j}=1, & \text { se } U_{j} \leq U_{i} \text { para } i \neq j \\
\pi_{i j}=e^{-\beta\left\lfloor\Delta U+p \Delta V+N \ln \left(\mathrm{V}_{j} / V_{i}\right)\right\rfloor}, & \text { se } U_{j}>U_{i} \text { para } i \neq j \\
\pi_{i j}=1-\sum_{i \neq j} \pi_{i j}, & \text { se } i=j,
\end{array}
$$

onde $\pi_{i j}$ é a probabilidade de transição entre as configurações $\Gamma_{i}$ e $\Gamma_{j}$. A exponencial da equação (3.3) é o peso de Boltzmann no ensemble NPT e $U_{k}$ indica a energia da configuração $\Gamma_{k}$.

O processo Markoviano funciona da seguinte maneira. Partindo de uma configuração inicial $\Gamma_{i}$, é gerada aleatoriamente uma nova configuração $\Gamma_{j}$. Se a diferença $\Delta U=U_{j}-U_{i}$, é menor que zero, a nova configuração é aceita. No caso contrário, quando $\Delta U$ é maior que zero, se calcula o peso de Boltzmann (3.3) e é 
gerado um número aleatório entre 0 e 1 , se o número aleatório é menor que o peso de Boltzmann se aceita a nova configuração $\Gamma_{j}$, caso contrário, ela é descartada e $\Gamma_{i}$ é mantida. Gera-se então uma outra configuração aleatória $\Gamma_{j}$ para realizar novamente o processo.

Um conjunto de configurações $\{\Gamma\}$, obtido do processo descrito anteriormente, contém suficiente informação estatística para descrever as propriedades de equilíbrio termodinâmico do sistema estudado. Portanto, a proposta de Metropolis ${ }^{[67]}$ foi resolver as integrais do tipo (3.1) reduzindo o espaço de integração ao conjunto $\{\Gamma\}$. Levando em consideração que a quantidade de configurações agora é um número finito $l$, podemos trocar a integral de (3.1) por uma somatória. Além disso, sabendo que cada configuração obteve-se considerando o peso de Boltzmann, não é preciso multiplicar novamente pelo peso $P(\Gamma)$ e a expressão para determinar os valores médios será:

$$
\langle f\rangle=\frac{1}{l} \sum_{l} f_{l}
$$

com o correspondente erro estatístico:

$$
\sigma=\sqrt{\frac{\left\langle A^{2}\right\rangle-\langle A\rangle^{2}}{l-1}}
$$

Em outras palavras, os valores médios de magnitudes macroscópicas dos líquidos em equilíbrio termodinâmico vão ser a média aritmética dos valores medidos em cada configuração aceita $\left(f_{l}=f\left(\Gamma_{l}\right)\right)$, uma vez que o peso de Boltzmann já foi considerado no processo Markoviano para a seleção do conjunto de configurações $\{\Gamma\}$.

Essa metodologia está implementada no código DICE ${ }^{[69]}$, que foi usado no presente trabalho nas simulações clássicas dos líquidos estudados. Só resta comentar alguns detalhes importantes como, por exemplo, que a energia $U_{k}$ da configuração $\Gamma_{k}$ é determinada classicamente, considerando interações coulombianas e o potencial de Lennard-Jones, como será explicado na próxima seção. Também vale notar que a forma para gerar uma nova configuração, partindo da configuração atual, é feita transladando o centro de massa de uma molécula uma distância $\delta \mathbf{r}$ e rotacionando ela num ângulo que varia entre +15 e $-15^{\circ}$ em torno de um de seus eixos. Tanto a direção de translação 
como a seleção do eixo de rotação são escolhidos aleatoriamente. Um passo de MC é definido quando esse processo é feito em cada uma das moléculas do líquido.

\subsection{Potencial intermolecular}

Nas simulações clássicas dos líquidos realizadas neste trabalho, as moléculas, tanto do líquido quanto do soluto, são consideradas rígidas. Essa consideração faz com que as interações intramoleculares (stretching, bending, etc.) não sejam consideradas no hamiltoniano clássico e, unicamente, as interações intermoleculares são levadas em conta. O potencial de interação clássico intermolecular entre duas moléculas é então:

$$
U=\sum_{i=1}^{N_{a}} \sum_{j=1}^{N_{b}} U_{i j}
$$

onde $N_{a}$ e $N_{b}$ são a quantidade de sítios ativos das moléculas $a$ e $b$ respectivamente. $U_{i j}$ é o potencial de Lennard-Jones (LJ) acrescido do potencial coulombiano:

$$
U_{i j}=4 \varepsilon_{i j}\left[\left(\frac{\sigma_{i j}}{r_{i j}}\right)^{12}-\left(\frac{\sigma_{i j}}{r_{i j}}\right)^{6}\right]+\frac{q_{i} q_{j}}{r_{i j}}
$$

Os parâmetros do potencial de LJ dependem dos tipos de átomos que estão interagindo e são determinados da seguinte forma: $\sigma_{i j}=\sqrt{\sigma_{i} \sigma_{j}}$ e $\varepsilon_{i j}=\sqrt{\varepsilon_{i} \varepsilon_{j}}$, onde os valores $\sigma_{k}$ e $\varepsilon_{k}$ vão depender somente das características de um dos átomos e do entorno químico onde ele se encontra. As cargas $q_{k}$ são as cargas de cada sítio de interação das moléculas. Os parâmetros de LJ e os valores das cargas usados nas moléculas dos líquidos considerados nesta tese, vão ser obtidos de campos de força reportados na literatura que são otimizados para simulações de líquidos.

No soluto, esses parâmetros de LJ são obtidos dos campos de força OPLS (Optimized Parameters for Liquid Simulations), mas os valores das cargas são obtidos num procedimento iterativo de polarização eletrônica, que garante o equilíbrio eletrostático entre o soluto e o solvente. Esse procedimento iterativo é discutido na seção (3.4). 


\subsection{Função de autocorrelação da energia}

Na seção (3.1) foi mostrado como nas simulações de MC, um número determinado de configurações pode conter toda a informação estatística relevante do sistema. Para complementar toda a análise realizada nessa seção é importante incluir o conceito de função de autocorrelação. Numa simulação de MC obtém-se uma grande quantidade de configurações, da ordem de $10^{9}$ e muitas delas carregam informações estatísticas muito parecidas. A fim de eliminar configurações que não oferecem informação nova do sistema é usada a função de autocorrelação da energia, definida da seguinte forma:

$$
C(i)=\frac{\left\langle\delta E_{n} \delta E_{n+i}\right\rangle}{\left\langle\delta E^{2}\right\rangle}=\frac{\sum_{n}\left(E_{n}-\langle E\rangle\right)\left(E_{n+i}-\langle E\rangle\right)}{\sum_{n}\left(E_{n}-\langle E\rangle\right)^{2}}
$$

Nesta expressão, $E_{n}$ é a energia da configuração $n$ e $E_{n+i}$ a energia da configuração gerada $i$ passos de MC depois. Assim, pode-se notar que configurações separadas por um número pequeno de passos de MC ( $i$ pequeno) possuem uma correlação da ordem da unidade e não teríamos informação estatística nova ao incluir as duas na amostragem representativa do sistema. Obviamente, só teríamos configurações completamente descorrelacionadas no caso $i=\infty$.

Em processos Markovianos de sistemas líquidos, é sabido que a função de

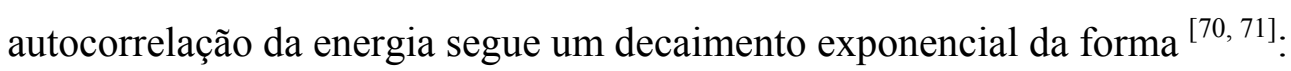

$$
C(i)=\sum_{n} C_{n} e^{-i / \tau_{n}}
$$

Porém, nos estudos realizados no nosso grupo de trabalho ${ }^{[72]}$ tem sido observado que um decaimento exponencial de segunda ordem descreve muito bem a função de autocorrelação da energia:

$$
C(i)=c_{1} e^{-i / \tau_{1}}+c_{2} e^{-i / \tau_{2}}
$$

onde $c_{i}$ e $\tau_{i}$ são constantes de ajuste. Na prática, duas configurações são consideradas descorrelacionadas se estiverem separadas por um intervalo $i=2 \tau$, onde $\tau$ obtém-se da seguinte integral: 


$$
\tau=\int_{0}^{\infty} C(i) \mathrm{d} i=c_{1} \tau_{1}+c_{2} \tau_{2} .
$$

Em geral, configurações separadas por intervalos maiores que $2 \tau$ têm uma correlação menor do que $15 \%$.

Podemos concluir que de todo o conjunto de configurações geradas numa simulação de $\mathrm{MC}$, uma convergência rápida pode ser obtida se são selecionadas somente aquelas separadas por $2 \tau$.

\subsection{Polarização eletrônica}

Quando uma molécula é envolvida por um solvente, a sua nuvem eletrônica é modificada, sendo influenciada pelas interações eletrostática com as moléculas do meio. Acarretando assim, mudanças nos momentos eletrostáticos do soluto. Esse efeito de polarização eletrônica do soluto é muito importante ${ }^{[73-77]}$ e é levado em consideração nos trabalhos apresentados nesta tese através de um procedimento iterativo de simulações clássicas de $\mathrm{MC}^{[78]}$.

Para alcançar o equilíbrio eletrostático soluto-solvente, é feito um cálculo inicial das cargas do soluto (por exemplo, em fase gasosa) no estado eletrônico de interesse, usando o mapeamento eletrostático CHELPG ${ }^{[79]}$ (Charges from Electrostatic Potentials using a Grid based method). Essas cargas são inicialmente usadas no potencial clássico da simulação de MC. Após a primeira simulação de MC, as configurações solutosolvente estatisticamente descorrelacionadas ${ }^{[70]}$ são usadas para determinar novamente as cargas e momento de dipolo médios do soluto. Isso é feito num cálculo que considera o soluto envolvido no campo eletrostático produzido pelas cargas do solvente (ASEC, Average Solvent Eletrostatic Configuration) ${ }^{[80]}$, onde somente uma configuração média é gerada de tal forma que reproduz o mesmo valor médio que se obteria ao utilizar todas as configurações. As cargas calculadas são usadas numa nova simulação de $\mathrm{MC}$ e o processo é continuado iterativamente até alcançar a convergência do momento de dipolo, que indica que foi alcançado o equilíbrio eletrostático soluto-solvente. Nesse ponto, obtêm-se as configurações soluto-solvente descorrelacionadas a serem usadas nos cálculos de mecânica quântica. 


\subsection{Função de distribuição radial}

A função de distribuição radial (RDF) é muito usada para descrever camadas de solvatação ao redor dos solutos. Ela determina a quantidade de moléculas separadas por uma distância entre $r$ e $r+d r$ do soluto, tomando como referência a quantidade que existiriam num gás ideal de igual densidade que o líquido. Nas simulações é usada a seguinte expressão para determinar a RDF:

$$
G_{i j}(r)=\frac{n_{i j}(r, r+d r)}{n_{\text {ideal }}(r, r+d r)}=\frac{n_{i j}(r, r+d r)}{(4 / 3) \pi \rho\left[(r+d r)^{3}-r^{3}\right]},
$$

onde $n_{i j}$ é o número de moléculas do líquido separadas por uma distância entre $r$ e $r+d r$, enquanto $n_{\text {ideal }}$ é o número de moléculas correspondentes no gás ideal, calculado segundo indica o segundo denominador.

Quando o soluto é uma molécula com simetria aproximadamente esférica, pode-se analisar a RDF das moléculas de solvente com relação ao soluto levando em consideração as distâncias entre centros de massa. Nesse caso, as moléculas de solventes possuem uma distribuição em forma de camadas numa simetria quase esférica ao redor do soluto. No caso de moléculas de soluto alongadas a análise entre centros de massa carece de sentido. Portanto, é preciso usar a função de distribuição de mínima

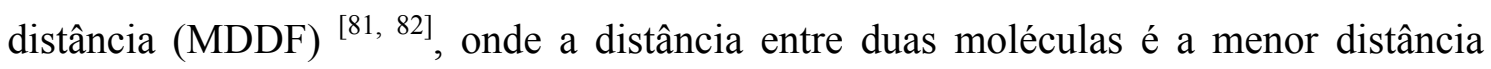
entre os átomos de uma molécula e os átomos da outra molécula. Ao considerar a função de distribuição dessa forma, as camadas de solvatação possuem uma distribuição similar à simetria do soluto.

Para determinar o número de moléculas de solvente, nas camadas de solvatação, deve-se integrar a função RDF, ficando:

$$
N_{m o l}=4 \pi \rho \int_{r_{1}}^{r_{2}} G(r) r^{2} \mathrm{~d} r .
$$

O número de moléculas que forma as diferentes camadas é importante para selecionar a quantidade de moléculas explícitas a serem colocadas nos cálculos de mecânica quântica. 
60 Simulação clássica de Monte Carlo 
II. Sistemas estudados 


\section{Capítulo 4}

\section{Estudo de trajetórias quase-clássicas da reação OH+SO: Influência da}

\section{energia rotacional}

O enxofre é considerado um dos maiores poluentes atmosféricos, com grandes implicações nas condições ambientais. Este elemento participa do ciclo de formação da chuva ácida, contribui na poluição do ar e nas mudanças climatológicas globais ${ }^{[83-85]}$. Embora uma parte significativa das moléculas que contêm enxofre reagem com a água na troposfera e eventualmente precipitam-se como aerossóis, alguns destes compostos (como o COS) podem alcançar a estratosfera, onde acabam influenciando no ciclo do ozônio ${ }^{[83]}$. Particularmente, o sistema molecular $\mathrm{HSO}_{2}$ tem um papel relevante nestes processos climatológicos, além de formar parte de processos de combustão ${ }^{[86-97]}$. Portanto, na busca de um melhor entendimento desses processos, muitos trabalhos teóricos e experimentais têm sido realizados para estudar este sistema molecular e as reações químicas associadas, como as mostradas na Tabela 4.1 (tomada da Ref. ${ }^{[98]}$ ).

Entre as reações de interesse no sistema molecular $\mathrm{HSO}_{2}$ (tabela 4.1) encontra-se a oxidação do enxofre. Acredita-se que esta reação seja controlada pelos radicais hydroperoxyl e hydroxyl ${ }^{[83]}$, que são radicais livres abundantes na atmosfera ${ }^{[99,100]}$. Por esta razão, a reação

$$
\mathrm{OH}+\mathrm{SO} \rightarrow \mathrm{H}+\mathrm{SO}_{2}
$$


tem sido amplamente estudada tanto teórica ${ }^{[93,101-103]}$ quanto experimentalmente ${ }^{[104-}$ ${ }^{106]}$. Já foi publicado um trabalho ${ }^{[58]}$ onde se realizou um estudo teórico de dinâmica quase-clássica da reação (4.1), proporcionando detalhes dos mecanismos de colisão e da constante de reação. Nesse trabalho os estados iniciais ro-vibracionais dos reagentes foram mantidos nos correspondentes estados fundamentais.

Por outra parte, vários trabalhos têm reportado a influência da energia rotacional do $\mathrm{OH}$ em reações com átomos. No trabalho de Jorfi et al. ${ }^{[107]}$ obteve-se um aumento da constante de reação em $\mathrm{OH}+\mathrm{O} \rightarrow \mathrm{H}+\mathrm{O}_{2}$ quando se deposita energia rotacional no radical OH. Também têm sido observados efeitos significativos na seção de choque e constante de reação na colisão $\mathrm{N}+\mathrm{OH}{ }^{[108]}$ quando o número quântico rotacional de $\mathrm{OH}\left(j_{\mathrm{OH}}\right)$ é aumentado. Porém, poucas mudanças têm sido observadas na reação $\mathrm{C}+\mathrm{OH}$ quando se incrementa a energia rotacional de $\mathrm{OH}^{[109]}$.

O principal objetivo do presente trabalho é fazer um estudo teórico detalhado da reação

$$
\mathrm{OH}\left(v^{\prime}=j_{\mathrm{OH}}\right)+\mathrm{SO}\left(v^{\prime \prime}=j_{\mathrm{SO}}\right) \rightarrow \mathrm{H}+\mathrm{SO}_{2},
$$

a fim de investigar o efeito da energia rotacional dos reagentes $\left(v^{\prime}\right.$ e $\left.v^{\prime \prime}\right)$ sobre a reatividade. Como foi discutido no capítulo (2), uma forma viável de estudar as reações químicas teoricamente consiste em representar analiticamente a superfície de energia potencial global do sistema molecular de interesse e estudar a dinâmica das reações usando a SEP. A SEP global do sistema molecular $\mathrm{HSO}_{2}$ no estado eletrônico fundamental já foi construída ${ }^{[98]}$ e utilizada no estudo teórico de várias reações

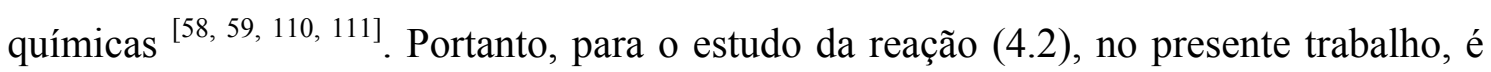
usada esta SEP e o método de trajetórias quase-clássicas ${ }^{[38]}$.

Este trabalho é organizado da seguinte forma: na próxima seção é apresentada a descrição da SEP. Na seção (4.2) é mostrada a metodologia usada nos cálculos de trajetórias quase-clássicas e na seção (4.3) são discutidos os resultados obtidos. Finalmente são feitas as conclusões do trabalho. 
Tabela 4.1 Processos exotérmicos que ocorrem na superfície de energia potencial do $\mathrm{HSO}_{2}$ no estado fundamental. Os valores energéticos são reproduzidos na SEP DMBE.

\begin{tabular}{ccc}
\hline \multicolumn{1}{c}{ Processo } & $\begin{array}{c}\text { Exotermicidade } \\
\text { clássica }(\mathrm{kcal} / \mathrm{mol})\end{array}$ & Ref. \\
\hline $\mathrm{HS}+\mathrm{O} 2 \rightarrow \mathrm{H}+\mathrm{SO} 2$ & 24.1 & {$[112-114]$} \\
$\mathrm{HS}+\mathrm{O} 2 \rightarrow \mathrm{OH}+\mathrm{SO}$ & 51.7 & {$[113-115]$} \\
$\mathrm{OH}+\mathrm{SO} \rightarrow \mathrm{H}+\mathrm{SO} 2$ & 27.6 & {$[112,114,115]$} \\
$\mathrm{H}+\mathrm{SOO} \rightarrow \mathrm{S}+\mathrm{HO} 2$ & 30.6 & {$[115,116]$} \\
$\mathrm{H}+\mathrm{SOO} \rightarrow \mathrm{HS}+\mathrm{O} 2$ & 63.3 & {$[112,114,115]$} \\
$\mathrm{H}+\mathrm{SOO} \rightarrow \mathrm{OH}+\mathrm{SO}$ & 87.5 & {$[115]$} \\
$\mathrm{H}+\mathrm{SOO} \rightarrow \mathrm{H}+\mathrm{SO} 2$ & 115.0 & {$[112,113]$} \\
$\mathrm{O}+\mathrm{HSO} \rightarrow \mathrm{HS}+\mathrm{O} 2$ & 21.7 & {$[112,114]$} \\
$\mathrm{O}+\mathrm{HSO} \rightarrow \mathrm{OH}+\mathrm{SO}$ & 45.8 & {$[112,115]$} \\
$\mathrm{O}+\mathrm{HSO} \rightarrow \mathrm{H}+\mathrm{SO} 2$ & 73.4 & {$[112,116]$} \\
$\mathrm{S}+\mathrm{HO} 2 \rightarrow \mathrm{O}+\mathrm{HSO}$ & 11.0 & {$[112,114,116]$} \\
$\mathrm{S}+\mathrm{HO} 2 \rightarrow \mathrm{HS}+\mathrm{O} 2$ & 32.8 & {$[115,116]$} \\
$\mathrm{S}+\mathrm{HO} 2 \rightarrow \mathrm{OH}+\mathrm{SO}$ & 56.9 & \\
$\mathrm{~S}+\mathrm{HO} 2 \rightarrow \mathrm{H}+\mathrm{SO} 2$ & 84.4 &
\end{tabular}

\subsection{Superfície de energia potencial}

A SEP hexa-dimensional do sistema $\mathrm{HSO}_{2}$ no estado fundamental ${ }^{[98]}$ foi representada usando a metodologia de expansão dupla de muitos corpos comentada na seção (2.1). Essa metodologia usa funções DMBE anteriormente reportadas para os fragmentos diatômicos e triatômicos deste sistema molecular, acrescido de um termo energético de quatro corpos para ajustar a SEP a cálculos ab initio do sistema tetratômico, nesse caso, a cálculos no nível CASPT2/aug-cc-pVXZ $(X=\mathrm{D}, \mathrm{T})$.

Na figura 4.1 é mostrado um gráfico de contorno da reação (4.1) de acordo com a SEP DMBE ${ }^{[98]}$. O isômero HOSO é o mínimo global deste sistema, com o átomo de hidrogênio ligado ao oxigênio e com energia de $-71.8 \mathrm{kcal} \mathrm{mol}^{-1}$ em relação ao limite de dissociação $\mathrm{OH}+\mathrm{SO}$. Deve-se notar a ausência de barreira para a formação desse isômero. Na figura 4.2 (tomada da Ref. ${ }^{[98]}$ ) é mostrado o diagrama energético global da SEP DMBE do $\mathrm{HSO}_{2}$. 


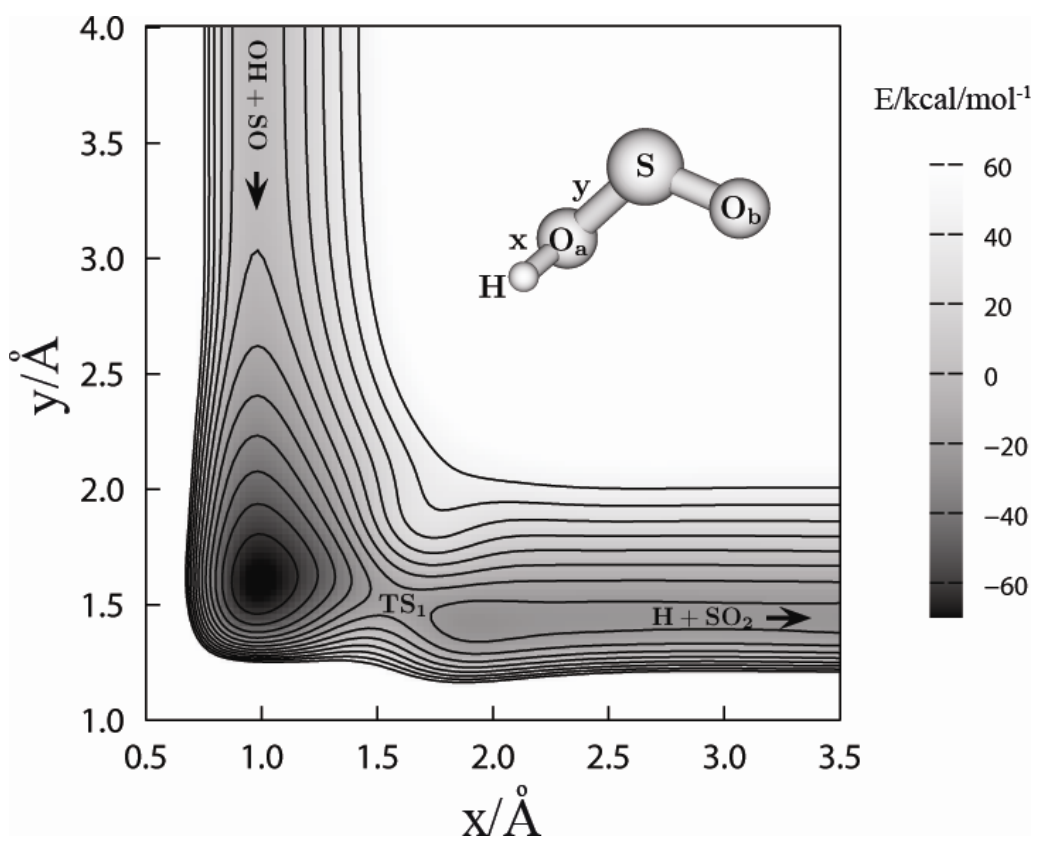

Figura 4.1. Gráfico de contorno da reação estudada segundo a SEP DMBE. Os valores energéticos têm como referência o limite de dissociação $\mathrm{OH}+\mathrm{SO}$. Os contornos começam em $70 \mathrm{kcal} \mathrm{mol}^{-1}$, sendo igualmente espaçados por $10 \mathrm{kcal} \mathrm{mol}^{-1}$. $\mathrm{O}$ eixo " $\mathrm{x}$ " representa a distância $\mathrm{O}_{\mathrm{a}}-\mathrm{H}$, o eixo "y" representa a distância $\mathrm{S}-\mathrm{O}_{\mathrm{a}}$, enquanto que as quatro variáveis restantes são parcialmente relaxadas ao redor da configuração de equilíbrio do mínimo global HOSO.

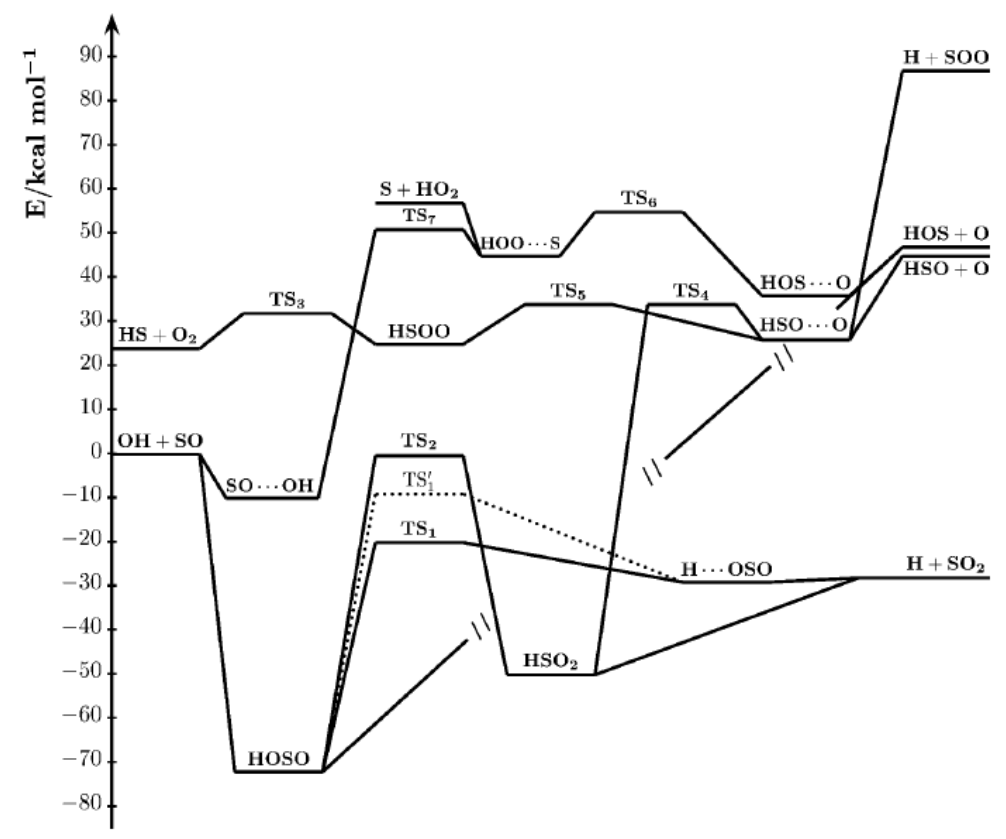

Figura 4.2. Diagrama energético da superfície de energia potencial global do $\mathrm{HSO}_{2}$. Tomado da Ref. ${ }^{[98]}$ 


\subsection{Procedimentos computacionais}

Os cálculos de trajetórias quase-clássicas, no estudo da reação (4.2) foram realizados usando uma versão adaptada do código VENUS $96^{[60]}$, onde a SEP DMBE do $\mathrm{HSO}_{2}$ foi inserida como potencial de interação. Essa metodologia já foi usada para estudar o efeito das excitações vibracionais dos reagentes, em reações como $\mathrm{HS}+\mathrm{O}_{2}{ }^{[117,118]}$, e no estudo do efeito da energia rotacional do $\mathrm{OH}$ nas reações $(\mathrm{C}, \mathrm{O}, \mathrm{N})+\mathrm{OH}^{[107-109]}$.

Cada trajetória calculada pode conduzir a 14 canais de reação diferentes, como é indicado na tabela 4.2, isto desconsiderando os vários isômeros triatômicos possíveis. $\mathrm{O}$ primeiro canal corresponde aos reagentes.

Tabela 4.2 Canais de reação. A nomenclatura das distâncias interatômicas é a seguinte: $\mathrm{R}_{1} \rightarrow \mathrm{SO}_{\mathrm{a}}, \mathrm{R}_{2} \rightarrow \mathrm{SO}_{\mathrm{b}}, \mathrm{R}_{3} \rightarrow \mathrm{SH}, \mathrm{R}_{4} \rightarrow \mathrm{O}_{\mathrm{a}} \mathrm{O}_{\mathrm{b}}, \mathrm{R}_{5} \rightarrow \mathrm{O}_{\mathrm{a}} \mathrm{H}, \mathrm{R}_{6} \rightarrow \mathrm{O}_{\mathrm{b}} \mathrm{H}$.

\begin{tabular}{cccccccc}
\hline Canal & Produtos & $\mathrm{R}_{1} / \AA$ & $\mathrm{R}_{2} / \AA$ & $\mathrm{R}_{3} / \AA$ & $\mathrm{R}_{4} / \AA$ & $\mathrm{R}_{5} / \AA$ & $\mathrm{R}_{6} / \AA$ \\
\hline 1 & $\mathrm{O}_{\mathrm{a}} \mathrm{H}+\mathrm{SO}_{\mathrm{b}}$ & $\infty$ & 1.481 & $\infty$ & $\infty$ & 0.971 & $\infty$ \\
2 & $\mathrm{O}_{\mathrm{b}} \mathrm{H}+\mathrm{SO}_{\mathrm{a}}$ & 1.481 & $\infty$ & $\infty$ & $\infty$ & $\infty$ & 0.971 \\
3 & $\mathrm{HS}+\mathrm{O}_{2}$ & $\infty$ & $\infty$ & 1.340 & 1.208 & $\infty$ & $\infty$ \\
4 & $\mathrm{H}+\mathrm{SO}_{2}$ & 1.431 & 1.431 & $\infty$ & 2.470 & $\infty$ & $\infty$ \\
5 & $\mathrm{O}_{\mathrm{a}}+\mathrm{HSO}_{\mathrm{b}}$ & $\infty$ & 1.512 & 1.386 & $\infty$ & $\infty$ & 2.374 \\
6 & $\mathrm{O}_{\mathrm{b}}+\mathrm{HSO}_{\mathrm{a}}$ & 1.512 & $\infty$ & 1.386 & $\infty$ & 2.374 & $\infty$ \\
7 & $\mathrm{~S}+\mathrm{HO}_{2}$ & $\infty$ & $\infty$ & $\infty$ & 1.331 & 0.971 & 1.831 \\
8 & $\mathrm{O}_{\mathrm{a}}+\mathrm{H}+\mathrm{SO}_{\mathrm{b}}$ & $\infty$ & 1.481 & $\infty$ & $\infty$ & $\infty$ & $\infty$ \\
9 & $\mathrm{~S}+\mathrm{O}_{\mathrm{b}}+\mathrm{HO}_{\mathrm{a}}$ & $\infty$ & $\infty$ & $\infty$ & $\infty$ & 0.971 & $\infty$ \\
10 & $\mathrm{O}_{\mathrm{b}}+\mathrm{H}+\mathrm{SO}_{\mathrm{a}}$ & 1.481 & $\infty$ & $\infty$ & $\infty$ & $\infty$ & $\infty$ \\
11 & $\mathrm{~S}+\mathrm{O}_{\mathrm{a}}+\mathrm{HO}_{\mathrm{b}}$ & $\infty$ & $\infty$ & $\infty$ & $\infty$ & $\infty$ & 0.971 \\
12 & $\mathrm{~S}+\mathrm{H}+\mathrm{O}_{2}$ & $\infty$ & $\infty$ & $\infty$ & 1.208 & $\infty$ & $\infty$ \\
13 & $\mathrm{O}_{\mathrm{a}}+\mathrm{O}_{\mathrm{b}}+\mathrm{HS}$ & $\infty$ & $\infty$ & 1.340 & $\infty$ & $\infty$ & $\infty$ \\
14 & $\mathrm{~S}+\mathrm{O}_{\mathrm{a}}+\mathrm{O}_{\mathrm{b}}+\mathrm{H}$ & $\infty$ & $\infty$ & $\infty$ & $\infty$ & $\infty$ & $\infty$ \\
\hline
\end{tabular}

O efeito da energia rotacional sobre a reatividade da reação (4.2) foi considerado usando vários níveis quânticos rotacionais como condições iniciais dos reagentes nos cálculos das trajetórias. Para o radical $\mathrm{OH}$ foram usadas as energias rotacionais correspondentes aos números quânticos $1 \leq j_{\mathrm{OH}} \leq 12$, enquanto para o diátomo $\mathrm{SO}$ foram selecionados números quânticos múltiplos de 5 no intervalo $1 \leq j_{\text {so }} \leq 60$. O estado rotacional fundamental do $\mathrm{OH}$ é representado por $j=1$, pois o estado eletrônico fundamental é da forma $\mathrm{OH}\left({ }^{2} \Pi\right)$, que tem momento angular intrínseco. Foram realizados cálculos de trajetórias onde os dois reagentes são excitados rotacionalmente, para comparação com os resultados experimentais. As energias vibracionais dos reagentes foram mantidas nos correspondentes estados quânticos fundamentais $v^{\prime}=0 \mathrm{e}$ 
$v^{\prime \prime}=0$ em todos os cálculos. A energia rotacional é considerada usando o modelo do rotor rígido quântico, já que são levados em conta relativamente poucos estados quânticos rotacionais e as moléculas estão no estado vibracional fundamental.

O intervalo considerado para a integração numérica no cálculo das trajetórias foi selecionado de forma que a energia total fosse conservada. Assim, o passo de $2.5 \times 10^{-16}$ s garante uma conservação da energia total com uma precisão melhor que uma parte em $10^{3}$.

As moléculas reagentes são inicialmente separadas por $10 \AA$, valor considerado suficientemente grande para que a energia de interação seja desprezível. Para selecionar o parâmetro de impacto máximo $\left(b_{\max }\right)$, que conduz a reação, foi seguido o procedimento usual de calcular conjuntos de 100 trajetórias para cada energia translacional com parâmetro de impacto fixo. Em cada um destes conjuntos o parâmetro de impacto é acrescentado em $0.1 \AA$ de cada vez até atingir um valor onde em nenhuma das 100 trajetórias ocorre reação. Determinando assim o $b_{\max }$ com precisão de $0.1 \AA$. Após ser determinado o $b_{\max }$, são calculados conjuntos de 2000 trajetórias para cada combinação de energias translacional e rotacional. Esse número de trajetórias é suficiente para se obter uma seção de choque com um erro estatístico suficientemente baixo.

Para uma combinação de energia translacional $\left(E_{\text {tr }}\right)$ e rotacional (definida pelos números quânticos $j_{\mathrm{OH}}$ e $j_{\mathrm{SO}}$ ) a seção de choque específica é dada por:

$$
\sigma_{R}\left(E_{\mathrm{tr}} ; j_{\mathrm{OH}} ; j_{\mathrm{SO}}\right)=\pi b_{\max }^{2} P_{r}\left(E_{\mathrm{tr}} ; j_{\mathrm{OH}} ; j_{\mathrm{SO}}\right)
$$

com o erro estatístico:

$$
\Delta \sigma_{R}=\left(\frac{N_{T}-N_{r}}{N_{T} N_{r}}\right)^{1 / 2} \sigma_{R}
$$

$N_{r}$ é o número de trajetórias reativas do total de trajetórias calculadas, $N_{T}$, e $P_{r}=N_{r} / N_{T}$ é a probabilidade de reação. Como foi mostrado na seção (2.2), multiplicando a seção de choque $\sigma_{R}\left(E_{\mathrm{tr}} ; j_{\mathrm{OH}} ; j_{\mathrm{sO}}\right)$ pela energia translacional e integrando por uma distribuição de Boltzmann para a energia translacional, a constante de velocidade de reação específica obtém-se como:

$$
k\left(T ; j_{\mathrm{OH}} ; j_{\mathrm{SO}}\right)=g_{e}(T)\left(\frac{2}{k_{B} T}\right)^{3 / 2}\left(\frac{1}{\pi \mu}\right)^{1 / 2} \times \int_{0}^{\infty} E_{\mathrm{tr}} \sigma_{R}\left(E_{\mathrm{tr}} ; j_{\mathrm{OH}} ; j_{\mathrm{SO}}\right) \times \exp \left(-\frac{E_{\mathrm{tr}}}{k_{B} T}\right) d E_{\mathrm{tr}},
$$


onde $T$ é a temperatura, $\mu$ é a massa reduzida, $k_{B}$ é a constante de Boltzmann e $g_{e}(T)$ é o fator de degenerescência eletrônica, que tem a forma ${ }^{[58,119,120]}$ :

$$
g_{e}(T)=\frac{1}{3[1+\exp (-205 / T)]}
$$

Este fator é muito importante, pois na SEP não é levado em consideração o desdobramento dos estados eletrônicos (dos reagentes e do sistema $\mathrm{HSO}_{2}$ ) dado pela interação spin-órbita. Mas, nos resultados experimentais a reação ocorre numa ou várias dessas SEP desdobradas, dependendo da temperatura do sistema no experimento. Portanto, na ausência de uma descrição detalhada do acoplamento não adiabático dos estados eletrônicos, é necessário multiplicar e dividir a velocidade de reação obtida pelas multiplicidades da SEP e dos reagentes respectivamente, pois, somente uma parte das colisões calculadas descreve os resultados experimentais. Neste caso, o $\mathrm{OH}\left({ }^{2} \Pi\right)$ se desdobra em dois estados quase-degenerados, $\mathrm{OH}\left({ }^{2} \Pi_{1 / 2}\right)$ e $\mathrm{OH}\left({ }^{2} \Pi_{3 / 2}\right)$, enquanto o $\mathrm{SO}\left({ }^{3} \Sigma\right)$ é simplesmente o tripleto $\mathrm{SO}\left({ }^{3} \Sigma_{1}\right)$. Para baixas temperaturas só um dos dois estados do $\mathrm{OH}$ vai existir, sendo seis a multiplicidade de spin dos reagentes e sabendo que a SEP tem multiplicidade dois, fica $g_{e}=1 / 3$. Para altas temperaturas os dois estados do $\mathrm{OH}$ podem coexistir e a multiplicidade dos reagentes vai ser 12 , ficando $g_{e}=1 / 6$. A diferença energética entre os dois estados do $\mathrm{OH}$ é de $205 \mathrm{~K}^{[121]}$, daqui que a exponencial da equação (4.6) é uma medida da população do estado eletrônico mais alto do $\mathrm{OH}$.

Para monitorar a formação de estruturas moleculares formadas durante a colisão foram estudados conjuntos de 100 trajetórias para algumas combinações de números quânticos rotacionais e energias translacionais. Analisando as distâncias de ligação e os gráficos de energia potencial em função do tempo, foi encontrado que quando os átomos ficam próximos um dos outros (por distâncias menores de $3.5 \AA$ ) a energia potencial é menor que o limite de dissociação $\mathrm{H}+\mathrm{SO}_{2}$. Portanto, segundo o diagrama da SEP DMBE mostrado na figura 4.2, somente podem ser formados os compostos HOSO e $\mathrm{HSO}_{2}$, desde que o intervalo de energias translacionais estudado aqui vai aproximadamente desde 0.2 até $20 \mathrm{kcal} / \mathrm{mol}$. Mas, também foi verificado que a distância HS sempre fica maior que as distâncias $\mathrm{OH}$. Isso indica que a estrutura intermediária formada nas colisões é o composto HOSO. Dessa forma, foi implementado no código que a formação desta estrutura seja detectada quando as distâncias $\mathrm{HS}$ e $\mathrm{SO}_{a}\left(\mathrm{O}_{\mathrm{a}}\right.$ é o 
oxigênio do diátomo inicial $\mathrm{OH}$ ) sejam menores que 1.3 vezes seus correspondentes valores na geometria otimizada do HOSO. Essa definição conduz a tempos de vida da estrutura que concordam muito bem com os gráficos de energia potencial em função do tempo obtidos nos cálculos das trajetorias. A formação da estrutura $\mathrm{HSO}_{2}$ não foi observada.

Um problema bem conhecido no estudo de trajetórias quase-clássicas é a falta de descrição da energia de ponto zero (ZPE). Vários métodos têm sido propostos para resolver esse problema de forma aproximada ${ }^{[38,98]}$. Neste trabalho foi usado o método VEQMT ${ }^{[122]}$ (Vibrational Energy Quantum Mechanical Threshold). Esse é um método simples, onde somente são incluídas na análise estatística aquelas trajetórias onde a energia vibracional total dos produtos de reação excede a soma das correspondentes ZPEs.

\subsection{Resultados e discussão}

A tabela 4.3 mostra os resultados dos cálculos de trajetórias onde foram excitados rotacionalmente tanto um quanto ambos os reagentes. Nessa tabela, $N_{\text {com }}$ é o número de trajetórias que formam compostos intermediários, enquanto que, $N_{\text {rec }}$ é o número de trajetórias com recrossing, ou seja, são trajetórias que depois de formar composto retorna aos reagentes iniciais. Os resultados das trajetórias quase-clássicas calculadas (QCT na tabela 4.3) não consideram a correção do ZPE, enquanto no VEQMT sim é considerada. Para todas as energias translacionais e internas consideradas neste trabalho, se verifica que $N_{\text {com }}=N_{\text {rec }}+N_{r}$ (ver tabela 4.3). Portanto, todas as trajetórias reativas calculadas conduzem inicialmente a formação do composto HOSO. O tempo de vida médio deste composto depende tanto da energia translacional quanto da energia rotacional dos reagentes. Foram observados tempos de vida maiores para as combinações de estados quânticos rotacionais mais baixos e menores energias de colisão, onde é favorecida a reorganização da energia e, portanto, a formação de produtos de reação. Para combinações de maiores energias interna e translacional foram observados tempos de vida menores, produzindo menor reatividade. 
Tabela 4.3 Resumo dos cálculos de trajetórias para diferentes combinações de estados quânticos rotacionais dos reagentes.

\begin{tabular}{|c|c|c|c|c|c|c|c|c|c|c|}
\hline \multirow[t]{2}{*}{$\overline{j_{\mathrm{OH}}, j_{\mathrm{SO}}}$} & \multirow{2}{*}{$\begin{array}{c}E_{\mathrm{tr}} \\
\left(\mathrm{kcal} \mathrm{mol}^{-1}\right)\end{array}$} & \multirow{2}{*}{$\begin{array}{c}b_{\max } \\
\AA\end{array}$} & \multicolumn{4}{|c|}{$\mathrm{QCT}^{\mathrm{a}}$} & \multicolumn{4}{|c|}{ VEQMT $^{b}$} \\
\hline & & & $N_{T}$ & $N_{\text {com }}$ & $N_{\mathrm{rec}}$ & $N_{r}$ & $N_{T}$ & $N_{\text {com }}$ & $N_{\text {rec }}$ & $N_{r}$ \\
\hline \multirow{8}{*}{3,0} & 0.199 & 8.7 & 2000 & 1616 & 685 & 931 & 1290 & 1015 & 115 & 900 \\
\hline & 0.396 & 7.7 & 2000 & 1523 & 616 & 907 & 1341 & 980 & 115 & 865 \\
\hline & 0.596 & 7.1 & 1999 & 1460 & 603 & 857 & 1352 & 938 & 98 & 840 \\
\hline & 0.993 & 6.4 & 1996 & 1320 & 557 & 763 & 1405 & 863 & 128 & 735 \\
\hline & 1.987 & 5.4 & 1999 & 1300 & 705 & 595 & 1340 & 757 & 185 & 572 \\
\hline & 5.962 & 4.0 & 2000 & 1274 & 941 & 333 & 1282 & 700 & 383 & 317 \\
\hline & 9.936 & 3.7 & 2000 & 1213 & 1015 & 198 & 1372 & 727 & 532 & 195 \\
\hline & 19.872 & 2.8 & 2000 & 1256 & 1169 & 87 & 1493 & 956 & 869 & 87 \\
\hline \multirow[t]{8}{*}{6,0} & 0.199 & 8.7 & 2000 & 1398 & 655 & 743 & 1394 & 918 & 204 & 714 \\
\hline & 0.396 & 7.6 & 2000 & 1369 & 610 & 759 & 1424 & 924 & 179 & 745 \\
\hline & 0.596 & 7.1 & 2000 & 1288 & 623 & 665 & 1427 & 830 & 180 & 650 \\
\hline & 0.993 & 6.4 & 1999 & 1178 & 594 & 584 & 1477 & 752 & 188 & 564 \\
\hline & 1.987 & 5.4 & 2000 & 1112 & 630 & 482 & 1438 & 681 & 218 & 463 \\
\hline & 5.962 & 4.2 & 2000 & 1087 & 792 & 295 & 1427 & 636 & 351 & 285 \\
\hline & 9.936 & 3.7 & 2000 & 1123 & 919 & 204 & 1434 & 725 & 527 & 198 \\
\hline & 19.872 & 2.9 & 2000 & 1195 & 1078 & 117 & 1565 & 976 & 860 & 116 \\
\hline \multirow[t]{8}{*}{9,0} & 0.199 & 8.7 & 2000 & 1311 & 701 & 610 & 1483 & 929 & 335 & 594 \\
\hline & 0.396 & 7.6 & 2000 & 1261 & 692 & 569 & 1467 & 878 & 319 & 559 \\
\hline & 0.596 & 7.1 & 2000 & 1167 & 643 & 524 & 1526 & 817 & 299 & 518 \\
\hline & 0.993 & 6.3 & 2000 & 1139 & 643 & 496 & 1501 & 769 & 282 & 487 \\
\hline & 1.987 & 5.4 & 1999 & 988 & 580 & 408 & 1526 & 663 & 259 & 404 \\
\hline & 5.962 & 4.1 & 2000 & 1043 & 749 & 294 & 1491 & 719 & 429 & 290 \\
\hline & 9.936 & 3.7 & 2000 & 1064 & 877 & 187 & 1479 & 725 & 547 & 178 \\
\hline & 19.872 & 3.0 & 2000 & 1174 & 1067 & 107 & 1589 & 938 & 832 & 106 \\
\hline \multirow[t]{8}{*}{12,0} & 0.199 & 8.7 & 2000 & 1259 & 737 & 522 & 1585 & 968 & 447 & 521 \\
\hline & 0.396 & 7.8 & 2000 & 1121 & 687 & 434 & 1610 & 852 & 423 & 429 \\
\hline & 0.596 & 7.0 & 2000 & 1113 & 694 & 419 & 1574 & 818 & 406 & 412 \\
\hline & 0.993 & 6.3 & 2000 & 1002 & 620 & 382 & 1588 & 718 & 343 & 375 \\
\hline & 1.987 & 5.4 & 2000 & 893 & 581 & 312 & 1630 & 650 & 343 & 307 \\
\hline & 5.962 & 4.3 & 2000 & 861 & 619 & 242 & 1621 & 651 & 415 & 236 \\
\hline & 9.936 & 3.8 & 2000 & 956 & 779 & 177 & 1602 & 745 & 573 & 172 \\
\hline & 19.872 & 3.2 & 2000 & 1076 & 951 & 125 & 1630 & 901 & 777 & 124 \\
\hline \multirow[t]{7}{*}{1,10} & 0.199 & 8.8 & 2000 & 1664 & 596 & 1068 & 1391 & 1160 & 121 & 1039 \\
\hline & 0.396 & 7.6 & 2000 & 1650 & 622 & 1028 & 1336 & 1113 & 121 & 992 \\
\hline & 0.596 & 7.0 & 1999 & 1556 & 610 & 946 & 1318 & 1010 & 97 & 913 \\
\hline & 0.993 & 6.1 & 2000 & 1490 & 684 & 806 & 1257 & 912 & 128 & 784 \\
\hline & 1.987 & 5.3 & 1992 & 1319 & 685 & 634 & 1222 & 732 & 130 & 602 \\
\hline & 5.962 & 3.7 & 1998 & 1248 & 899 & 349 & 1265 & 705 & 367 & 338 \\
\hline & 9.936 & 3.4 & 2000 & 1217 & 988 & 229 & 1332 & 745 & 522 & 223 \\
\hline \multirow[t]{8}{*}{1,20} & 0.199 & 8.8 & 2000 & 1558 & 634 & 924 & 1272 & 1010 & 118 & 892 \\
\hline & 0.396 & 7.6 & 1999 & 1513 & 694 & 819 & 1237 & 929 & 145 & 784 \\
\hline & 0.596 & 7.0 & 1998 & 1524 & 625 & 899 & 1333 & 1001 & 133 & 868 \\
\hline & 0.993 & 6.1 & 2000 & 1479 & 701 & 778 & 1239 & 879 & 131 & 748 \\
\hline & 1.987 & 5.3 & 1992 & 1334 & 727 & 607 & 1248 & 762 & 182 & 580 \\
\hline & 5.962 & 3.7 & 2000 & 1253 & 909 & 344 & 1326 & 743 & 418 & 325 \\
\hline & 9.936 & 3.4 & 2000 & 1230 & 1036 & 194 & 1394 & 795 & 610 & 185 \\
\hline & 19.872 & 2.6 & 2000 & 1236 & 1139 & 97 & 1517 & 982 & 886 & 96 \\
\hline \multirow[t]{3}{*}{1,30} & 0.199 & 8.8 & 1998 & 1378 & 639 & 739 & 1261 & 878 & 166 & 712 \\
\hline & 0.396 & 7.6 & 1998 & 1390 & 633 & 757 & 1281 & 867 & 140 & 727 \\
\hline & 0.596 & 7.0 & 1998 & 1312 & 628 & 684 & 1270 & 812 & 158 & 654 \\
\hline
\end{tabular}




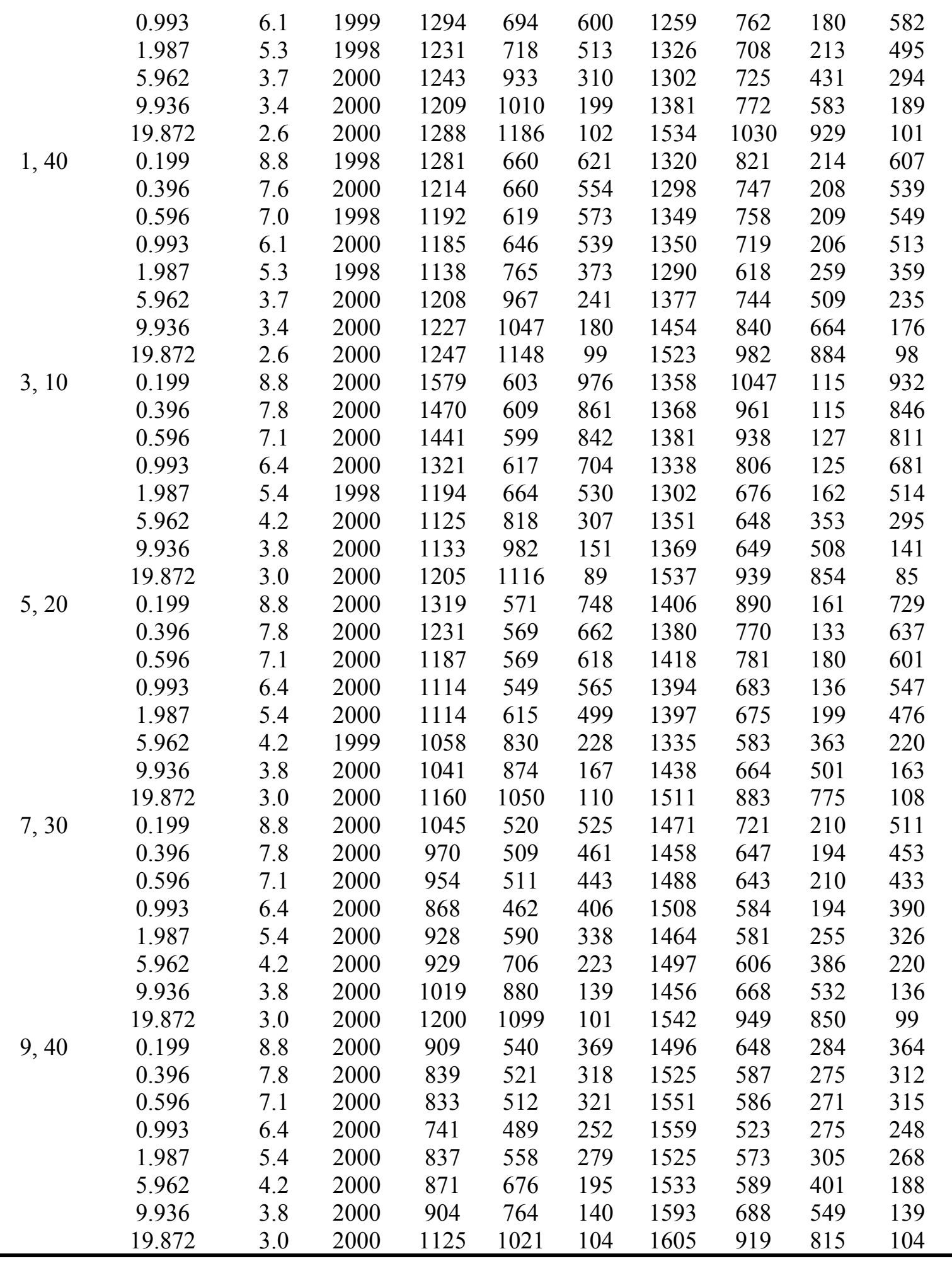

${ }^{\mathrm{a}}$ QCT (Quasi-classical trajectory) indica os resultados das trajetórias calculadas sem considerar a ZPE.

${ }^{\mathrm{b}}$ É considerada a ZPE.

A reação $\mathrm{OH}+\mathrm{SO}$ é controlada pelas forças multipolares de longo alcance. Os reagentes são capturados por um poço de energia potencial profundo, como indicado 
nas figuras 4.1 e 4.2, para formar o composto HOSO. Algumas trajetórias que formam o composto HOSO ficam oscilando ao redor desta configuração por muito tempo. Esta situação aumenta o custo computacional e produz algumas trajetórias não convergentes (menos do 2\%).

Os valores QCT mostrados na Tabela 4.3 são comparáveis com os obtidos no método VEQMT. Pode-se notar que a maior diferença entre os métodos aparece no número de trajetórias com recrossing que não satisfazem as condições impostas pelo método VEQMT. Esse efeito diminui quando aumenta a energia rotacional dos reagentes. Portanto, uma parte da energia rotacional é convertida em energia vibracional nas trajetórias não reativas. Como já foi feito em trabalhos anteriores ${ }^{[58]}$, os limites superiores e inferiores da reatividade são dados pelos resultados de QCT e VEQMT respectivamente.

Na figura 4.3 são mostrada as curvas de seção de choque em função da energia translacional (funções de excitação) para energias translacionais baixas. Nesse gráfico foram incluídas algumas combinações de estados rotacionais. As funções de excitação mostram um comportamento comum em reações de tipo captura ${ }^{[57,123]}$.

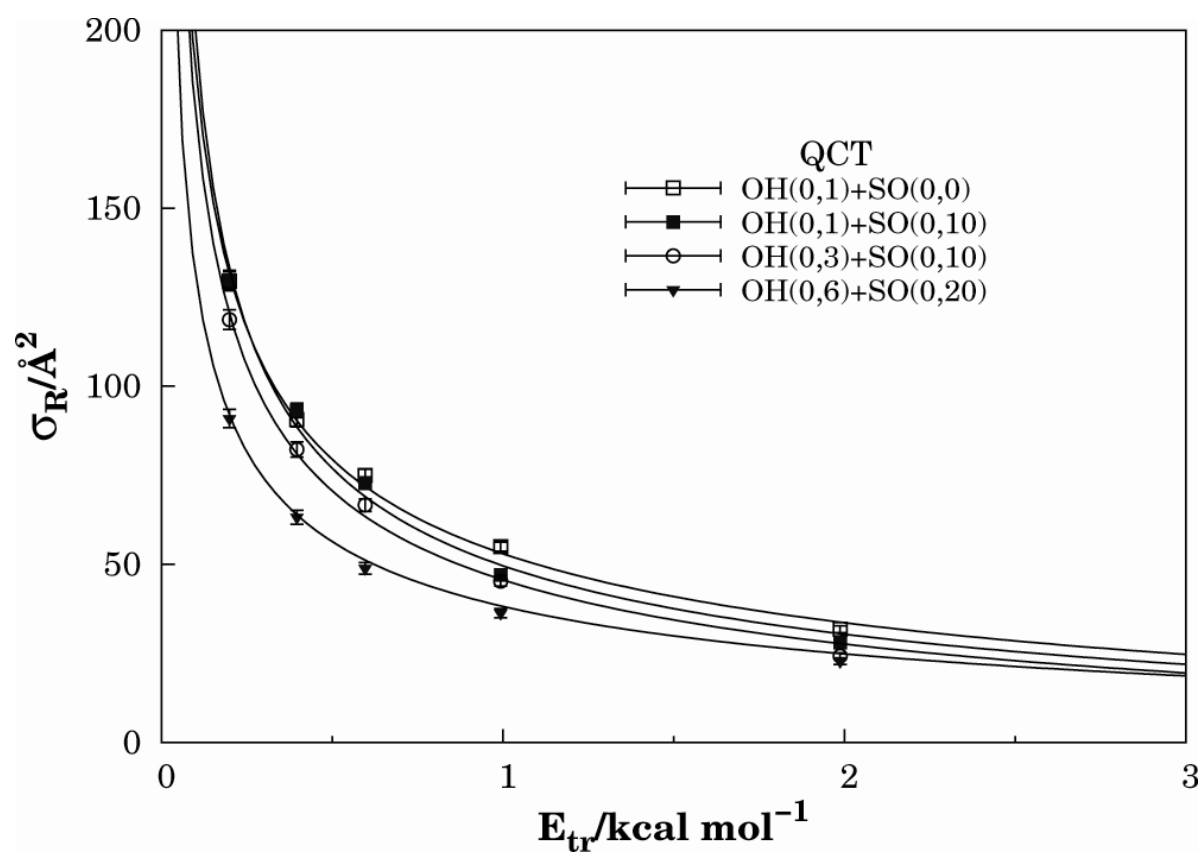

Figura 4.3. Funções de excitação para algumas combinações de estados rotacionais dos reagentes. As linhas representam o ajuste dos valores calculados às equações 4.7 e 4.8 .

Analisando a figura 4.3 parece evidente que a reatividade diminui na medida em que aumenta a energia rotacional. Isto é ilustrado também nos gráficos de seção de choque versus $j_{\mathrm{OH}}$ mostrados na figura 4.4. Nesses gráficos as seções de choque foram 
normalizadas aos valores correspondentes do nível rotacional fundamental $\left(j_{\mathrm{OH}}=1\right.$, $j_{\mathrm{SO}}=0$ ) e foram consideradas somente as energias translacionais mais baixas. As energias translacionais mais altas vão ser discutidas mais adiante nesta seção. Comparando os gráficos da figura 4.4 com os reportados para a reação $\mathrm{D}+\mathrm{H}_{2}{ }^{[124]}$, se apreciam comportamentos semelhantes, observando-se um decréscimo da reatividade relativa na medida em que $j$ aumenta. Porém, na reação $\mathrm{D}+\mathrm{H}_{2}$ é reportada uma mudança na monotonia das curvas, conduzindo à existência de mínimos ao redor de $j=5$, enquanto que, no presente estudo a reatividade sempre diminui quando $j$ aumenta. Comportamentos similares são observados quando a energia rotacional é depositada tanto no reagente $\mathrm{OH}$ quanto no $\mathrm{SO}$.
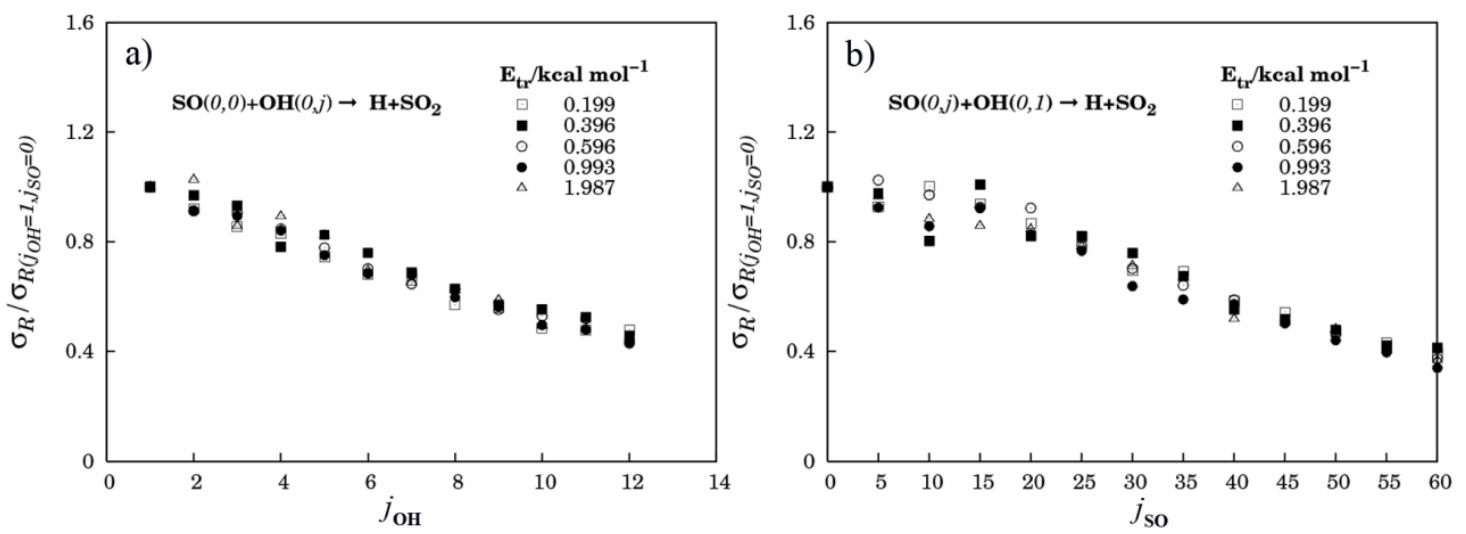

Figura 4.4. Seções de choque normalizadas em função dos estados quânticos rotacionais $j_{\mathrm{OH}}$ (a) e $j_{\text {So }}$ (b) para as cinco energias translacionais mais baixas estudas neste trabalho.

Os resultados mostrados são obtidos com somente um reagente rotacionalmente excitado e com energias translacionais menores que $2.0 \mathrm{kcal} \mathrm{mol}^{-1}$. Os gráficos da figura 4.5 mostram seções de choque QCT normalizadas em função da energia rotacional total dos reagentes. As linhas continuas indicam a tendência da seção de choque. De igual forma, não foram observadas diferenças quando a energia rotacional é depositada em um ou no outro reagente. Outra conclusão significativa é que o aumento da energia rotacional afeta principalmente a reatividade para energia translacional baixa, enquanto para energias translacionais altas o aumento da energia rotacional dos regentes tem pouco efeito na reatividade. Este comportamento é provavelmente provocado pelas mudanças nas interações de longo alcance devido à rotação, que é mais relevante para baixas energias translacionais, pois, neste caso, as moléculas têm mais tempo para se reorientar no transcurso da colisão. 

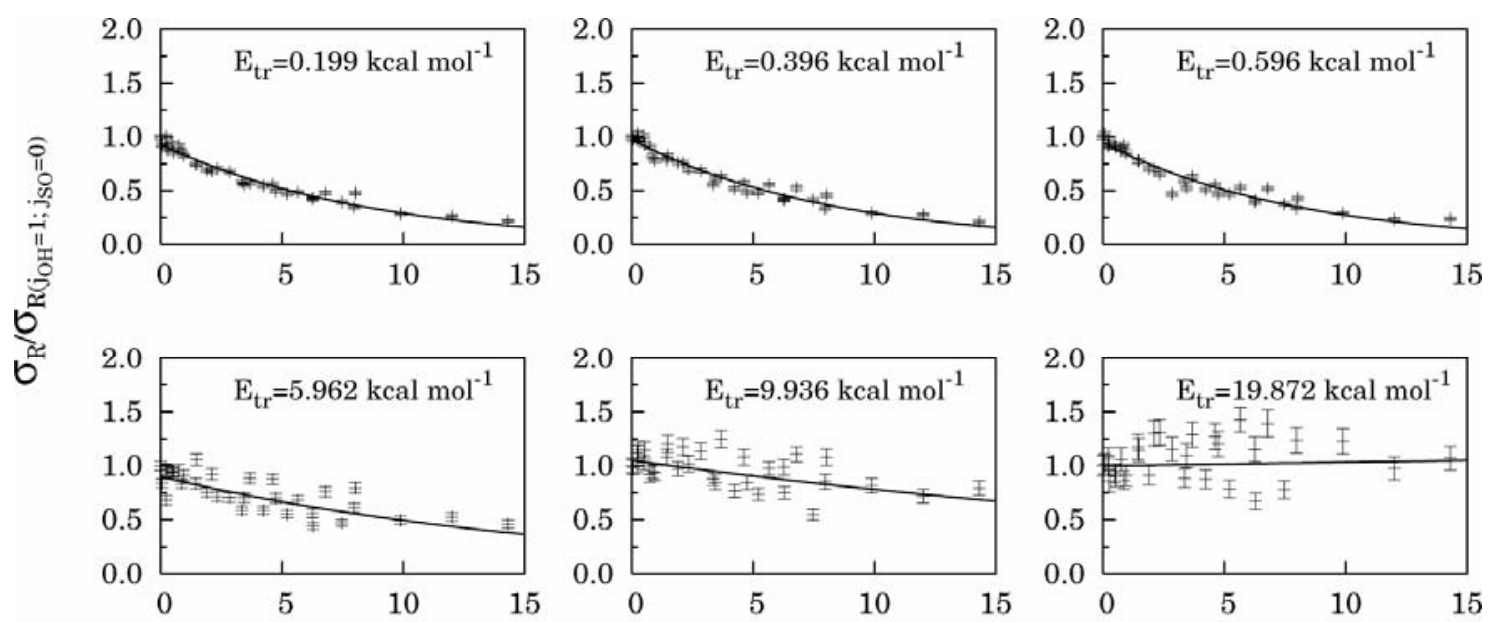

Energia de excitação rotacional total $/ \mathrm{kcal} \mathrm{mol}^{-1}$

Figura 4.5. Seções de choque normalizadas em função da energia rotacional total dos reagentes $\left(E_{\mathrm{rot}}^{\mathrm{OH}}+E_{\mathrm{rot}}^{\mathrm{SO}}\right)$. Os gráficos superiores representam as energias translacionais mais baixas, enquanto os inferiores as energias translacionais maiores. As linhas continuas indicam a tendência de cada conjunto de pontos.

Nas reações que ocorrem sem barreira de energia potencial, a seção de choque de

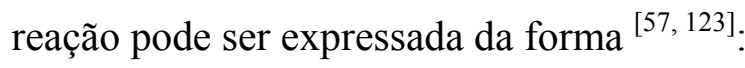

$$
\sigma\left(E_{\mathrm{tr}} ; E_{\mathrm{rot}}\right)=n \pi(n-2)^{(2-n) / n} \times\left(\frac{C_{n}}{2 E_{\mathrm{tr}}}\right)^{2 / n} \times F_{\mathrm{rec}}\left(E_{\mathrm{tr}} ; E_{\mathrm{rot}}\right) .
$$

O fator $F_{\text {rec }}\left(E_{\mathrm{tr}} ; E_{\mathrm{rot}}\right)$ considera os efeitos de recrossing, enquanto e o resto da expressão representa a seção de choque em reações de tipo captura, como foi discutido na seção (2.2). O fator de recrossing tem que ser levado em consideração, pois o modelo de reações de tipo captura, mostrado na seção (2.2), não permite voltar às condições iniciais uma vez que a distância entre os reagentes torna-se menor que a distância $R_{0}$, correspondente ao máximo do potencial efetivo. A dependência da seção de choque com a energia de rotação dos reagentes é representada pelo parâmetro $E_{\text {rot }}$, que denota a energia rotacional total dos reagentes. $O$ fator de recrossing $F_{\text {rec }}$, reportado em trabalhos anteriores ${ }^{[58]}$, depende da uma energia limiar $E_{\text {tr }}^{\text {th }}$ da forma:

$$
F_{\text {rec }}\left(E_{\text {tr }}\right)= \begin{cases}1 & E_{\text {tr }}<E_{\text {tr }}^{\text {th }} \\ \exp \left[-\alpha\left(E_{\mathrm{tr}}-E_{\text {tr }}^{\text {th }}\right)\right] & E_{\mathrm{tr}} \geq E_{\mathrm{tr}}^{\mathrm{th}}\end{cases}
$$


Nesse trabalho foi observado que quando os reagentes são excitados rotacionalmente a energia limiar é desprezível. Portanto, em vez do modelo usando anteriormente $^{[58]}$ foi proposto como fator de recrossing:

$$
F_{\text {rec }}\left(E_{\text {tr }}\right)=\exp \left[-\alpha\left(E_{\text {tr }}+E_{0}\right)\right]
$$

Esta expressão mostra que a probabilidade para que ocorra a reação, uma vez formado o composto intermediário, é inversamente proporcional a uma energia efetiva. Essa energia efetiva é dada pela soma da energia translacional inicial e uma energia que depende da energia interna dos reagentes $E_{\mathrm{tr}}+E_{0}$. Em outras palavras, a probabilidade de uma trajetória voltar aos reagentes depois da formação de composto $\left(P_{\text {rec }}=N_{\text {rec }} / N_{\text {com }}\right)$ aumenta quando a energia efetiva aumenta também. Ajustando a equação (4.9) a 1- $P_{\text {rec }}$ obtêm-se os parâmetros $\alpha$ e $E_{0}$. A Tabela 4.4 recolhe os coeficientes desses ajustes para QCT e VEQMT. Também são mostrados nessa tabela os valores de $n$ e $C_{n}$ da equação (4.7) que são ajustados aos valores de seções de choque obtidos da equação (4.3). Podese notar que para $n$ são obtidos valores entre os correspondentes a interações controladas por dipolo-dipolo $(n=3)$ e dipolo-quadrupolo $(n=4)$.

Tabela 4.4 Coeficientes obtidos dos ajustes das seções de choque e fatores de recrossing usando as equações (4.7) e (4.9) respectivamente. As unidades de medida dos coeficientes são aquelas para as quais $\sigma$ é expressada em $\AA^{2}$ enquanto $E_{\text {tr }}$ está em $\mathrm{kcal} \mathrm{mol}^{-1}$. Na segunda coluna é mostrada a energia rotacional total dos reagentes.

\begin{tabular}{cccccccccc}
\hline & & \multicolumn{4}{c}{ QCT } & \multicolumn{5}{c}{ VEQMT } \\
\cline { 3 - 9 }$j_{\mathrm{OH}, j \mathrm{SO}}$ & $E_{\mathrm{rot}}{ }^{\mathrm{a}}$ & $n$ & $C_{n}$ & $\alpha$ & $E_{0}{ }^{\mathrm{a}}$ & $n$ & $C_{n}$ & $\alpha$ & $E_{0}{ }^{\mathrm{a}}$ \\
\hline 1,0 & 0.000 & 3.9102 & 202.73 & 0.10 & 4.84 & 3.9763 & 213.79 & 0.09 & 0.86 \\
1,5 & 0.061 & 3.9499 & 205.40 & 0.08 & 6.43 & 3.9143 & 186.06 & 0.08 & 1.28 \\
2,0 & 0.212 & 3.9573 & 204.46 & 0.09 & 5.81 & 3.9530 & 190.38 & 0.08 & 1.49 \\
1,10 & 0.226 & 3.5667 & 113.85 & 0.10 & 4.81 & 3.9133 & 174.08 & 0.11 & 0.46 \\
2,5 & 0.273 & 3.9494 & 198.90 & 0.09 & 6.02 & 3.9481 & 167.88 & 0.10 & 0.92 \\
1,15 & 0.492 & 3.9499 & 186.76 & 0.12 & 3.77 & 3.9887 & 191.34 & 0.11 & 0.47 \\
3,0 & 0.529 & 3.9877 & 204.24 & 0.08 & 6.97 & 3.9482 & 160.81 & 0.11 & 0.69 \\
3,10 & 0.754 & 3.7349 & 129.55 & 0.13 & 3.77 & 3.7683 & 119.18 & 0.12 & 0.61 \\
1,20 & 0.861 & 3.9991 & 190.35 & 0.12 & 4.34 & 3.9593 & 170.83 & 0.12 & 0.69 \\
4,0 & 0.951 & 3.9539 & 177.39 & 0.09 & 6.44 & 3.9098 & 139.31 & 0.12 & 0.90 \\
4,15 & 1.443 & 3.9365 & 159.04 & 0.07 & 8.91 & 3.9653 & 129.18 & 0.12 & 0.98 \\
5,0 & 1.478 & 3.9986 & 173.01 & 0.07 & 8.64 & 3.9693 & 133.71 & 0.09 & 1.60 \\
1,30 & 1.905 & 3.9676 & 146.97 & 0.11 & 5.61 & 3.8916 & 121.49 & 0.11 & 1.49 \\
6,0 & 2.109 & 3.9756 & 150.38 & 0.08 & 8.00 & 3.9950 & 122.43 & 0.10 & 1.98 \\
5,20 & 2.339 & 3.7646 & 100.20 & 0.10 & 6.04 & 3.8013 & 86.30 & 0.11 & 1.51 \\
7,0 & 2.843 & 3.9240 & 127.74 & 0.11 & 5.70 & 3.8636 & 96.30 & 0.11 & 2.10 \\
1,40 & 3.355 & 3.8463 & 103.82 & 0.11 & 6.72 & 3.7207 & 76.37 & 0.11 & 2.62 \\
6,25 & 3.441 & 3.9734 & 110.59 & 0.08 & 8.81 & 3.9165 & 79.81 & 0.11 & 2.33
\end{tabular}




\begin{tabular}{cccccccccc}
8,0 & 3.679 & 3.9993 & 128.87 & 0.05 & 14.50 & 3.9613 & 107.73 & 0.08 & 4.28 \\
1,45 & 4.232 & 3.7524 & 82.32 & 0.12 & 6.41 & 3.6062 & 59.09 & 0.12 & 2.64 \\
9,0 & 4.617 & 3.9602 & 119.64 & 0.08 & 9.50 & 3.9998 & 106.24 & 0.09 & 4.36 \\
7,30 & 4.748 & 3.8629 & 73.41 & 0.10 & 7.18 & 3.7526 & 53.86 & 0.09 & 3.98 \\
1,50 & 5.209 & 3.9458 & 104.03 & 0.11 & 7.86 & 3.8870 & 83.63 & 0.10 & 4.69 \\
10,0 & 5.655 & 3.9583 & 118.65 & 0.06 & 14.45 & 3.9543 & 97.66 & 0.10 & 4.66 \\
8,35 & 6.259 & 3.7559 & 56.94 & 0.09 & 9.16 & 3.7140 & 44.47 & 0.10 & 4.66 \\
1,55 & 6.287 & 3.6460 & 72.65 & 0.09 & 11.34 & 3.7570 & 73.02 & 0.10 & 5.89 \\
11,0 & 6.792 & 3.9673 & 112.76 & 0.06 & 14.34 & 3.9665 & 98.15 & 0.06 & 9.41 \\
1,60 & 7.463 & 3.6808 & 75.79 & 0.10 & 10.92 & 3.5335 & 53.41 & 0.10 & 6.74 \\
9,40 & 7.972 & 3.8670 & 56.25 & 0.08 & 11.77 & 3.7751 & 41.84 & 0.10 & 5.79 \\
12,0 & 8.025 & 3.5592 & 60.47 & 0.07 & 12.90 & 3.4698 & 48.39 & 0.07 & 8.92 \\
10,45 & 9.887 & 3.9955 & 60.25 & 0.07 & 15.20 & 3.9359 & 48.64 & 0.07 & 10.84 \\
11,50 & 12.001 & 3.7634 & 41.41 & 0.07 & 16.08 & 3.6887 & 34.22 & 0.07 & 12.26 \\
\hline
\end{tabular}

${ }^{\mathrm{a}} \mathrm{Em} \mathrm{kcal} \mathrm{\textrm {mol } ^ { - 1 }}$

Depois de substituir a equação (4.7) em (4.5) e integrar, se obtêm as constantes de velocidade de reação específicas da forma:

$$
k(T)=k_{\text {cap }}(T) \frac{\exp \left(-\alpha E_{0}\right)}{\left(1+\frac{T}{T_{0}}\right)^{2(n-1) / n}}
$$

com

$$
k_{\text {cap }}(T)=2 n \pi g_{e}(T)(n-2)^{(2-n) / n} \times\left(\frac{2}{\pi \mu}\right)^{1 / 2}\left(k_{B} T\right)^{(n-4) / 2 n} \times\left(\frac{C_{n}}{2}\right)^{2 / n} \Gamma\left[\frac{2(n-1)}{n}\right],
$$

onde $T_{0}=1 /\left(\alpha k_{B}\right)$.

Para avaliar o papel da energia rotacional dos reagentes na reatividade de $\mathrm{OH}+\mathrm{SO}$, foi calculada uma constante de velocidade de reação média, usando a distribuição de população dos estados quânticos rotacionais em função da temperatura:

$$
P_{j}(T)=A(2 j+1) \exp \left(-\frac{B j(j+1)}{k_{B} T}\right)
$$

O fator $A$ é a constante de normalização que garante que

$$
\sum_{j=1}^{k} P_{j}(T)=1
$$

Portanto, a constante de velocidade de reação média é calculada como 


$$
\langle k\rangle=\sum_{j_{\mathrm{OH}=1}}^{12} \sum_{\mathrm{SO}=0}^{60} P_{j_{\mathrm{OH}}}(T) P_{j_{\mathrm{SO}}}(T) k\left(T ; j_{\mathrm{OH}} ; j_{\mathrm{SO}}\right) .
$$

Agora, em lugar de calcular todas as combinações de estados quânticos rotacionais na equação anterior, usamos a idéia de que os coeficientes de ajuste mostrados na tabela 4.4 dependem da energia rotacional total $E_{\text {rot }}$, sem importar como ela é distribuída nos reagentes. Portanto, cada conjunto de coeficientes foi ajustado a uma função linear dependente de $E_{\text {rot }}$, ficando:

$$
k\left(T ; j_{\mathrm{OH}} ; j_{\mathrm{SO}}\right)=k\left(T ; E_{\mathrm{rot}}\left(j_{\mathrm{OH}} j_{\mathrm{SO}}\right)\right) .
$$

Ou seja, para cada par de números quânticos $j_{\mathrm{OH}}$ e $j_{\mathrm{SO}}$ é conhecida a energia rotacional total $\left(E_{\mathrm{rot}}=E_{\mathrm{JOH}}+E_{\mathrm{JSO}}\right)$. Portanto, os valores $n$ e $C_{n}$ para cada par $j_{\mathrm{OH}}$ e $j_{\mathrm{SO}}$ podem ser obtidos dos ajustes feitos em função da $E_{\text {rot }}$.

Em seguida vamos comparar a constante de velocidade de reação média obtida com os resultados que aprecem na literatura. No estudo de colisões moleculares controladas por interações de longo alcance, Clary ${ }^{[125]}$ mostrou que na reação $\mathrm{OH}+\mathrm{O} \rightarrow \mathrm{H}+\mathrm{O}_{2}$, a dependência negativa da constante de velocidade de reação com a temperatura está relacionada com os efeitos rotacionais. Porém, Blitz et al. ${ }^{[106]}$ reportaram um decréscimo da constante de reação não linear com a aumento da temperatura, baseado na formação de dois compostos intermediários, $\mathrm{HOSO}$ e $\mathrm{HSO}_{2}$. Essa análise foi corroborada por estudos ab initio na Ref. ${ }^{[93]}$, concluindo que a isomerização $\mathrm{HOSO} \rightarrow \mathrm{HSO}_{2}$ forma parte do caminho de reação na formação dos produtos $\mathrm{H}+\mathrm{SO}_{2}$. Como foi reportado anteriormente ${ }^{[58]}$, e corroborado aqui, a formação do composto $\mathrm{HSO}_{2}$ não é observada nos nossos estudos de dinâmica quase-clássica. Em vez disso, é observada a dissociação direta desde o composto HOSO para os produtos de reação $\mathrm{H}+\mathrm{SO}_{2}$.

Na figura 4.6 é mostrada, na região sombreada, a constante de velocidade de reação média em função da temperatura. Como foi comentado anteriormente, o limite superior é dado pelos resultados VEQMT, enquanto o limite inferior é definido pelos QCT. Também são mostrados dados experimentais disponíveis na literatura. Levando em consideração a energia rotacional dos reagentes obtém-se uma dependência negativa com a temperatura mais pronunciada que a obtida anteriormente na Ref. ${ }^{[58]}$ para $j_{\mathrm{OH}}=1 \mathrm{e}$ $j_{\mathrm{SO}}=0$. Porém, não é observado o decaimento drástico reportado por Blitz et al. ${ }^{[106]}$ para 
temperaturas maiores que $500 \mathrm{~K}$. Nota-se que para temperaturas menores nossos resultados concordam razoavelmente bem com os dados experimentais.

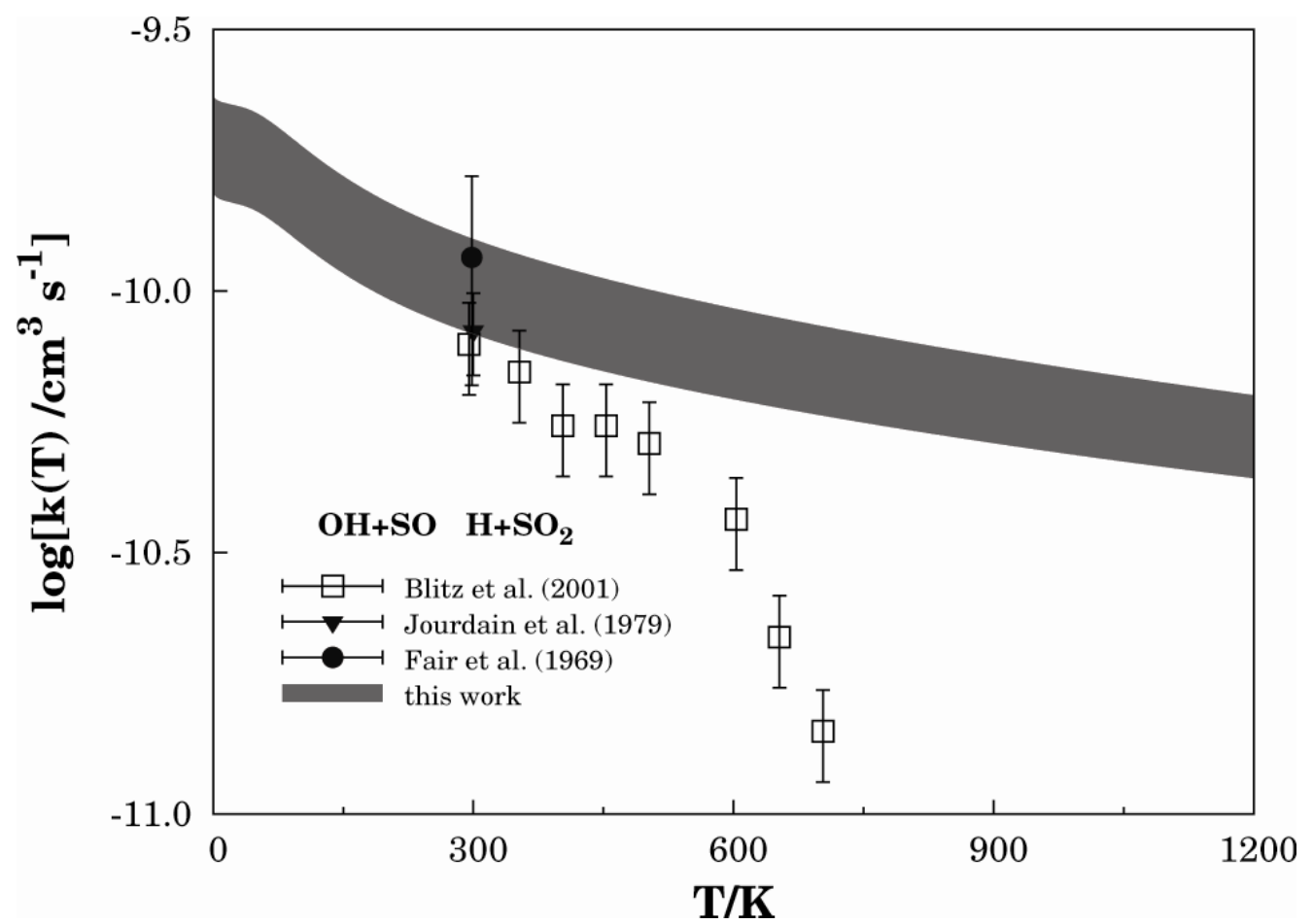

Figura 4.6. Constante de reação da colisão $\mathrm{OH}+\mathrm{O} \rightarrow \mathrm{H}+\mathrm{O}_{2}$. São apresentados dados experimentais com as correspondentes barras de erro. A região sombreada indica a constante de reação média obtida neste trabalho; os limites superiores e inferiores são definidos pelos resultados VEQMT e QCT respectivamente.

Para complementar os resultados, na tabela 4.5 são coletadas as constantes de reação experimentais e os resultados teóricos dos cálculos quase-clássicos obtidos nesse trabalho. Os resultados teóricos mostrados correspondem a constantes de velocidade de reação média e a constantes para $j_{\mathrm{OH}}=1, j_{\mathrm{SO}}=0$. Novamente os limites superior e inferior correspondem aos resultados VEQMT e QCT, respectivamente. Como foi mostrado na figura 4.5, para energias translacionais grandes não foram observados efeitos significativos na reatividade quando é aumentada a energia rotacional dos reagentes; enquanto para energias translacionais menores a reatividade diminui na medida em que aumenta a energia rotacional dos reagentes. 
Tabela 4.5 Constantes de reação (em $10^{-11} \mathrm{~cm}^{3} \mathrm{~s}^{-1}$ ) para a reação $\mathrm{OH}+\mathrm{SO}$. Os resultados experimentais são do trabalho de Blitz et al. ${ }^{[106]}$ exceto quando indicado.

\begin{tabular}{cccc}
\hline $\mathrm{T} / \mathrm{K}$ & $\mathrm{k}\left(\mathrm{j}_{\mathrm{OH}}=1, \mathrm{j}_{\mathrm{SO}}=0\right)$ & $\left\langle k\left(j_{\mathrm{OH}}, j_{\mathrm{SO}}\right)\right\rangle$ & Experimentais \\
\hline 295 & $8.9-13.7$ & $8.3-12.7$ & $7.91 \pm 1.58$ \\
298 & $8.9-13.6$ & $8.3-12.6$ & $11.6 \pm 5.0^{\mathrm{a}}$ \\
300 & $8.9-13.6$ & $8.3-12.6$ & $8.4 \pm 1.5^{\mathrm{b}}$ \\
353 & $8.4-12.9$ & $7.8-11.7$ & $7.00 \pm 1.40$ \\
403 & $8.1-12.3$ & $7.4-11.1$ & $5.53 \pm 1.10$ \\
453 & $7.8-11.9$ & $7.0-10.5$ & $5.53 \pm 1.10$ \\
503 & $7.5-11.5$ & $6.7-10.1$ & $5.11 \pm 1.02$ \\
553 & $7.3-11.2$ & $6.4-9.6$ & $4.45 \pm 0.89$ \\
603 & $7.1-10.9$ & $6.2-9.2$ & $3.66 \pm 0.73$ \\
653 & $7.0-10.6$ & $5.9-8.8$ & $2.18 \pm 0.43$ \\
703 & $6.8-10.3$ & $5.8-8.5$ & $1.44 \pm 0.30$ \\
\hline
\end{tabular}

${ }^{\mathrm{a}}$ Da Ref. ${ }^{[104]}$.

${ }^{\mathrm{b}}$ Da Ref. ${ }^{[105]}$.

\subsection{Conclusões}

O método de trajetórias quase-clássicas foi usado para estudar a colisão $\mathrm{OH}+\mathrm{SO}$. Nessa reação foi observado um comportamento de tipo captura junto a efeitos de recrossing. Foram estudados os efeitos das excitações rotacionais dos reagentes $\mathrm{OH}$ e $\mathrm{SO}$ na reatividade. Quando a energia rotacional é depositada tanto no reagente $\mathrm{OH}$ quanto no SO se obtém um decréscimo da reatividade, sendo observado que a seção de choque depende da energia rotacional total depositada nos reagentes. Os efeitos da rotação são mais relevantes para energias translacionais relativamente baixas. A constante de reação média reportada neste trabalho concorda razoavelmente bem com os dados experimentais obtidos para temperaturas menores que $500 \mathrm{~K}$, indicando que os efeitos rotacionais têm uma influência significativa na tendência negativa da constante de velocidade de reação com a temperatura. 


\section{Capítulo 5}

\section{Estudo da superfície de energia}

\section{potencial do sistema molecular $\mathrm{HSO}_{2}$}

\section{no nível CASPT2}

No capítulo anterior foi discutida a importância dos estudos relacionados ao sistema molecular $\mathrm{HSO}_{2}$. Como foi comentado também, na literatura foi publicada uma superfície de energia potencial global do estado eletrônico fundamental do sistema molecular $\mathrm{HSO}_{2}$, construída da teoria da expansão dupla de muitos corpos (DMBE) ${ }^{[98]}$. Esta superfície tem sido muito usada na determinação de constantes de velocidade de reações associadas a esta SEP. Mesmo obtendo-se bons resultados nos processos reativos estudados com essa SEP ${ }^{[58,59,110,111,126]}$, existe atualmente uma polêmica em relação a alguns caminhos de reação e estruturas estacionárias dessa superfície.

A superfície de energia potencial do estado fundamental do $\mathrm{HSO}_{2}$ pode ser dividida em duas regiões energéticas. A primeira, chamada aqui de região de baixas energias, inclui o mínimo global e os limites assintóticos $\mathrm{OH}+\mathrm{SO}$ e $\mathrm{H}+\mathrm{SO}_{2}$. A segunda, referida como região de maiores energias, envolve os isômeros vinculados aos limites de dissociação $\mathrm{HSO}+\mathrm{O}$ e $\mathrm{SH}+\mathrm{O}_{2}$. Nesses termos, podemos classificar em três grupos os trabalhos teóricos reportados sobre o sistema $\mathrm{HSO}_{2}$. O primeiro grupo é dedicado à 
descrição das estruturas estacionárias da região de baixas energias ${ }^{[87,92,95-97,127-136]}$. O segundo é composto por poucos trabalhos que estudam a região da SEP de maiores

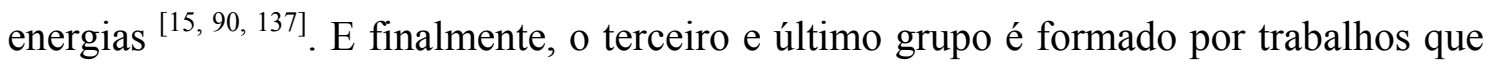
têm sido voltados à descrição de estruturas estacionárias das duas regiões, incluindo as

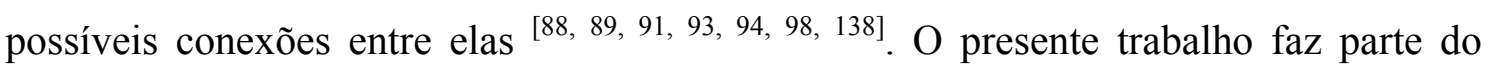
terceiro grupo. Uma análise mais detalhada destes trabalhos mencionados será feita posteriormente neste capítulo. Em relação ao sistema molecular $\mathrm{HSO}_{2}$, a opinião geral concorda que o mínimo global do estado fundamental é a estrutura chamada neste trabalho de $c i s-\mathrm{HOSO}_{\mathrm{s}}{ }^{[87-89,91-96,98,130-136]}$ e que em ordem crescente de energia, segue o mínimo local $\mathrm{HSO}_{2}$. A região de maiores energias, descrita na literatura, é formada pelos mínimos locais HSOO, cis-HOOS, trans-HOOS e os correspondentes estados de transição $[15,89,90,93,94,98,137,138]$. O que não está completamente claro ainda é o mecanismo de conexão entre as duas regiões mencionadas. Marshall et al. ${ }^{[94]}$, baseados em cálculos de mecânica quântica no nível MP2, indicaram que todas as tentativas de conectar os mínimos $\mathrm{HSOO}$ e $\mathrm{HSO}_{2}$ conduziam à dissociação $\mathrm{HSO}+\mathrm{O}$. Ballester e Varandas ${ }^{[98]}$, por outro lado, propuseram um estado de transição (TS) que conecta o mínimo de van der Waals HSO $\cdots$ O (da região de maiores energias) com o isômero $\mathrm{HSO}_{2}$ na SEP DMBE. Sendt et al. ${ }^{[15]}$ descartaram esta possibilidade, pois não conseguiram encontrar esse estado de transição usando cálculos de mecânica quântica no nível CASSCF.

Com tudo isso, o objetivo do presente trabalho é fazer uma descrição teórica na fase gasosa da topologia geral da SEP do $\mathrm{HSO}_{2}$ no estado fundamental com um alto nível de cálculo. Baseados nos resultados obtidos neste trabalho podem ser feitas correções na SEP DMBE ${ }^{[98]}$. Posteriormente, sabendo que a presença de água em fase condensada na troposfera pode influir notavelmente na reatividade desse sistema molecular, pode-se estudar o efeito do meio na altura das barreiras e na estabilidade das estruturas estacionárias.

\subsection{Métodos computacionais}

Os cálculos de mecânica quântica reportados neste trabalho são obtidos da teoria perturbativa de segunda ordem (CASPT2) ${ }^{[26,30]}$ usando a função de onda de referência CASSCF $^{[24,25]}$. Todos os cálculos de otimização de geometrias, limites de dissociação, 
energias de ponto zero e frequências harmônicas de vibração foram realizados neste nível de cálculo usando o pacote de programas MOLPRO 2008.1 ${ }^{[139]}$. No espaço ativo dos cálculos CASSCF foram considerados todos os orbitais moleculares formados pelos orbitais de valência de cada átomo (FVCAS, Full Valence Complete Active Space). Em relação ao conjunto de funções-base usado, foram levadas em consideração algumas dificuldades que apresentam as bases de Dunning, aug-cc-pVXZ, para descrever sistemas moleculares que contem átomos de enxofre ${ }^{[140-143]}$. Portanto, para o átomo de enxofre foram usadas as bases de Dunning acrescentadas de uma função $d$, referida como aug-cc-pV(X+d)Z ${ }^{[144]}$. Para os átomos de oxigênio e hidrogênio foram usadas as bases aug-cc-pVXZ. As otimizações de geometria foram realizadas com $\mathrm{X}=\mathrm{T}$ nos conjuntos de bases mencionados. A fim de evitar o problema de erro por superposição de bases, além de obter resultados mais precisos, as energias das estruturas estacionárias foram extrapoladas a um conjunto de funções-base infinito. Na literatura aparecem varias formas de extrapolação da energia ${ }^{[136-138,145]}$, mas, neste trabalho isso foi feito segundo a aproximação usada por Dixon et al. ${ }^{[138]}$, onde é assumido que a convergência da energia é dominada pelo comportamento da energia de correlação. Assim, para cada estrutura otimizada foi calculada a energia usando três conjuntos de bases diferentes, aug-cc-pVXZ com X=D, T, Q, a fim de ajustar a seguinte função de extrapolação ${ }^{[146]}$ às energias obtidas,

$$
E_{X}^{\mathrm{tot}}=E_{\infty}+B \exp [-(X-1)]+C \exp \left[-(X-1)^{2}\right]
$$

$E_{\infty}, B, C$ são os parâmetros de ajuste e $\mathrm{X}$ é igual a 2, 3 e 4 para $\mathrm{DZ}, \mathrm{TZ}$ e QZ respectivamente. $\mathrm{O}$ valor estimado da energia no limite do conjunto de bases infinitas é $E_{\infty}$

A conexão entre duas estruturas de mínimos através de um estado de transição é determinada da análise do modo normal vibracional associado à frequência imaginária do estado de transição e de resultados de caminhos de mínima energia que começam da estrutura do estado de transição até as estruturas de mínimo. Nos cálculos de otimização de geometrias, em alguns casos foram usadas geometrias de partida obtidas de outros trabalhos reportados ${ }^{[15,98]}$ ou começando de estruturas próximas dos possíveis mínimos. Os limites de dissociação foram calculados realizando otimizações parciais de geometria mantendo fixa a coordenada característica da dissociação de interesse. 


\subsection{Resultados e discussão}

Após uma análise detalhada das estruturas estacionárias (mínimos e estados de transição) obtidas dos cálculos realizados, foi elaborado o diagrama energético da superfície de energia potencial do sistema molecular $\mathrm{HSO}_{2}$. Nas figuras 5.1 e 5.2 são apresentados os diagramas energéticos dos principais caminhos de reação deste sistema molecular. A figura 5.1 envolve as reações $\mathrm{SH}+\mathrm{O}_{2} \rightarrow \mathrm{HSO}+\mathrm{O}, \mathrm{SH}+\mathrm{O}_{2} \rightarrow \mathrm{OH}+\mathrm{SO}$ e $\mathrm{SH}+\mathrm{O}_{2} \rightarrow \mathrm{SO}_{2}+\mathrm{H}$, enquanto na figura 5.2 aparece a reação $\mathrm{SH}+\mathrm{O}_{2} \rightarrow \mathrm{OH}+\mathrm{SO}$. Nestes diagramas são mostradas as conexões entre as estruturas estacionárias e os correspondentes estados de transição. As energias mostradas nos gráficos (a) não incluem a correção da energia de ponto zero, enquanto nos gráficos (b) a energia de cada estrutura leva em consideração o correspondente valor do ZPE.

Nas figuras 5.3 e 5.4 aparecem representadas esquematicamente as estruturas estacionárias indicadas nos diagramas energéticos. A figura 5.3 corresponde às estruturas de mínimo, enquanto na figura 5.4 são mostrados os estados de transição. As setas, nessa figura, indicam o modo normal vibracional correspondente à frequência imaginária.

A tabela 5.1 resume os valores energéticos das estruturas estacionárias e limites de dissociação da região de maiores energias da SEP. Na terceira coluna são indicados os valores das energias de ponto zero. A quarta coluna mostra os valores de energia de cada estrutura em relação à estrutura anterior, enquanto na quinta coluna os valores de energias são referidos ao limite de dissociação $\mathrm{SH}+\mathrm{O}_{2}$, em ambos os casos é incluída a correção do ZPE. De forma semelhante, a tabela 5.2 mostra os resultados obtidos para as estruturas estacionárias da região de baixas energias na SEP. Finalmente, a tabela 5.3 contém todas as frequências calculadas para cada uma das estruturas.

A seguir, serão discutidas as características dos principais caminhos de reação e propriedades gerais das estruturas estacionárias. 

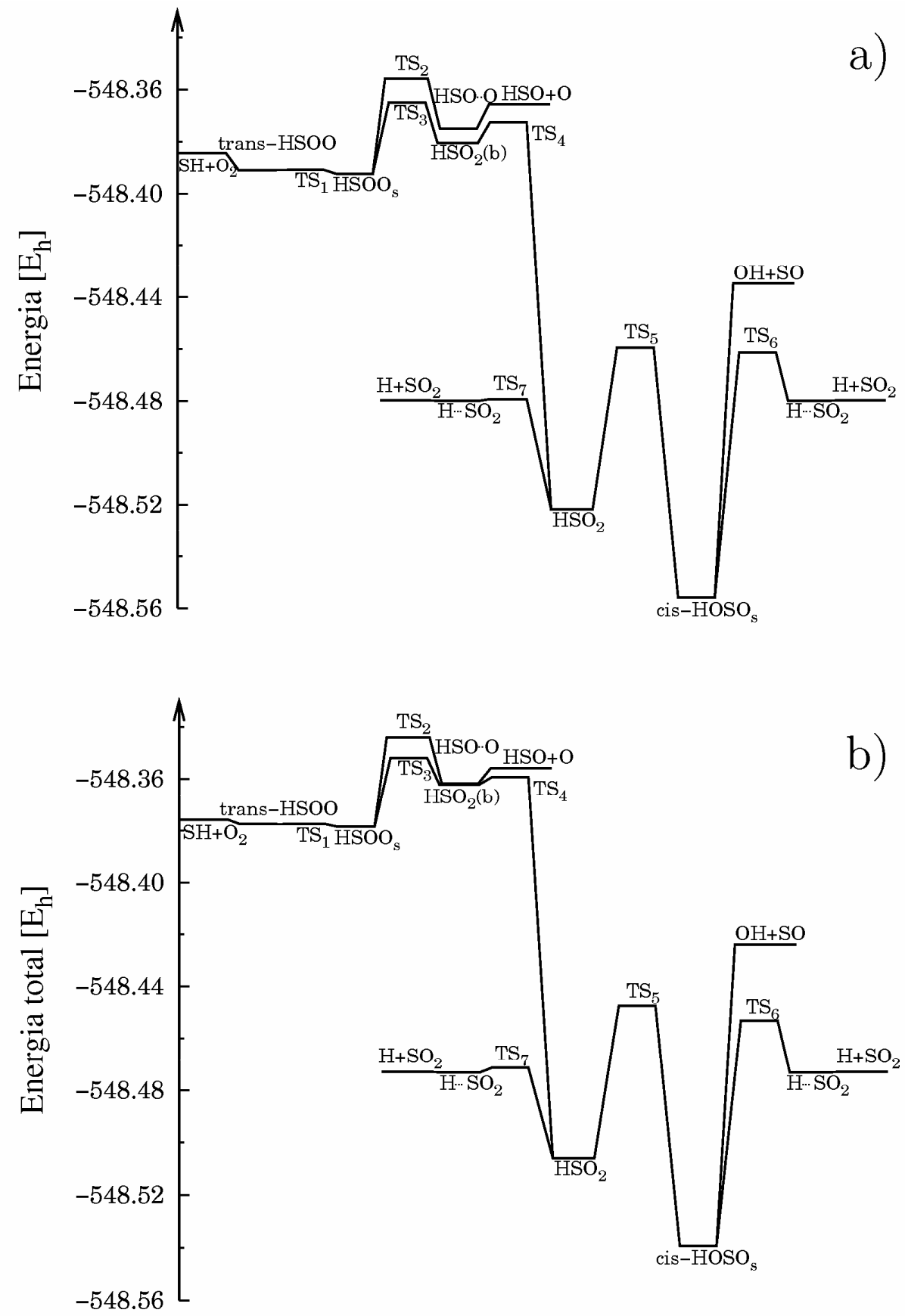

Figura 5.1. Diagrama energético obtido dos cálculos realizados, onde são mostrados os caminhos de reação $\mathrm{SH}+\mathrm{O}_{2} \rightarrow \mathrm{HSO}+\mathrm{O}, \mathrm{SH}+\mathrm{O}_{2} \rightarrow \mathrm{OH}+\mathrm{SO}$ e $\mathrm{SH}+\mathrm{O}_{2} \rightarrow \mathrm{SO}_{2}+\mathrm{H}$. Painel (a), sem levar em consideração o ZPE e painel (b), incluindo ZPE na energia total. 

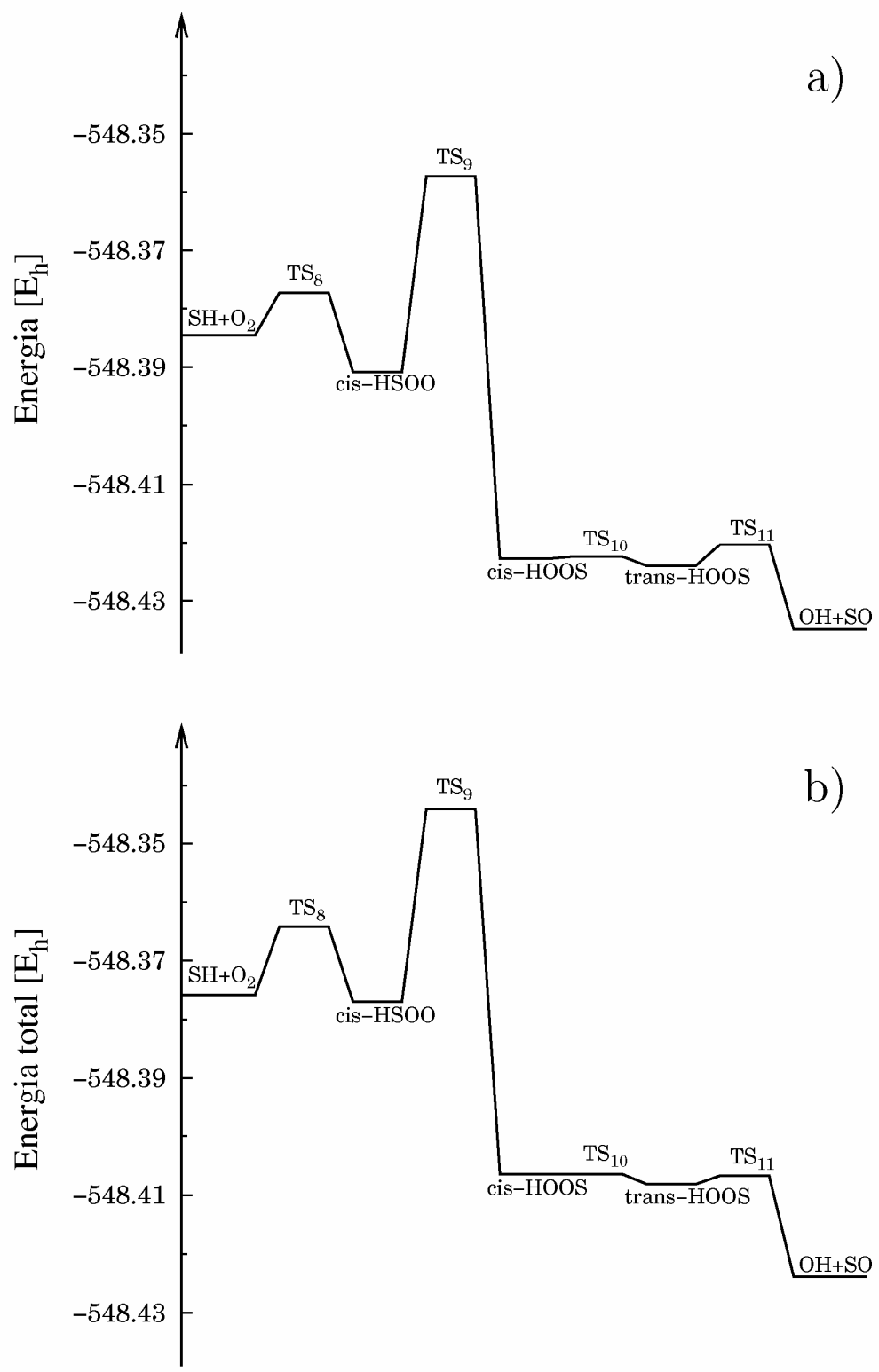

Figura 5.2. Diagrama energético obtido dos cálculos realizados onde é mostrado o caminho de reação $\mathrm{SH}+\mathrm{O}_{2} \rightarrow \mathrm{OH}+\mathrm{SO}$. Painel (a), sem levar em consideração o ZPE e painel (b), incluindo ZPE na energia total.

\subsubsection{Caminho de reação $\mathrm{SH}+\mathrm{O}_{2} \rightarrow \mathrm{HSO}+\mathrm{O}$}

$\mathrm{O}$ caminho de reação correspondente a $\mathrm{SH}+\mathrm{O}_{2} \rightarrow \mathrm{HSO}+\mathrm{O}$ forma parte da região de

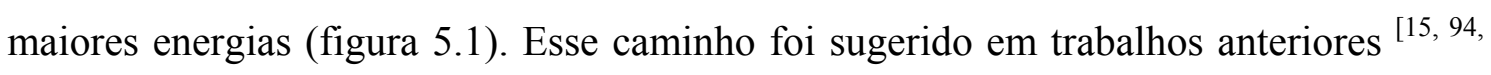
98, 137] , porém, existem algumas diferenças em comparação com os resultados obtidos no presente trabalho. Na Ref. ${ }^{[94]}$ não foi encontrado o estado de transição que liga o 
mínimo local $\mathrm{HSOO}_{\mathrm{s}}$ (s, do inglês skewed) ao limite de dissociação $\mathrm{HSO}+\mathrm{O}$. Por outro lado, Sendt et al. ${ }^{[15]}$ e Resende e Ornellas ${ }^{[137]}$ reportaram um mesmo estado de transição $\left(\mathrm{TS}_{1}\right)$ para ligar esse mínimo $\left(\mathrm{HSOO}_{\mathrm{s}}\right)$ ao outro limite de dissociação $\mathrm{SH}+\mathrm{O}_{2}$. Os resultados obtidos neste trabalho descrevem uma situação um pouco diferente. Segundo nossos cálculos, o estado de transição $\mathrm{TS}_{1}$ é incluído na reação $\mathrm{SH}+\mathrm{O}_{2} \rightarrow \mathrm{HSO}+\mathrm{O}$ formando uma pequena barreira no sentido da rotação do ângulo de torção H-S-O-O. Esta rotação transforma o mínimo planar trans-HSOO no isômero $\mathrm{HSOO}_{\mathrm{s}}$ através de uma pequena barreira de energia potencial. Um estado de transição semelhante aparece reportado na Ref. ${ }^{[98]}$, com um comprimento da ligação SO um pouco maior do que a nossa (aproximadamente $0.5 \AA$ ) e uma altura da barreira também maior. Os outros estados de transição que conduzem ao mínimo local $\mathrm{HSOO}_{\mathrm{s}}$ serão discutidos mais adiante.

As propriedades geométricas e frequências harmônicas calculadas na estrutura estacionária $\mathrm{HSOO}_{\mathrm{s}}$ (figura 5.3) concordam muito bem com cálculos recentes de Dixon et al. ${ }^{[138]}$ no nível $\operatorname{CCSD}(\mathrm{T}) / \mathrm{aug}-\mathrm{cc}-\mathrm{pV}(\mathrm{T}+\mathrm{D}) \mathrm{Z}$. A energia desse mínimo é 1.73 $\mathrm{kcal} / \mathrm{mol}$ abaixo do limite de dissociação $\mathrm{SH}+\mathrm{O}_{2}$ (tabela 5.1), um pouco acima do reportado por Dixon ${ }^{[138]}$ de $7.1 \mathrm{kcal} / \mathrm{mol}$. Porém, o valor concorda razoavelmente bem com o resultado de Zhou de $0.31 \mathrm{kcal} / \mathrm{mol}$ no nível MRCI/aug-cc-pV(Q+d)Z ${ }^{[15]}$. As características geométricas desse mínimo também se assemelham muito bem com as geometrias reportadas nas Refs. ${ }^{[15,89,93,98,137]}$, com diferenças menores do que $7 \%$. Toda a análise dos resultados energéticos no texto inclui a correção do ZPE. No trabalho de Dixon ${ }^{[138]}$ é reportada uma estrutura de mínimo chamada HSOO $_{t}$, que concorda com nossa estrutura trans-HSOO em um 97\%, em relação às propriedades geométricas e de frequências, com exceção de uma frequência imaginária muito baixa que aparece em nosso cálculo (tabela 5.3).

Ao longo do caminho de reação de $\mathrm{SH}+\mathrm{O}_{2} \rightarrow \mathrm{HSO}+\mathrm{O}$, o mínimo local $\mathrm{HSOO}_{\mathrm{s}}$ é seguido do estado de transição $\mathrm{TS}_{2}$ (figura 5.1), que o liga à estrutura $\mathrm{HSO} \cdot \mathrm{O}$. Nessa notação, os pontos indicam um afastamento dos átomos de oxigênio da ordem de $2.55 \AA$ e não é considerada uma estrutura de van der Waals devido à grande diferença energética em relação ao limite de dissociação $\mathrm{HSO}+\mathrm{O}$. A estrutura $\mathrm{TS}_{2}$ fica acima das estruturas $\mathrm{HSOO}_{\mathrm{s}}$ e $\mathrm{HSO} \cdot \mathrm{O}$ por 21.77 e $11.16 \mathrm{kcal} / \mathrm{mol}$ respectivamente, mostrando uma diferença de $20.04 \mathrm{kcal} / \mathrm{mol}$ em relação ao limite $\mathrm{SH}+\mathrm{O}_{2}$ e $7.49 \mathrm{kcal} / \mathrm{mol} \mathrm{em}$ comparação a $\mathrm{HSO}+\mathrm{O}$. Esses resultados confirmam o consenso geral que a formação dos produtos $\mathrm{HSO}+\mathrm{O}$ é um processo endoenergético. 


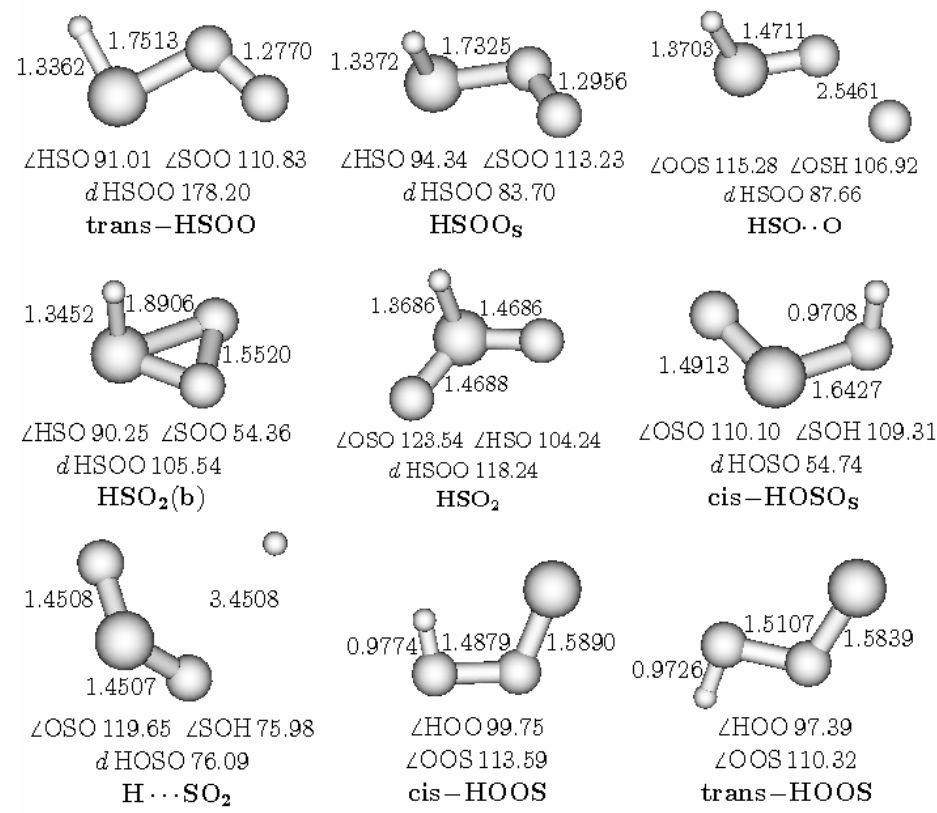

Figura 5.3. Representação esquemática das estruturas de mínimo e características geométricas obtidas nas otimizações de geometria.

O estado de transição $\mathrm{TS}_{2}$ foi reportado anteriormente ${ }^{[15,98]}$. A pesar das características geométricas (ver figura 5.4) desse estado de transição serem muito similares às reportadas na Ref. ${ }^{[15]}$, os valores de frequências mostrados na tabela 5.3 são um pouco diferentes, principalmente o correspondente à frequência imaginária. A separação energética em relação ao mínimo $\mathrm{HSOO}_{\mathrm{s}}$, antes mencionada de 21.77 $\mathrm{kcal} / \mathrm{mol}$, concorda muito bem com os valores 19.34 e $21.53 \mathrm{kcal} / \mathrm{mol}$ obtidos de cálculos CASSCF e MRCI respectivamente na Ref. ${ }^{[15]}$. Porém, no sentido inverso, nossa barreira $\mathrm{HSO} \cdot \mathrm{O} \rightarrow \mathrm{TS}_{2}$, de $11.16 \mathrm{kcal} / \mathrm{mol}$, é bem maior que os valores reportados na Ref. ${ }^{[15]}$ de 3.68 e $0.11 \mathrm{kcal} / \mathrm{mol}$ nos níveis de cálculo CASSCF e MRCI respectivamente.

As características geométricas do estado de transição $\mathrm{TS}_{2}$ concordam muito bem com a geometria obtida na Ref. ${ }^{[98]}$. Nesta referência, a altura da barreira em relação ao mínimo $\mathrm{HSO} \cdot \mathrm{O}$ é de $7.2 \mathrm{kcal} / \mathrm{mol}$, não muito diferente do nosso resultado (11.16 $\mathrm{kcal} / \mathrm{mol}$ ). No sentido contrário da reação, a altura desse estado de transição em comparação ao mínimo $\mathrm{HSOO}_{\mathrm{s}}$ e à dissociação $\mathrm{SH}+\mathrm{O}_{2}$, é respectivamente 8.40 e 9.29 $\mathrm{kcal} / \mathrm{mol}$, apresentando alguma diferenças em relação a nossos resultados. 


\subsubsection{Caminhos de reação $\mathrm{SH}+\mathrm{O}_{2} \rightarrow \mathrm{OH}+\mathrm{SO}$ (região de baixas energias), $\mathrm{SH}+\mathrm{O}_{2} \rightarrow \mathrm{H}+\mathrm{SO}_{2}$ e $\mathrm{OH}+\mathrm{SO} \rightarrow \mathrm{H}+\mathrm{SO}_{2}$}

$\mathrm{Na}$ figura 5.1 é mostrado o caminho de reação de $\mathrm{SH}+\mathrm{O}_{2} \rightarrow \mathrm{OH}+\mathrm{SO}$ que passa pelo mínimo global cis-HOSO${ }_{s}$. Nesse caminho os mínimos $\mathrm{HSOO}_{\mathrm{s}}$ e $\mathrm{HSO}_{2}(\mathrm{~b})$ são conectados pelo estado de transição $\mathrm{TS}_{3}$, seguidos do $\mathrm{TS}_{4}$ que os liga com o mínimo $\mathrm{HSO}_{2}$. $\mathrm{O}$ modo normal vibracional associado à frequência imaginária do estado de transição $\mathrm{TS}_{3}$ é representado na figura 5.4 pelas direções das setas. Este modo normal permite a ligação do átomo de oxigênio mais afastado ao átomo de enxofre para formar a estrutura $\mathrm{HSO}_{2}\left(\right.$ b). Posteriormente, o $\mathrm{TS}_{4}$ conduz à formação de $\mathrm{HSO}_{2}$ acrescentando a distância entre os oxigênios e ficando cada um deles ligados ao enxofre. Os detalhes das mudanças de estruturas são mostrados nas figuras 5.3 e 5.4. O estado de transição $\mathrm{TS}_{3}$ tem uma diferença energética de $16.76 \mathrm{kcal} / \mathrm{mol}$ em relação à estrutura $\mathrm{HSOO}_{\mathrm{s}} \mathrm{e}$ $6.40 \mathrm{kcal} / \mathrm{mol}$ comparado com $\mathrm{HSO}_{2}$ (b), como é mostrado na tabela 5.2, enquanto o $\mathrm{TS}_{4}$ apresenta 1.74 e $92.02 \mathrm{kcal} / \mathrm{mol}$ relativo aos mínimos $\mathrm{HSO}_{2}(\mathrm{~b})$ e $\mathrm{HSO}_{2}$ respectivamente.

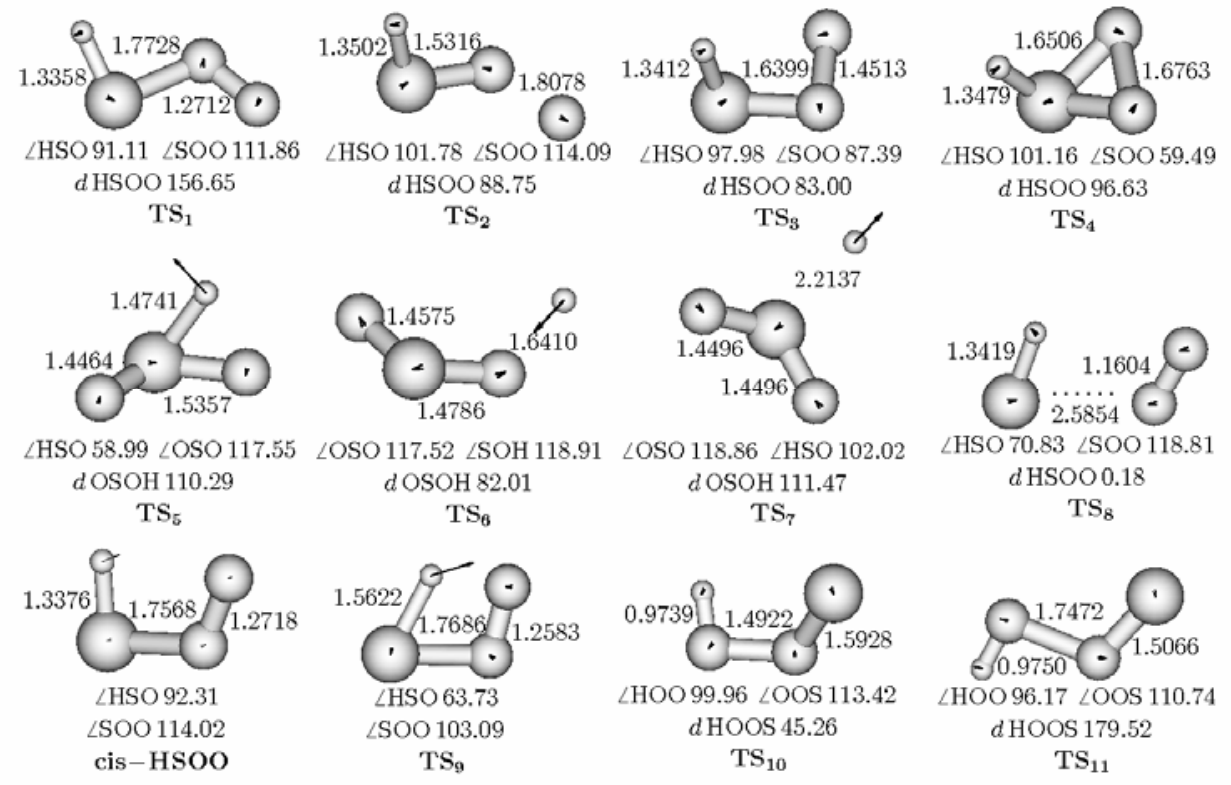

Figura 5.4. Representação esquemática dos estados de transição e característica geométricas obtidas das otimizações de geometria. As setas indicam o modo normal vibracional correspondente à frequência imaginária.

Na superfície de energia potencial DMBE ${ }^{[98]}$ foi indicado um estado de transição que conecta as estruturas $\mathrm{HSO} \cdot \mathrm{O}$ e $\mathrm{HSO}_{2}$, mas a existência desse estado de transição 
não foi verificada na Ref. ${ }^{[15]}$ no nível de cálculo CASSCF. De forma semelhante, nas Refs. ${ }^{[94,137]}$ não foi encontrado um estado de transição que ligue os reagentes $\mathrm{SH}+\mathrm{O}_{2}$ à formação da estrutura $\mathrm{HSO}_{2}$. Portanto, um estado de transição com as características de $\mathrm{TS}_{4}$ não foi reportado anteriormente.

Tabela 5.1: Propriedades energéticas dos pontos estacionários da região de maiores energias no diagrama energético.

\begin{tabular}{|c|c|c|c|c|}
\hline $\begin{array}{c}\text { Pontos } \\
\text { estacionários }\end{array}$ & Energia total (au). & ZPE (au) & $\begin{array}{l}\text { Diferença com a } \\
\text { estrutura anterior } \\
\quad(\mathrm{kcal} / \mathrm{mol}) .\end{array}$ & $\begin{array}{c}\text { Diferença com o } \\
\text { limite } \mathrm{SH}+\mathrm{O}_{2} \\
(\mathrm{kcal} / \mathrm{mol}) .\end{array}$ \\
\hline \multicolumn{5}{|c|}{ Caminho de reação $\mathrm{HS}+\mathrm{O}_{2} \rightarrow \mathrm{OH}+\mathrm{SO}$} \\
\hline $\mathrm{HS}+\mathrm{O}_{2}$ & -548.38457423 & 0.00872692 & 0.0 & 0.0 \\
\hline $\mathrm{TS}_{8}$ & -548.37729620 & 0.01313236 & +7.33 & +7.33 \\
\hline cis-HSOO & -548.39078414 & 0.01382676 & -8.03 & -0.70 \\
\hline $\mathrm{TS}_{9}$ & -548.35732700 & 0.01337374 & +20.71 & +20.01 \\
\hline cis-HOOS & -548.42283775 & 0.01641262 & -39.20 & -19.19 \\
\hline TS10 & -548.42242142 & 0.01599226 & -0.003 & -19.19 \\
\hline trans-HOOS & -548.42397423 & 0.01583925 & -1.07 & -20.26 \\
\hline $\mathrm{TS}_{11}$ & -548.42032895 & 0.01361613 & +0.89 & -19.37 \\
\hline $\mathrm{OH}+{ }^{3} \mathrm{SO}$ & -548.43485120 & 0.0109856 & -10.76 & -30.13 \\
\hline \multicolumn{5}{|c|}{ Caminho de reação $\mathrm{HS}+\mathrm{O}_{2} \rightarrow \mathrm{O}+\mathrm{HSO}$} \\
\hline $\mathrm{HSOO}_{\mathrm{s}}$ & -548.39242944 & 0.01382676 & -0.67 & -1.73 \\
\hline $\mathrm{TS}_{2}$ & -548.35589262 & 0.01198362 & +21.77 & +20.04 \\
\hline $\mathrm{O} \cdots \mathrm{HSO}$ & -548.37507429 & 0.01337365 & -11.16 & +8.88 \\
\hline $\mathrm{O}+\mathrm{HSO}$ & -548.36569340 & 0.00984816 & +3.67 & +12.55 \\
\hline
\end{tabular}

O mínimo global deste sistema molecular é a estrutura cis-HOSO s. Muitos trabalhos teóricos e experimentais, já citados, foram dedicados ao estudo desta estrutura. Em particular, dois trabalhos teóricos recentes ${ }^{[136,138]}$ realizaram uma análise bem extensa desta estrutura. A geometria e frequências determinadas no presente trabalho estão em excelente concordância com os resultados reportados nessas referências. A maior diferença aparece no valor do ângulo diedro $\mathrm{H}-\mathrm{O}-\mathrm{S}-\mathrm{O}$ de $54.74^{\circ}$, um pouco diferente do valor obtido na Ref. ${ }^{[136]}$ de $24.20^{\circ}$.

Segundo os resultados mostrados na tabela 5.2, a energia do mínimo global cis$\mathrm{HOSO}_{\mathrm{s}}$ fica 41.63, 72.51 e $102.64 \mathrm{kcal} / \mathrm{mol}$ abaixo dos respectivos limites de dissociação $\mathrm{H}+\mathrm{SO}_{2}, \mathrm{OH}+\mathrm{SO}$ e $\mathrm{SH}+\mathrm{O}_{2}$. Esses valores estão em muito boa concordância 
com os valores $44.24,71.85$ e $96.01 \mathrm{kcal} / \mathrm{mol}$ reportados na Ref. ${ }^{[98]}$ e também com o valor $69.10 \mathrm{kcal} / \mathrm{mol}$ relativo ao limite $\mathrm{OH}+\mathrm{SO}$ da Ref. ${ }^{[138]}$.

Tabela 5.2: Propriedades energéticas dos pontos estacionários da região de baixas energias no diagrama energético.

\begin{tabular}{|c|c|c|c|c|}
\hline $\begin{array}{c}\text { Pontos } \\
\text { estacionários }\end{array}$ & $\begin{array}{l}\text { Energia total } \\
\text { (au) }\end{array}$ & ZPE (au) & $\begin{array}{c}\text { Diferença com a } \\
\text { estrutura anterior } \\
(\mathrm{kcal} / \mathrm{mol})\end{array}$ & $\begin{array}{c}\text { Diferença com o } \\
\text { limite } \mathrm{SH}+\mathrm{O}_{2} \\
(\mathrm{kcal} / \mathrm{mol})\end{array}$ \\
\hline \multicolumn{5}{|c|}{ Caminho de reação $\mathrm{HS}+\mathrm{O}_{2} \rightarrow \mathrm{OH}+\mathrm{SO}$} \\
\hline $\mathrm{HS}+\mathrm{O}_{2}$ & -548.38457423 & 0.00872692 & 0.0 & 0.0 \\
\hline trans-HSOO & -548.39091723 & 0.01332704 & -1.09 & -1.09 \\
\hline $\mathrm{TS}_{1}$ & -548.39088689 & 0.01335814 & +0.04 & -1.06 \\
\hline $\mathrm{HSOO}_{\mathrm{s}}$ & -548.39242944 & 0.01382676 & -0.67 & -1.73 \\
\hline $\mathrm{TS}_{3}$ & -548.36518342 & 0.01328699 & +16.76 & +15.03 \\
\hline $\mathrm{HSO}_{2}(\mathrm{~b})$ & -548.38064416 & 0.01855706 & -6.40 & +8.64 \\
\hline $\mathrm{TS}_{4}$ & -548.37262674 & 0.01330662 & +1.74 & +10.37 \\
\hline $\mathrm{HSO}_{2}$ & -548.52165409 & 0.01568766 & -92.02 & -81.65 \\
\hline $\mathrm{TS}_{5}$ & -548.45949288 & 0.01195595 & +36.67 & -44.99 \\
\hline cis-HOSO & -548.55599064 & 0.01658146 & -57.65 & -102.64 \\
\hline $\mathrm{OH}+{ }^{3} \mathrm{SO}$ & -548.4348512 & 0.01098560 & +72.51 & -30.13 \\
\hline \multicolumn{5}{|c|}{ Caminho de reação (1) $\mathrm{HS}+\mathrm{O}_{2} \rightarrow \mathrm{H}+\mathrm{SO}_{2}$} \\
\hline cis-HOSO & -548.55599064 & 0.01658146 & -57.65 & -102.64 \\
\hline $\mathrm{TS}_{6}$ & -548.46125675 & 0.00814027 & +54.15 & -48.49 \\
\hline $\mathrm{H} \cdots \mathrm{SO}_{2}$ & -548.48006774 & 0.00673368 & -12.69 & -61.17 \\
\hline $\mathrm{H}+\mathrm{SO}_{2}$ & -548.47980364 & 0.00673750 & +0.17 & -61.01 \\
\hline \multicolumn{5}{|c|}{ Caminho de reação (2) $\mathrm{HS}+\mathrm{O}_{2} \rightarrow \mathrm{H}+\mathrm{SO}_{2}$} \\
\hline $\mathrm{HSO}_{2}$ & -548.52165409 & 0.01568766 & -92.02 & -81.65 \\
\hline $\mathrm{TS}_{7}$ & -548.47928308 & 0.00777670 & +21.62 & -60.03 \\
\hline $\mathrm{H} \cdots \mathrm{SO}_{2}$ & -548.48006774 & 0.00673368 & -1.15 & -61.17 \\
\hline $\mathrm{H}+\mathrm{SO}_{2}$ & -548.47980364 & 0.00673750 & +0.17 & -61.01 \\
\hline
\end{tabular}

(1) e (2) indica as duas possíveis formas de chegar ao limite $\mathrm{H}+\mathrm{SO}_{2}$

No nível de cálculo adotado neste trabalho, foram encontrados dois caminhos de reação que permitem a formação de $\mathrm{H}+\mathrm{SO}_{2}$ a partir dos reagentes $\mathrm{OH}+\mathrm{SO}$ (ver figura 5.1). Nos dois casos a dissociação é precedida pelo mínimo de van der Waals $\mathrm{H}^{\cdots} \mathrm{SO}_{2}$, energeticamente separado da dissociação por $0.2 \mathrm{kcal} / \mathrm{mol}$. No primeiro caminho o $\mathrm{TS}_{6}$ aparece para afastar o átomo de hidrogênio do triátomo O-S-O (ver direção da seta na 
figura 5.4) ligando o mínimo global ao complexo de van der Waals. No segundo caminho o $\mathrm{TS}_{7}$ quebra a ligação $\mathrm{H}-\mathrm{S}$ da estrutura $\mathrm{HSO}_{2}$ para formar o mínimo de van der Waals. Esses dois mecanismos são exoenergéticos e garantem a formação de $\mathrm{H}+\mathrm{SO}_{2}$ a partir de $\mathrm{OH}+\mathrm{SO}$.

Os estados de transição $\mathrm{TS}_{5}$ e $\mathrm{TS}_{6}$ concordam muito bem com os estados de transição equivalentes da SEP DMBE ${ }^{[98]}$, apresentando muito poucas diferenças geométricas e energéticas.

$\mathrm{Na}$ literatura está reportado um diagrama energético do sistema $\mathrm{HSO}_{2}$ obtido da teoria DFT $\left(B 3 L Y P / 6-31+G(d){ }^{[131]}\right.$ muito similar à região de baixas energias do diagrama da figura 5.1, com a diferença que não é incluído o mínimo de van der Waals $\mathrm{H}^{\cdots} \cdot \mathrm{SO}_{2}$.

\subsubsection{Caminhos de reação $\mathrm{SH}+\mathrm{O}_{2} \rightarrow \mathrm{OH}+\mathrm{SO}$ (região de maiores energias)}

O diagrama energético mostrado na figura 5.2 representa o caminho de reação para $\mathrm{SH}+\mathrm{O}_{2} \rightarrow \mathrm{OH}+\mathrm{SO}$ apresentado anteriormente, mas incluindo outras estruturas de energias relativamente maiores do que as já apresentadas.

É interessante notar que neste caso a aproximação dos diátomos é em forma planar com os eixos moleculares quase paralelos e dessa forma, o sistema afronta uma barreira energética relativamente alta. No caso da seção anterior, a aproximação dos diátomos acontece com os eixos quase antiparalelos e a formação do complexo inicial é livre de barreira. Na formação da estrutura estacionária cis-HSOO, o sistema passa pelo estado de transição $\mathrm{TS}_{8}$, que apresenta uma altura de $7.33 \mathrm{kcal} / \mathrm{mol}$ em relação ao limite de dissociação $\mathrm{SH}+\mathrm{O}_{2}$ e $8.03 \mathrm{kcal} / \mathrm{mol}$ comparado ao mínimo cis-HSOO. A altura da barreira no sentido da reação está em excelente concordância com o valor de 9.33 $\mathrm{kcal} / \mathrm{mol}$ obtido usando teoria perturbação MP4/6-311G(d,p) ${ }^{[89,94]}$. No sentido inverso, a altura da barreira é menor que o valor reportado no nível multi-reference MP2 $(9,10)$ ${ }^{[137]}$ de $12.94 \mathrm{kcal} / \mathrm{mol}$.

A estrutura cis-HSOO já foi reportada na literatura ${ }^{[138]}$ praticamente com as mesmas propriedades geométricas que as mostradas na figura 5.4. No presente trabalho, essa estrutura foi encontrada com uma frequência imaginária muito baixa, $80.91 \mathrm{~cm}^{-1}$, associada à mudança do ângulo diedro H-S-O-O e sua energia é muito próxima à energia da estrutura $\mathrm{HSOO}_{\mathrm{s}}$ (da ordem de $1 \mathrm{kcal} / \mathrm{mol}$ de diferença). Segundo esses 
resultados, consideramos que a isomerização entre $\mathrm{HSOO}_{\mathrm{s}}$ e sua imagem especular passando pela estrutura cis-HSOO é um processo praticamente sem barreira. A pequena diferença energética entre as estruturas cis-HSOO e $\mathrm{HSOO}_{\mathrm{s}}$, e portanto, a livre isomerização entre elas, permite conectar os estados de transição $\mathrm{TS}_{8}$ e $\mathrm{TS}_{9}$ através de cis-HSOO.

$\mathrm{O}$ estado de transição $\mathrm{TS}_{9}$ da figura 5.2 , com simetria $C_{\mathrm{s}}$, conecta as estruturas estacionárias cis-HSOO e cis-HOOS. A diferença energética de $\mathrm{TS}_{9}$ em relação ao primeiro mínimo é de $20.71 \mathrm{kcal} / \mathrm{mol}$ e $39.20 \mathrm{kcal} / \mathrm{mol}$ em relação ao segundo. Goumri et al. ${ }^{[94]}$ não conseguiram localizar um estado de transição que permitisse a isomerização HOOS $\rightarrow$ HSOO realizando cálculos no nível MP2/6-31G(d). Na SEP DMBE de Ballester e Varandas ${ }^{[98]}$, não é reportado um caminho de reação para a reação $\mathrm{SH}+\mathrm{O}_{2} \rightarrow \mathrm{OH}+\mathrm{SO}$. Entretanto, Resende e Ornellas ${ }^{[137]}$ reportam um estado de transição (equivalente ao $\mathrm{TS}_{9}$ ) que liga diretamente a estrutura $\mathrm{HSOO}_{\mathrm{s}}$ (ou cis-HSOO) com os produtos de reação $\mathrm{OH}+\mathrm{SO}$. A altura da barreira, reportada por eles no nível $\mathrm{CCSD}(\mathrm{T}) / \mathrm{CBS}$, é de $37.19 \mathrm{kcal} / \mathrm{mol}$ em relação a $\mathrm{HSOO}_{\mathrm{s}}, 16.48 \mathrm{kcal} / \mathrm{mol}$ maior do que no nosso resultado, e fica $54.76 \mathrm{kcal} / \mathrm{mol}$ acima da dissociação $\mathrm{OH}+\mathrm{SO}$, concordando muito bem com nosso resultado de $50.15 \mathrm{kcal} / \mathrm{mol}$ em relação à dissociação.

A figura 5.2 mostra também o caminho de reação além do $\mathrm{TS}_{9}$, formado pelos mínimos cis-HOOS e trans-HOOS (ambos de simetria plana) ligados pelo estado de transição $\mathrm{TS}_{10}$. No final do caminho de reação o $\mathrm{TS}_{11}$ indica o caminho da dissociação $\mathrm{OH}+\mathrm{SO}$. As geometrias dessas estruturas estacionárias concordam muito bem com

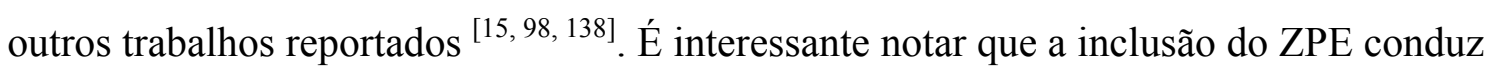
a localizar o $\mathrm{TS}_{10}$ ligeiramente abaixo do mínimo cis-HOOS. Esse resultado indica a necessidade de usar um nível de cálculo superior para descrever melhor essas estruturas estacionárias. As diferenças energéticas, tão pequenas entre essas estruturas (tabela 5.1), sugerem uma rápida transição entre elas, que resultaria em uma fácil dissociação após passar a barreira energética do estado de transição $\mathrm{TS}_{9}$. A mesma conclusão obteve-se nas Refs. ${ }^{[15,137]}$. 
Tabela 5.3: Frequências vibracionais de todas as estruturas estacionárias obtidas.

\begin{tabular}{|c|c|c|c|c|c|c|}
\hline \multirow[b]{2}{*}{$\begin{array}{c}\text { Pontos } \\
\text { estacionários }\end{array}$} & \multicolumn{6}{|c|}{ Frequências $\left(\mathrm{cm}^{-1}\right)$} \\
\hline & f1 & $\mathrm{f} 2$ & $\mathrm{f3}$ & $\mathrm{f} 4$ & f5 & f6 \\
\hline $\mathrm{TS}_{1}$ & $82.84 \mathrm{i}$ & 327.00 & 575.45 & 959.39 & 1236.43 & 2765.28 \\
\hline $\mathrm{TS}_{2}$ & $657.73 i$ & 301.70 & 464.65 & 768.16 & 1093.95 & 2631.74 \\
\hline $\mathrm{TS}_{3}$ & $341.44 \mathrm{i}$ & 459.79 & 712.50 & 899.74 & 1050.18 & 2710.09 \\
\hline $\mathrm{TS}_{4}$ & $1279.05 \mathrm{i}$ & 560.81 & 632.47 & 947.51 & 1048.74 & 2651.40 \\
\hline $\mathrm{TS}_{5}$ & $2931.46 \mathrm{i}$ & 438.51 & 601.10 & 1046.14 & 1304.63 & 1857.67 \\
\hline $\mathrm{TS}_{6}$ & $1854.70 \mathrm{i}$ & 256.88 & 484.20 & 519.12 & 1046.78 & 1266.18 \\
\hline $\mathrm{TS}_{7}$ & $720.05 \mathrm{i}$ & 218.06 & 226.83 & 496.97 & 1126.09 & 1345.63 \\
\hline $\mathrm{TS}_{8}$ & $104.97 \mathrm{i}$ & 82.98 & 248.75 & 517.59 & 2285.84 & 2712.26 \\
\hline $\mathrm{TS}_{9}$ & $2434.64 \mathrm{i}$ & 526.03 & 702.54 & 928.94 & 1738.41 & 1974.47 \\
\hline $\mathrm{TS}_{10}$ & $178.44 \mathrm{i}$ & 409.29 & 693.0 & 866.50 & 1360.65 & 3690.35 \\
\hline $\mathrm{TS}_{11}$ & $481.46 \mathrm{i}$ & 97.75 & 321.14 & 936.09 & 1018.33 & 3701.23 \\
\hline trans-HOSO & $162.80 \mathrm{i}$ & 396.28 & 759.26 & 1058.09 & 1143.27 & 3744.65 \\
\hline cis-HSOO & $-80.9 \mathrm{i}$ & 412.5 & 586.2 & 972.0 & 1258.8 & 2748.3 \\
\hline cis-HOSO & 197.08 & 400.02 & 775.65 & 1059.91 & 1105.38 & 3740.38 \\
\hline $\mathrm{HSO}_{2}$ & 443.78 & 757.15 & 956.33 & 1048.52 & 1265.84 & 2414.47 \\
\hline $\mathrm{HSO}_{2}(\mathrm{~b})$ & 518.14 & 626.30 & 806.94 & 1029.84 & 2487.95 & 2676.44 \\
\hline $\mathrm{HSOO}_{\mathrm{s}}$ & 173.96 & 414.33 & 650.13 & 954.61 & 1130.16 & 2746.05 \\
\hline $\mathrm{H} \cdots \mathrm{SO}_{2}$ & 15.14 & 39.33 & 122.85 & 498.56 & 1119.25 & 1337.94 \\
\hline trans-HSOO & 41.28 & 354.84 & 636.15 & 991.00 & 1244.96 & 2763.36 \\
\hline $\mathrm{O} \cdots \mathrm{HSO}$ & 61.29 & 95.39 & 116.39 & 1077.63 & 2286.16 & 2506.56 \\
\hline cis-HOOS & 178.06 & 411.19 & 708.43 & 888.44 & 1393.45 & 3624.73 \\
\hline trans-HOOS & 95.28 & 388.51 & 652.25 & 862.95 & 1321.36 & 3727.56 \\
\hline
\end{tabular}

\subsubsection{Características gerais dos caminhos de reação mostrados nos diagramas}

A primeira característica interessante é que, a partir dos reagentes $\mathrm{SH}+\mathrm{O}_{2}$, o diagrama energético da figura 5.1 indica que a formação dos produtos de reação $\mathrm{H}+\mathrm{SO}_{2}$ são energeticamente os mais favoráveis. A segunda observação corresponde às barreiras energéticas para obter os produtos de reação $\mathrm{HSO}+\mathrm{O}$ e $\mathrm{OH}+\mathrm{SO}$. Segundo os diagramas das figuras 5.1 e 5.2, as barreiras energéticas para obter estes produtos de reação, dadas pelos estados de transição $\mathrm{TS}_{2}$ e $\mathrm{TS}_{9}$, são equivalentes. Portanto, esses processos são 
energeticamente competitivos. Da análise dos diagramas energéticos, podemos ver também que reações que começam com energia total menor que as energias dos estados de transição $\mathrm{TS}_{2}$ e $\mathrm{TS}_{9}$ e maior que a energia de $\mathrm{TS}_{3}$ vão conduzir principalmente à formação dos produtos de reação $\mathrm{H}+\mathrm{SO}_{2}$. Se a energia total dos reagentes fosse suficiente para passar as barreiras dos estados de transição $\mathrm{TS}_{2}$ e $\mathrm{TS}_{9}$, então a formação dos produtos $\mathrm{HSO}+\mathrm{O}$ e $\mathrm{OH}+\mathrm{SO}$ é favorecida. Este último produto de reação é mais favorável seguindo o caminho da figura 5.2, pois o caminho menos energético mostrado na figura 5.1 requer muito rearranjo geométrico.

\subsection{Conclusões}

É reportado um conjunto de estruturas estacionárias e o diagrama energético da superfície de energia potencial do sistema molecular $\mathrm{HSO}_{2}$ no estado eletrônico fundamental. Todas as estruturas foram otimizadas no nível de cálculo CASPT2/aug-cc$\mathrm{pV}(\mathrm{T}+\mathrm{D}) \mathrm{Z}$ e as energias foram extrapoladas a um conjunto de bases infinito. De forma geral, as estruturas concordam muito bem com resultados anteriormente reportados.

Neste trabalho é reportado um novo caminho de reação que conecta a região de maiores energias da SEP à região menos energética. Esse caminho de reação liga os mínimos $\mathrm{HSOO}_{\mathrm{s}}$ e $\mathrm{HSO}_{2}$ através dos estados de transição $\mathrm{TS}_{3}$ e $\mathrm{TS}_{4}$, passando pela estrutura intermediária $\mathrm{HSO}_{2}(\mathrm{~b})$ e torna possível a reação $\mathrm{SH}+\mathrm{O}_{2} \rightarrow \mathrm{H}+\mathrm{SO}_{2}$. A caracterização do mínimo global cis- $\mathrm{HOSO}_{\mathrm{s}}$ está em excelente concordância com trabalhos anteriores. Segundo os diagramas energéticos elaborados, é possível determinar os intervalos energéticos favoráveis para obter os produtos de reação $\mathrm{H}+\mathrm{SO}_{2}$ e $\mathrm{HSO}+\mathrm{O}$ a partir dos reagentes $\mathrm{HS}+\mathrm{O}_{2}$. Estas características energéticas devem ser verificada experimentalmente ou em estudos teóricos de dinâmica de reação.

Finalmente, a presença de água em fase condensada na troposfera pode influir na reatividade de reações associadas a este sistema molecular. Assim, os resultados obtidos neste trabalho podem ser usados para estudar a solvatação ou microsolvatação dos diferentes caminhos de reação. 
96 Estudo da superfície de energia potencial do sistema molecular $\mathrm{HSO}_{2}$ 


\section{Capítulo 6}

\section{Polarização do estado excitado e}

\section{reavaliação da emissão $n \leftarrow \pi^{*}$ da}

\section{acetona em água}

O espectro de absorção da acetona em água tem sido objeto de numerosos trabalhos teóricos ${ }^{[78,147-154]}$, pois é um dos sistemas favoritos para testar modelos teóricos de solventes. A maioria dos trabalhos teóricos que estudam o efeito de solventes em propriedades moleculares descrevem corretamente o deslocamento da banda de absorção $\mathrm{n} \rightarrow \pi^{*}$ da acetona em água. Na Ref. ${ }^{[152]}$ são apresentados alguns resultados dos trabalhos teóricos mais recentes sobre o espectro de absorção da acetona em água. Porém, o espectro de emissão $\mathrm{n} \leftarrow \pi^{*}$ foi muito menos estudado, e de fato, o deslocamento da banda de emissão provocado pelo solvente ainda é objeto de incerteza.

$\mathrm{Na}$ literatura foram publicados três estudos teóricos do espectro de emissão da acetona. No primeiro deles ${ }^{[151]}$ foram realizados cálculos semiempíricos INDO/CIS nas estruturas soluto-solvente, obtendo-se um deslocamento em água de $1850 \mathrm{~cm}^{-1}$ comparado com a fase gasosa. Na mesma época, num outro estudo usando dinâmica molecular de Car-Parrinello ${ }^{[153]}$ obteve-se um deslocamento espectral consideravelmente menor, da ordem de $100 \mathrm{~cm}^{-1}$, que foi comparado com valores experimentais da ordem dos $2000 \mathrm{~cm}^{-1}$, discordando muito. Recentemente, Öhrn e Karlström ${ }^{[154]}$ usando um método de estatística quântica (QMSTAT), também 
obtiveram que o deslocamento provocado pelo solvente na emissão $\mathrm{n} \leftarrow \pi^{*}$ é quase desprezível. Neste trabalho foi feita uma análise detalhada dos resultados experimentais e concluiu-se que o efeito dos solventes nessa transição deve ser muito pequeno, menor que $0.05 \mathrm{eV}$ (ou $\sim 400 \mathrm{~cm}^{-1}$ ), o que serve de suporte ao resultado do trabalho anterior [153] usando dinâmica de Car-Parrinello. Baseado nesta polemica, e para avaliar a importância da polarização eletrônica no estado excitado da acetona, que não foi considerada no trabalho publicado anteriormente ${ }^{[151]}$, é reconsiderado neste capítulo, o problema da emissão da acetona em água. Usaremos métodos de cálculo além de INDO/CIS e será considerado o efeito da polarização eletrônica da acetona no estado eletrônico excitado. A polarização eletrônica, discutida na seção (3.4), tem sido amplamente estudada no estado fundamental ${ }^{[73-78,151]}$, porém, o efeito dela nos estados eletrônicos excitados tem sido pouco estudado. A análise da polarização eletrônica em estados excitados é particularmente importante no estudo de efeito de solvente na dinâmica de estados excitados ${ }^{[155-157]}$.

A desativação da energia no estado eletrônico excitado pode ocorrer durante a relaxação à estrutura de equilíbrio e, portanto, a emissão pode ser a superposição de diferentes componentes temporais ${ }^{[158]}$. A acetona é um sistema interessante, pois normalmente é considerado que o tempo de vida do estado excitado dela é suficientemente grande e a emissão começa após a relaxação completa da geometria e é possível atingir o equilíbrio eletrostático soluto-solvente. Esse fato é verificado tanto teórico ${ }^{[154]}$ quanto experimentalmente ${ }^{[159]}$. O objetivo do presente trabalho é então fazer uma análise teórica detalhada do papel da polarização eletrônica do estado excitado $n \rightarrow \pi^{*}$ e a sua influência na emissão da acetona em água.

\subsection{Detalhes computacionais}

Neste trabalho foi usado o procedimento sequencial QM/MM ${ }^{[160]}$ descrito no capítulo (3), onde na simulação de MC são geradas as configurações soluto-solvente energeticamente descorrelacionadas a serem usadas nos cálculos de mecânica quântica dos espectros de emissão e absorção. As simulações de MC foram realizadas usando o código DICE ${ }^{[69]}$ no ensemble NPT em condições normais de temperatura e pressão $(T=$ $\left.25^{\circ} \mathrm{C}, P=1 \mathrm{~atm}\right)$. Foram consideradas 450 moléculas de água numa caixa cúbica com condições periódicas de contorno ${ }^{[68]}$. O potencial de interação intermolecular é dado 
pela equação (3.8), onde para as moléculas de água foram usados os parâmetros de Lennard-Jones e cargas do modelo TIP3P (three-site transferrable intermolecular potential) ${ }^{[161]}$. Para o estado eletrônico fundamental e primeiro estado excitado da acetona os valores $\varepsilon_{i}$ e $\sigma_{i}$ foram extraídos de Gao et al. ${ }^{[150]}$. As cargas dos sítios atômicos da acetona, no estado eletronico fundamental e excitado, foram obtidas da polarização eletrônica de cada um destes estados seguindo o procedimento iterativo descrito na seção (3.4).

Tabela 6.1: Parâmetros usados no potencial de Lennard-Jones. Também são mostrados os momentos de dipolo e cargas convergidos no procedimento iterativo. Os índices atômicos são mostrados na figura 6.1 .

\begin{tabular}{ccccc}
\hline Átomo & $\varepsilon_{i}(\mathrm{kcal} / \mathrm{mol})$ & $\sigma_{i}(\AA)$ & \multicolumn{2}{c}{$q_{i}(e)$} \\
\hline & & & Fund. & Exc. \\
$\mathrm{O}$ & 0.210 & 2.960 & -0.7109 & -0.2091 \\
$\mathrm{C}_{1}$ & 0.105 & 3.750 & 0.8133 & -0.1939 \\
$\mathrm{C}_{2}$ & 0.160 & 3.910 & -0.4216 & 0.1032 \\
$\mathrm{H}_{1}$ & 0.000 & 0.000 & 0.1124 & 0.0219 \\
$\mathrm{H}_{2}$ & 0.000 & 0.000 & 0.1352 & 0.0517 \\
$\mathrm{H}_{3}$ & 0.000 & 0.000 & 0.1228 & 0.0247 \\
\hline & & & Momento de dipolo (D) \\
Presente trabalho & & & $4.80 \pm 0.03$ & $3.00 \pm 0.03$ \\
Ref. ${ }^{[153]}$ & & & $4.90 \pm 0.05$ & $3.10 \pm 0.05$ \\
\hline
\end{tabular}

Nas simulações realizadas nos procedimentos iterativos foram calculados $1.5 \times 10^{5}$ passos de MC no processo de termalização, seguido de $3.0 \times 10^{5}$ na geração de configurações soluto-solvente. Em cada simulação foram selecionadas 100 configurações soluto-solvente com menos de $12 \%$ de correlação estatística ${ }^{[162]}$ para determinar os momentos de dipolo e cargas do soluto. Estas magnitudes foram calculadas no nível MP2/aug-cc-pVTZ para o estado fundamental e CIS/aug-cc-pVTZ para o estado eletrônico excitado. No cálculo das cargas foi usado o mapeamento eletrostático CHELPG ${ }^{[79]}$ A geometria de equilíbrio da acetona no estado excitado obteve-se de Liao et al. ${ }^{[163]}$, determinada no nível $\operatorname{CASSCF}(10,11) / 6-311++\mathrm{G}(\mathrm{d})$. Enquanto, a otimização de geometria do estado eletrônico fundamental foi calculada no nível MP2/aug-cc-pVDZ. 

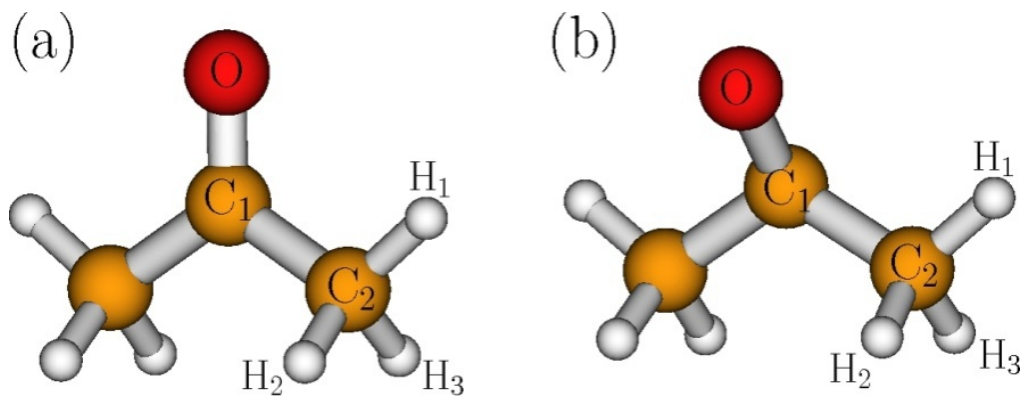

Figura 6.1: Representação geométrica da acetona no estado eletrônico fundamental (a) e o primeiro estado eletrônico excitado (b).

Os cálculos dos espectros de absorção e emissão, foram realizados usando as estruturas soluto-solvente energeticamente descorrelacionadas obtidas das simulações de Monte Carlo, depois de ser atingido o equilíbrio eletrostático soluto-solvente no procedimento iterativo. Estes espectros foram calculados das duas formas seguintes; usando o modelo discreto, onde as moléculas de solvente são consideradas unicamente como cargas pontuais usando o procedimento ASEC ${ }^{[80]}$, e usando o modelo explícito, onde algumas moléculas de água são consideradas explicitamente. Os cálculos foram realizados usando o pacote de programas Gaussian $03^{[164]}$ no nível CIS(D) ${ }^{[27]}$, com os conjuntos de funções-base aug-cc-pVDZ e aug-cc-pVTZ ${ }^{[165]}$ segundo o caso.

\subsection{Resultados e discussão}

\subsubsection{Polarização do soluto no estado eletronico fundamental e primeiro estado excitado}

Antes de descrever a polarização eletrônica da acetona no estado eletrônico fundamental e no primeiro estado excitado, será feita uma descrição dos momentos de dipolo na fase gasosa. Para isso foram usadas as geometrias de equilíbrio otimizadas para os estados eletrônicos fundamental e primeiro excitado, mostradas na figura 6.1. Como é conhecido, o deslocamento para o azul na transição de absorção pode ser entendido em termos dos momentos de dipolo. A figura 6.2 mostra os momentos de dipolo calculados na fase gasosa. Na configuração de equilíbrio do estado eletrônico fundamental o momento de dipolo calculado é $2.98 \mathrm{D}$, em muito boa concordância com o resultado 
experimental de $2.93 \mathrm{D}^{[166]}$. Na transição vertical de absorção acontece um decréscimo considerável no momento de dipolo, indo para 1.76 D. Esse decréscimo está relacionado com o deslocamento espectral para o azul, pois indica que o estado eletrônico excitado solvata menos que o estado fundamental, aumentando assim a separação entre os estados eletrônicos. Na transição de emissão temos que levar em consideração a geometria de equilíbrio do estado excitado. O elemento mais relevante na relaxação desse estado é a saída do plano dos átomos O-C-C-C (figura 6.1). Após a relaxação da geometria, o momento de dipolo no estado excitado aumenta de 1.76 para 2.08 D. Logo depois da transição vertical de emissão para o estado fundamental, o momento de dipolo aumenta para 2.93 D. Portanto, o estado fundamental na geometria de equilíbrio do estado excitado apresenta um momento de dipolo ligeiramente menor que na sua geometria de equilíbrio. Esse esquema é similar ao obtido por Öhrn e Karlström ${ }^{[154]}$.

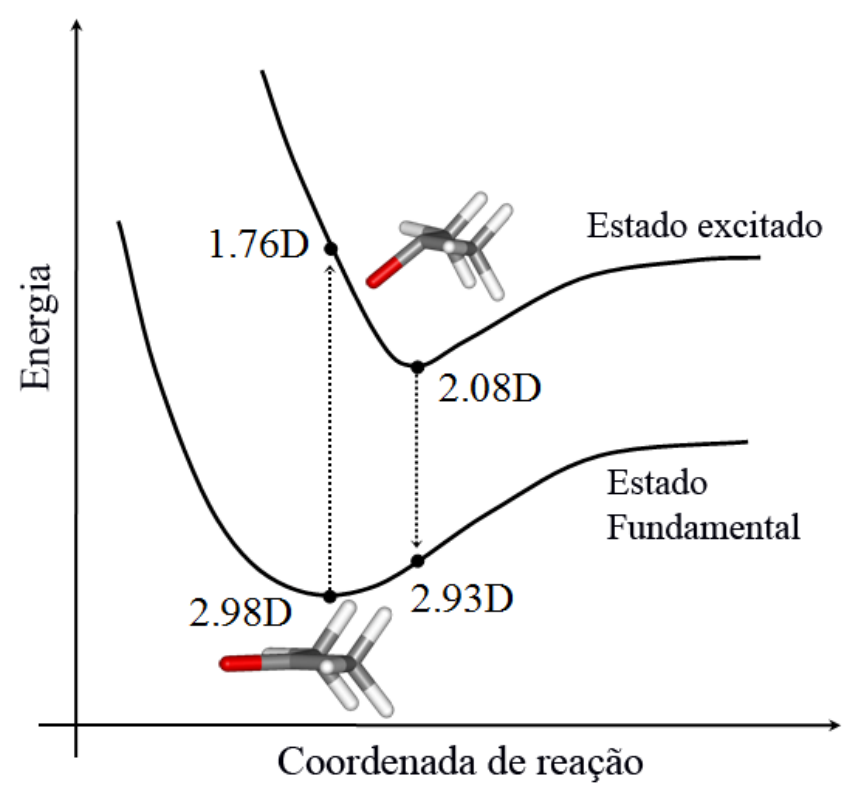

Figura 6.2: Momentos de dipolo calculados nos pontos iniciais e finais das transições verticais em fase gasosa.

A seguir, será analisada a polarização eletrônica da acetona em água. Na figura 6.3 é mostrado o procedimento iterativo que conduz ao equilíbrio eletrostático solutosolvente em cada um dos estados eletrônicos. O momento de dipolo do estado excitado aumenta quase $50 \%$, indo de $2.08 \mathrm{D}$ em fase gasosa para $3.00 \mathrm{D}$ em água. Este valor do dipolo, em equilíbrio com o solvente, está em excelente concordância com o momento de dipolo obtido da dinâmica molecular de Car-Parrinello ${ }^{[153]}$ de 3.08 D. No estado 
eletrônico fundamental obteve-se uma mudança de $2.98 \mathrm{D}$ em fase gasosa para 4.80 D em água. O valor obtido da dinâmica molecular de Car-Parrinello ${ }^{[153]}$ foi de $4.90 \mathrm{D}$, em excelente concordância com nosso resultado. Estes valores são resumidos na tabela 6.1.

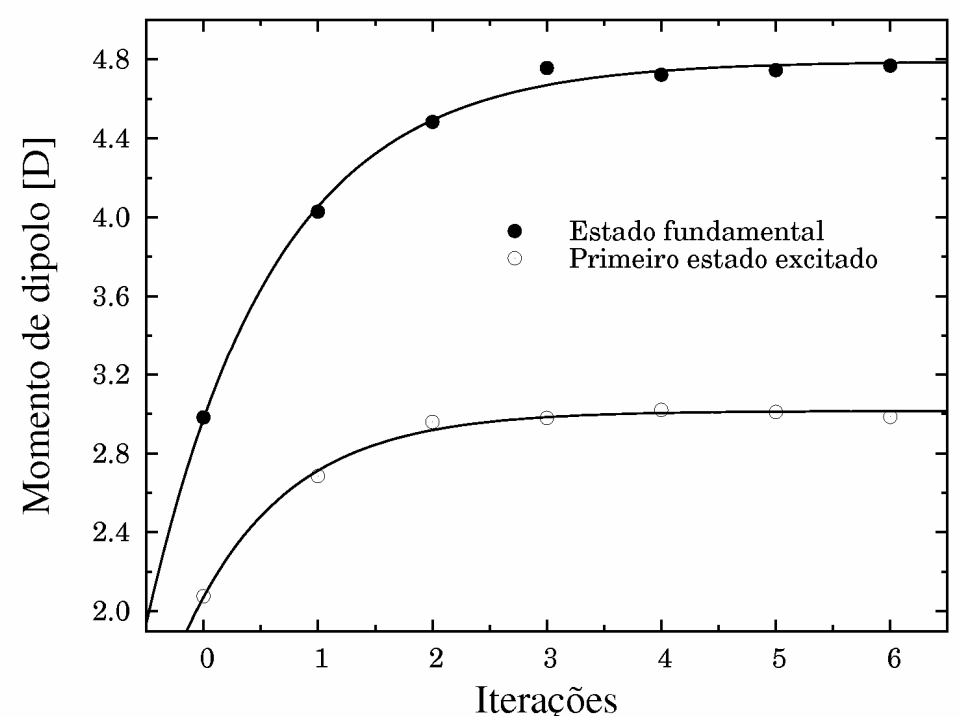

Figura 6.3: Valores médios dos momentos de dipolo nos estados eletrônicos fundamental e excitado em relação ao número de iterações.

Os valores médios convergidos das cargas dos sítios atômicos do soluto na presença do solvente são também mostrados na tabela 6.1. Estas cargas são usadas nas simulações de MC de onde são geradas as configurações soluto-solvente a serem usadas nos cálculos dos espectros de absorção e emissão. Comparando as cargas do estado eletrônico fundamental com as cargas do estado excitado, a mudança mais significativa aparece no átomo de oxigênio, cujo decréscimo conduz à diminuição das pontes de hidrogênio entre a acetona e a água. De fato, usando a função de distribuição radial entre o átomo de oxigênio da acetona e um dos átomos de hidrogênio da água, foi determinado o número de coordenação, igual a 2.5 para o estado eletrônico fundamental e 1.6 para o estado excitado. Estes valores estão em excelente concordância com os obtidos da dinâmica molecular de Car-Parrinello ${ }^{[153]}$, iguais a 2.4 e 1.6 respectivamente. Porém, para obter precisamente o número de ligações de hidrogênio foi utilizado um critério geométrico e energético já usado anteriormente ${ }^{[151,167]}$ e obteve-se um valor médio de 2.1 pontes de hidrogênio entre a acetona e a água no estado eletrônico fundamental e 0.6 no estado excitado. 


\subsubsection{Efeito de solvente na emissão $n \leftarrow \pi *$}

Depois de ter analisado detalhadamente a polarização eletrônica do soluto, foi estudado o espectro de emissão da acetona em água. Como complemento, também foi estudada a transição de absorção. No modelo discreto, como foi comentado, as moléculas de solvente são consideradas unicamente como cargas pontuais. No modelo explícito, 12 moléculas de água são consideradas explicitamente, embebidas no campo eletrostático gerado por 263 moléculas de água representadas como cargas pontuais. A primeira camada de solvatação é formada por aproximadamente 22 moléculas de água. Mas, foram selecionadas as 12 moléculas mais próximas ao soluto, estabelecendo um equilíbrio entre custo computacional e número de moléculas explícitas. A figura 6.4 mostra uma imagem representativa da acetona com as 12 moléculas de água mais próximas e o resto do solvente representado como cargas pontuais.
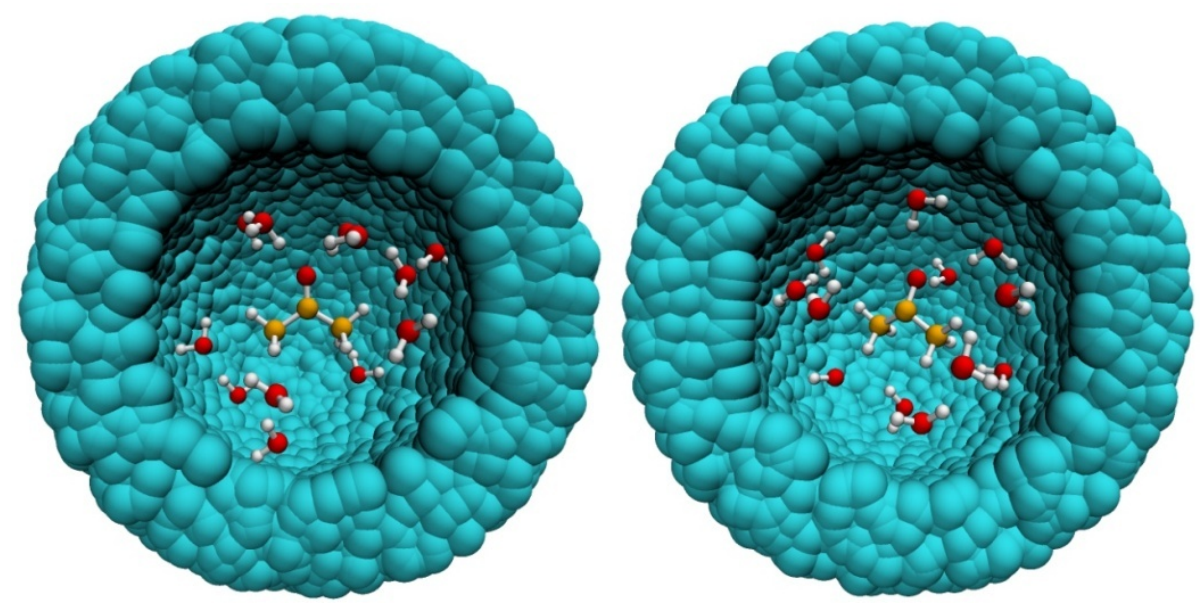

Figura 6.4: Ilustração das configurações soluto-solvente considerando 12 moléculas de água explicitamente e 263 como cargas pontuais. A figura da esquerda corresponde ao estado eletrônico fundamental e da direita ao primeiro estado eletrônico excitado. Os cálculos dos espectros foram feitos em 55 dessas configurações.

Existem vários trabalhos teóricos sobre o efeito de solvente no espectro de absorção da acetona, um sumário deles é apresentado na tabela 7 da Ref. ${ }^{[152]}$. Na tabela 6.2 são apresentados os nossos resultados obtidos neste trabalho e os estudos recentes mais relevantes que consideram tanto a absorção quanto a emissão. Nosso melhor resultado é obtido com o modelo de solvente explícito, calculado no nível CIS(D)/aug-cc-pVDZ. 
Este resultado apresenta uma transição de absorção em água de $4.70 \mathrm{eV}$, deslocado para o azul $0.23 \mathrm{eV}\left(1820 \mathrm{~cm}^{-1}\right)$ em comparação com a fase gasosa. A convergência estatística é mostrada na Figura 6.5. Este valor concorda muito bem com o resultado experimental de um deslocamento para o azul de entre 1500 e $1700 \mathrm{~cm}^{-1}$. Esse resultado também se compara muito bem com o resultado anterior ${ }^{[78]} \mathrm{de} 1650 \mathrm{~cm}^{-1}$ usando o método semiempírico INDO/CIS.

É sabido que o aumento do comprimento da ligação CO, causado pelas pontes de hidrogênio com a água, provoca pequenos deslocamentos espectrais para o vermelho $[152,168]$. Assim, considerando a geometria da acetona em água no estado fundamental dever-se-ia esperar um decréscimo de $\sim 200 \mathrm{~cm}^{-1}$ no deslocamento espectral de absorção. Isto produziria, aproximadamente, um deslocamento espectral para o azul entre 1600 e $1650 \mathrm{~cm}^{-1}$, que concorda ainda melhor com os resultados experimentais. Este raciocínio não se aplica ao estado excitado, pois, como já foi comentado, apresenta uma considerável diminuição da quantidade de ligações de hidrogênio e o alongamento da ligação CO é praticamente desprezível.

Tabela 6.2: Energias de absorção calculadas (eV) em fase gasosa e em água e comparação com trabalhos teóricos e experimentais anteriores.

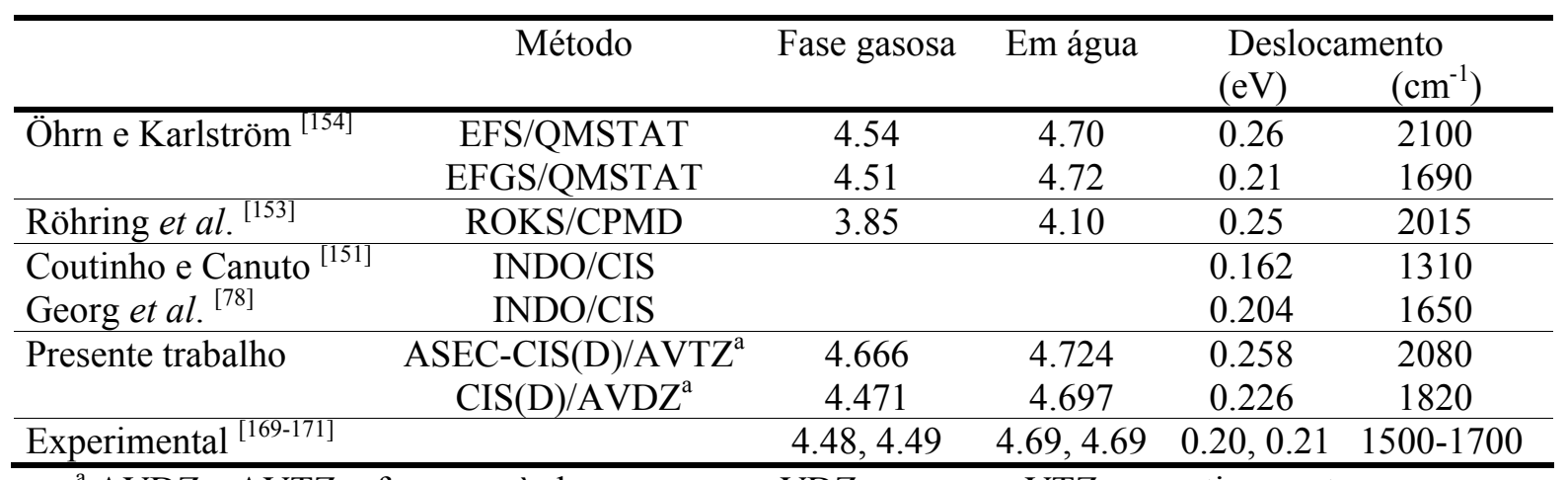

${ }^{\mathrm{a}}$ AVDZ e AVTZ referem-se às bases aug-cc-pVDZ e aug-cc-pVTZ respectivamente.

Voltando ao estudo do espectro de emissão, temos que o deslocamento espectral da banda de emissão obtido por Öhrn e Karlström ${ }^{[154]}$ está entre 160 e $400 \mathrm{~cm}^{-1}$. (Por conveniência e melhor comparação com outros resultados reportaremos o deslocamento espectral $\mathrm{cm}^{-1}$ ). Em concordância com esse resultado, no estudo ROKS Car-Parrinello ${ }^{[153]}$ obteve-se um deslocamento bastante pequeno de $100 \mathrm{~cm}^{-1}$. Anteriormente, tinha sido obtido na Ref. ${ }^{[151]}$ um deslocamento espectral muito grande, da ordem de 1850 $\mathrm{cm}^{-1}$. Esse resultado poderia ser provocado por deficiências do método semiempírico na descrição do solvente, ou por não considerar a polarização eletrônica no estado 
eletrônico excitado da acetona. Mas, também foi encontrada uma falha nos parâmetros do campo de força usado, sendo mais adequados para o estado eletrônico fundamental. Os resultados atuais do espectro de emissão da acetona em água são mostrados na tabela 6.3. Podemos ver que mesmo no modelo discreto (ASEC), a energia de emissão de 350 $\mathrm{cm}^{-1}$ é muito boa, sendo comparável com os outros resultados. Nos cálculos usando o modelo explícito foi usado um conjunto de funções-base ligeiramente menor, realizando os cálculos no nível CIS(D)/aug-cc-pVDZ. O resultado estatisticamente convergido, mostrado na figura 6.5, indica uma energia de emissão de $2.445 \mathrm{eV}$, correspondendo a um deslocamento para o azul de $380 \mathrm{~cm}^{-1}$ comparado com a fase gasosa. Isto corrobora os resultados e interpretações de Öhrn e Karlström de pouca influência do solvente na emissão da acetona. Consideremos agora os resultados semiempíricos mostrados na tabela 6.3. Pode-se ver que neste caso o método INDO/CIS pode oferecer uma correta descrição do efeito do solvente no processo de emissão. Embora o deslocamento obtido seja um pouco maior que com os outros métodos teóricos, $600 \mathrm{~cm}^{-1}$ ainda pode ser considerado um bom resultado.

Tabela 6.3: Energias de emissão (eV) calculadas em fase gasosa e em água e comparação com resultados teóricos e experimentais anteriores. Os resultados apresentados têm um erro estatístico de $\sim 60 \mathrm{~cm}^{-1}$.

\begin{tabular}{|c|c|c|c|c|c|}
\hline \multirow{3}{*}{$\begin{array}{c}\text { Ref. } \\
\text { Öhrn e Karlström } \\
{[154]}\end{array}$} & \multirow{2}{*}{ Método } & \multirow{2}{*}{ Fase gasosa } & \multirow{2}{*}{ Em água } & \multicolumn{2}{|c|}{ Deslocamento } \\
\hline & & & & $(\mathrm{eV})$ & $\left(\mathrm{cm}^{-1}\right)$ \\
\hline & EFS/QMSTAT & 2.07 & 2.12 & 0.05 & 400 \\
\hline & EFGS/QMSTAT & 2.05 & 2.07 & 0.02 & 160 \\
\hline Röhring et al. ${ }^{[153]}$ & ROKS/CPMD & 2.46 & 2.45 & -0.01 & -80 \\
\hline \multirow[t]{4}{*}{ Presente trabalho } & CIS(D)/AVDZ ${ }^{a}$ & 2.398 & 2.408 & 0.010 & 80 \\
\hline & ASEC-CIS(D)/AVTZ ${ }^{b}$ & 2.412 & 2.456 & 0.044 & 350 \\
\hline & CIS(D)/AVDZ ${ }^{\mathrm{b}}$ & 2.398 & 2.445 & 0.047 & 380 \\
\hline & $\mathrm{INDO}^{-\mathrm{CIS}}{ }^{\mathrm{b}}$ & 2.323 & 2.398 & 0.075 & 600 \\
\hline Experimento $^{c)}$ & & 3.01 & $3.00,3.06$ & & \\
\hline
\end{tabular}

${ }^{a}$ Primeiro passo da polarização eletrônica, correspondendo ao momento de dipolo do estado excitado de $2.7 \mathrm{D}$.

${ }^{\mathrm{b}}$ Polarização eletrônica convergida, correspondendo ao momento de dipolo do estado excitado de $3.0 \mathrm{D}$.

${ }^{\mathrm{c}}$ Resultados experimentais em gás ${ }^{[172]}$ e em diferentes solventes ${ }^{[159,173,174]}$.

Para fazer mais explícito o papel da polarização eletrônica, foi feito o cálculo do espectro de emissão nas configurações soluto-solvente obtidas do primeiro ponto do procedimento iterativo, onde a distribuição eletrônica do soluto ainda não está 
completamente polarizada. Neste caso, o momento de dipolo da acetona no estado eletrônico excitado é $2.7 \mathrm{D}$, comparado com o valor convergido de $3.0 \mathrm{D}$. Usando o modelo explícito no nível CIS(D)/aug-cc-pVDZ foi calculado um deslocamento para o azul de aproximadamente $80 \mathrm{~cm}^{-1}$. Esse resultado concorda muito bem com o resultado da dinâmica de Car-Parrinello de Röhring et al. ${ }^{[153]}$, especialmente se é levado em consideração o erro estatístico de $60 \mathrm{~cm}^{-1}$. Assim, a diferença do deslocamento espectral entre considerar o soluto não polarizado e completamente polarizado é de apenas 300 $\mathrm{cm}^{-1}$. Este resultado está fortemente relacionado com a pouca mudança que tem o momento de dipolo do estado excitado na polarização eletrônica.
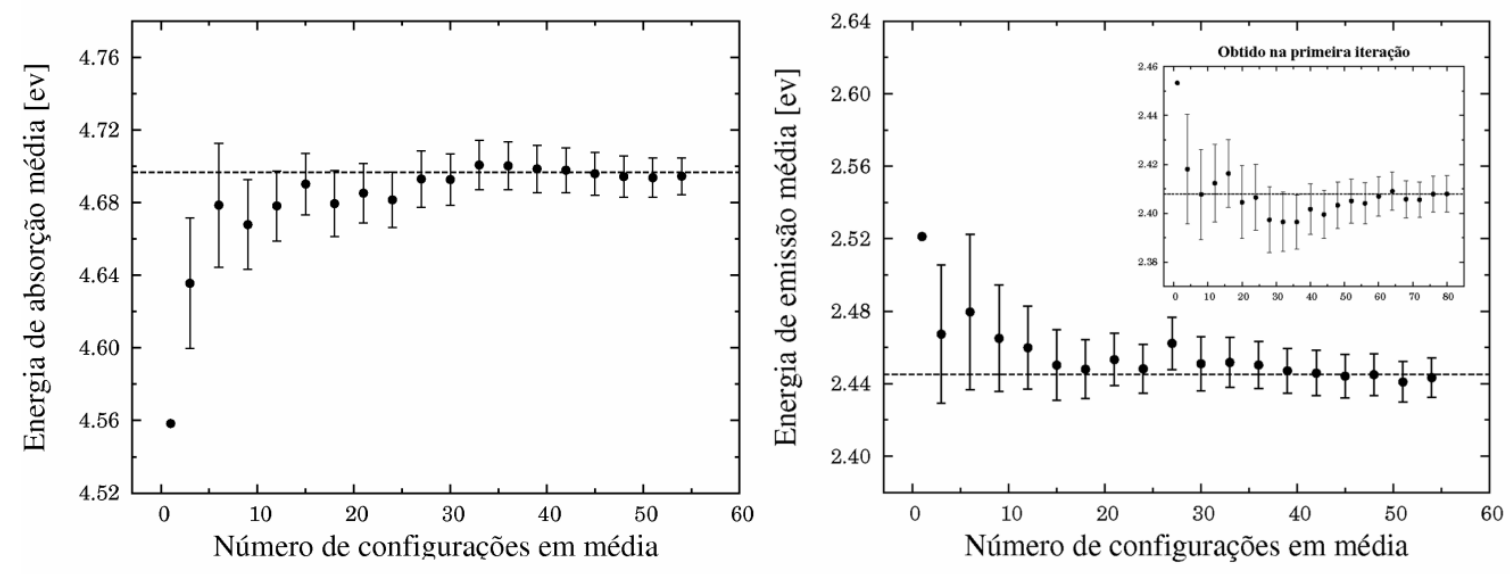

Figure 6.5: Convergência estatística das energias de absorção (esquerda) e emissão (direita) da acetona em água.

\subsection{Conclusões}

É esperado que a polarização eletrônica apresente um papel importante na dinâmica, reatividade e fotoquímica de sistemas moleculares em solução. Neste capítulo foi usada a metodologia sequencial QM/MM para investigar o papel da polarização eletrônica na emissão $\mathrm{n} \leftarrow \pi^{*}$ da acetona em água.

O procedimento iterativo permitiu considerar esse efeito tanto no estado eletrônico fundamental quanto no estado excitado. Os momentos de dipolo médios convergidos concordam muito bem com outros resultados anteriores. Na geometria de equilíbrio do estado eletrônico excitado da acetona, o momento de dipolo calculado em água é de 3.0 
D, concordando muito bem com o resultado de 3.1 D obtido usando dinâmica de CarParrinello ${ }^{[153]}$.

Nosso melhor resultado do deslocamento espectral é obtido no nível de cálculo CIS(D)/aug-cc-pVDZ em 55 configurações soluto-solvente energeticamente descorrelacionadas, obtendo-se resultados estatisticamente convergidos. Cada uma destas configurações foi formada pela acetona e 12 moléculas de água explícitas, embebidas no campo eletrostático de 263 moléculas de água consideradas como cargas pontuais. A análise da emissão $n \leftarrow \pi^{*}$ indica pouca influência do efeito da água, devido à pouca variação do momento de dipolo na polarização eletrônica do estado excitado, sendo que o deslocamento espectral obtido foi de $80 \pm 60$ e $380 \pm 60$, dependendo do nível de polarização eletrônica considerado. 
108 Polarização do estado excitado e reavaliação da emissão da acetona em água 


\section{Capítulo 7}

\section{Estudo teórico da fotofísica do}

\section{sistema molecular 1-nitronaftaleno e}

\section{efeito dos solventes metanol e etanol}

A fotofísica do sistema molecular 1-nitronaftaleno, mostrado na figura 7.1, tem recebido uma atenção particular dos experimentais nos últimos anos. Estudos anteriores em compostos nitroaromáticos têm indicado que a presença do grupo $\mathrm{NO}_{2}$ ligado a compostos aromáticos policíclicos levam o sistema, após a excitação para o primeiro estado eletrônico singleto, a uma rápida transferência desse estado para estados tripletos e finalmente para um estado tripleto fosforescente ${ }^{[175-179]}$. Embora estes compostos tenham sido considerados não fluorescentes, o espectro de emissão deles foi observado através do frequency up-conversion scheme em escalas de tempo de subpicosegundos, apresentando um rápido decaimento da fluorescência ${ }^{[180]}$. Além da formação do estado tripleto fosforescente nestes sistemas, pode existir um caminho de relaxação do estado excitado que leva o sistema à dissociação do grupo $\mathrm{NO}_{2}$ em óxido de nitrogênio $\mathrm{NO} \cdot \mathrm{e}$ um radical aryoxyl Ar-O. ${ }^{[181-183]}$. Na ausência de fotoprodutos de reação, o rápido decaimento da fluorescência pode ser explicado a partir de transições de conversão interna ou intersystem crossing entre estados eletrônicos.

O sistema molecular 1-nitronaftaleno foi estudado junto a outros compostos por Morales-Cueto et al. ${ }^{[180]}$, sendo o 1-nitronaftaleno o único composto que apresenta um 
decaimento em forma exponencial simples. Posteriormente outro estudo experimental [184] indicou que em vários solventes o tempo de vida do estado excitado $\mathrm{S}_{1}$ no 1nitronaftaleno é de 100 fs ou menos, indicando uma rápida mudança de multiplicidade. Desde que não tem sido detectado fotoprodutos de reação ${ }^{[176,178,185,186]}$, essa ultrarápida troca de multiplicidade tem sido atribuída a uma extrema manifestação da regra de El-Sayed ${ }^{[35]}$, permitindo que aconteça um rápido ISC entre o primeiro estado eletrônico excitado e os estados tripletos ${ }^{[184,185]}$.

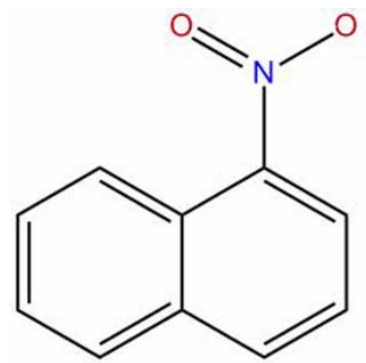

Figura 7.1. Representação do sistema molecular 1-nitronaftaleno.

O 1-nitronaftaleno também foi estudado por Zugazagoitia et al. ${ }^{[187]}$ e Reichardt et al. ${ }^{[188]}$, onde estudos experimentais de espectroscopia de absorção transitória mostram a existência de um estado tripleto que atua como intermediário entre o estado excitado singleto e os estados tripletos. Posteriormente o sistema evolui através de uma CI para o estado tripleto fosforescente. Porém, não existe um consenso definitivo sobre quais são os estados eletrônicos envolvidos nem os mecanismos que permitem a tão eficiente troca de multiplicidade. Portanto, o objetivo do presente trabalho é esclarecer de forma teórica a ultrarápida desativação eletrônica não fluorescente deste sistema molecular, assim como descrever os espectros de absorção transitórias observados.

O desenvolvimento do trabalho foi orientado ao estudo do ISC entre o estado eletrônico excitado singleto e os estados tripletos. A eficiência do mecanismo de ISC requer pequenas diferenças energéticas entre os estados eletrônicos envolvidos, a fim de favorecer o acoplamento vibracional. Além disso, o valor do acoplamento spin-órbita (SOC) entre os estados eletrônicos deve ser consideravelmente grande ${ }^{[189-193]}$, pois, o SOC é o principal responsável pela troca do spin. A busca de condições favoráveis para o ISC no 1-nitronaftaleno é dirigida neste trabalho ao estudo do caminho de relaxação de mínima energia (MEP) do estado excitado singleto, a fim de encontrar regiões de 
degenerescência com os estados tripletos. Ao mesmo tempo o SOC e o ISC rate entre o estado singleto e os estados tripletos serão avaliados.

A descrição correta das bandas de absorção transitórias obtidas experimentalmente

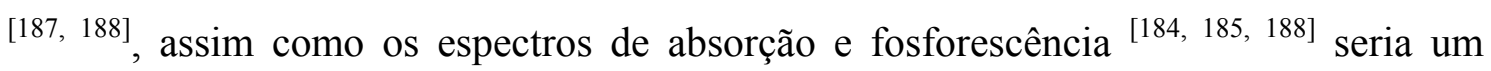
grande suporte para o mecanismo proposto de desativação não radiativa. Por essa razão, foi necessário considerar o efeito de solvente no estado fundamental e nos estados eletrônicos excitados, pois nos experimentos foram usados vários solventes, incluindo metanol e etanol.

O presente estudo está organizado na seguinte forma. Inicialmente será estudada a existência de condições favoráveis para a ocorrência do ISC. Com esse objetivo serão usados métodos multiconfiguracionais. Posteriormente, baseado no mecanismo proposto de desativação não radiativa, serão descritos teoricamente os espectros de absorção transitória obtidos experimentalmente ${ }^{[187,188]}$. Para isso será considerado o efeito de solvente nas transições eletrônica, a fim de obter uma boa comparação com os experimentos. Estas transições eletrônicas serão calculadas usando métodos de interação de configurações.

\subsection{Metodologia}

\subsubsection{Intersystem crossing}

No estudo do ISC as energias dos estados eletrônicos singletos e tripletos foram determinadas utilizando a teoria de perturbação de segunda ordem multiconfiguracional (CASPT2 ${ }^{[26,30]}$, que usa a função de onda de referência CASSCF. No cálculo da função de onda CASSCF foram correlacionados 12 elétrons em 12 orbitais. Essa função de onda de referência é chamada de $\operatorname{CASSCF}(12,12)$. O espaço ativo foi formado pelos orbitais $\pi$ mais alto ocupados além do oxigênio lone-pairs e os seis orbitais $\pi^{*}$ mais baixos desocupados. A escolha destes orbitais foi baseada num cálculo multiconfiguracional que considera um espaço ativo maior, onde somente são permitidas as excitações simples, duplas, triplas e quádruplas, sendo estes orbitais os 
que melhor descrevem os estados eletrônicos singletos e tripletos menos energéticos. Foram usadas as funções-base de valência desdobrada dupla-zeta ANO-L (Atomic Natural Orbitals with Large primitive set) contraídas a C, O, N [4s3p1d]/H [2s1p] ${ }^{[194 \text {, }}$ 195] que considera funções difusas e polarizadas nos átomos não hidrogênicos. Os elétrons dos orbitais $1 s$ dos átomos de oxigênio e carbono foram mantidos congelados na perturbação de segunda ordem. Os cálculos CASSCF usam a aproximação state average, onde só um conjunto de orbitais moleculares é usado para descrever, os dois estados eletrônicos mais baixos singletos ou tripletos $\left(\mathrm{S}_{0}, \mathrm{~S}_{1}\right.$ ou $\left.\mathrm{T}_{1}, \mathrm{~T}_{2}\right)$.

O caminho de relaxação de mínima energia do estado excitado singleto, após a transição de Franck-Condon, foi determinado no nível CASSCF, selecionando dos cálculos anteriores os orbitais ativos que melhor caracterizam o estado eletrônico excitado singleto. Nesse sentido, o MEP foi calculado reduzindo o espaço ativo para quatro elétrons em quatro orbitais, devido ao custo computacional envolvido neste tipo de cálculo. Em cada ponto do MEP calculado foram realizados cálculos CASPT2 usando a função de onda de referência $\operatorname{CASSCF}(12 / 12)$ para descrever de forma correta os estados eletrônicos singletos e tripletos (aproximação CASSCF//CASPT2 ${ }^{[196]}$ ).

Os elementos do acoplamento spin-órbita foram calculados ao longo do MEP usando a aproximação do hamiltoniano efetivo de um elétron ${ }^{[34]}$. Esses cálculos foram realizados usando o programa RASSI (Restricted Active Space State Interaction), que calcula propriedades de interação como força de oscilador e SOC entre estados CASSCF ${ }^{[197]}$. A aproximação do hamiltoniano efetivo de um elétron é considerada factível devido a que o acoplamento spin-órbita é dominado pelas excitações simples $[198,199]$. O acoplamento spin-órbita entre os estados eletrônicos $I$ e $F$ é calculado segundo a Ref. ${ }^{[200]}$ como:

$$
\mathrm{SOC}_{I F}=\sqrt{\sum_{\mathrm{u}}\left|\left\langle T_{I, \mathrm{u}}\left|\hat{H}_{\mathrm{SO}}\right| S_{F}\right\rangle\right|^{2}} \quad \mathrm{u}=\mathrm{x}, \mathrm{y}, \mathrm{z},
$$

onde $\mathrm{u}$ representa cada uma das componentes do estado tripleto. O efeito das correlações dinâmicas é incluído nestes cálculos deslocando os elementos diagonais do hamiltoniano spin-órbita para os valores de energia obtidos nos cálculos CASPT2. O programa RASSI está implementado no pacote de programas MOLCAS $7.6^{[201]}$ que é usado neste trabalho em todos os cálculos multiconfiguracionais. 
O ISC rate entre o estado inicial $I$ e o estado final $F$ é aproximadamente calculado usando a regra de ouro para transições não radiativas ${ }^{[202,203]}$.

$$
k_{I S C}^{I F}=\frac{2 \pi}{\hbar} \operatorname{SOC}_{I F}{ }^{2} f
$$

onde $f$ é um fator relacionado com o acoplamento vibracional entre os estados inicial e final. Ou seja, é a densidade de estados vibracionais no estado tripleto ponderada pelo acoplamento de Franck-Condon. A equação (7.2) tem sido muito usada em reações de transferência de elétrons ${ }^{[202-204]}$, onde no limite de altas temperaturas o fator $f$ obtém-se na seguinte forma ${ }^{[204]}$ :

$$
f=\frac{1}{\sqrt{4 \pi \lambda R T}} \exp \left[-\frac{(\Delta E+\lambda)^{2}}{4 \lambda R T}\right],
$$

sendo $\lambda$ a energia de reorganização de Marcus e $\Delta E$ é a variação de energia vertical entre o estado inicial e o estado final. Em transferência de elétron a energia de reorganização $\lambda$ tem uma componente relacionada às mudanças geométricas intramolecular e uma componente relacionada com a reordenação do solvente produto da transferência [202-204]. No intersystem crossing é esperado que a componente intramolecular seja muito mais significativa que o efeito do solvente. Assim, em primeira aproximação, $\lambda$ corresponde à variação de energia quando o sistema muda da geometria de equilíbrio do estado inicial singleto à geometria de equilíbrio do estado final tripleto.

\subsubsection{Transições eletrônicas e efeito de solvente}

Os cálculos dos espectros de absorção, absorção transitória e fosforescência em solventes foram calculados usando o método extensivo CIS(D) ${ }^{[27]}$. Este método foi comentado na seção (1.4.1) e considera as excitações duplas de forma perturbativa, usando a função de onda de referência obtida da interação de configurações, onde são consideradas unicamente as excitações simples. As funções-base usadas nestes cálculos são as bases de Dunning, aug-cc-pVDZ ${ }^{[165]}$. Este nível de cálculo foi adotado para o 
estudo das transições eletrônicas, pois requer menos esforço computacional comparado com o método CASPT2 e tem oferecido bons resultados no cálculo de espectros de absorção ${ }^{[205,206]}$. As geometrias de equilíbrio dos estados eletrônicos tripletos foram otimizadas no nível CIS/aug-cc-pVDZ. Estas geometrias são necessárias para calcular as energias de absorção das transições eletrônicas originadas nos estados tripletos. A geometria do estado fundamental, usada para descrever a transição de absorção, foi otimizada no nível MP2/aug-cc-pVDZ. Estes cálculos foram realizados usado o pacote de programas Gaussian $03^{[164]}$.

O efeito de solvente foi levado em consideração usando a metodologia sequencial $\mathrm{QM} / \mathrm{MM}^{[160]}$. Este procedimento já foi discutido no capítulo (3) e foi aplicado aqui à solvatação dos estados eletrônicos fundamental e excitados. Neste procedimento foi utilizado o código DICE ${ }^{[69]}$ no ensemble NPT. Nas simulações com metanol, usou-se uma molécula de 1-nitronaftaleno e 500 moléculas de metanol, numa caixa cúbica com condições periódicas de contorno ${ }^{[68]}$ e condições normais de temperatura e pressão, 25 ${ }^{\circ} \mathrm{C}$ e $1 \mathrm{~atm}$ respectivamente. Com etanol foram usadas as mesmas condições anteriores, mas, uma molécula de 1-nitronaftaleno e 350 moléculas de etanol.

O potencial intermolecular usado nas simulações usa os parâmetros de LennardJones para o 1-nitronaftaleno, no estado fundamental e excitados, extraídos dos trabalhos de Jorgensen et al. para os anéis aromáticos ${ }^{[207]}$ e para o grupo $\mathrm{R}^{2} \mathrm{NO}_{2}{ }^{[208]}$. Para o metanol e etanol estes parâmetros e as cargas dos sítios atômicos foram extraídos dos trabalhos de Jorgensen ${ }^{[209]}$ e Martin et al. ${ }^{[210]}$ respectivamente. As cargas $q_{i}$ do 1nitronaftaleno, no estado fundamental e nos estados tripletos, foram obtidas considerando a polarização eletrônica de cada um destes estados. Mas, é analisado em cada uma das transições se a polarização eletrônica é relevante, levando em consideração o tempo de vida do estado eletrônico onde a transição se origina.

A polarização eletrônica do soluto é feita seguindo o procedimento iterativo descrito na seção (3.4) e no trabalho anterior, com a diferença que as cargas usadas na primeira simulação não são obtidas na fase gasosa e sim as obtidas num cálculo do soluto imerso num meio de polarização contínua, $\mathrm{PCM}^{[211]}$. Assim, pode-se obter um valor estimado inicial das cargas do soluto na presença de solvente. No cálculo das cargas é usando o mapeamento eletrostático CHELPG ${ }^{[79]}$, nos níveis HF e CIS para os estados eletrônicos fundamental e excitados respectivamente, com o conjunto de funções-base aug-cc-pVTZ. 
Uma vez atingido o equilíbrio eletrostático soluto-solvente no procedimento iterativo, 100 configurações soluto-solvente, energeticamente descorrelacionadas, são selecionadas das $6.0 \times 10^{5}$ geradas na simulação. Essas configurações são usadas no cálculo de mecânica quântica para determinar os espectros de absorção e/ou emissão dos correspondentes estados eletrônicos. Neste trabalho, as energias de emissão e absorção em solvente foram determinadas unicamente usando o modelo eletrostático ASEC [80] devido ao grande custo computacional de colocar explicitamente as moléculas de solvente neste sistema.

\subsection{Resultados e discussão}

\subsubsection{Mecanismo de desativação proposto}

$\mathrm{Na}$ geometria de equilíbrio do estado fundamental foi calculada a energia de absorção no nível CASPT2 usando a função de onda de referência CASSCF(12/12). Esse cálculo indica dois estados tripletos com energias abaixo do estado singleto $\mathrm{S}_{1}$. As forças de oscilador indicam a transição principal sendo de $S_{0}$ para $S_{1}$. A energia de transição de $3.91 \mathrm{eV}$ está em boa concordância com outros resultados teóricos mostrados nas subseção (7.2.2). O estado excitado $\mathrm{S}_{1}$, segundo nossos cálculos, é principalmente descrito pela transição do orbital HOMO-4 para o LUMO+2. O orbital molecular HOMO-4 é formado por uma componente $\pi$ dos anéis aromáticos e uma componente não ligante nos átomos de oxigênio com os planos nodais perpendiculares com o plano do grupo $\mathrm{NO}_{2}$. $\mathrm{O}$ primeiro estado excitado tripleto $\mathrm{T}_{1}$ tem um caráter $\pi \rightarrow \pi^{*}$ principalmente representado pela transição $\mathrm{HOMO} \rightarrow \mathrm{LUMO}+2$, enquanto que $\mathrm{o}$ segundo estado excitado tripleto é principalmente representado pela transição HOMO$2 \rightarrow$ LUMO. O orbital molecular HOMO-2 é um orbital não ligante localizado nos átomos de oxigênio, mas, com o plano nodal paralelo com o plano do grupo $\mathrm{NO}_{2}$. $\mathrm{Na}$ figura 7.2 são mostradas as configurações espaciais destes orbitais moleculares. 


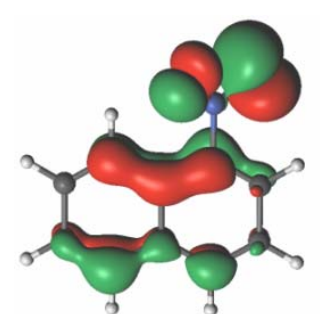

HOMO-4

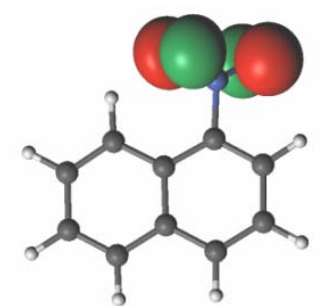

HOMO-2

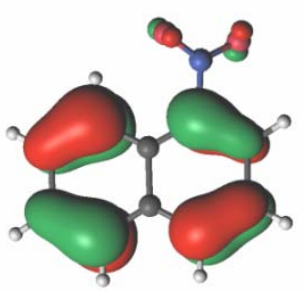

HOMO

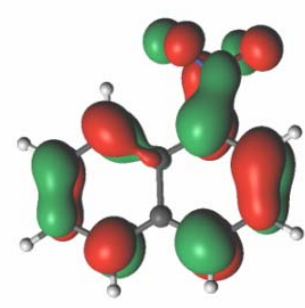

$L U M O$

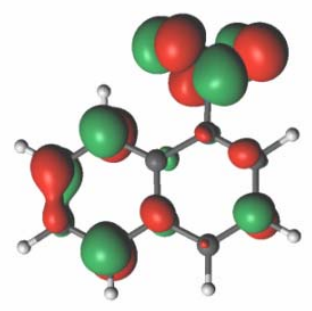

$\mathrm{LUMO+2}$

Figura 7.2. Configurações espaciais dos principais orbitais envolvidos nas transições eletrônicas do estado fundamental para os estados singleto e tripletos.

O caminho de relaxação de mínima energia do estado eletrônico $S_{1}$ após a transição de absorção foi estudado, seguindo a metodologia anteriormente discutida, com o objetivo de encontrar regiões de degenerescência entre o estado eletrônico $S_{1}$ e os estados tripletos. $\mathrm{O}$ acoplamento spin-órbita entre o estado $\mathrm{S}_{1}$ e os estados tripletos mais próximos foram calculados ao longo do MEP de $\mathrm{S}_{1}$. Obtiveram-se valores significativos do SOC da ordem de $65 \mathrm{~cm}^{-1}$ entre os estados $\mathrm{S}_{1}$ e $\mathrm{T}_{2}$. Os valores obtidos com outros estados tripletos são desprezíveis. Na figura 7.3 é mostrado o perfil energético dos estados $\mathrm{S}_{1}$ e $\mathrm{T}_{2}$ calculado no nível CASPT2/ANO-L em cada ponto do MEP do estado $\mathrm{S}_{1}$. Também são mostrados os valores de SOC e ISC rate entre estes estados eletrônicos. Como se pode observar, ao longo do MEP os estados $\mathrm{S}_{1}$ e $\mathrm{T}_{2}$ vão se aproximando um do outro e o SOC mantém um valor consideravelmente grande. $\mathrm{O}$ ponto do MEP correspondente à maior proximidade indica uma diferença energética de $0.11 \mathrm{eV}$. Os valores obtidos do SOC (da ordem dos $65 \mathrm{~cm}^{-1}$ ) são valores consideravelmente grandes comparados com os valores obtidos normalmente para moléculas orgânicas de entre $1 \mathrm{a} 40 \mathrm{~cm}^{-1}{ }^{[212]}$. Nós atribuímos o grande valor do SOC no 1-nitronaftaleno à perpendicularidade entre os planos nodais dos orbitais HOMO-4 e HOMO-2 do grupo $\mathrm{NO}_{2}$, pois, segundo a regra de El-Sayed, a troca do spin deve ser acompanhada de mudanças na simetria espacial dos orbitais. 


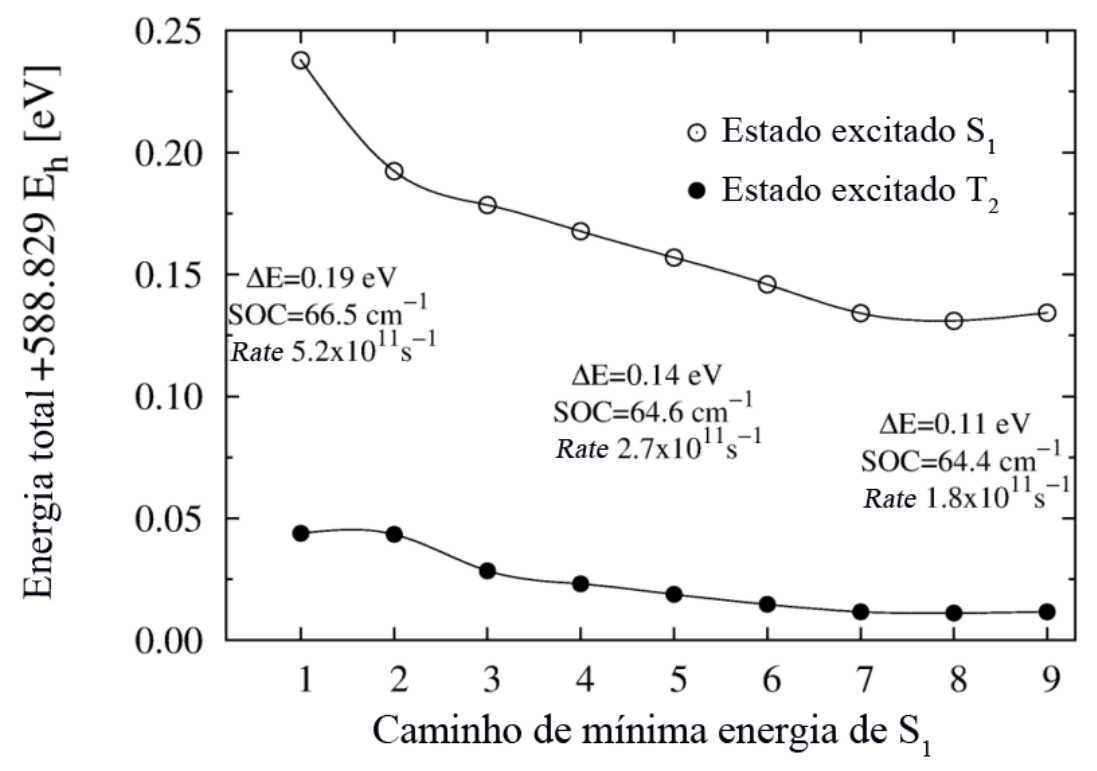

Figura 7.3. Perfil energético dos estados eletrônicos $S_{1}$ e $T_{2}$ calculados no MEP de $S_{1}$. Também são mostrados o SOC e o ISC rate calculados no primeiro, quinto e oitavo passo do MEP.

O ISC rate foi aproximadamente calculado usando a equação (7.2), sendo função da separação energética entre os estados eletrônicos $S_{1}$ e $T_{2}$. Estes estados eletrônicos são próximos ao longo do MEP de $\mathrm{S}_{1}$, como é indicado na figura 7.3, sendo este um elemento importante para obter um acoplamento vibracional significativo. A energia de reorganização $\lambda$ foi calculada no nível CASPT2, obtendo-se $-0.42 \mathrm{eV}$, ou seja, depois que o estado tripleto $T_{2}$ é populado ele alcança sua geometria de equilíbrio, localizada $0.42 \mathrm{eV}$ abaixo da energia do mínimo de $\mathrm{S}_{1}$. Os valores obtidos de ISC rate, mostrados na figura 7.3 , estão na faixa de $1.8 \times 10^{11}$ até $5.2 \times 10^{11} \mathrm{~s}^{-1}$. Estes valores indicam uma grande eficiência do ISC, mostrando que pode competir eficientemente com o decaimento por fluorescência do estado $S_{1}$, ainda mais quando a força de oscilador de $S_{1}$ para $\mathrm{S}_{0}$ na geometria de equilíbrio de $\mathrm{S}_{1}$ é pequena (0.0023). Na literatura aparecem valores reportados de ISC em sistemas moleculares que vão desde $10^{6}$ até $10^{11} \mathrm{~s}^{-1}[213]$. Embora o ISC rate tem valores altos ao longo do MEP, os valores máximos são atingidos nos primeiros passos da relaxação. Segundo a equação (7.3) o fator do acoplamento vibracional atinge valores máximos quando $|\Delta E| \approx \lambda$.

No sistema molecular nitrobenzeno também foi indicado por Takezaki et al. ${ }^{[214]}$ um intersystem crossing no decaimento não radiativo e resultados semelhantes foram obtidos por Quenneville et al. ${ }^{[215]}$ para o nitrobenzeno e outros compostos nitroaromáticos, embora apareçam algumas diferenças em relação aos nossos resultados 
para o 1-nitronaftaleno. Estes resultados parecem mostrar que o ISC é uma característica da fotofísica dos compostos nitroaromáticos, onde o decaimento não radiativo é principalmente influenciado pelas propriedades do grupo $\mathrm{NO}_{2}$. Os anéis aromáticos parecem ter uma importância menor nestes mecanismos de decaimento, ainda mais quando as mudanças geométricas no MEP do estado $\mathrm{S}_{1}$ e nas geometrias de equilíbrio dos estados excitados são principalmente caracterizadas por mudanças no grupo $\mathrm{NO}_{2}$, como se pode ver na figura 7.4

Embora os resultados mostrados para o 1-nitronaftaleno tenham sido obtidos na fase gasosa, eles podem ser considerados propriedades do sistema que podem ser levemente perturbadas pelo solvente. Isso é mostrado experimentalmente por Peon et al. ${ }^{[184]}$ onde o tempo de vida do estado $S_{1}$ continua sendo menor que 100 fs na presença de vários tipos de solventes.

$\mathrm{Na}$ figura 7.5 é mostrado o perfil energético dos estados eletrônicos que participam no mecanismo de desativação não radiativo proposto. A área sombreada representa a região mais favorável para o ISC. Esse modelo deve ser capaz de descrever as bandas de absorção transitórias obtidas experimentalmente por Zugazagoitia et. al ${ }^{[187]} \mathrm{e}$ Reichardt et al. ${ }^{[188]}$. Sendo esse é o objetivo da próxima seção.

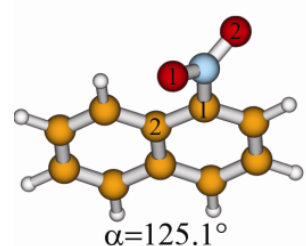

$\mathrm{S}_{0} \varphi=178.7^{\circ}$ $\tau=43.0^{\circ}$

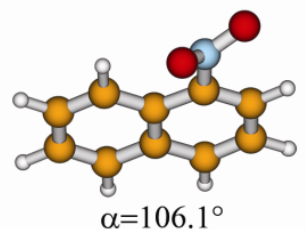

$\mathrm{S}_{1} \varphi=150.1^{\circ}$ $\tau=53.6^{\circ}$

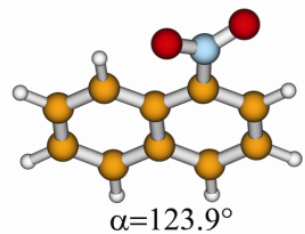

$\mathrm{T}_{1} \quad \varphi=179.0^{\circ}$ $\tau=24.0^{\circ}$

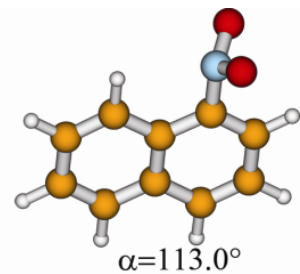

$\mathrm{T}_{2} \quad \varphi=137.6^{\circ}$ $\tau=111.4^{\circ}$

Figura 7.4. Estruturas otimizadas dos correspondentes estados eletrônicos. Os ângulos que definem o grupo $\mathrm{NO}_{2}$ são: $\alpha$ = ângulo $\left(\mathrm{O}_{1} \mathrm{NO}_{2}\right), \varphi=$ diedro $\left(\mathrm{O}_{1} \mathrm{O}_{2} \mathrm{NC}_{1}\right)$ e $\tau=$ diedro $\left(\mathrm{O}_{1} \mathrm{NC}_{1} \mathrm{C}_{2}\right)$.

Na figura 7.4 são mostradas as estruturas otimizadas para cada estado eletrônico e são indicados os ângulos que definem o grupo $\mathrm{NO}_{2}$. A geometria de equilíbrio do estado fundamental $\left(\mathrm{S}_{0}\right)$ apresenta o grupo $\mathrm{NO}_{2}$ rotacionado num ângulo de $43^{\circ}$ em relação ao plano dos anéis aromáticos e não forma estrutura piramidal. Quando o sistema relaxa, após a transição de absorção, para a geometria de equilíbrio do estado eletrônico $\mathrm{S}_{1} \mathrm{o}$ grupo $\mathrm{NO}_{2}$ adota uma geometria piramidal. Uma vez que o estado eletrônico $\mathrm{T}_{2}$ é populado, ele atinge a geometria de equilíbrio com o grupo $\mathrm{NO}_{2}$ bastante mais 
rotacionado em relação ao plano dos anéis e formando uma estrutura ligeiramente piramidal. Na geometria de equilíbrio do estado $\mathrm{T}_{1}$ o plano do grupo $\mathrm{NO}_{2}$ forma $23^{\circ}$ com o plano dos anéis e não tem ângulo piramidal.

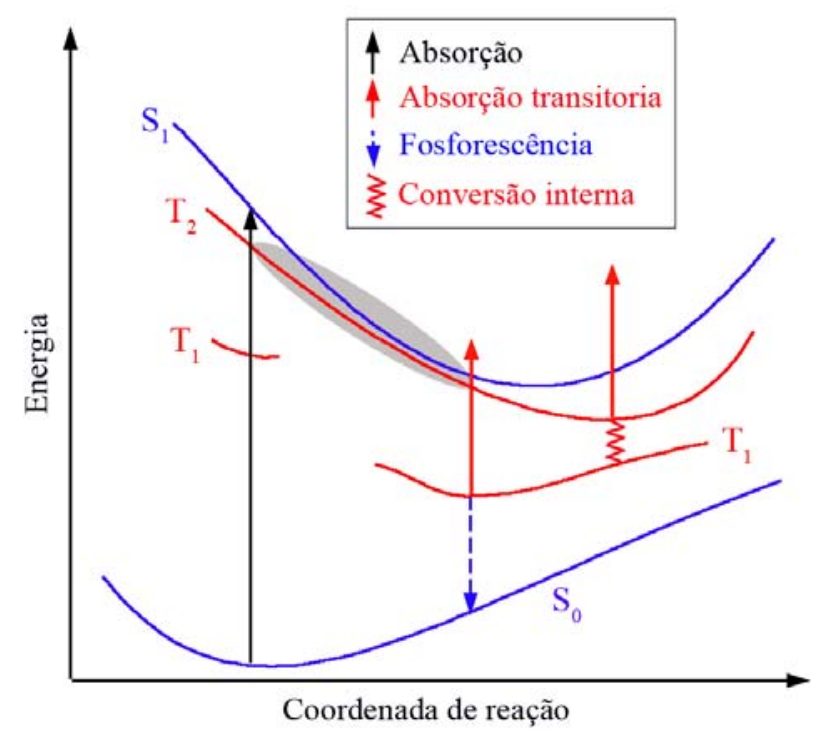

Figura 7.5. Perfil energético esquemático dos estados eletrônicos envolvidos na fotofísica do 1nitronaftaleno e transições eletrônicas discutidas no trabalho. A área sombreada é favorável para o ISC.

\subsubsection{Espectros de absorção transitória incluindo o efeito de solventes}

A dinâmica dos estados eletrônicos excitados do sistema molecular 1-nitronaftaleno foi estudada por Zugazagoitia et. al ${ }^{[187]}$ e Reichardt et al. ${ }^{[188]}$ usando espectroscopia de absorção transitória. Nestes experimentos foi observado que na medida em que desaparece a emissão do estado $\mathrm{S}_{1}$, um estado tripleto intermediário começa a ser populado. Este estado intermediário é detectado por uma banda de absorção transitória, com máximo entre 350 e $400 \mathrm{~nm}$, que é observada ao redor de 200 fs após a transição de absorção do estado fundamental. Esse processo é explicado aqui através do eficiente ISC entre $S_{1}$ e $T_{2}$ discutido anteriormente. Posteriormente, passado entre 2 e 45 ps duas novas bandas de absorção começam a aparecer; uma banda larga na região visível entre 500 e $600 \mathrm{~nm}$ e outra na região UV com máximo ao redor de $400 \mathrm{~nm}$. Estas bandas de absorção são atribuídas ao estado eletrônico $T_{1}$, que é populado através da conversão interna com o estado intermediário. A evolução espectral do estado eletrônico $T_{1}$ 
depende do esfriamento vibracional, que alcança a estabilidade em poucas dezenas de picosegundos ${ }^{[187,188]}$.

Estas bandas de absorção transitória devem ser descritas corretamente a fim de avaliar o modelo de desativação proposto (figura 7.5). O estudo das transições eletrônicas levara em consideração o efeito de solvente a fim de poder comparar corretamente com os resultados experimentais. Esse é o objetivo desta seção, onde os espectros de absorção, absorções transitórias e fosforescência são estudados em fase gasosa e em solvente.

Para descrever o efeito do solvente na transição de absorção, foi feita uma simulação do solvente metanol em equilíbrio eletrostático com o estado fundamental do 1-nitronaftaleno. Outra simulação foi realizada usando metanol em desequilíbrio eletrostático com o segundo estado tripleto, a fim de descrever o espectro de absorção transitória observada ao redor dos 200 fs, que corresponderia no experimento ${ }^{[188]}$ à absorção do estado tripleto intermediário. Também foram feitas duas simulações usando metanol e etanol em equilíbrio eletrostático com o primeiro estado tripleto, com o objetivo de descrever as bandas de absorção transitória observadas entre os 2 e 45 ps [187, 188] e o espectro de fosforescência ${ }^{[185]}$. As geometrias usadas na descrição das transições eletrônicas são as configurações de equilíbrio do estado eletrônico onde a transição se origina.

Antes de analisar o efeito de solvente nas transições eletrônicas, é conveniente discutir a polarização eletrônica do soluto nos diferentes estados eletrônicos. Na figura 7.6 são mostradas as variações dos momentos de dipolo médio obtidas nos processos iterativos QM/MM de polarização eletrônica. Como se pode ver na figura 7.6a, o estado $\mathrm{T}_{2}$ apresenta uma intensa polarização eletrônica quando interage com o solvente metanol. O momento de dipolo aumenta notavelmente desde 4.5 D na fase gasosa até 21.86 D em solvente. Segundo nossos resultados, este estado eletrônico é o estado intermediário entre o estado $S_{1}$ e os estados tripletos no mecanismo de decaimento não radiativo. No estado eletrônico fundamental do 1-nitronaftaleno o momento de dipolo aumenta ao redor de $36 \%$ comparado com a fase gasosa, alcançando $6.85 \mathrm{D}$ em metanol. $\mathrm{O}$ primeiro estado tripleto $\mathrm{T}_{1}$ é também polarizado aumentando o momento de dipolo em relação à fase gasosa ao redor de 64\% em metanol e 49\% em etanol (figura $7.6 b)$. 

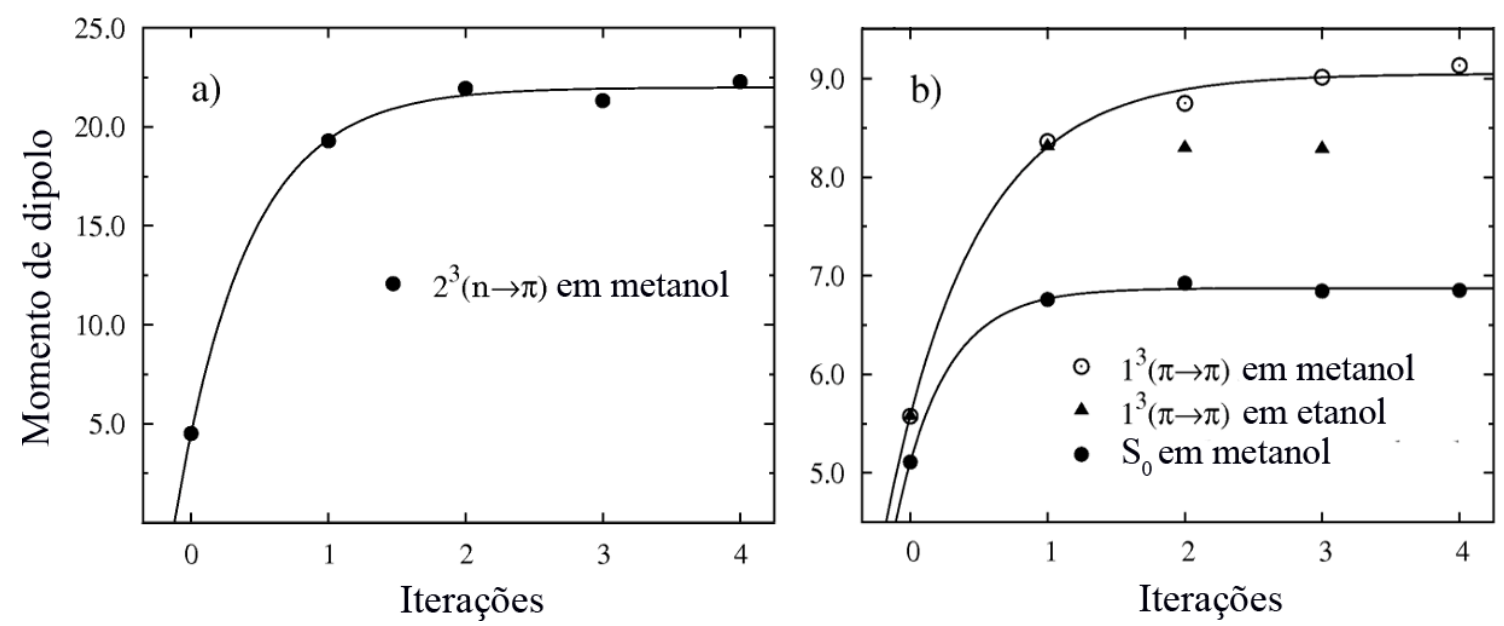

Figura 7.6. Variações dos momentos de dipolo dos estados eletrônicos em relação ao número de iterações no procedimento iterativo de polarização eletrônica.

Consideremos agora as transições eletrônicas começando a discussão com a transição de absorção para o estado eletrônico $S_{1}$ e o espectro de fosforescência. Esses dois processos são mostrados na figura 7.5 pelas setas preta e azul respectivamente. $\mathrm{Na}$ tabela 7.1 são mostrados resultados teóricos e experimentais já publicados na literatura a serem comparados com nossos resultados. Também são mostrados resultados teóricos e experimentais do decaimento fosforescente. O resultado teórico da transição de absorção, obtido neste trabalho no nível CIS(D)/aug-cc.pVDZ, indica uma transição de intensidade média com uma força de oscilador de 0.13 , em boa concordância com outros resultados anteriores que reportam 0.10 e 0.18 em fase gasosa ${ }^{[184,185]}$. A energia de transição para $\mathrm{S}_{1}$, calculada em fase gasosa, é de $4.11 \mathrm{eV}$, concordando muito bem com o resultado de Mikula et al. ${ }^{[185]}$ de $4.12 \mathrm{eV}$ usando o método semiempírico CNDO-CI, enquanto que usando DFT (TD-PBE0) Zugazagoitia et. al obtiveram 3.62 $\mathrm{eV}$.

$\mathrm{O}$ resultado obtido das simulações de $\mathrm{MC}$ considerando o efeito do solvente metanol na transição de absorção para $S_{1}$ indica um deslocamento para o vermelho de $0.18 \mathrm{eV}$, mudando a energia de absorção para $3.93 \mathrm{eV}$. Esse resultado concorda muito bem com os resultados experimentais ${ }^{[184,185]}$, onde se obteve um máximo da banda ao redor dos $3.81 \mathrm{eV}$, com uma largura da banda na metade da intensidade (FWHM) de $0.78 \mathrm{eV}$ (tabela 7.1). $\mathrm{O}$ espectro de fosforescência correspondente ao estado tripleto $\mathrm{T}_{1}$ apresenta um pequeno deslocamento para o vermelho de $0.03 \mathrm{eV}$, mudando de $2.70 \mathrm{eV}$ em fase gasosa para $2.67 \mathrm{em}$ etanol. Este resultado concorda razoavelmente bem com o máximo experimental de $2.38 \mathrm{eV}^{[185]}$. 
Tabela 7.1. Espectros de absorção e fosforescência do 1-nitronaftaleno. Entre parêntesis aparece o intervalo energético que define a largura da banda na metade da intensidade.

\begin{tabular}{|c|c|c|c|c|}
\hline & \multicolumn{2}{|c|}{ Absorção (eV) } & \multicolumn{2}{|c|}{ Fosforescência (eV) } \\
\hline & gás & solvente/metanol & gás & solvente/etanol \\
\hline $\begin{array}{l}\text { Este trabalho } \\
\text { CIS(D)/AVDZ }\end{array}$ & 4.11 & $\begin{array}{c}3.93 \\
\text { simulação }\end{array}$ & 2.70 & $\begin{array}{c}2.67 \\
\text { simulação }\end{array}$ \\
\hline Mikula et al. ${ }^{[185]}$ & $\begin{array}{c}4.12 \\
\text { CNDO-CI }\end{array}$ & $\begin{array}{c}3.81(3.26-4.67)^{\mathrm{a}} \\
\text { exp. }\end{array}$ & - & $\begin{array}{l}2.38 \\
\text { exp. }\end{array}$ \\
\hline $\begin{array}{l}\text { Zugazagoitia } \\
\text { et al. }\end{array}$ & $\begin{array}{c}3.62 \\
\text { DFT/PBE0 }\end{array}$ & $\begin{array}{l}3.81(3.35-4.13) \\
\text { exp. }\end{array}$ & - & - \\
\hline $\begin{array}{l}\text { Reichardt } \\
\text { et al. }{ }^{[188]}\end{array}$ & & $\begin{array}{c}3.80(3.40-4.18) \\
\exp \end{array}$ & - & - \\
\hline
\end{tabular}

${ }^{a}$ Este resultado experimental obteve-se usando o solvente isopentano.

O espectro de absorção transitória, observado ao redor dos 200 fs após a transição para o estado $\mathrm{S}_{1}$, foi obtido experimentalmente por Reichard et al. ${ }^{[188]}$ e foi relacionado com a absorção do estado tripleto receptor. Portanto, a descrição teórica deste espectro é de muita importância no entendimento da fotofísica do 1-nitronaftaleno, pois o tripleto receptor é o intermediário entre o estado eletrônico $S_{1}$ e os estados tripletos na desativação não radiativa. Conforme o mecanismo de desativação proposto neste trabalho esse espectro deve corresponder a transições de absorção do estado eletrônico $\mathrm{T}_{2}$. Nesse sentido, usando a geometria de equilíbrio do estado eletrônico $\mathrm{T}_{2}$ (figura 7.4) obtiveram-se em fase gasosa duas transições eletrônicas, $\mathrm{T}_{2} \rightarrow \mathrm{T}_{6}$ e $\mathrm{T}_{2} \rightarrow \mathrm{T}_{7}$, com energias de 3.39 e 3.64 eV respectivamente, calculadas no nível CIS(D)/aug-cc-pVDZ. Estas transições poderiam ser associadas à banda experimental ${ }^{[188]}$ obtida em solução de metanol com máximo em $3.35 \mathrm{eV}$. Estes resultados são mostrados na tabela 7.2. Como já foi discutido anteriormente, a polarização eletrônica do 1-nitronaftaleno no estado eletrônico $\mathrm{T}_{2}$ é muito grande, aumentando consideravelmente o momento de dipolo em metanol (figura 7.6a). Porém, levando em consideração que os resultados experimentais de Zugazagoitia et al. ${ }^{[187]}$ e Reichardt et al. ${ }^{[188]}$ indicam que a conversão interna entre o estado intermediário e o estado fosforescente $T_{1}$ ocorre em escalas de tempo de entre $1 \mathrm{a}$ 16 ps e 2 a 4 ps respectivamente, pode-se inferir que o 1-nitronaftaleno no estado eletrônico $T_{2}$ não tem tempo suficiente para atingir o equilíbrio eletrostático solutosolvente mostrado na figura 7.6a. Baseados nisto, as transições de absorção $T_{2} \rightarrow T_{6} e$ $\mathrm{T}_{2} \rightarrow \mathrm{T}_{7}$ em metanol devem ser calculadas usando configurações soluto-solvente que carreguem informação do solvente em equilíbrio eletrostático com o estado eletrônico anterior, ou seja, o estado fundamental. Ao mesmo tempo a resposta rápida do solvente 
deve ser equilibrada com a nova distribuição eletrônica do 1-nitronaftaleno no estado $\mathrm{T}_{2}$. Este comportamento físico do sistema foi levado em consideração de forma aproximada neste trabalho realizando uma simulação de MC com o 1-nitronaftaleno no estado eletrônico $T_{2}$, mas, começando a simulação desde configurações soluto-solvente anteriormente equilibrada com o estado fundamental. $\mathrm{O}$ momento de dipolo calculado em solvente depois da simulação de MC foi de 5.23 D, mostrando, em concordância com a figura 7.6a, que o sistema no estado eletrônico $\mathrm{T}_{2}$ está longe do equilíbrio eletrostático soluto-solvente. Usando as configurações soluto-solvente obtidas dessa simulação, foi calculado o deslocamento espectral das transições $T_{2} \rightarrow T_{6}$ e $T_{2} \rightarrow T_{7}$, anteriormente calculas em fase gasosa. Os valores médios das energias de transição obtidos foram 3.37 e $3.45 \mathrm{eV}$ para $\mathrm{T}_{2} \rightarrow \mathrm{T}_{6}$ e $\mathrm{T}_{2} \rightarrow \mathrm{T}_{7}$ respectivamente. Esses valores estão em excelente concordância com a banda experimental com máximo em $3.35 \mathrm{eV}$, obtida por Reichardt ${ }^{[188]}$ com FWHM de 0.34 eV (tabela 7.2).

Tabela 7.2. Espectros de absorções transitórias. Entre parêntesis aparece o intervalo energético que define a largura da banda na metade da intensidade.

\begin{tabular}{|c|c|c|c|c|c|c|}
\hline & \multirow{2}{*}{\multicolumn{2}{|c|}{ Abs. trans. sub-200 fs (eV) }} & \multicolumn{4}{|c|}{ Abs. Trans. 2-45 ps (eV) } \\
\hline & & & \multicolumn{2}{|c|}{$1^{\mathrm{a}}$ banda } & \multicolumn{2}{|c|}{$2^{\mathrm{a}}$ banda } \\
\hline & $\begin{array}{c}\text { gás } \\
\mathrm{T}_{2} \rightarrow \mathrm{T}_{6} / \mathrm{T}_{2} \rightarrow \mathrm{T}_{7}\end{array}$ & $\begin{array}{c}\text { solvente metanol } \\
\mathrm{T}_{2} \rightarrow \mathrm{T}_{6} / \mathrm{T}_{2} \rightarrow \mathrm{T}_{7}\end{array}$ & gás & $\begin{array}{c}\text { solvente } \\
\text { metanol/etanol }\end{array}$ & gás & $\begin{array}{c}\text { solvente } \\
\text { metanol/etanol }\end{array}$ \\
\hline $\begin{array}{c}\text { Este trabalho } \\
\text { CIS(D)/AVDZ }\end{array}$ & $3.39 / 3.64$ & $\begin{array}{l}3.37 / 3.45 \\
\text { simulação }\end{array}$ & 2.40 & $\begin{array}{l}2.25 / 2.29 \\
\text { simulação }\end{array}$ & 3.33 & $\begin{array}{l}3.23 / 3.25 \\
\text { simulação }\end{array}$ \\
\hline $\begin{array}{l}\text { Zugazagoitia } \\
\text { et al. }{ }^{[187]} \text { (exp) }\end{array}$ & - & - & - & $\begin{array}{c}2.11 \\
(1.91-2.34)\end{array}$ & - & \\
\hline $\begin{array}{c}\text { Reichardt } \\
\text { et al. }{ }^{[188]}(\exp )\end{array}$ & - & $\begin{array}{c}3.35 \\
(3.20-3.54)\end{array}$ & - & $\begin{array}{c}2.11 \\
(1.91-2.36)\end{array}$ & - & $\begin{array}{c}3.01 \\
(2.83-3.31)\end{array}$ \\
\hline
\end{tabular}

Como foi comentado anteriormente, na medida em que desaparece a absorção transitória observada ao redor dos 200 fs o estado tripleto $T_{1}$ começa a ser populado através da CI desde o estado tripleto intermediário. Este estado eletrônico é detectado por duas bandas de absorção transitória que se observam entre 2 e 45 ps após a absorção do estado fundamental. Na tabela 7.2 são mostradas estas bandas experimentais de absorção transitória. No trabalho de Zugazagoitia et al. ${ }^{[187]}$ somente foi descrita uma banda de absorção devido à região espectral analisada, enquanto Reichardt et al. ${ }^{[188]}$ observaram as duas bandas de absorção transitória. As transições eletrônicas que descrevem estas bandas de absorção transitória, obtiveram-se no presente trabalho usando a geometria otimizada do estado tripleto $T_{1}$ (figura 7.4 ) e considerando o solvente em equilíbrio eletrostático com este estado eletrônico. A tabela 7.2 mostra as 
energias de absorção calculadas no nível CIS(D)/aug-cc-pVDZ na fase gasosa e em solução. A primeira banda é descrita pela transição eletrônica $T_{1} \rightarrow T_{3}$ de $2.40 \mathrm{eV} \mathrm{em}$ fase gasosa, que é deslocada para 2.25 e $2.29 \mathrm{eV}$ nos solventes metanol e etanol respectivamente. Estes resultados concordam muito bem com os resultados experimentais, ficando muito próximos do máximo da banda de $2.11 \mathrm{eV}$ com um FWHM de $0.44 \mathrm{eV}$. A segunda banda de absorção transitória é descrita pela transição $\mathrm{T}_{1} \rightarrow \mathrm{T}_{5}$ mudando de $3.33 \mathrm{eV}$ na fase gasosa para 3.23 e $3.25 \mathrm{eV}$ nos solventes metanol e etanol respectivamente. Embora estes valores estejam um pouco mais afastados do máximo da banda ainda continuam dentro do FWHM experimental.

Resumidamente, a banda de absorção transitória observada experimentalmente ao redor dos $200 \mathrm{fs}^{[188]}$ é descrita aqui pelas transições $\mathrm{T}_{2} \rightarrow \mathrm{T}_{6}$ e $\mathrm{T}_{2} \rightarrow \mathrm{T}_{7}$ que aparecem próximas uma da outra. Isto indica que o estado $T_{2}$ é o tripleto intermediário entre $S_{1} e$ os estados tripletos, como foi anteriormente indicado pelo eficiente ISC entre $\mathrm{S}_{1}$ e $\mathrm{T}_{2}$. As bandas de absorção transitória observadas entre 2 e 45 ps ${ }^{[187,188]}$ são descritas pelas transições $\mathrm{T}_{1} \rightarrow \mathrm{T}_{3}$ e $\mathrm{T}_{1} \rightarrow \mathrm{T}_{5}$, apoiando a idéia da CI entre o estado tripleto intermediário e $\mathrm{T}_{1}$.

\subsection{Conclusões}

A ultra-rápida troca de multiplicidade, observada experimentalmente após a transição de absorção para o estado $S_{1}$, foi basicamente explicada no presente trabalho pelo eficiente ISC entre os estado singletos e tripletos. Este mecanismo envolve um estado tripleto intermediário especifico $\left(\mathrm{T}_{2}\right)$ que apresenta um acoplamento spin-órbita consideravelmente grande com o estado singleto $\mathrm{S}_{1}$. Os valores consideravelmente altos do ISC rate são consequência dos grandes valores de SOC e do acoplamento vibracional aproximadamente calculado. Estes resultados junto a outros resultados teóricos e experimentais parecem mostrar uma característica comum dos compostos nitroaromáticos, onde a fotofísica destes sistemas é fortemente determinada pelo ISC entre os estados singletos e tripletos.

Usando o modelo de desativação não radiativo proposto, foram descritos teoricamente neste trabalho os espectros de absorções transitórias observados experimentalmente. Para isso foi considerando o efeito de solvente no estado fundamental e estados excitados, a fim de comparar corretamente com os experimentos. 
Os resultados teóricos apresentados indicam que o decaimento não radiativo do sistema molecular 1-nitronaftaleno é descrito da seguinte maneira: após a transição de absorção para o estado excitado $\mathrm{S}_{1}$, o sistema passa rapidamente, em escalas de tempo de $100 \mathrm{fs}$, para o estado tripleto $\mathrm{T}_{2}$ através de um eficiente ISC entre estes estados eletrônicos. Nas próximas dezenas de picosegundos acontece uma conversão interna entre os estados $T_{2}$ e $T_{1}$. Neste último estado eletrônico o sistema permanece e decai em escalas de tempo maiores ao estado fundamental, indicado pelo espectro de fosforescência deste estado. 
126 Estudo teórico da fotofísica do sistema molecular 1-nitronaftaleno 


\section{Capítulo 8}

\section{Efeito do solvente no Stokes shift e no decaimento não fluorescente do}

sistema molecular daidzein

Os flavonóides são uma família de compostos polifenólicos com propriedades antioxidantes diretamente ligadas a muitos benefícios para a saúde ${ }^{[216-219]}$. Segundo o paradoxo francês, embora os franceses tenham estilos de vida menos saudáveis que os americanos, os franceses têm menores índices de doenças cardíacas que os americanos ${ }^{[220,221]}$. Vários estudos ${ }^{[222-226]}$ têm indicado que o consumo frequente de vinho tinto é a explicação do paradoxo, pois, os flavonóides são abundantes na semente e na pele da uva e, portanto, no vinho tinto.

Por essas e outras razões, os flavonóides têm sido muito estudado. Em particular, as propriedades de fluorescência destes compostos tem sido objetivo de muitos trabalhos experimentais ${ }^{[227-240]}$, incrementando-se assim, o interesse no estudo teórico de estados excitados destes compostos ${ }^{[241-243]}$. Na literatura são reportadas varias características da fluorescência dos flavonóides. Nos sistemas moleculares 3-hidroxiflavones (flavonoles) têm sido observados grandes valores de Stokes shift em solução. A fluorescência, observada ao redor de $500 \mathrm{~nm}$ é originada num estado excitado que é caracterizado pela transferência intramolecular de um próton, do grupo 3-hidróxila para o grupo 4carbonila ${ }^{[227,231,233]}$. Outros sistemas caracterizados pela transferência intramolecular 
de próton são os 5-hidroxiflavones, nos quais são observadas duas bandas de emissão experimentais ${ }^{[238]}$. Por outro lado, nos 7-hidroxiflavones são também observados grandes valores de Stokes shift em álcool e em água, mas, nestes casos a fluorescência não é atribuída à transferência intramolecular de próton. Nestes sistemas, são normalmente observadas duas bandas de emissão em soluções com $\mathrm{pH}$ maior que 2, a mais intensa é associada à emissão da forma aniônica e a banda menos intensa é relacionada com o tautômero keto. Em soluções com pH menor que 2 a fluorescência é associada à forma zwitterionica do tautômero enol ${ }^{[230,239,240]}$.

Os isoflavones são um subgrupo de flavonóides, mostrados na figura 8.1, que também têm sido objeto de vários estudos, tanto experimentais quanto teóricos. Numa ampla variedade destes compostos têm sido obtidos grandes valores de Stokes shift, na ordem de $1.4 \mathrm{eV}$ em alguns tipos de solventes ${ }^{[228,229,236,243]}$. A fluorescência destes sistemas moleculares tem sido observada tanto em condições básicas como neutras ${ }^{[228,}$ 236, 237] e em alguns destes compostos não é observado dependência com o pH ${ }^{[236]}$. As evidências experimentais relacionadas com estes compostos (ver discussão na Ref. ${ }^{[243]}$ ) indicam que a fluorescência pode ser atribuída à forma neutra.

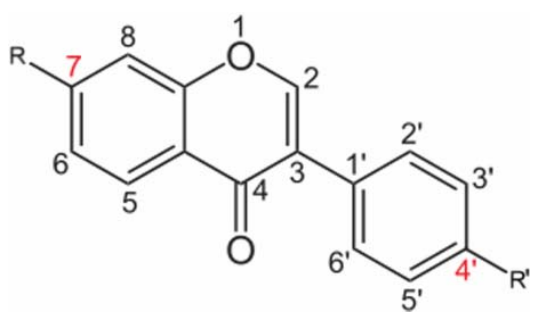

Figura 8.1. Representação do subgrupo dos isoflavones. O daidzein é formado por $\mathrm{R}=\mathrm{OH}$ e $\mathrm{R}^{\prime}=\mathrm{OH}$.

No presente trabalho, estamos particularmente interessados no sistema molecular daidzein como sistema modelo. Este sistema pertence ao grupo dos isoflavones, como indicado na figura 8.1, com $\mathrm{R}=\mathrm{OH}$ e $\mathrm{R}^{\prime}=\mathrm{OH}$. Nosso interesse no estudo deste composto surge da necessidade de explicar os grandes valores de Stokes shift observados experimentalmente no subgrupo dos isoflavones, pois a fluorescência não é originada em estados excitados deprotonados e a transferência intramolecular de próton não é possível devido a grande separação entre os grupos hidroxila e carbonila.

O sistema molecular daidzein mostra valores consideravelmente grandes de Stokes shift em solventes polares próticos como a água e o metanol, mas curiosamente, em 
solvente polar aprótico como a acetonitrila não é observada fluorescência ${ }^{[237,243]}$. Portanto o objetivo do presente trabalho é esclarecer teoricamente as causas dos grandes valores de Stokes shift em solventes polares próticos como a água e também estudar o mecanismo de decaimento não fluorescente em acetonitrila. Na literatura foi publicado recentemente um estudo teórico ${ }^{[243]}$ que tenta explicar o papel do solvente no Stokes shift do daidzein usando um modelo de microsolvatação, mas, neste modelo não é descrito corretamente o Stokes shift nem o papel do solvente.

\subsection{Detalhes computacionais}

As energias dos estados eletrônicos excitados do daidzein foram calculadas usando a teoria perturbativa multiconfiguracional de segunda ordem (CASPT2) ${ }^{[30]}$ usando a função de onda referência CASSCF. No cálculo CASSCF, 12 elétrons foram correlacionados em 12 orbitais, onde os orbitais ativos são os cinco orbitais $\pi$ e seis $\pi^{*}$ que melhor descrevem os três estados excitados $\pi \pi^{*}$ de menos energias. Também foi adicionado o orbital lone-pair do oxigênio a fim de descrever o estado eletrônico excitado $n \pi *$. Esses orbitais ativos foram selecionados da mesma forma que no trabalho do capítulo anterior. Foi usado o conjunto de funções-base ANO-L, contraídas a C, O $[4 \mathrm{~s} 3 \mathrm{p} 1 \mathrm{~d}] \mathrm{c} / \mathrm{H}[2 \mathrm{~s} 1 \mathrm{p}]^{[194,195]}$ que considera funções difusas e polarizadas nos átomos não hidrogênicos. Os elétrons do caroço 1s dos átomos de oxigênio e carbono novamente foram mantidos congelados na perturbação de segunda ordem. Os cálculos CASSCF, na determinação dos espectros de absorção e de emissão, são realizados usando o procedimento state average considerando os cinco estados eletrônicos de menores energias.

No estudo das transições de absorção do daidzein foi usada a geometria de equilíbrio do estado eletrônico fundamental, otimizada em fase gasosa no nível MP2 com o conjunto de funções-base $6-31+\mathrm{G}(\mathrm{d})$. A otimização foi calculada usando o pacote de programas Gaussian $03^{[164]}$.

O estudo do espectro de fluorescência foi orientado para a identificação do estado eletrônico mais populado nas transições de absorção e acompanhar sua evolução em solvente. As otimizações de geometria dos estados eletrônicos excitados de interesse foram calculadas no nível CASSCF/ANO-L em fase gasosa, considerando o mesmo 
espaço ativo discutido anteriormente. Todos os cálculos multiconfiguracionais foram realizados usando o pacote de programas MOLCAS $7.4^{[201]}$.

As otimizações das geometrias de equilíbrio do estado eletrônico fundamental e excitados foram calculadas em fase gasosa, pois, achamos que na presença de solvente não devem aparecer mudanças geométricas tão significativas.

Os solventes considerados neste trabalho foram água e acetonitrila, como foi usado nos trabalhos experimentais. $\mathrm{O}$ efeito de solvente foi levado em consideração usando a metodologia sequencial QM/MM ${ }^{[160]}$ mostrada no capítulo 3 e usada nos trabalhos apresentados anteriormente nesta tese, onde as simulações de MC são usadas para gerar configurações soluto-solvente a serem usadas nos cálculos de mecânica quântica. Assim, como no trabalho mostrado no capítulo anterior, o efeito de solvente é levado em consideração no estudo dos estados eletrônicos excitados. As simulações foram feitas no ensemble NPT, considerando uma molécula de daidzein e 700 moléculas de solvente em condições normais de temperatura e pressão. Os parâmetros de LennardJones para o daidzein na interação intermolecular foram obtidos dos trabalhos de Jorgensen et al. ${ }^{[244]}$. Para as moléculas de água foi usado o modelo TIP3P [161], enquanto para a acetonitrila foi usado o modelo proposto por Böhm e McDonald ${ }^{\text {[245] }}$. As cargas atômicas $q_{i}$ foram calculadas considerando a polarização eletrônica de cada estado eletrônico estudado. Para isso foi novamente usado o procedimento iterativo mostrado nos trabalhos anteriores e na seção (3.4). As cargas dos sítios atômicos foram calculadas usando o método ESPF ${ }^{[246]}$ (ElectroStatic Potential Fitted), implementado no pacote de programas MOLCAS $7.4^{[201]}$.

Em cada simulação de Monte Carlo são geradas $3.0 \times 10^{5}$ configurações solutosolvente, de onde são selecionadas 100 configurações estatisticamente descorrelacionadas para estudar as propriedades quântica. Novamente, os cálculos de absorção e emissão do daidzein em solvente são feitos usando o modelo ASEC ${ }^{[80]}$, pois considerar as moléculas de solvente explicitamente envolve um custo computacional muito alto neste sistema.

A fim de explicar o decaimento não fluorescente do daidzein em acetonitrila, considerou-se estudar o decaimento através do intersystem crossing. Nesse sentido, foram calculados elementos de acoplamento spin-órbita entre estados singletos e tripletos usando a aproximação do hamiltoniano efetivo de um elétron ${ }^{[34]}$ na interação spin-órbita. Estes cálculos foram realizados da mesma forma que no trabalho do capítulo anterior, usando o programa RASSI ${ }^{[197]}$ implementado no MOLCAS $7.4^{[201]}$. 


\subsection{Resultados e discussão}

\subsubsection{Espectro de absorção}

Os espectros de absorção do daidzein, em água e em acetonitrila, foram determinados usando a geometria de equilíbrio do estado eletrônico fundamental, que é mostrada ilustrativamente na figura 8.2a. O ângulo diedro formado entre o chromone e o grupo fenil obtido na otimização é de $43.2^{\circ}$, concordando bastante bem com o resultado de Beyhan et al. ${ }^{[243]}$ de $39.9^{\circ}$, calculado no nível CC2/aug-cc-pVDZ.

a)

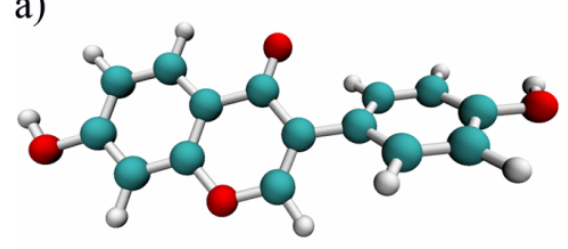

b)

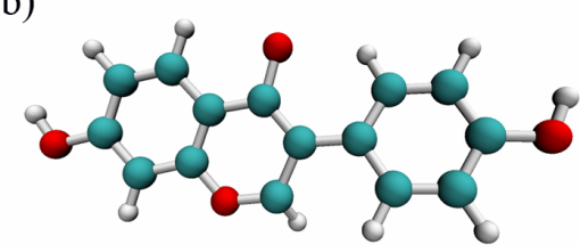

${ }^{1}\left(\pi \pi^{*} \mathrm{~L}_{\mathrm{b}}\right)$

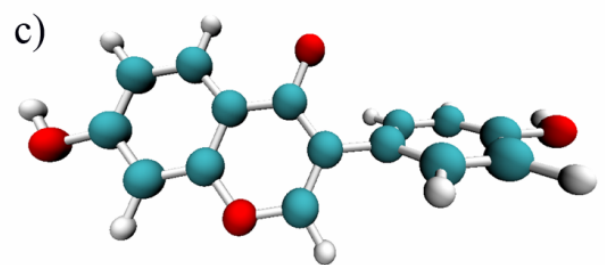

${ }^{1}\left(n \pi^{*}\right)$

Figura 8.2. Estruturas otimizadas dos estados eletrônicos indicados.

Para levar em consideração o efeito de solvente no espectro de absorção foi realizado o procedimento iterativo de polarização eletrônica já discutido, a fim de atingir o equilíbrio eletrostático soluto-solvente do daidzein no estado eletrônico fundamental. Nas figuras $8.3 \mathrm{a}$ e $8.3 \mathrm{~b}$ são mostradas as convergências dos momentos de dipolo obtidas em solvente, aumentando de $2.5 \mathrm{D}$ em fase gasosa até $10.0 \mathrm{D}$ em água e até $3.8 \mathrm{D}$ em acetonitrila. 

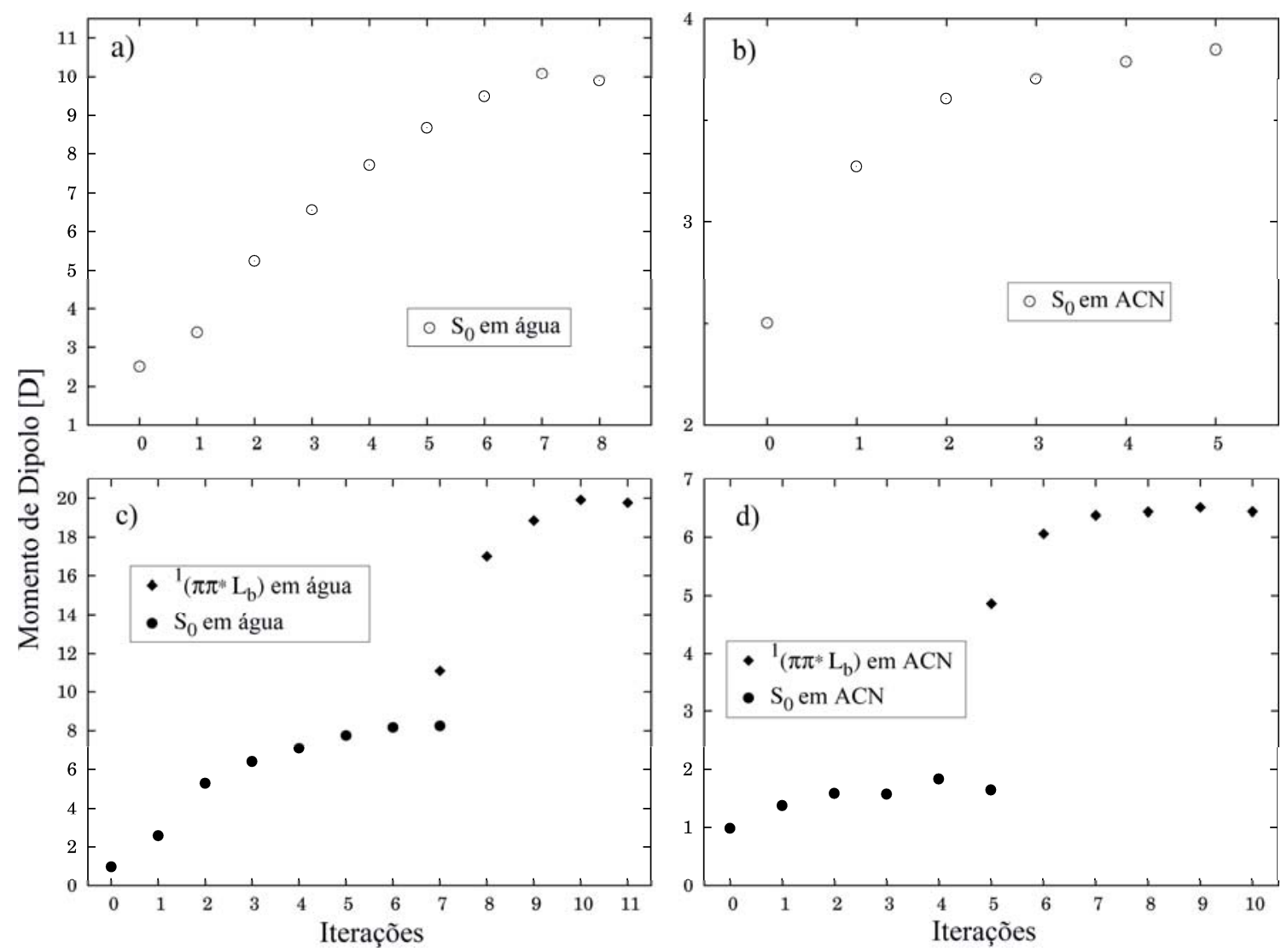

Figura 8.3. Mudanças dos momentos de dipolo calculados, nos correspondentes estados eletrônicos, em relação ao número de iterações no procedimento sequencial QM/MM de polarização eletrônica. ACN: acetonitrila.

$\mathrm{Na}$ tabela 8.1 são mostrados os quatro estados eletrônicos excitados menos energéticos calculados em solvente no nível CASPT2/ANO-L. Para diferenciar entre os estados eletrônicos $\pi \pi^{*}$ foi adotada a nomenclatura de Platt ${ }^{[247]}$, onde o estado ${ }^{1}\left(\pi \pi^{*} L_{a}\right)$ é caracterizado principalmente pela transição $\mathrm{HOMO} \rightarrow \mathrm{LUMO}$ e o estado ${ }^{1}\left(\pi \pi^{*} \mathrm{~L}_{\mathrm{b}}\right)$ por HOMO- $1 \rightarrow$ LUMO. O estado eletrônico ${ }^{1}\left(\pi \pi^{*}\right)$ é um estado principalmente caracterizado pela transição do HOMO-2 para o LUMO, enquanto o estado eletrônico ${ }^{1}\left(n \pi^{*}\right)$ se caracteriza principalmente pela transição desde o orbital lone-pair do oxigênio para o LUMO. Os orbitais moleculares que caracterizam estes estados eletrônicos são mostrados na figura 8.4 . 
Tabela 8.1. Energias de absorção para os quatro estados eletrônicos excitados de menos energia em água e acetonitrila e energia de emissão em água. Os valores energéticos estão indicados em $\mathrm{eV}$.

\begin{tabular}{|c|c|c|c|c|c|c|c|}
\hline \multicolumn{4}{|c|}{ água } & \multicolumn{4}{|c|}{ Acetonitrila } \\
\hline \multicolumn{8}{|c|}{ Absorção } \\
\hline Estado & $E_{\text {abs }}$ & $f$ & $\begin{array}{c}E_{\text {abs }}{ }^{\mathrm{a}} \\
\text { Exp. }^{[243]}\end{array}$ & Estado & $E_{\mathrm{abs}}$ & $f$ & $\begin{array}{c}E_{\text {abs }}{ }^{\mathrm{a}} \\
\text { Exp. }{ }^{243}\end{array}$ \\
\hline${ }^{1}\left(n \pi^{*}\right)$ & 4.14 & 0.070 & - & ${ }^{1}\left(n \pi^{*}\right)$ & 3.72 & 0.000 & - \\
\hline${ }^{1}\left(\pi \pi^{*} \mathrm{~L}_{\mathrm{b}}\right)$ & 4.16 & 0.196 & 4.09 & ${ }^{1}\left(\pi \pi^{*} \mathrm{~L}_{\mathrm{a}}\right)$ & 4.37 & 0.077 & 4.09 \\
\hline${ }^{1}\left(\pi \pi^{*} \mathrm{~L}_{\mathrm{a}}\right)$ & 4.70 & 0.165 & 5.00 & ${ }^{1}\left(\pi \pi^{*} \mathrm{~L}_{\mathrm{b}}\right)$ & 5.09 & 0.305 & 4.77 \\
\hline${ }^{1}\left(\pi \pi^{*}\right)$ & 5.44 & 0.057 & 5.85 & ${ }^{1}\left(\pi \pi^{*}\right)$ & 5.76 & 0.027 & 5.85 \\
\hline \multicolumn{8}{|c|}{ Emissão em água } \\
\hline \multicolumn{2}{|c|}{$\begin{array}{c}\text { Estado } \\
\text { fluorescente }\end{array}$} & \multicolumn{2}{|c|}{$E_{\mathrm{emi}}^{\mathrm{b}}$} & tokes shift $^{\mathrm{c}}$ & \multicolumn{2}{|c|}{$\begin{array}{c}E_{\text {emi }^{a}}{ }^{\mathrm{a}} \\
\text { Exp. }{ }^{243]}\end{array}$} & $\begin{array}{l}\text { Stokes shift } \\
\text { Exp. }{ }^{[243]}\end{array}$ \\
\hline \multicolumn{2}{|c|}{${ }^{1}\left(\pi \pi^{*} \mathrm{~L}_{\mathrm{b}}\right)$} & \multicolumn{2}{|c|}{$2.85 / 3.24$} & $1.31 / 0.92$ & \multicolumn{2}{|c|}{2.70} & 1.40 \\
\hline
\end{tabular}

${ }^{\mathrm{a}}$ Valores energéticos correspondentes aos máximos das bandas experimentais.

${ }^{\mathrm{b}}$ Energia de emissão calculada em equilíbrio/desequilíbrio eletrostático com o solvente.

${ }^{c}$ Stokes shift em relação à energia de absorção correspondente ao estado ${ }^{1}\left(\pi \pi^{*} \mathrm{~L}_{\mathrm{b}}\right)$. Essa é a energia experimental de excitação da fluorescência, em relação à qual é reportado o Stokes shift experimental.

Primeiramente mostraremos os resultados da absorção em água e posteriormente em acetonitrila. Segundo os resultados mostrados na tabela 8.1, o espectro de absorção calculado em água mostra duas transições intensas, com energias de $4.16 \mathrm{eV}(298 \mathrm{~nm}) \mathrm{e}$ $4.70 \mathrm{eV}(264 \mathrm{~nm})$, dadas pelas transições para ${ }^{1}\left(\pi \pi^{*} \mathrm{~L}_{\mathrm{b}}\right)$ e ${ }^{1}\left(\pi \pi^{*} \mathrm{~L}_{\mathrm{a}}\right)$ respectivamente. A primeira destas transições está em excelente concordância com a banda experimental observada com máximo em $4.09 \mathrm{eV}(303 \mathrm{~nm})$ e usada na excitação da fluorescência [243]. A segunda transições $(4.70 \mathrm{eV})$ está em muito boa concordância com a banda experimental observada com máximo em $5.00 \mathrm{eV}(248 \mathrm{~nm})$. Por outra parte, a quarta transição eletrônica mostrada, com energia de $5.44 \mathrm{eV}(228 \mathrm{~nm})$, concorda muito bem com a banda experimental que aparece na borda do espectro ao redor de $5.85(211 \mathrm{~nm})$. Finalmente, a transição para o estado eletrônico ${ }^{1}\left(n \pi^{*}\right)$, com energia de $4.14 \mathrm{eV} \mathrm{(300}$ $\mathrm{nm})$ não é observada experimentalmente pois está muito próxima de uma banda intensa.

Os resultados da absorção obtidos em acetonitrila são também mostrados na tabela 8.1. Como se pode ver, aparece só uma transição muito intensa, dada pela transição para o estado eletrônico ${ }^{1}\left(\pi \pi^{*} \mathrm{~L}_{\mathrm{b}}\right)$ com energia de $5.09 \mathrm{eV}(244 \mathrm{~nm})$. Esta transição está bastante próxima da banda principal, observada experimentalmente com máximo em $4.77 \mathrm{eV}(260 \mathrm{~nm})$. Outra transição, para o estado eletrônico ${ }^{1}\left(\pi \pi^{*} \mathrm{~L}_{\mathrm{a}}\right)$, menos intensa e 
com energia de $4.37 \mathrm{eV}(284 \mathrm{~nm})$, se encontra próxima de uma pequena banda observada em forma de ombro em $4.09 \mathrm{eV}(303 \mathrm{~nm}){ }^{[243]}$. Pode-se notar que estas duas transições eletrônicas encontram-se deslocadas simultaneamente para as regiões de maiores energias ao redor de $0.3 \mathrm{eV}$ (ou $15 \mathrm{~nm}$ ) em relação aos máximos das bandas experimentais. A transição de $5.76 \mathrm{eV}(215 \mathrm{~nm})$ para o estado eletrônico ${ }^{1}\left(\pi \pi^{*}\right)$ concorda muito bem com a banda observada na borda do espectro ao redor de $5.85 \mathrm{eV}$ $(212 \mathrm{~nm})$. Por outra parte o estado eletrônico ${ }^{1}\left(n \pi^{*}\right)$ parece ser um estado escuro, indicado pela força de oscilador igual a zero.

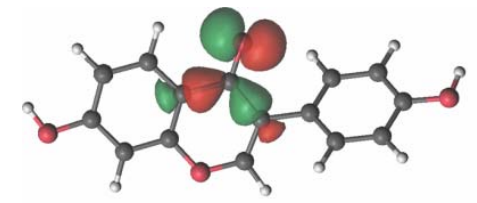

HOMO-5

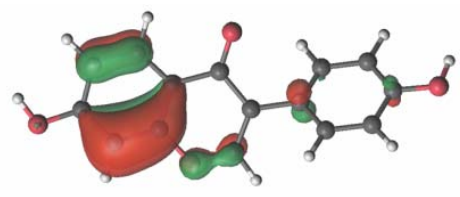

HOMO-2

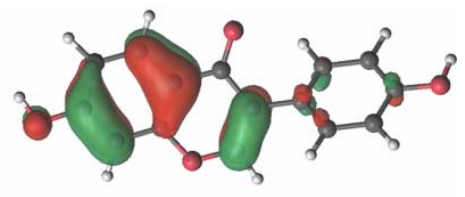

HOMO-1

(Lone-pair)

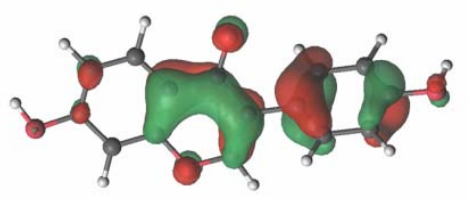

HOMO

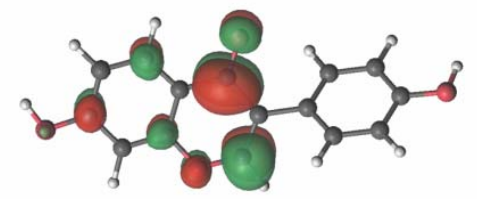

LUMO

Figura 8.4. Representação dos principais orbitais moleculares envolvidos nas transições eletrônicas desde o estado fundamental para os estado excitados.

\subsubsection{Espectro de fluorescência em água}

Baseado no espectro de absorção do daidzein, a descrição do espectro de emissão em água foi orientada ao estudo do estado eletrônico excitado ${ }^{1}\left(\pi \pi^{*} \mathrm{~L}_{\mathrm{b}}\right)$, desde que este estado é bastante populado nas transições de absorção (ver tabela 8.1) e reproduz fielmente a energia de excitação da fluorescência dos experimentos ${ }^{[243]}$. Nesse sentido, foi otimizado este estado eletrônico no nível CASSCF/ANO-L. Na estrutura otimizada, mostrada na figura $8.2 \mathrm{~b}$, o chromone e o grupo fenil formam aproximadamente uma configuração planar.

A solvatação do estado eletrônico ${ }^{1}\left(\pi \pi^{*} \mathrm{~L}_{\mathrm{b}}\right)$ em água foi estudada em duas etapas. Primeiramente, considerando que o tempo de vida deste estado eletrônico é curto e não é atingido o equilíbrio eletrostático soluto-solvente. Nesse caso são obtidas 
configurações soluto-solvente na geometria de equilíbrio de ${ }^{1}\left(\pi \pi^{*} \mathrm{~L}_{\mathrm{b}}\right)$ onde o solvente se encontra em equilíbrio eletrostático com o estado eletrônico fundamental. Esse procedimento é representado na figuras $8.3 \mathrm{c}$ pelos pontos circulares, onde a convergência destes pontos indica o equilíbrio com o estado eletrônico fundamental. Posteriormente, é considerado um tempo de vida longo do estado eletrônico ${ }^{1}\left(\pi \pi^{*} \mathrm{~L}_{\mathrm{b}}\right)$ onde é atingido o equilíbrio eletrostático soluto-solvente neste estado eletrônico. Esse procedimento é também mostrado nas figuras 8.3c pela convergência dos pontos rômbicos. Dividindo o procedimento iterativo desta forma é possível observar explicitamente a polarização eletrônica correspondente ao estado eletrônico excitado uma vez que ele é populado. Dessa forma, pode-se ver na figura $8.3 \mathrm{c}$ que a polarização eletrônica do estado ${ }^{1}\left(\pi \pi^{*} \mathrm{~L}_{\mathrm{b}}\right)$ produto da interação com a água provoca um aumento de dipolo de 11.1 para $19.8 \mathrm{D}$.

Tanto na condição de equilíbrio eletrostático quanto de desequilíbrio, o primeiro estado eletrônico excitado é o estado ${ }^{1}\left(\pi \pi^{*} \mathrm{~L}_{\mathrm{b}}\right)$. Devido à interação com a água, este estado eletrônico estabiliza consideravelmente, enquanto, o estado ${ }^{1}\left(n \pi^{*}\right)$, que na fase gasosa e na absorção aparecia como primeiro estado excitado, é deslocado para o azul.

As energias de emissão em água, calculadas no nível CASPT2/ANO-L, são mostradas na tabela 8.1. Na condição de desequilíbrio eletrostático soluto-solvente, a energia de emissão correspondente ao estado eletrônico ${ }^{1}\left(\pi \pi^{*} \mathrm{~L}_{\mathrm{b}}\right)$ é de $3.24 \mathrm{eV}$ (383 $\mathrm{nm}$ ). Isso leva a um valor de Stokes shift em água, comparando com a energia de absorção, de $0.92 \mathrm{eV}(85 \mathrm{~nm})$. Considerando agora que o tempo de vida do estado eletrônico excitado ${ }^{1}\left(\pi \pi^{*} \mathrm{~L}_{\mathrm{b}}\right)$ é suficientemente grande para atingir o equilíbrio eletrostático soluto-solvente, a energia de emissão calculada em água é de 2.85 eV (435 $\mathrm{nm})$, também indicado na tabela 8.1. Esse valor está em muito boa concordância com o resultado experimental de Beyhan et al. ${ }^{[243]}$, onde a banda de emissão é observada com máximo próximo de $2.67 \mathrm{eV}(464 \mathrm{~nm})$. Comparando com a energia de absorção, obtémse um valor de Stokes shift em água de $1.31 \mathrm{eV}(134 \mathrm{~nm})$. Esse grande valor do Stokes shift está em boa concordância com o resultado experimental de $1.40 \mathrm{eV}^{[243]}$.

Segundo os resultados mostrados, a interação com o solvente tem grande influência no Stokes shift do sistema molecular daidzein. Consideramos que a grande polarização eletrônica do estado eletrônico excitado ${ }^{1}\left(\pi \pi^{*} \mathrm{~L}_{\mathrm{b}}\right)$, mostrada na figura $8.3 \mathrm{c}$, é a principal causa do grande Stokes shift do daidzein observado experimentalmente, fazendo com que a energia deste estado eletrônico estabilize consideravelmente. 


\subsubsection{Decaimento não fluorescente em acetonitrila}

Como foi comentado, experimentalmente não é observada fluorescência do daidzein em solvente polar aprótico como a acetonitrila. Nesta seção, tentaremos explicar as causas desse decaimento não fluorescente.

Segundo os resultados mostrados na tabela 8.1, o estado eletrônico mais populado nas transições de absorção é o estado ${ }^{1}\left(\pi \pi^{*} \mathrm{~L}_{\mathrm{b}}\right)$. Portanto, usando a geometria de equilíbrio deste estado eletrônico foi considerado o efeito da acetonitrila como solvente nas energias dos estados eletrônicos. Diferente do que acontece em água, quando é usada a acetonitrila o estado eletrônico de menor energia é o estado ${ }^{1}\left(n \pi^{*}\right)$. Como se pode observar na figura $8.3 \mathrm{~d}$, a polarização eletrônica do daidzein no estado eletrônico ${ }^{1}\left(\pi \pi^{*} \mathrm{~L}_{\mathrm{b}}\right)$ produto da interação com a acetonitrila é fraca, provocando uma pequena variação no valor do momento de dipolo de 4.9 para 6.4 D. Por causa disso, o estado eletrônico ${ }^{1}\left(\pi \pi^{*} \mathrm{~L}_{\mathrm{b}}\right)$ não consegue estabilizar o suficiente, ficando menos estável do que o estado ${ }^{1}\left(n \pi^{*}\right)$.

Nessas condições, segundo a regra de Kasha ${ }^{[31]}$, o sistema vai decair por conversão interna do estado ${ }^{1}\left(\pi \pi^{*} \mathrm{~L}_{\mathrm{b}}\right)$ para o estado ${ }^{1}\left(n \pi^{*}\right)$. Portanto, a fim de esclarecer a forma de decaimento não fluorescente do daidzein, considerou-se estudar o intersystem crossing entre o estado eletrônico ${ }^{1}\left(n \pi^{*}\right)$ e os estados tripletos. A geometria de equilíbrio do estado eletrônico ${ }^{1}\left(n \pi^{*}\right)$ foi otimizada no nível CASSCF/ANO-L, obtendo a geometria mostrada na figura 8.2c, onde o chromone e o grupo fenil formam um ângulo de aproximadamente $59.15^{\circ}$. Usando esta geometria foram calculados os valores do acoplamento spin-órbita entre este estado eletrônico e os estados tripletos mais próximos. Estes cálculos foram realizados na fase gasosa e são mostrados na tabela 8.2. O maior valor de SOC é observado com o estado tripleto $3^{3}\left(\pi \pi^{*}\right) \operatorname{com} 42.5 \mathrm{~cm}^{-1}$. Mesmo que a energia deste estado eletrônico apareça $0.2 \mathrm{eV}$ acima da energia do estado singleto, essa ordem deve-se inverter na presença da acetonitrila, onde nós achamos que o estado ${ }^{1}\left(n \pi^{*}\right)$ seja levemente deslocado para o azul e o estado $3^{3}\left(\pi \pi^{*}\right)$ para o vermelho. Os resultados mostrados na tabela 8.2 estão em excelente concordância com a regra de El-Sayed ${ }^{[35]}$, onde é indicado que o intersystem crossing é favorável quando estão envolvidos estados eletrônicos de diferente simetria espacial, como são o estado singleto ${ }^{1}\left(n \pi^{*}\right)$ e os tripletos $1^{3}\left(\pi \pi^{*}\right)$ e $3^{1}\left(\pi \pi^{*}\right)$. O SOC com o estado tripleto $2^{3}\left(n \pi^{*}\right)$ é desprezível. 
Tabela 8.2. Valores calculados do acoplamento spin-órbita e diferenças energéticas entre os estados eletrônicos considerados.

\begin{tabular}{c|c|c|c}
\hline \multicolumn{2}{c|}{ Estado } & $\mathrm{SOC}\left(\mathrm{cm}^{-1}\right)$ & $\Delta E^{\mathrm{a})}(\mathrm{eV})$ \\
\hline \multirow{2}{*}{$\left(n \pi^{*}\right)$} & $1^{3}\left(\pi \pi^{*}\right)$ & 31.4 & -3.64 \\
& $2^{3}\left(n \pi^{*}\right)$ & - & -0.29 \\
& $3^{3}\left(\pi \pi^{*}\right)$ & 42.5 & 0.20 \\
\hline \multicolumn{3}{c}{${ }^{\text {a) }}$ Energias em relação ao estado excitado singleto ${ }^{1}\left(n \pi^{*}\right)}$.
\end{tabular}

O intersystem crossing rate, como foi mostrado no capítulo anterior, e na seção (1.5.2) é proporcional ao acoplamento spin-órbita e ao acoplamento vibracional entre os estados eletrônicos. Lá, para o 1-nitronaftaleno, o acoplamento vibracional foi aproximadamente determinado, mas, de forma geral ele é inversamente proporcional a diferença energética entre os estado eletrônicos. Isto é resumido através do fator de interação spin-órbita $\left(f_{S O}\right)$ :

$$
\mathrm{f}_{\mathrm{SO}}=\frac{\mathrm{SOC}^{2}}{\Delta E^{2}}
$$

onde segundo Turro ${ }^{[213]}$, grandes valores deste fator conduz a grandes valores do intersystem crossing rate. Com tudo isso, podemos concluir que o grande valor do SOC entre os estados eletrônicos ${ }^{1}\left(n \pi^{*}\right)$ e $3^{3}\left(\pi \pi^{*}\right)$ e a pequena diferença energética entre eles pode conduzir a um eficiente intersystem crossing, impedindo assim o decaimento por fluorescência deste sistema molecular em acetonitrila.

\subsection{Conclusões}

Foi estudado de forma teórica a fotofísica do sistema molecular daidzein nos solventes água e acetonitrila. Os espectros de absorção foram corretamente caracterizados em ambos os solventes, mas a desativação eletrônica apresenta diferenças segundo o tipo de solvente usado, como é indicado experimentalmente.

Os resultados obtidos são resumidos nas ilustrações mostradas na figura 8.5. O mecanismo de fluorescência do daidzein em água após a absorção eletrônica é, de forma geral, mostrado na figura 8.5a. O estado eletrônico ${ }^{1}\left(n \pi^{*}\right)$, que na fase gasosa e em acetonitrila é o primeiro estado excitado, é deslocado para o azul produto da interação com a água, enquanto o estado eletrônico ${ }^{1}\left(\pi \pi^{*} L_{b}\right)$ é estabilizado energeticamente. A 
energia de emissão deste estado eletrônico está em excelente concordância com o resultado experimental, da mesma forma que o Stokes shift calculado.

Em acetonitrila o panorama é diferente, como é indicado na figura $8.5 \mathrm{~b}$. O estado eletrônico mais populado nas transições de absorção é o estado ${ }^{1}\left(\pi \pi^{*} \mathrm{~L}_{\mathrm{b}}\right)$. Este estado eletrônico na sua geometria de equilíbrio é pouco solvatado pela acetonitrila, ganhando assim pouca estabilidade energética. De igual forma, o estado eletrônico ${ }^{1}\left(n \pi^{*}\right)$ não é suficientemente deslocado para o azul, permanecendo mais estável energeticamente que o estado ${ }^{1}\left(\pi \pi^{*} \mathrm{~L}_{\mathrm{b}}\right)$. O estado eletrônico ${ }^{1}\left(n \pi^{*}\right)$, que pode ser populado por conversão interna segundo a regra de Kasha, apresenta condições muito favoráveis para um intersystem crossing com o estado tripleto $3^{3}\left(\pi \pi^{*}\right)$. Podemos concluir que o mecanismo de desativação mostrado na figura $8.5 \mathrm{~b}$ explica o decaimento não fluorescente do daidzein em acetonitrila observado experimentalmente. O mecanismo de desativação dos estados tripletos pode ser posteriormente objeto de estudo como continuidade destes resultados.

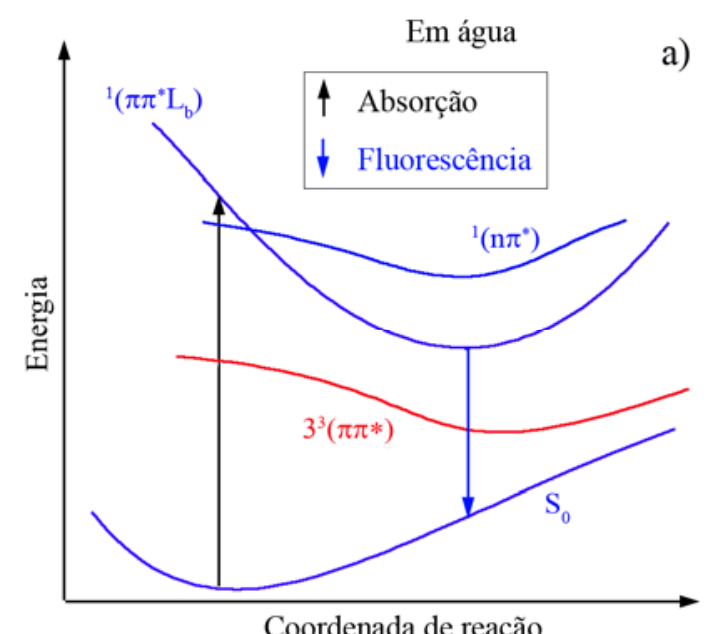

Coordenada de reação

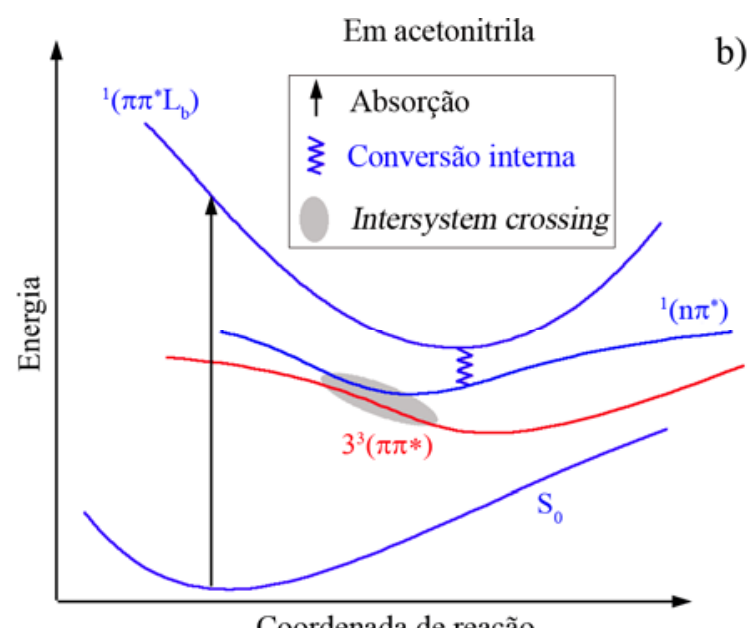

Coordenada de reação

Figura 8.5. Perfis energéticos esquemáticos dos estados eletrônicos e processos envolvidos na fotofísica do daidzein em água (a) e em acetonitrila (b).

È interessante observar que essas mudanças na evolução e decaimento dos estados eletrônicos excitados, em função do tipo de solvente, foram também observadas no sistema molecular 5-fluorouracil. Neste sistema é observado experimentalmente que a taxa de fluorescência é muito menor em acetonitrila que em água. Os estudos teóricos de Improta et al. ${ }^{[248,249]}$ indicam, de forma geral, que a estabilidade dos estados eletrônicos $n \pi^{*}$ (escuro) e $\pi \pi^{*}$ (brilhante) em acetonitrila são comparáveis, estando 
muito próximo um estado do outro. Dessa forma, além da fluorescência do estado $\pi \pi^{*}$, aparece um outro mecanismo de desativação eletrônica a traves de uma intersecção cônica entre estes dois estados. Isso não é observado em água, onde o estado $n \pi *$ é bem mais instável que o $\pi \pi^{*}$ devido à maior solvatação destes estados eletrônicos, onde eles são deslocados respectivamente para o azul e para o vermelho. 


\section{Conclusões gerais}

Nesta tese foram estudados vários sistemas moleculares, tanto em fase gasosa como na presença de solvente. Na fase gasosa foi estudada s superfície de energia potencial do sistema molecular $\mathrm{HSO}_{2}$ e a influência da energia rotacional na reatividade da reação $\mathrm{OH}+\mathrm{SO}$. O estudo do efeito do solvente na reatividade deste sistema molecular ficou pendente para trabalhos futuros devido aos inconvenientes comentados na introdução da tese. Por outra parte, foi estudado o efeito do solvente nas propriedades e na evolução dos estados eletrônicos excitados dos seguintes sistemas moleculares: acetona, 1nitronaftaleno e daidzein. Foram obtidos resultados interessantes que serão comentados a seguir.

Em relação ao sistema molecular $\mathrm{HSO}_{2}$, temos que no estudo da superfície de energia potencial foi feita uma caracterização detalhada de cada uma das estruturas estacionárias com um nível de cálculo suficientemente alto. Assim, foi elaborado o diagrama energético que representa a topologia geral da superfície de energia potencial e oferece informação relevante dos diferentes caminhos de reação. A conexão entre as regiões mais energética e menos energética foi estabelecida através de um estado de transição que não tinha sido reportado anteriormente. Os resultados obtidos neste trabalho vão ser usados para recalibrar algumas regiões da superfície de energia potencial global DMBE deste sistema molecular. Também foi estudado o efeito da energia rotacional na reação $\mathrm{OH}+\mathrm{SO}$, para isto, foi feito um estudo de trajetórias quaseclássicas usando a superfície de energia potencial global DMBE publicada anteriormente na literatura. Esta superfície de energia potencial, na região de menores 
energias, concorda muito bem com a caracterização da superfície de energia potencial comentada anteriormente. Os resultados mostram uma reação de tipo captura, onde a energia rotacional depositada, tanto no $\mathrm{OH}$ quanto no $\mathrm{SO}$, contribui à tendência negativa da constante de velocidade de reação com a temperatura. A constante de velocidade de reação média obtida concorda razoavelmente bem com os dados experimentais para temperaturas menores que $500 \mathrm{~K}$.

Nos estudos dedicados ao efeito de solvente nas propriedades espectroscópicas dos sistemas moleculares foi estudado, principalmente, o efeito de solvente nas propriedades dos estados eletrônicos excitados usando a metodologia sequencial QM/MM.

No trabalho apresentado da emissão $n \leftarrow \pi^{*}$ da acetona em água foi considerada a importância da polarização eletrônica do estado eletrônico excitado no deslocamento espectral da banda de emissão. Obtendo-se pouca dependência do deslocamento espectral com a polarização eletrônica do estado excitado. O espectro de emissão obtido em água apresenta um deslocamento muito pequeno comparado com a fase gasosa, concordando com as evidências experimentais e outros resultados teóricos. Para complementar o estudo da acetona, também foi analisado o deslocamento da banda de absorção $n \rightarrow \pi^{*}$, resultando em um deslocamento espectral em concordância com os resultados teóricos e experimentais anteriores. Foram analisadas também as ligações de hidrogênio em ambos estados eletrônicos, obtendo-se resultados em excelente concordância com os tipos de transições estudadas e com valores anteriormente reportados.

No estudo do sistema molecular 1-nitronaftaleno foi esclarecido teoricamente o mecanismo de desativação eletrônica, além de serem identificados os estados eletrônicos que participam. A rápida mudança de estado eletrônico para os estados tripletos, após a transição de absorção, foi explicada por um eficiente intersystem crossing que pode acontecer ao longo do caminho de relaxação do estado excitado $\mathrm{S}_{1}$. Ao longo deste caminho de relaxação foi calculado o acoplamento spin-órbita entre os estados eletrônicos excitados $\mathrm{S}_{1}$ e $\mathrm{T}_{2}$, e foi aproximadamente estimado o intersystem crossing rate entre estes estados, mostrando ser altamente eficiente. O mecanismo de desativação proposto é capas de reproduzir os espectros de absorção transitória observados experimentalmente. Nos cálculos destes espectros, assim como nos de absorção e fosforescência, foi considerado o efeito do solvente usando a metodologia sequencial QM/MM. Os resultados obtidos concordam muito bem com os resultados experimentais. 
No sistema molecular daidzein foi estudada a fotofísica do sistema na presença de solventes, mostrando diferencias segundo o tipo de solvente utilizado. Em água foi estudado teoricamente o Stokes shift, a fim de esclarecer as causas que provocam o grande valor experimentalmente observado. Os espectros de absorção e emissão foram corretamente calculados, oferecendo resultados em excelente concordância com o experimento. A grande polarização eletrônica observada no estado eletrônico fluorescente, produto da interação com a água, indica ser a principal causa do grande valor do Stokes shift observado experimentalmente neste sistema molecular. Em solvente polar aprótico como a acetonitrila, foi observado um decaimento não fluorescente, produzido por um mecanismo de ISC bastante eficiente com os estados tripletos. A diferença entre um caso e o outro deve-se aos diferentes graus de solvatação que produz cada solvente nos estados eletrônicos excitados. 
144 Conclusões gerais 


\section{Publicações relacionadas}

1 A quasiclassical trajectory study of the $\mathrm{OH}+\mathrm{SO}$ reaction: The role of rotational energy. M. Y. Ballester, Y. Orozco-Gonzalez, J. D. Garrido, H. F. Dos Santos, J. Chem. Phys. 132 (2010) 044310.

2 A CASPT2 study of the potential energy surface of the $\mathrm{HSO}_{2}$ system, J. D. Garrido, M. Y. Ballester, Y. Orozco-Gonzalez, S. Canuto, J. Phys. Chem. A, 115 (9) (2011) 1453.

3 Excited state electronic polarization and reappraisal of the $n \leftarrow \pi^{*}$ emission of acetone in water, Y. Orozco-Gonzalez, K. Coutinho, S. Canuto, Chem. Phys. Lett. 499 (2010) 108.

4 Theoretical study of the absorption and nonradiative deactivation of 1 Nitronaphtalene in the low-lying singlet and triplet excited states including methanol and ethanol solvent effects, Y. Orozco-Gonzalez, K. Coutinho, J. Peon, S. Canuto, J. Chem. Phys. 137 (2012) 054307.

5 Solvent effect on the Stokes shift and on the nonradiative decay of the daidzein molecular system. Y. Orozco-Gonzalez, C. Bistafa, S. Canuto, em processo de submissão. 


\section{Referências}

[1] M. Grätzel J. Photochem. Photobiol. C: Photochem. Rev. 2003, 4, 145.

[2] P. Agostinis, K. Berg, K. A. Cengel, T. H. Foster, A. W. Girotti, S. O. Gollnick, S. M. Hahn, M. R. Hamblin, A. Juzeniene, D. Kessel, M. Korbelik, J. Moan, P. Mroz, D. Nowis, J. Piette, B. C. Wilson, J. Golab CA Cancer J. Clin. 2011, 61, 250.

[3] J. F. Callan, A. P. de Silva, D. C. Magri Tetrahedron. 2005, 61, 8551.

[4] J. C. Scaiano, C. Aliaga, M. N. Chretien, M. Frenette, K. S. Focsaneanu, L. Mikelsons Pure Appl. Chem. 2005, 77, 1009.

[5] S. Weiss Science. 1999, 283, 1676.

[6] L. Zang, R. C. Liu, M. W. Holman, K. T. Nguyen, D. M. Adams J. Am. Chem. Soc. 2002, 124, 10640 .

[7] L. Onsager J. Am. Chem. Soc. 1936, 58, 1486.

[8] J. G. Kirkwood J. Chem. Phys. 1934, 2, 351.

[9] B. Mennucci, M. Cossi, J. Tomasi J. Chem. Phys. 1995, 102, 6837.

[10] J. L. Rivail, D. Rinaldi Chem. Phys. 1976, 18, 233.

[11] S. Miertus, E. Scrocco, J. Tomasi Chem. Phys. 1981, 55, 117.

[12] R. Car, M. Parrinello Phys. Rev. Lett. 1985, 55, 2471.

[13] R. N. Barnett, U. Landman, A. Nitzan, G. Rajagopal J. Chem. Phys. 1991, 94, 608. 
[14] K. Coutinho, S. Canuto Adv. Quant. Chem. 1997, 28, 89-105.

[15] C. L. Zhou, K. Sendt, B. S. Haynes J. Phys. Chem. A. 2009, 113, 2975.

[16] M. Born, R. Oppenheimer Annalen Der Physik. 1927, 84, 0457.

[17] M. Born, K. Huang, Dynamical theory of crystal lattices, Apêndice VII, Clarendon, Oxford, 1954.

[18] D. C. Young, Computational chemistry: a practical guide for applying techniques to real world problems, Wiley, New York, Chichester, 2001.

[19] M. Klessinger, J. Michl, Excited states and photochemistry of organic molecules, VCH, New York, Cambridge, 1995.

[20] H. Zimmerma J. Am. Chem. Soc. 1966, 88, 1566.

[21] J. Michl Mol. Photochem. 1972, 4, 243.

[22] A. S. Davydov, T. D. Haar, Quantum mechanics, Pergamon, Oxford, 1965.

[23] V. Fock Zeitschrift Fur Physik. 1930, 61, 126.

[24] A. Szabo, N. S. Ostlund, Modern quantum chemistry: introduction to advanced electronic structure theory, Dover Publications, Mineola, N.Y., 1996.

[25] I. N. Levine, Quantum chemistry, Pearson Prentice Hall, Upper saddle river, N.J., 2009.

[26] T. Helgaker, P. Jørgensen, J. Olsen, Molecular electronic-structure theory, John Wiley \& Sons, Chichester, 2000.

[27] M. Head-Gordon, R. J. Rico, M. Oumi, T. J. Lee Chem. Phys. Lett. 1994, 219, 21.

[28] B. O. Roos, P. R. Taylor, P. E. M. Siegbahn Chem. Phys. 1980, 48, 157.

[29] C. Moller, M. S. Plesset Phys. Rev. 1934, 46, 0618.

[30] K. Andersson, P. A. Malmqvist, B. O. Roos J. Chem. Phys. 1992, 96, 1218.

[31] M. Kasha Discuss. Faraday Soc. 1950, 14.

[32] W. Siebrand J. Chem. Phys. 1967, 46, 440.

[33] R. McWeeny, Methods of molecular quantum mechanics, Academic Press, London, San Diego, 1989.

[34] B. A. Hess, C. M. Marian, U. Wahlgren, O. Gropen Chem. Phys. Lett. 1996, 251, 365.

[35] M. A. El-Sayed J. Chem. Phys. 1963, 38, 2834. 
[36] A. J. C. Varandas in Conical Intersections Electronic Structure, Dynamics and Spectroscopy, Vol. Modeling and interpolation of global multi-sheeted potential energy surfaces, World Scientific, 2004, pp.205.

[37] D. L. Bunker Meth. Comput. Phys. 1971, 10, 287.

[38] A. J. C. Varandas Int. Rev. Phys. Chem. 2000, 19, 199.

[39] J. N. Murrell, Molecular potential energy functions, Wiley, Chichester, 1984.

[40] A. J. C. Varandas Adv. Chem. Phys. 1988, 74, 255.

[41] A. J. C. Varandas in Reaction and Molecular Dynamics, Vol. 75 (Eds.: A. Laganá, A. Riganelli), Springer, Berlin, 2000.

[42] A. J. C. Varandas Mol. Phys. 1984, 53, 1303.

[43] A. J. C. Varandas Chem. Phys. Lett. 1992, 194, 333.

[44] A. J. C. Varandas J. Mol. Struct.: THEOCHEM. 1985, 120, 401.

[45] A. J. C. Varandas, J. D. da Silva J. Chem. Soc., Faraday Trans. 2. 1986, 82, 593.

[46] R. D. Levine, Molecular reaction dynamics, Cambridge University Press, Cambridge, 2009.

[47] A. J. C. Varandas Chem. Phys. Lett. 1994, 225, 18.

[48] M. Karplus, R. D. Sharma J. Chem. Phys. 1965, 43, 3259.

[49] M. Karplus, R. D. Sharma, R. N. Porter J. Chem. Phys. 1964, 40, 2033.

[50] M. Karplus, K. T. Tang Discuss. Faraday Soc. 1967, 56.

[51] H. Golstein, Classical Mechanics, Addison-Wesley, London, 1950.

[52] P. J. S. B. Caridade, M. Betancourt, J. D. Garrido, A. J. C. Varandas J. Phys. Chem. A. 2001, 105, 7435.

[53] P. J. S. B. Caridade, L. Zhang, J. D. Garrido, A. J. C. Varandas J. Phys. Chem. A. 2001, 105, 4395.

[54] R. L. LeRoy J. Phys. Chem. 1969, 73, 4338.

[55] J. D. Jackson, Classical electrodynamics, Wiley, New York, Chichester, 1999.

[56] J. O. Hirschfelder, R. B. Bird, C. F. Curtiss, Molecular Theory of Gases and Liquids., Chapman \& Hall ; New York : John Wiley \& Sons, London, 1954.

[57] A. J. C. Varandas, Proceedings of the Conferencias Plenarias de la XXIII Reunion bienal de Química, Universidade de Salamanca, Salamanca, Spain, 1990.

[58] M. Y. Ballester, A. J. C. Varandas Chem. Phys. Lett. 2007, 433, 279. 
[59] M. Y. Ballester, A. J. C. Varandas Int. J. Chem. Kinet. 2008, 40, 533.

[60] W. L. Hase, MERCURY: a general Monte Carlo classical trajectory computer program, QCPE \# 453, an updated version of this code is VENUS 96; W. L. Hase, R. J. Duchovic, X. Hu, A. Komornik, K. F. Lim, D. H. Lu, G. H. Peslherbe, K. N. Swamy, S. R. van de Linde, A. J. C. Varandas, H. Wang, R. J. Wolf, QCPE Bull. 1996, 16, 43.

[61] W. L. Hase, D. G. Buckowski Chem. Phys. Lett. 1980, 74, 284.

[62] S. Califano, Vibrational states, Wiley-Interscience, London, 1976.

[63] I. N. Levine, Molecular spectroscopy, Wiley-Interscience, New York, 1975.

[64] S. Chapman, D. L. Bunker J. Chem. Phys. 1975, 62, 2890.

[65] C. S. Sloane, W. L. Hase J. Chem. Phys. 1977, 66, 1523.

[66] E. B. Wilson, P. C. Cross, J. C. Decius, Molecular Vibrations. The theory of infrared and Raman vibrational spectra., McGraw-Hill Book Co., New York, 1955.

[67] N. Metropolis, A. W. Rosenbluth, M. N. Rosenbluth, A. H. Teller, E. Teller $J$. Chem. Phys. 1953, 21, 1087.

[68] M. P. Allen, D. J. Tildesley, Computer simulation of liquids, Clarendon Press, Oxford, 1987.

[69] K. Coutinho, S. Canuto, DICE: a Monte Carlo program for molecular liquid simulations, University of São Paulo, São Paulo, 2009.

[70] K. Coutinho, S. Canuto, M. C. Zerner J. Chem. Phys. 2000, 112, 9874.

[71] C. Chatfield, The analysis of time series: An introduction, Chapman and Hall, London, 1996.

[72] K. Coutinho, Modelo discreto de solvente. Solvatocromismo no espectro de absorção molecular, Universidade de São Paulo, São Paulo, 1997.

[73] E. E. Dahlke, D. G. Truhlar J. Chem. Theory Comput. 2007, 3, 46.

[74] N. A. McDonald, H. A. Carlson, W. L. Jorgensen J. Phys. Org. Chem. 1997, 10, 563.

[75] S. Nakagawa, P. Mark, H. Agren J. Chem. Theory Comput. 2007, 3, 1947.

[76] J. Kongsted, A. Osted, K. V. Mikkelsen, O. Christiansen Mol. Phys. 2002, 100, 1813.

[77] M. E. Martin, M. L. Sanchez, F. J. O. del Valle, M. A. Aguilar J. Chem. Phys. 2000, 113, 6308 .

[78] H. C. Georg, K. Coutinho, S. Canuto Chem. Phys. Lett. 2006, 429, 119.

[79] C. M. Breneman, K. B. Wiberg J. Comput. Chem. 1990, 11, 361. 
[80] K. Coutinho, H. C. Georg, T. L. Fonseca, V. Ludwig, S. Canuto Chem. Phys. Lett. 2007, 437, 148.

[81] H. C. Georg, K. Coutinho, S. Canuto J. Chem. Phys. 2007, 126, 034507.

[82] S. Canuto, K. Coutinho, D. Trzesniak Adv. Quantum Chem. 2002, 41, 161.

[83] R. P. Wayne, Chemistry of atmospheres: an introduction to the chemistry of the atmospheres of earth, the planets, and their satellites, Oxford University Press, Oxford, 2000.

[84] B. J. Finlayson-Pitts, J. N. Pitts, Chemistry of the upper and lower atmosphere: theory, experiments, and applications, Academic Press, San Diego, 2000.

[85] P. Warneck, Chemistry of the natural atmosphere, Academic Press, San Diego, California, 2000.

[86] C. A. Mcdowell, F. G. Herring, J. C. Tait J. Chem. Phys. 1975, 63, 3278.

[87] D. Binns, P. Marshall J. Chem. Phys. 1991, 95, 4940.

[88] V. R. Morris, W. M. Jackson Chem. Phys. Lett. 1994, 223, 445.

[89] D. Laakso, C. E. Smith, A. Goumri, J. D. R. Rocha, P. Marshall Chem. Phys. Lett. 1994, 227, 377.

[90] A. Goumri, J. D. R. Rocha, P. Marshall J. Phys. Chem. 1995, 99, 10834.

[91] A. J. Frank, M. Sadilek, J. G. Ferrier, F. Turecek J. Am. Chem. Soc. 1996, 118, 11321.

[92] J.-X. Qi, W.-Q. Deng, K.-L. Han, G.-Z. He J. Chem. Soc., Faraday Trans. 1997, 93, 25.

[93] A. J. Frank, M. Sadilek, J. G. Ferrier, F. Turecek J. Am. Chem. Soc. 1997, 119, 12343.

[94] A. Goumri, J. D. R. Rocha, D. Laakso, C. E. Smith, P. Marshall J. Phys. Chem. A. 1999, 103, 11328.

[95] E. Isoniemi, L. Khriachtchev, J. Lundell, M. Rasanen J. Mol. Struct.: THEOCHEM. 2001, 563, 261.

[96] E. Isoniemi, L. Khriachtchev, J. Lundell, M. Rasanen Phys. Chem. Chem. Phys. 2002, 4, 1549.

[97] P. A. Denis, O. N. Ventura Chem. Phys. Lett. 2001, 344, 221.

[98] M. Y. Ballester, A. J. C. Varandas Phys. Chem. Chem. Phys. 2005, 7, 2305.

[99] M. Nicolet Rev. Geophys. 1975, 13, 593.

[100] R. Atkinson, A. C. Lloyd J. Phys. Chem. Ref. Data. 1984, 13, 315. 
[101] J. W. E. Wilson J. Phys. Chem. Ref. Data. 1972, 1, 535.

[102] K. Schofield J. Phys. Chem. Ref. Data. 1973, 2, 25.

[103] M. U. Alzueta, R. Bilbao, P. Glarborg Comb. Flame. 2001, 127, 2234.

[104] R. W. Fair, B. A. Thrush Trans. Faraday Soc. 1969, 65, 1557.

[105] J. L. Jourdain, G. Lebras, J. Combourieu Int. J. Chem. Kinet. 1979, 11, 569.

[106] M. A. Blitz, K. W. McKee, M. J. Pilling P. Combust. Inst. 2000, 28, 2491.

[107] M. Jorfi, P. Honvault, P. Halvick, S. Y. Lin, H. Guo Chem. Phys. Lett. 2008, 462, 53.

[108] M. Jorfi, P. Honvault, P. Halvick Chem. Phys. Lett. 2009, 471, 65.

[109] A. Zanchet, P. Halvick, J. C. Rayez, B. Bussery-Honvault, P. Honvault J. Chem. Phys. 2007, 126, 184308.

[110] M. Y. Ballester, A. J. C. Varandas Int. J. Chem. Kinet. 2009, 41, 455.

[111] M. Y. Ballester, P. J. S. B. Caridade, A. J. C. Varandas Chem. Phys. Lett. 2007, 439, 301.

[112] E. Martinez-Nunez, A. J. C. Varandas J. Phys. Chem. A. 2001, 105, 5923.

[113] A. J. C. Varandas, A. I. Voronin Mol. Phys. 1995, 85, 497.

[114] A. J. C. Varandas, A. I. Voronin Chem. Phys. 1995, 194, 91.

[115] A. J. C. Varandas, S. P. J. Rodrigues Spectrochim. Acta Part A. 2002, 58, 629.

[116] M. R. Pastrana, L. A. M. Quintales, J. Brandao, A. J. C. Varandas J. Phys. Chem. 1990, 94, 8073.

[117] M. Y. Ballester, Y. O. Guerrero, J. D. Garrido Int. J. Quantum Chem. 2008, 108, 1705 .

[118] J. D. Garrido, M. A. C. Nascimento, M. Y. Ballester Int. J. Quantum Chem. 2010, 110,549 .

[119] D. G. Truhlar J. Chem. Phys. 1972, 56, 3189.

[120] J. T. Muckerma, M. D. Newton J. Chem. Phys. 1972, 56, 3191.

[121] K. P. Huber, G. Herzberg Constants of diatomics molecules, Van Nostrand, Princeton. 1979.

[122] A. J. C. Varandas, L. Zhang Chem. Phys. Lett. 2001, 340, 62.

[123] M. T. Bell, T. P. Softley Mol. Phys. 2009, 107, 99. 
[124] F. J. Aoiz, V. J. Herrero, V. S. Rabanos J. Chem. Phys. 1991, 94, 7991.

[125] D. C. Clary Mol. Phys. 1984, 53, 3.

[126] M. Y. Ballester, Y. Orozco-Gonzalez, J. D. Garrido, H. F. Dos Santos J. Chem. Phys. 2010, 132, 044310.

[127] R. J. Boyd, A. Gupta, R. F. Langler, S. P. Lownie, J. A. Pincock Can. J. Chem. $1980,58,331$.

[128] A. Hinchliffe J. Mol. Struct.: THEOCHEM. 1981, 71, 349.

[129] D. Laakso, P. Marshall J. Phys. Chem. 1992, 96, 2471.

[130] Y. Drozdova, R. Steudel, R. H. Hertwig, W. Koch, T. Steiger J. Phys. Chem. A. 1998, 102, 990.

[131] M. L. McKee, P. H. Wine J. Am. Chem. Soc. 2001, 123, 2344.

[132] B. S. Wang, H. Hou Chem. Phys. Lett. 2005, 410, 235.

[133] B. Napolion, J. D. Watts Chem. Phys. Lett. 2006, 421, 562.

[134] M. Wierzejewska, A. Olbert-Majkut J. Phys. Chem. A. 2007, 111, 2790.

[135] A. J. Midey, A. A. Viggiano J. Phys. Chem. A. 2007, 111, 1852.

[136] S. E. Wheeler, H. F. Schaefer J. Phys. Chem. A. 2009, 113, 6779.

[137] S. M. Resende, F. R. Ornellas Phys. Chem. Chem. Phys. 2003, 5, 4617.

[138] D. J. Grant, D. A. Dixon, J. S. Francisco, D. Feller, K. A. Peterson J. Phys. Chem. A. 2009, 113, 11343 .

[139] H.-J. Werner, P. J. Knowles, G. Knizia, F. R. Manby, M. Schutz, et al., MOLPRO, version 2010.1, a package of ab initio programs, Cardiff, UK, 2010.

[140] J. M. L. Martin J. Chem. Phys. 1998, 108, 2791.

[141] J. M. L. Martin, O. Uzan Chem. Phys. Lett. 1998, 282, 16.

[142] C. W. Bauschlicher, A. Ricca J. Phys. Chem. A. 1998, 102, 8044.

[143] C. W. Bauschlicher, H. Partridge Chem. Phys. Lett. 1995, 240, 533.

[144] T. H. Dunning, K. A. Peterson, A. K. Wilson J. Chem. Phys. 2001, 114, 9244.

[145] A. J. C. Varandas J. Phys. Chem. A. 2010, 114, 8505.

[146] K. A. Peterson, D. E. Woon, J. T. H. Dunning J. Chem. Phys. 1994, 100, 7410.

[147] S. Tenno, F. Hirata, S. Kato J. Chem. Phys. 1994, 100, 7443. 
[148] M. Cossi, V. Barone J. Chem. Phys. 2000, 112, 2427.

[149] L. Bernasconi, M. Sprik, J. Hutter J. Chem. Phys. 2003, 119, 12417.

[150] J. L. Gao J. Am. Chem. Soc. 1994, 116, 9324.

[151] K. Coutinho, S. Canuto J. Mol. Struct.: THEOCHEM. 2003, 632, 235.

[152] K. Aidas, J. Kongsted, A. Osted, K. V. Mikkelsen, O. Christiansen J. Phys. Chem. A. 2005, 109, 8001 .

[153] U. F. Röhrig, I. Frank, J. Hutter, A. Laio, J. VandeVondele, U. Rothlisberger Chem. Phys. Chem. 2003, 4, 1177.

[154] A. Ohrn, G. Karlstrom Theor. Chem. Acc. 2007, 117, 441.

[155] M. Olivucci, Computational photochemistry, Elsevier, Amsterdam, Boston, 2005.

[156] V. Ludwig, M. S. do Amaral, Z. M. da Costa, A. C. Borin, S. Canuto, L. SerranoAndres Chem. Phys. Lett. 2008, 463, 201.

[157] M. Barbatti, H. Lischka J. Am. Chem. Soc. 2008, 130, 6831.

[158] P. Suppan, N. Ghoneim, Solvatochromism, Royal Society of Chemistry Information Services, Cambridge, 1997.

[159] Osulliva.M, A. C. Testa J. Am. Chem. Soc. 1970, 92, 5842.

[160] W. R. Rocha, K. Coutinho, W. B. de Almeida, S. Canuto Chem. Phys. Lett. 2001, $335,127$.

[161] W. L. Jorgensen, J. Chandrasekhar, J. D. Madura, R. W. Impey, M. L. Klein J. Chem. Phys. 1983, 79, 926.

[162] K. Coutinho, S. Canuto J. Chem. Phys. 2000, 113, 9132.

[163] D. W. Liao, A. M. Mebel, M. Hayashi, Y. J. Shiu, Y. T. Chen, S. H. Lin J. Chem. Phys. 1999, 111, 205.

[164] M. J. Frisch, et. al., Gaussian 03, Revision D.01, Gaussian, Inc., Wallingford, CT, 2004.

[165] D. E. Woon, T. H. Dunning J. Chem. Phys. 1993, 98, 1358.

[166] R. Peter, H. Dreizler Z Naturforsch A. 1965, 20, 301.

[167] T. Malaspina, K. Coutinho, S. Canuto J. Chem. Phys. 2002, 117, 1692.

[168] K. Coutinho, N. Saavedra, S. Canuto J. Mol. Struct.: THEOCHEM. 1999, 466, 69.

[169] N. S. Bayliss, E. G. Mcrae J. Phys. Chem. 1954, 58, 1006.

[170] N. S. Bayliss, G. Wills-Johnson Spectrochim. Acta Part A. 1968, $24,551$. 
[171] W. P. Hayes, C. J. Timmons Spectrochim. Acta. 1965, 21, 529.

[172] J. Heicklen, W. A. Noyes J. Am. Chem. Soc. 1959, 81, 3858.

[173] G. D. Renkes, F. S. Wettack J. Am. Chem. Soc. 1969, 91, 7514.

[174] R. F. Borkman, D. R. Kearns J. Chem. Phys. 1966, 44, 945.

[175] B. Rusakowicz, A. C. Testa Spectrochim. Acta Part A. 1971, 27, 787.

[176] R. W. Anderson, Hochstra.Rm, H. Lutz, G. W. Scott Chem. Phys. Lett. 1974, 28, 153.

[177] K. Hamanoue, S. Hirayama, T. Nakayama, H. Teranishi J. Phys. Chem. 1980, 84, 2074.

[178] C. Capellos, G. Porter J. Chem. Soc., Faraday Trans. 2. 1974, 70, 1159.

[179] R. Hurley, A. C. Testa J. Am. Chem. Soc. 1968, 90, 1949.

[180] R. Morales-Cueto, M. Esquivelzeta-Rabell, J. Saucedo-Zugazagoitia, J. Peon J. Phys. Chem. A. 2007, 111, 552.

[181] Y. He, A. Gahlmann, J. S. Feenstra, S. T. Park, A. H. Zewail Chem. Asian J. 2006, 1, 56.

[182] K. Hamanoue, T. Nakayama, K. Kajiwara, S. Yamanaka, K. Ushida J. Chem. Soc., Faraday Trans. 1992, 88, 3145.

[183] K. Fukuhara, M. Kurihara, N. Miyata J. Am. Chem. Soc. 2001, 123, 8662.

[184] J. S. Zugazagoitia, C. X. Almora-Diaz, J. Peon J. Phys. Chem. A. 2008, 112, 358.

[185] J. J. Mikula, R. W. Anderson, L. E. Harris, E. W. Stuebing J. Mol. Spectrosc. 1972, 42, 350 .

[186] T. Fournier, S. M. Tavender, A. W. Parker, G. D. Scholes, D. Phillips J. Phys. Chem. A. 1997, 101, 5320.

[187] J. S. Zugazagoitia, E. Collado-Fregoso, E. F. Plaza-Medina, J. Peon J. Phys. Chem. A. 2009, 113, 805.

[188] C. Reichardt, R. A. Vogt, E. Crespo-Hernandez J. Chem. Phys. 2009, 131, 0224518 .

[189] D. Beljonne, Z. Shuai, G. Pourtois, J. L. Bredas J. Phys. Chem. A. 2001, 105, 3899.

[190] S. P. McGlynn, T. Azumi, M. Kinoshita, Molecular spectroscopy of the triplet state, Prentice-Hall, Englewood Cliffs, N.J., 1969.

[191] S. Shaik, N. D. Epiotis J. Am. Chem. Soc. 1978, 100, 18. 
[192] S. S. Shaik, N. D. Epiotis J. Am. Chem. Soc. 1980, 102, 122.

[193] M. D. Su J. Phys. Chem. 1996, 100, 4339.

[194] P. O. Widmark, P. A. Malmqvist, B. O. Roos Theor. Chim. Acta. 1990, 77, 291.

[195] P. O. Widmark, B. Joakim, Persson, B. O. Roos Theor. Chim. Acta. 1991, 79, 419.

[196] K. Hirao, Recent advances in multireference methods, World Scientific, Singapore; River Edge, NJ, 1999.

[197] P. A. Malmqvist, B. O. Roos Chem. Phys. Lett. 1989, 155, 189.

[198] J. Tatchen, C. M. Marian Chem. Phys. Lett. 1999, 313, 351.

[199] D. Danovich, C. M. Marian, T. Neuheuser, S. D. Peyerimhoff, S. Shaik J. Phys. Chem. A. 1998, 102, 5923.

[200] M. Merchan, L. Serrano-Andres, M. A. Robb, L. Blancafort J. Am. Chem. Soc. 2005, 127, 1820 .

[201] G. Karlström, R. Lindh, P.-Å. Malmqvist, B. O. Roos, U. Ryde, V. Veryazov, P. O. Widmark, M. Cossi, B. Schimmelpfennig, P. Neogrady, L. Seijo, MOLCAS: a program package for computational chemistry, Lund University, Lund, 2003.

[202] R. A. Marcus Rev. Mod. Phys. 1993, 65, 599.

[203] K. V. Mikkelsen, M. A. Ratner Chem. Rev. 1987, 87, 113.

[204] V. Balzani, A. Juris, M. Venturi, S. Campagna, S. Serroni Chem. Rev. 1996, 96, 759.

[205] Y. Orozco-Gonzalez, K. Coutinho, S. Canuto Chem. Phys. Lett. 2010, 499, 108.

[206] R. C. Barreto, K. Coutinho, H. C. Georg, S. Canuto Phys. Chem. Chem. Phys. 2009, 11, 1388.

[207] W. L. Jorgensen, D. L. Severance J. Am. Chem. Soc. 1990, 112, 4768.

[208] M. L. P. Price, D. Ostrovsky, W. L. Jorgensen J. Comput. Chem. 2001, 22, 1340.

[209] W. L. Jorgensen J. Phys. Chem. 1986, 90, 1276.

[210] M. G. Martin, J. I. Siepmann J. Phys. Chem. B. 1998, 102, 2569.

[211] E. Cances, B. Mennucci, J. Tomasi J. Chem. Phys. 1997, 107, 3032.

[212] A. B. Zahlan, G. M. Androes, G. M. Hameka, F. M. Heineken, C. A. Hutchison, G. W. J. Robinson, J. H. van der Waals, The Triplet State, Cambridge University Press, Cambridge, 1967. 
[213] N. J. Turro, Modern molecular photochemistry, University Science Books, Sausalito, CA, 1991.

[214] M. Takezaki, N. Hirota, M. Terazima J. Chem. Phys. 1998, 108, 4685.

[215] J. Quenneville J. Phys. Chem. A. 2011, 115, 12286.

[216] K. Shimoi, S. Masuda, B. Shen, M. Furugori, N. Kinae Mutation Research/Fundamental and Molecular Mechanisms of Mutagenesis. 1996, 350, 153.

[217] P. Knekt, R. Jarvinen, R. Seppanen, M. Heliovaara, L. Teppo, E. Pukkala, A. Aromaa Am. J. Epidemiol. 1997, 146, 223.

[218] A. Monasterio, M. C. Urdaci, I. V. Pinchuk, N. Lopez-Moratalla, J. J. MartinezIrujo Nutr. Cancer. 2004, 50, 90.

[219] J. Terao, Y. Kawai, K. Murcita Asia Pac. J. Clin. Nutr. 2008, 17, 291.

[220] S. Renaud, M. Delorgeril Lancet. 1992, 339, 1523.

[221] H. Tunstall-Pedoe, K. Kuulasmaa, M. Mahonen, H. Tolonen, E. Ruokokoski, P. Amouyel, W. M. Project Lancet. 1999, 353, 1547.

[222] S. Rosenkranz, D. Knirel, H. Dietrich, M. Flesch, E. Erdmann, M. Böhm FASEB J. 2002, 16, 1958.

[223] M. H. Criqui, B. L. Ringel Lancet. 1994, 344, 1719.

[224] M. Gronbaek, A. Deis, T. I. A. Sorensen, U. Becker, P. Schnohr, G. Jensen Br. Med. J. 1995, 310, 1165.

[225] A. L. Klatsky, M. A. Armstrong, G. D. Friedman Am. J. Cardiol. 1997, 80, 416.

[226] M. Gronbaek, U. Becker, D. Johansen, A. Gottschau, P. Schnohr, H. O. Hein, G. Jensen, T. I. A. Sorensen Ann. Intern. Med. 2000, 133, 411.

[227] P. K. Sengupta, M. Kasha Chem. Phys. Lett. 1979, 68, 382.

[228] O. S. Wolfbeis, R. Schipfer Photochem. Photobiol. 1981, 34, 567.

[229] O. S. Wolfbeis, E. Furlinger, H. C. Jha, F. Zilliken Z. Naturforsch. 1984, 39b, 238.

[230] M. Itoh, K. Hasegawa, Y. Fujiwara J. Am. Chem. Soc. 1986, 108, 5853.

[231] D. Mcmorrow, M. Kasha J. Phys. Chem. 1984, 88, 2235.

[232] M. Sarkar, J. Guha Ray, P. K. Sengupta J. Photochem. Photobiol. A. 1996, 95, 157.

[233] B. J. Schwartz, L. A. Peteanu, C. B. Harris J. Phys. Chem. 1992, 96, 3591. 
[234] S. Ameer-Beg, S. M. Ormson, R. G. Brown, P. Matousek, M. Towrie, E. T. J. Nibbering, P. Foggi, F. V. R. Neuwahl J. Phys. Chem. A. 2001, 105, 3709.

[235] A. N. Bader, F. Ariese, C. Gooijer J. Phys. Chem. A. 2002, 106, 2844.

[236] E. de Rijke, H. C. Joshi, H. R. Sanderse, F. Ariese, U. A. T. Brinkman, C. Gooijer Anal. Chim. Acta. 2002, 468, 3.

[237] C. L. Dunford, G. J. Smith, E. E. Swinny, K. R. Markham Photoch. Photobio. Sci. 2003, 2, 611 .

[238] P. T. Chou, Y. C. Chen, W. S. Yu, Y. M. Cheng Chem. Phys. Lett. 2001, 340, 89.

[239] M. Sarkar, P. K. Sengupta J. Photochem. Photobiol. A. 1989, 48, 175.

[240] R. Schipfer, O. S. Wolfbeis, A. Knierzinger J. Chem. Soc., Perkin Trans. 2. 1981, 1443.

[241] S. Prasad De, S. Ash, H. Bar, D. Kumar Bhui, S. Dalai, A. Misra J. Mol. Struct.: THEOCHEM. 2007, 824, 8.

[242] C. M. Marian Spectrochim. Acta Part A. 2009, 73, 1.

[243] S. M. Beyhan, A. W. Gotz, F. Ariese, L. Visscher, C. Gooijer J. Phys. Chem. A. 2011, 115, 1493.

[244] W. L. Jorgensen, D. S. Maxwell, J. Tirado-Rives J. Am. Chem. Soc. 1996, 118, 11225 .

[245] H. J. Bohm, I. R. Mcdonald, P. A. Madden Mol. Phys. 1983, 49, 347.

[246] N. Ferré, J. G. Ángyán Chem. Phys. Lett. 2002, 356, 331.

[247] J. R. Platt J. Chem. Phys. 1949, 17, 484-495.

[248] T. Gustavsson, N. Sarkar, E. Lazzarotto, D. Markovitsi, V. Barone, R. Improta J. Phys. Chem. B. 2006, 110, 12843.

[249] F. Santoro, V. Barone, T. Gustavsson, R. Improta J. Am. Chem. Soc. 2006, 128, 16312. 